FINAL REPORT

U.S. Department of Energy

\title{
In-Situ Characterizations of Dense Non-Aqueous Phase Liquids Using Partitioning Tracers
}

\author{
Gary A. Pope \\ Daene C. McKinney \\ The University of Texas at Austin \\ Austin, TX \\ Akhil Datta Gupta \\ Texas A\&M University \\ College Station, TX \\ Richard E. Jackson and Minquan Jin \\ Duke Engineering Services, Inc. \\ Austin, TX \\ Research Associates \\ Mojdeh Delshad, Bruce Rouse, Prasad Saripalli \\ Graduate Research Assistants \\ Indranil Barman, Varadarajan Dwarakanath, Neil Deeds, \\ Jeff Edgar, Jean-Philippe Nicot, G. Allen Whitley, Jr.
}

Project Number: 55216

Grant Number: DE-FG07-96ER14720

Nicholas Woodward, Project Officer

U. S. Department of Energy

Office Code ER-15

Washington, DC 20585
Gordon Roesler, Project Officer

U. S. Department of Energy

Office Code EM-52

Washington, DC 20585

\section{Carol Van Lente, Contract Administrator \\ U. S. Department of Energy Idaho Operations Office 850 Energy Drive MS 1221 \\ Idaho Falls, Idaho 83401-1563}

Prepared by

The University of Texas at Austin

Center for Petroleum and Geosystems Engineering

Austin, TX 78712

Project Duration: September 15, 1996 to September 14, 1999 


\section{Table of Contents}

Section 1: Executive Summary ……………………....................................................

Section 2: Inverse Modeling of Partitioning Interwell Tracer Tests: A Streamline Approach................................................................................................ 5

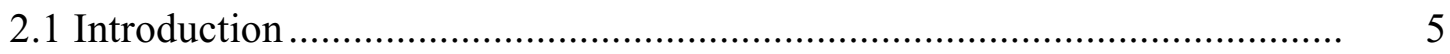

2.2 Streamline-Based Inversion .................................................................. $\quad 7$

2.3 Applications .......................................................................................... 14

2.4. Summary and Conclusions.................................................................... 19

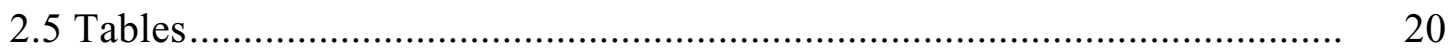

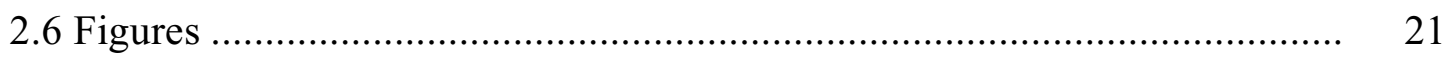

Section 3: Tracer Mass Transfer in Porous Media …………………………………......... 33

3.1 Two-resistance Theory ……………………………………………..... 33

3.2 Estimation of Phase Mass Transfer Resistances .......................................... 35

3.3 Relative Importance of NAPL and Water Phase Resistances......................... 40

3.4 One Dimensional Transport Model with Mass Transfer ................................. 42

3.5 Implementation of Tracer Mass Transfer in UTCHEM ................................. 51

3.6 UTCHEM Simulation of Field PITT ......................................................... 54

3.7 Summary and Conclusions...................................................................... 56

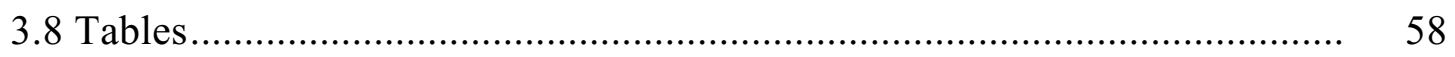

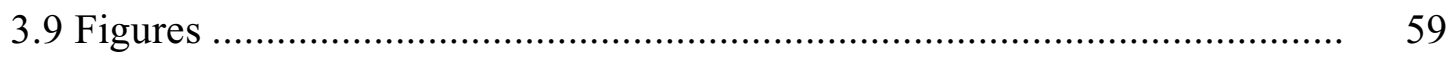

Section 4: Partitioning Tracer Transport in Fractured Media ………………………......... 75

4.1 Partitioning Tracers in Fractured Media with Mass Transfer ......................... 75

4.2 Sensitivity Analysis ............................................................................. 81

4.3 Combined Impacts of Matrix Diffusion and Water/NAPL Mass

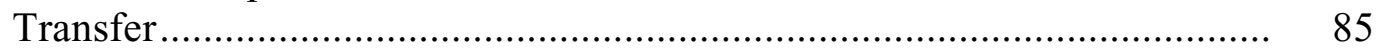

4.4 Summary and Conclusions.................................................................... 87

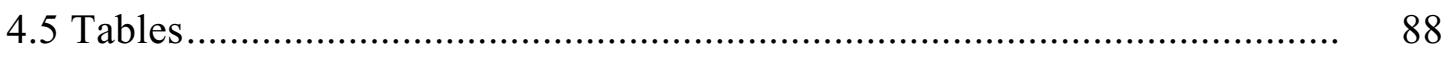




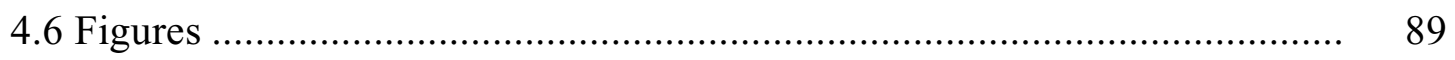

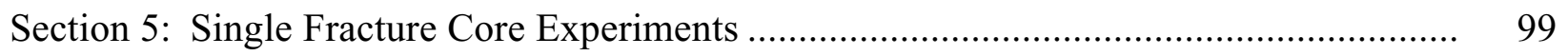

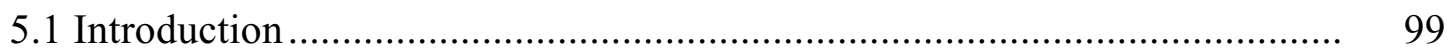

5.2 Experimental Materials......................................................................... 99

5.3 Experimental Methods ....................................................................... 101

5.4 Modeling the Output Curves...................................................................... 107

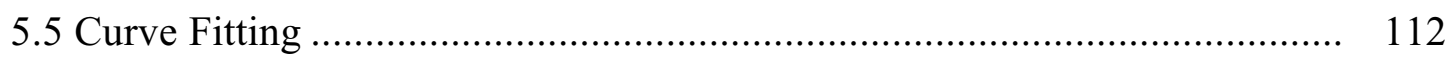

5.6 Results for Single Fracture Experiments, No NAPL.................................... 115

5.7 Results for Single Fracture Experiments With NAPL.................................. 123

5.8 Summary and Conclusions...................................................................... 127

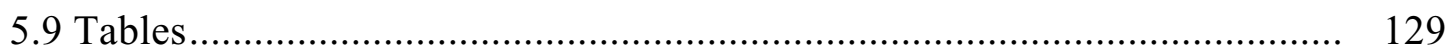

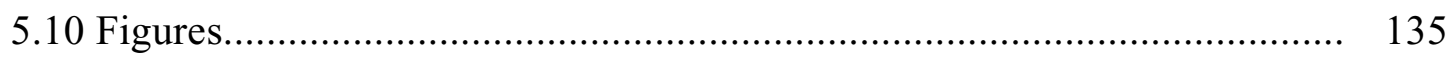

Section 6: Three-Dimensional Transport Simulations...................................................... 157

6.1 The Dual Porosity Model............................................................................ 157

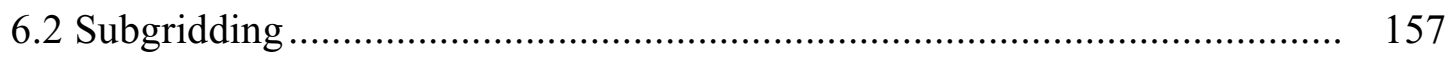

6.3 Modeling a DNAPL Spill ...................................................................... 161

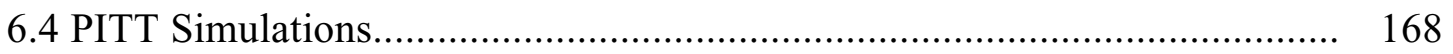

6.5 Summary and Conclusions........................................................................ 174

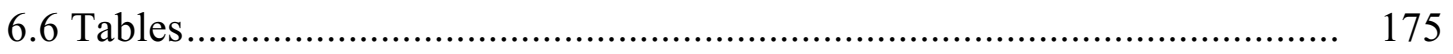

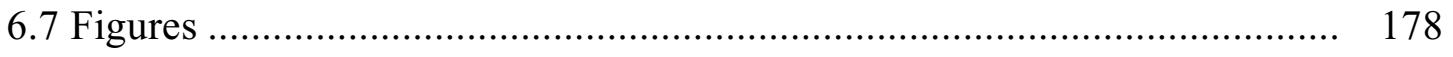

Section 7: Summary, Conclusions and Recommendations for Future Work......................... 205

7.1 Summary and Conclusions....................................................................... 205

7.2 PITT Design in Fractured Media................................................................... 209

7.3 Recommendations for Future Work ……………….................................... 210

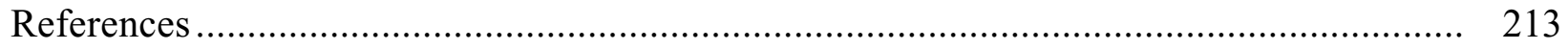




\section{Section 1: Executive Summary}

Major advances have been made during the past three years in our research on interwell partitioning tracers tests (PITTs). These advances include (1) progress on the inverse problem of how to estimate the three-dimensional distribution of NAPL in aquifers from the tracer data, (2) the first ever partitioning tracer experiments in dual porosity media, (3) the first modeling of partitioning tracers in dual porosity media (4) experiments with complex NAPLs such as coal tar,

(5) the development of an accurate and simple method to predict partition coefficients using the equivalent alkane carbon number approach, (6) partitioning tracer experiments in large model aquifers with permeability layers, (7) the first ever analysis of partitioning tracer data to estimate the change in composition of a NAPL before and after remediation (8) the first ever analysis of partitioning tracer data after a field demonstration of surfactant foam to remediate NAPL and (9) experiments at elevated temperatures. Highlights of some of these research accomplishments are briefly summarized below.

We have developed a new analytic approach that has several advantages over existing approaches for inversion of tracer data. First, the technique utilizes an extremely efficient threedimensional multiphase streamline simulator as a forward model. Second, the parameter sensitivities are formulated in terms of one-dimensional integrals of analytic functions along the streamlines. Thus, the computation of sensitivities for all model parameters requires only a single simulation run to construct the velocity field and generate the streamlines. The inversion of tracer data is then performed using a two-step iterative linearization that involves first lining-up the breakthrough times at the extraction wells and then matching the production history. Our approach follows from an analogy between streamlines and ray tracing in seismology. The 
inverse method is analogous to seismic waveform inversion and thus, allows us to utilize efficient methods from geophysical imaging.

In estimation of spatially distributed parameters such as permeability and DNAPL saturation, it has been recognized for a long time that data is never abundant enough to constraint the parameters uniquely. The new approach taken in this research is to recognize this fact from the beginning and to solve for an ensemble of solutions, each equally valid. The nonuniqueness issue is attacked from different perspectives. First, Genetic Algorithms (GAs), an efficient optimization search method, are used. GAs span the whole search space, act as a directed Monte-Carlo search and produces the initial set of valid solutions. Each solution is then refined using a classical gradient-based method. Second, the solutions are forced to be smooth in some sense by adding a penalty term to the objective function. Solutions showing unnatural variations of parameter values are thus penalized. Third, a cluster analysis is used to group together similar solutions. Characterization of the uncertainty of the solution, often overlooked, is treated fully through two approaches. They both involve the computation of the sensitivity matrix but extract different information from it. This approach is currently being tested on both realistic synthetic examples and field data from the saturated PITTs at Hill AFB and the unsaturated PITTs at Kirtland AFB.

The first ever partitioning tracer experiments were completed in dual-porosity rock. These studies are needed to understand the behavior of partitioning tracers in such media, to compare with flow and transport models, to select the best tracers and operating conditions, and ultimately to determine the viability and applicability of partitioning tracer technology for characterizing nonaqueous phase liquid contamination in dual-porosity aquifers. The first experiment was done in a Berea sandstone with a single fracture with and without NAPL in the 
fracture. The results of these experiments clearly show the combined impacts of partitioning to the NAPL, fracture transport and matrix diffusion. To the best of our knowledge, no such experiments have been reported in the literature, so the experimental methodology, partitioning tracer technology and interpretation approach are all entirely new and must continue to be developed. A variety of other tracers with different diffusion coefficients and partition coefficients have been tested to start this process. An analytical model was used to interpret these partitioning tracer single-fracture studies. The model shows very good agreement with the laboratory results. The agreement indicates that the physical processes assumed in the model are similar to the actual processes occurring in the laboratory experiments. The understanding of these processes that we have gained is essential to continued development of partitioning tracer technology as applied to fractured aquifers. The first ever field-scale modeling is under way using UTCHEM, a simulator which includes the necessary features for modeling both tracer partitioning and dual-porosity tracer transport. These simulations are essential for our understanding of the relative importance of various parameters and characteristics for partitioning tracer test design in fractured media. Inspectional analysis has been used to determine the appropriate nondimensional scaling groups to aid in the understanding and generalization of our studies with fractured media.

Two vadose zone partitioning interwell tracer tests were completed at Kirtland Air Force Base to evaluate a radio frequency enhanced soil vapor extraction remediation technology. The remediation effort was a project of the Advanced Applied Technology Demonstration Facility. This effort was the first attempt to estimate the change in the composition of the NAPL after remediation. The Peng-Robinson equation-of-state was used to model the thermodynamic interaction between the hydrocarbons and the partitioning tracers. From this interaction, the 
compositional effects were determined. This is the first time that both the volume of NAPL removed from the soil and the change in composition of the remaining NAPL due to the remedial action have been simultaneously measured in a field demonstration. This work is relevant to the many field sites where the NAPL consists of a complex chemical mixture and where the remediation process causes large changes in its composition such as when the NAPL is heated as part of the remedial process.

Alcohol tracer partition coefficients for coal tar were measured and compared to similar values for several different NAPLs. Coal tar is a multicomponent dense, nonaqueous phase liquid (DNAPL) primarily composed of polycyclic aromatic hydrocarbons. Despite the great complexity of coal tar, the results show that the partition coefficients can be predicted using the equivalent alkane carbon number approach just as well as for simple NAPLs such as trichloroethylene and decane. We have also investigated other very complex NAPLs such as a fuel oil with very high viscosity and in all cases we have found that the equivalent alkane carbon number approach is useful for correlating the tracer partition coefficients. 


\section{Section 2: Inverse Modeling of Partitioning Interwell Tracer Tests: A Streamline Approach}

\subsection{Introduction}

It is now well recognized the presence of NAPLs poses a significant impediment to aquifer restoration. In order for any remediation technique to be successful, it is essential that the NAPL distribution be properly characterized. Partitioning tracer test is a promising technique for characterizing NAPL distribution in the subsurface because of accessibility to large volumes of the contaminant. During partitioning interwell tracer tests a suite of tracers with a range of NAPL-water partitioning coefficients are injected into the subsurface and are recovered down gradient at the extraction wells. A conservative or non-partitioning tracer is also injected during the test. Because of the presence of NAPL, partitioning tracers are retarded compared to the nonpartitioning tracer. The chromatographic separation between these tracers can be utilized to infer spatial distribution of NAPL in the subsurface (Jin et al., 1995). When the tracer is sampled at multiple vertical and areal locations, then the tracer tests can be used to estimate the 3-D distribution of NAPL in the subsurface (Annable et al., 1996). Such information is of obvious importance in the design and implementation of appropriate remediation schemes.

Previous efforts towards estimating NAPL saturation using partitioning interwell tracer tests have mostly utilized the method of moments (Jin et al., 1995; Wilson and Mackay, 1997; Annable, 1998). Such approaches are well-suited to estimate the average residual NAPL saturation in the tracer swept region but cannot determine the spatial distribution of NAPL saturation. James et al. (1997) introduced a stochastic approach that utilizes a cokriging-based algorithm to estimate spatial distribution of temporal moments for both conservative and 
partitioning tracers. The approach was validated using a synthetic example. The method utilizes first order approximations to the moment equations that might limit it to small variations in subsurface properties. Further complications may arise from the requirement of prior estimates for various covariance and cross-variance functions that may be difficult to obtain in field situations.

In recent years, inverse problems that utilize solute concentration response have received increased attention in the literature (Harvey and Gorelick, 1996; Hyndman and Gorelick; Medina and Carrera, 1996). Computational efforts associated with such inverse modeling still remains a significant factor that deters the routine use of concentration data (Anderson and Hill, 1999). The formulation of the inverse problems typically requires the computation of sensitivities of concentration to changes in model parameters. That is, we must compute the change in concentration response resulting from a small perturbation in subsurface properties such as permeability, porosity or fluid saturation. The computation of such sensitivities can be classified into three broad categories: perturbation approaches, direct algorithms and the adjoint state methods. The relative merits of these methods have been discussed in the literature (Yeh; 1986; McLaughlin and Townley, 1996).

Recent advances in streamline simulation techniques make it possible to incorporate time variant measurements efficiently for estimating subsurface parameters within the framework of inverse modeling. (Vasco and Datta-Gupta, 1999; Datta-Gupta et al., 1998). Streamline models can be advantageous in two ways. First, the streamline simulator can serve as an efficient forward model for the inverse problem (Datta-Gupta and King, 1995; Crane and Blunt, 1999). Second, and more importantly, parameter sensitivities can be formulated as one dimensional integrals of analytic function along streamlines. The computation of sensitivity for all model 
parameters then requires a single simulation run. The sensitivity computations can exploit the analogy between streamlines and seismic ray tracing based on the observation that the streamline transport equations can be cast in the form of the Eikonal equation, the governing equation for travel time tomography (Vasco et al., 1999). This allows us to use efficient techniques from geophysical inverse theory to match both conservative and partitioning tracer responses. Inversion of tracer response can be carried out in a manner analogous to seismic waveform inversion (Zhou, et al., 1995). This involves matching the breakthrough or 'first arrival' of the tracer response followed by matching of 'amplitudes' of the tracer response, for example the peak height and peak location. Such two-step procedure makes the solution less sensitive to the choice of initial model. An additional feature of the method is that it prevents the solution from being trapped into secondary peaks particularly for field applications whereby the tracer response is frequently characterized by multiple peaks.

\subsection{Streamline-Based Inversion}

In this section, we discuss the mathematical formulation for the analysis and inversion of partitioning interwell tracer tests using the streamline approach. The principal components are forward modeling of the tracer response, computation of sensitivities of tracer response to subsurface properties and finally, history matching or data inversion.

\subsubsection{Modeling of Tracer Transport}

Forward model relates unknown parameters, such as hydraulic conductivity and NAPL saturation to tracer response data. Since tracers are often injected as a finite slug in small quantities, avoiding numerical dispersion in tracer transport modeling is a major concern. Computational burden associated with repeated forward calculations is another aspect to 
consider. To ensure accuracy and efficiency of computation, we have used a three-dimensional multiphase streamline model for flow and transport calculations (Datta-Gupta and King, 1995; Batycky et al., 1997).

The details of streamline simulation can be found elsewhere (King and Datta-Gupta, 1998; Crane and Blunt, 1999). Briefly, in this approach we decouple flow and transport by a coordinate transformation from the physical space to one following flow directions viz. the tracer time of flight along streamlines. The time of flight is defined as:

$$
\tau=\underset{\psi}{s(\mathbf{x}) d r}
$$

where we have introduced a slowness as follows:

$$
s(\mathbf{x})=\frac{1}{|\mathbf{v}(\mathbf{x})|}=\frac{n(\mathbf{x})}{K(\mathbf{x})|\phi(\mathbf{x})|}
$$

where $v$ denotes interstitial velocity, $n$ is the porosity, $K$ is the hydraulic conductivity, and $\phi$ is the piezometric head or pressure.

Consider the convective transport of a neutral tracer. The governing equation is given by

$$
\frac{f C(\mathbf{x}, t)}{f t}+\mathbf{v} ? \quad C(\mathbf{x}, t)=0
$$

where $C$ represents the tracer concentration. The streamline approach relies on a coordinate transformation from the physical space to the time-of-flight coordinates using the operator identity (King and Datta-Gupta, 1998)

$$
v ?=\frac{f}{f \tau} .
$$

The coordinate transformation reduces the multidimensional transport equation into a series of one dimensional equations along streamlines, 


$$
\frac{f C(\tau, t)}{f t}+\frac{f C(\tau, t)}{f \tau}=0 .
$$

The tracer response at a producing well can be obtained by simply integrating the contributions of individual streamlines (Datta-Gupta and King, 1995)

$$
C(t)={ }_{\text {all } \psi} C_{0} \bar{t}-s(\mathbf{x}) d r \sqrt[v]{d} d \psi
$$

where $C_{0}$ is tracer concentration at injection well. If we include longitudinal dispersion along streamlines, then the tracer concentration at the producing well will be given by (Gelhar and Collins, 1971)

$$
C(t)={ }_{\text {all } \psi} \frac{\exp \left[-(t-\tau(\psi))^{2} /(4 \alpha \omega)\right]}{\sqrt{4 \alpha \omega}} d \psi
$$

where $\alpha$ is longitudinal dispersivity and $\omega=\frac{d r}{\psi^{v^{2}}}$.

During partitioning interwell tracer tests the retardation of partitioning tracers in the presence of NAPL saturation can simply be expressed as increased travel time along streamlines. This in turn results in an increased slowness as follows:

$$
s(\mathbf{x})=\frac{1}{|\mathbf{v}(\mathbf{x})|}\left(S_{w}+K_{N} S_{N}\right)=\frac{n(\mathbf{x})}{K(\mathbf{x})|\phi(\mathbf{x})|}\left(S_{w}+K_{N} S_{N}\right)
$$

where $S_{w}$ and $S_{N}$ denote water and NAPL saturation and $K_{N}$ is the partitioning coefficient of tracer defined as the ratio of tracer concentration in oil phase to that in water phase.

\subsubsection{Analytic Sensitivity Computations}

Sensitivity calculations constitute a critical aspect of inverse modeling. By sensitivity, we mean the partial derivative of the tracer response with respect to model parameters such as hydraulic 
conductivity, porosity and NAPL saturation. Although several methods are available for computing sensitivities, for example, numerical perturbation method, sensitivity equation method, or adjoint state method (Yeh, 1986; Sun and Yeh, 1990), these are limited by their computational costs and complex implementations. The streamline approach provides an extremely efficient means for computing parameter sensitivities using a single forward simulation. The sensitivities can be computed analytically and only require evaluation of onedimensional integrals along streamlines.

Consider a small perturbation in subsurface property about an initial model. The corresponding changes in slowness and tracer concentration can be written as:

$$
\begin{aligned}
s(\mathbf{x}) & =s^{0}(\mathbf{x})+\delta s(\mathbf{x}) \\
C(\mathbf{x}, t) & =C^{0}(\mathbf{x}, t)+\delta C(\mathbf{x}, t)
\end{aligned}
$$

where $\mathrm{s}^{0}$ and $C^{0}$ are reference slowness and tracer concentration, respectively.

If we assume that streamlines do not shift as a result of these small perturbations, then we can relate changes in tracer time of flight and concentration to changes in slowness (Vasco et al., 1999):

$$
\begin{gathered}
\delta \tau(\mathbf{x})={ }_{\psi} \delta s(\mathbf{x}) d r \\
\delta C(\mathbf{x}, t)=-\mathcal{C}_{0}^{\otimes_{0}}\left(t-s_{\psi}^{0} d r\right) \quad \delta s(\mathbf{x}) d r
\end{gathered}
$$

Because slowness is a composite response, its variation will be given by

$$
\delta s(\mathbf{x})=\frac{f_{s}(\mathbf{x})}{f K} \delta K(\mathbf{x})+\frac{f_{s}(\mathbf{x})}{f n} \delta n(\mathbf{x})+\frac{f_{s}(\mathbf{x})}{f S_{w}} \delta S_{w}
$$

where the partial derivatives are as follows 


$$
\begin{aligned}
& \frac{f s(\mathbf{x})}{f K}=\frac{-n(\mathbf{x})}{K^{2}(\mathbf{x})|\phi|}\left(S_{w}+K_{N} S_{N}\right)=\frac{-s(\mathbf{x})}{K(\mathbf{x})}\left(S_{w}+K_{N} S_{N}\right) \\
& \frac{f s(\mathbf{x})}{f n}=\frac{1}{K(\mathbf{x})|\phi|}\left(S_{w}+K_{N} S_{N}\right)=\frac{s(\mathbf{x})}{n(\mathbf{x})}\left(S_{w}+K_{N} S_{N}\right) \\
& \frac{f s(\mathbf{x})}{f S_{w}}=\frac{n(\mathbf{x})}{K(\mathbf{x})|\phi|}\left(1-K_{N}\right)=s(\mathbf{x})\left(1-K_{N}\right)
\end{aligned}
$$

Note that in the above expressions, we have ignored pressure changes resulting from small variations in subsurface parameters. Also, for unit partitioning coefficient, the tracer response will be insensitive to saturation changes as one might expect. Tracer travel time and concentration sensitivities with respect to hydraulic conductivity, porosity, and water saturation can be obtained by integrating (2.10) over all streamlines contributing to a producer.

The expressions in (2.12) only involve quantities that are readily available once we generate the velocity field and define the streamline trajectories in a streamline simulator. Thus, in a single forward run of streamline simulation we derive all the sensitivity coefficients required to solve the inverse problem. Figure 2.1 shows tracer concentration sensitivity to hydraulic conductivity, porosity and water saturation in a quarter five-spot computed using the streamlinebased analytic method. For comparison, we have also shown results using a numerical perturbation method whereby each parameter is perturbed at a time and the tracer response is recomputed. Excellent agreement in the results indicates the validity of our analytic approach.

\subsubsection{Estimating Subsurface Properties: Inverse Modeling}

During inverse modeling, we want to minimize the differences between observed tracer responses and model predictions to estimate unknown parameter. Mathematically, this can be expressed as

$$
\min _{\mathbf{m}}\|\mathbf{d}-\mathbf{g}[\mathbf{m}]\|^{2}
$$


where $\mathbf{d}$ is the data vector with $N$ observations, $\mathbf{g}$ is the forward model, $\mathbf{m}$ is the vector of $M$ parameters, and $\|\cdot\|$ denotes the Euclidean norm. Because of non-linearity between data and model parameters, we must resort to an iterative procedure for the minimization. For example, at the $k$-th iteration step we obtain the following using a first order Taylor series expansion of $g[\mathbf{n}]$ around $\mathbf{m}^{k}$,

$$
\mathbf{g}[\mathbf{m}]=\mathbf{g}\left[\mathbf{m}^{k}\right]+\mathbf{G}^{k} \delta \mathbf{m}
$$

where $\delta \mathbf{m}$ is the parameter perturbation at $k$-th step and Gis the sensitivity matrix with sensitivity coefficient entries as before. For example, $G_{i j}$ denotes the sensitivity of the $i$-th data point with respect to the $j$-th parameter and will be given by:

$$
G_{i j}=\frac{f d_{i}}{f m_{j}} .
$$

In general, we will have many observations from several different wells. The differences between the observed and calculated tracer response at the $k$-th iteration step will result in a data misfit vector given by

$$
=\mathbf{d}-\mathbf{g}\left[\mathbf{m}^{k}\right] .
$$

The data misfit vector can be related to the changes in the model parameter estimates through the following system of equations

$$
=\mathbf{G}^{k} \delta \mathbf{m} \text {. }
$$

Our approach follows an iterative minimization procedure by solving (2.17) at each step or equivalently minimizing the linear least squares

$$
\|-\mathbf{G} \delta \mathbf{m}\|^{2}={ }_{i=1}^{N-\varepsilon_{i}}-{ }_{j=1}^{M} G_{i j} \delta m_{j} \underset{\jmath}{\sqrt{ }}
$$

and updating our parameter vector at each step during iteration 


$$
\mathbf{m}=\mathbf{m}^{k}+\delta \mathbf{m} .
$$

In field situations, very often we have a large number of unknown parameters and limited measurements. Thus, the inverse problem tends to be ill-posed. Such ill-posed problems can suffer from non-uniqueness and instability in solution. To circumvent these problems, we augment the linear system of equation (2.18) by incorporating additional penalty terms, called regularization (Constable et al., 1987; Borchers et al., 1997; Liu and Ball, 1999). Two common approaches are to include a model norm constraint and a model roughness constraint. The norm constraint ensures that our final model is not significantly different from our initial model. This makes physical sense because typically our initial model already incorporates sufficient geologic and other prior information. The roughness constraint accounts for the fact that tracer data is an integrated response and is best suited to resolve large-scale trends rather than small-scale fluctuations. The penalized objective function to be minimized is now given by

$$
\|-\mathbf{G} \delta \mathbf{m}\|^{2}+\beta_{1}^{2}\|\delta \mathbf{m}\|^{2}+\beta_{2}^{2}\|\mathbf{L} \delta \mathbf{m}\|^{2}
$$

The minimization of (2.20) is equivalent to solving the following augmented linear system in a least square sense,

$$
\min _{\delta \mathbf{m}}\left\|\begin{array}{cr}
\mathbf{G} & \\
\beta_{1} \mathbf{I} & \delta \mathbf{m}- \\
\beta_{2} \mathbf{L} & \mathbf{0}
\end{array}\right\|^{2}
$$

where $\beta \mathrm{s}$ are the weighting factors for the model norm and roughness penalties, $\mathbf{I}$ is an identity matrix and $\mathbf{L}$ is a spatial difference operator, typically the second spatial derivative of parameters measuring the model roughness. An iterative sparse matrix solver, LSQR (Paige and Saunders, 1982; Golub and Van Loan, 1989) is used for solving this augmented linear system efficiently. 


\subsubsection{Two-step Procedure}

Our approach to determination of NAPL saturation based on partitioning tracer response consists of two steps. First we invert the conservative tracer response to infer spatial distribution of hydraulic conductivity in the subsurface. Next, utilizing this hydraulic conductivity distribution, we invert the partitioning tracer response to determine the spatial distribution of residual NAPL. The underlying assumption here is that the conservative tracer response is primarily governed by the hydraulic conductivity variations whereas the partitioning tracer response is influence by both hydraulic conductivity and NAPL distribution. Numerical experiments indicate that this is a reasonable assumption for NAPL saturation below 15\% (Yoon, 1999). The streamline-based approach proposed here facilitates the two-step procedure because we can compute sensitivities of tracer response with respect to hydraulic conductivity as well as saturation in a single simulation run.

\subsection{Applications}

In this section, we first consider a synthetic example and then a field application of our proposed approach. The synthetic example illustrates our procedure for analysis of partitioning tracer tests to characterize NAPL saturation in the subsurface. The field example is from the Hill Airforce base, Utah and demonstrates the feasibility of the approach for analyzing partitioning tracer response in field situations.

\subsubsection{A Synthetic Example: Partitioning Test in a Nine-Spot Pattern}

The well configuration for this example is a 9-spot pattern with a central injector and 8 surrounding producers. The spatial distribution of NAPL saturation, shown in Fig. 2.2, is generated using a stochastic moving average method. A uniform hydraulic conductivity field is 
assumed for this example. Tracers are injected in the central well and sampled at the producers. For the synthetic case we consider the hydraulic conductivity field to be known and use the partitioning tracer responses only for determining saturation distribution. The partitioning tracer responses are shown in Fig. 2.3. For comparison purposes, we have also superimposed the tracer responses from our initial model that assumes a constant NAPL saturation of $15 \%$.

Our inversion proceeds in two stages in a manner analogous to seismic waveform fitting (Zhou et al., 1995). We first match the arrival times of the peak concentrations at the producing wells. Only then, after the peaks have been 'lined up', are the histories themselves matched. Our experience has shown that it also makes the solution relatively insensitive to our selection of the prior model. During arrival time matching, we solve a system of equations equivalent to (2.17) relating the peak arrival times $\mathbf{T}$ at the wells to subsurface property variations

$$
\delta \mathbf{T}=\mathbf{G} \delta \mathbf{m}
$$

with the elements of the sensitivity matrix $\mathbf{G}$ are given by (2.10a).

Figure 2.4 shows the results after matching the peak arrival times. Clearly, all the peak times are now in close agreement although there are still some discrepancies in the amplitudes of the tracer response. Figure 2.5 shows the NAPL saturation distribution after matching the peak arrival times. It is apparent that most of the major trends in the saturation distribution are already resolved at this stage (Vasco et al., 1999). The computation time for the arrival time match was less than 10 seconds on a Pentium III PC. The arrival time inversion is followed by the amplitude inversion whereby we match the complete tracer history. Figures 2.6 and 2.7 are the final matches to the tracer response and the NAPL saturation distribution, respectively. The improvement over the arrival time inversion is rather minimal although the results now seem to include smaller-scale variations in saturation. The total computation time for this example was 
29 seconds. The incremental benefit of matching the amplitudes of the tracer response is not very significant considering that most of the computation time is devoted to this stage.

\subsubsection{Inversion of Hill Air Force Base Interwell Tracer Tests}

The details of the partitioning tracer test conducted at the Hill Airforce base can be found in Annable et al. [1994, 1998]. The aquifer consists of sand, gravels (with some large cobbles), and clays with a mean permeability of 20 darcies. The base of the aquifer is defined by an impermeable clay layer. The NAPL, which is lighter than water, is in the form of a plume covering several acres.

An isolation test cell was installed for the purpose of evaluating the use of cosolvents as a remediation tool. The cell consisting of a sealable sheet pile barrier system measures 3.5 by $4.3 \mathrm{~m}$ and extends to a depth of $9.1 \mathrm{~m}$ below the ground surface, some $3 \mathrm{~m}$ below the confining unit of the aquifer. Multiple tracers were injected using four injection wells at one end of the cell. Tracer responses were measured at three extraction wells at the opposite end and 12 multilevel samplers between the injection and extraction wells as depicted in Fig. 2.8. For our analysis, we have used Bromide as the conservative tracer and 2,2-Dimethyl-3-Pentanol (DMP) as the partitioning tracer from the suites of field tracer response. We chose DMP as the partitioning tracer because of its higher partitioning coefficient compared to other tracers as shown in Table 2.1.

We model the lower portion of the test cell using $14 \times 11 \times 10$ grid blocks with dimensions of approximately $0.3 \mathrm{~m}$ horizontally and $0.15 \mathrm{~m}$ vertically. The choice of the grid was largely dictated by the spacing of multilevel samplers to capture spatial variations between samplers both laterally and vertically. To start with, we assume a uniform distribution of hydraulic conductivity within the test cell. Tracer responses from the initial model and observed tracer responses at five selected sampling locations are shown in Fig. 2.9. First, conservative tracer 
responses measured at each sampling location are matched to infer hydraulic conductivity distribution within the test cell. Figure 2.10 shows the result after matching the peak arrival times at the same five locations. In general, matching peak arrival times also result in a substantial improvement in the overall tracer history match as can be seen here. The observed and calculated peak arrival times at all sampling locations are shown in Fig. 2.11. The computation time for the arrival time match was just 37 seconds on the Pentium III. The estimated hydraulic conductivity field is shown in Fig. 2.12.

Next we invert the partitioning tracer response starting with the hydraulic conductivity field derived from the conservative tracer response and a uniform initial NAPL saturation distribution. Figure 2.13 compares the observed and calculated partitioning tracer response at five selected locations based on the initial model. Figure 2.14 shows the results after inversion. The observed and calculated peak arrival times at all sampling locations after 14 iterations are shown in Fig. 2.15. The final NAPL distribution is shown in Fig. 2.16. The total computation time for this example was 1 minutes and 34 seconds on the Pentium III. Our results indicate an average NAPL saturation of about $6 \%$ with higher saturation towards the lower part of the test cell. The average NAPL saturation is in agreement with previous estimates using the moment analysis (Annable et al., 1998). The NAPL distribution is consistent with soil core analysis indicating higher NAPL saturation clustered towards the lower portion of the cell (Annable et al., 1994). For comparison purposes, we also calculated tracer retardation factors defined as the ratio of average travel times for the partitioning tracer and the conservative tracer at sampling locations (Jin et al., 1995; Annable et al., 1998). A qualitative comparison of our inversion result with the spatial distribution of retardation factors (Fig. 2.17) seems to further validate our results. Streamline distribution for the final model is shown in Fig. 2.18. 
The streamline approach allows us to readily calculate the volumetric sweep from the distribution of tracer travel times in the test cell as shown in Fig. 2.19. The contours represent tracer swept volumes at different times. The tracer swept volume can be calculated using the following expression

$$
V_{S}(t)=\underset{\text { all } \psi}{d \psi} d \tau(\psi) \theta(t-\tau) q(\psi)
$$

where $\theta$ is the Heaviside function and $q$ is volumetric flow rate assigned to each streamline. Figure 2.20 shows the volumetric sweep efficiency as a function of injection time where the volumetric sweep efficiency is defined by the ratio of tracer swept volume to total bulk volume. For all practical purposes, the test cell is completely swept after an injection period of five days.

A final step in inverse modeling or parameter estimation is an assessment of the solution to quantify the uncertainty associated with the parameter estimates. For this we resort to resolution analysis (Menke, 1989; Datta-Gupta et al., 1997). Resolution of estimates varies between 0 and 1 with 0 being completely undetermined and 1 being completely determined. Figure 2.21 shows the resolution of saturation estimates using the partitioning tracer data. Higher resolution is observed towards the bottom portion of the cell with high NAPL saturations. Also, high resolution is observed along flow paths between injectors and producers where sensitivities are high. Similar trends were also observed by James et al. (1997) using a stochastic inversion approach.

One of the major advantages of the streamline approach presented here is its computational efficiency because of the analytic computation of parameter sensitivities. For benchmarking purposes, we compared our approach with a gradient free global optimization technique, simulated annealing. In simulated annealing, parameter values are perturbed at random by drawing from a pre-defined probability distribution for each parameter. The method 
provides a mechanism of probabilistic hill climbing that allows the solution to escape from local minimum. Figure 2.22 shows the NAPL saturation distribution using the simulated annealing approach. Overall, the NAPL distribution follows similar trends as in Fig. 2.16. However, the computation time required was 15 hours for conservative tracer inversion followed by 16 hours for partitioning tracer inversion. This is an increase of more than 3 orders of magnitude compared to our proposed approach. Table 2.2 summarizes the computation times.

\subsection{Summary and Conclusions}

We have described a streamline-based inversion technique to estimate NAPL saturation using partitioning interwell tracer tests. Primary advantage of our proposed method is its computational efficiency, three orders of magnitude faster than simulated annealing for the example presented here. This makes our method particularly attractive for analysis of large-scale field tests. The speed of computation can be attributed mainly to our analytical sensitivity calculations using the streamline approach. Our inversion scheme is analogous to seismic waveform inversion whereby we first match the arrival times followed by amplitudes of the tracer response. This makes the inversion method robust, relatively insensitive to our initial model and also prevents the solution from being trapped in secondary tracer peaks. This is particularly important for field application because field tracer tests are very often characterized by multi-peaked response. Following specific conclusions can be drawn based on our results:

1. We have proposed a new analytical approach for computing sensitivities of tracer response to subsurface parameters such as porosity, hydraulic conductivity and NAPL saturation. The approach utilizes a streamline simulator to model tracer transport and requires a single simulation run to compute sensitivities with respect to all relevant parameters. 
2. A two-step inversion approach to analysis of partitioning tracer tests has been proposed. Our method is extremely computationally efficient and outperforms simulated annealing by three orders of magnitude.

3. Inversion of tracer data by analogy to seismic waveform inversion, that is matching arrival time first followed by matching amplitudes, has resulted in a robust inversion scheme.

4. The power of our method has been illustrated using synthetic and field examples.

\subsection{Tables}

Table 2.1: Tracer partitioning coefficients for Hill AFB case.(Annable et al., 1994)

\begin{tabular}{|c|c|}
\hline Tracer & $\mathbf{K}_{\mathbf{0}}$ \\
\hline Bromide & 0.0 \\
\hline Ethanol & 0.1 \\
\hline n-Pentanol & 1.4 \\
\hline n-Hexanol & 4.6 \\
\hline 2,2-Dimethyl-3-Pentanol & 12.9 \\
\hline
\end{tabular}

Table 2.2: Performance comparison between the proposed inversion method and the simulated annealing method in terms of computation CPU time on Pentium III PC.

\begin{tabular}{|r|c|c|c|}
\hline Inversion Step & $\begin{array}{c}\text { Proposed Inversion } \\
\text { Method }\end{array}$ & $\begin{array}{c}\text { Simulated } \\
\text { Annealing }\end{array}$ & $\begin{array}{c}\text { Speed-up } \\
\text { Factor }\end{array}$ \\
\hline Conservative Tracer Inversion & $00: 00: 37$ & $15: 07: 18$ & 1,471 \\
\hline Partitioning Tracer Inversion $^{\mid} 00: 00: 57$ & $15: 49: 53$ & 1,000 \\
\hline Total $^{\circ}$ & $00: 01: 34$ & $30: 57: 11$ & 1,185 \\
\hline
\end{tabular}




\subsection{Figures}

(a)
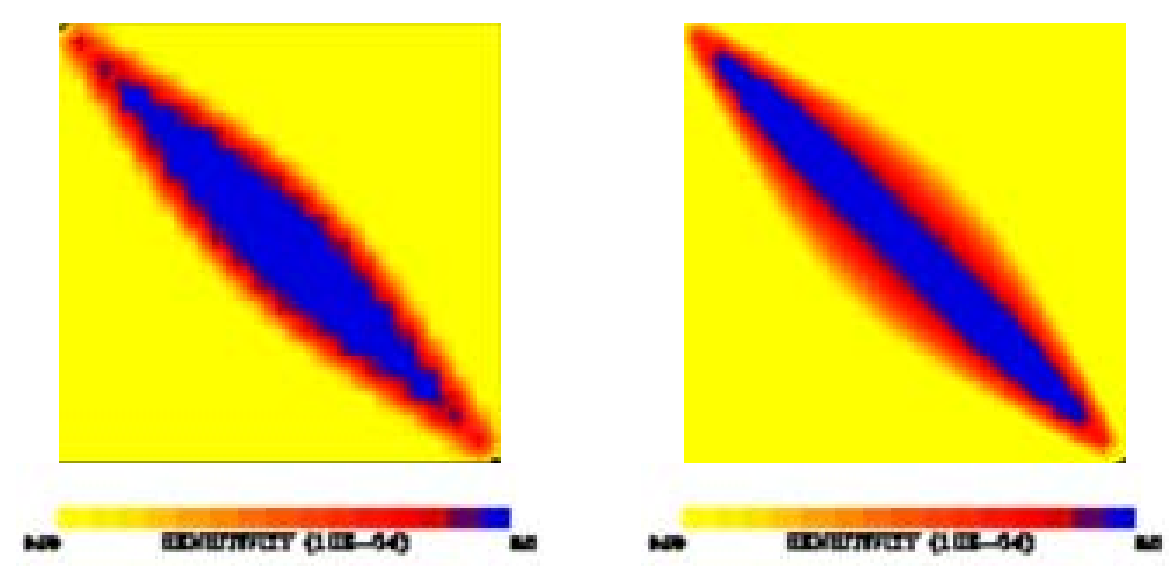

(b)
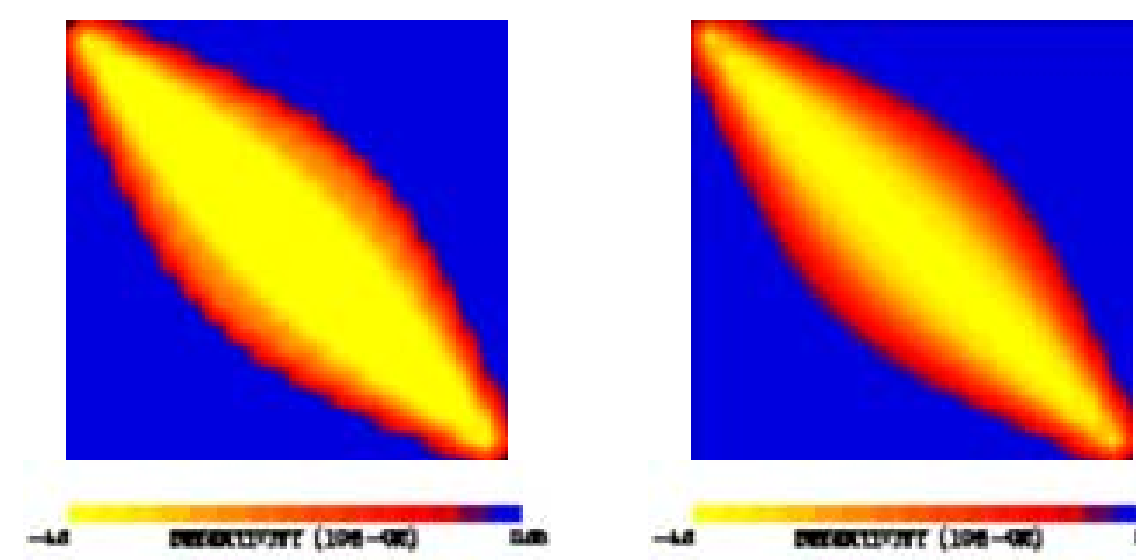

(c)
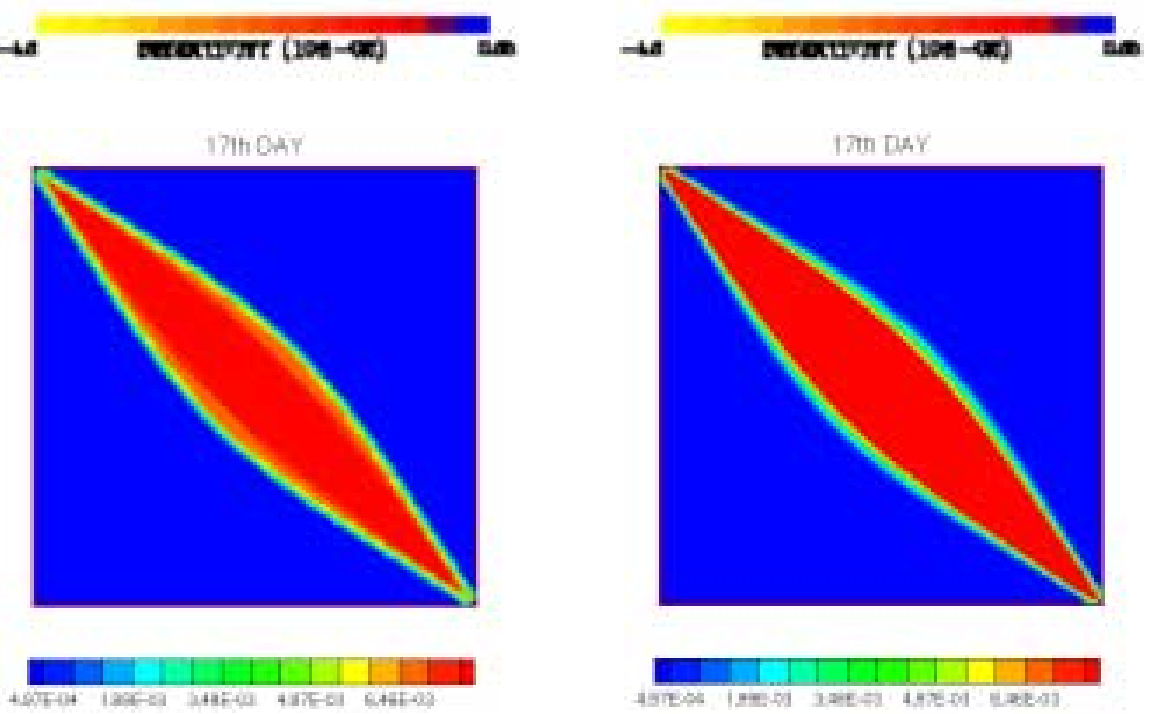

Figure 2.1: Sensitivity comparison between streamline-based analytic approach (Left column) and perturbation based approach (Right column) for quarter five-spot homogeneous example; (a) tracer sensitivity with respect to hydraulic conductivity, (b) tracer sensitivity with respect to porosity, and (c) tracer sensitivity with respect to water saturation. 


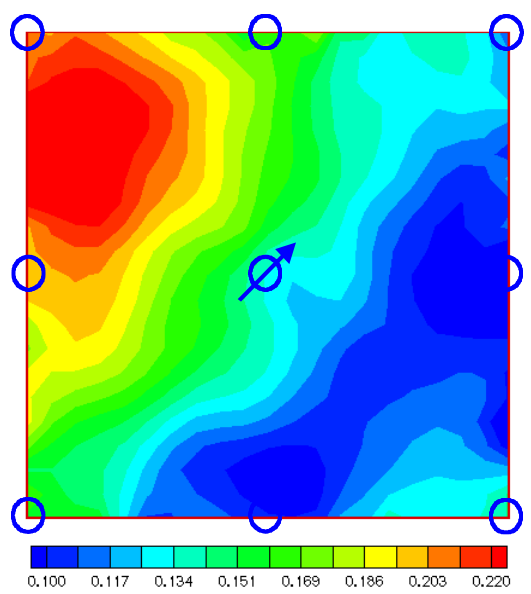

Figure 2.2: Synthetic NAPL saturation distribution generated using a stochastic movingaverage method.

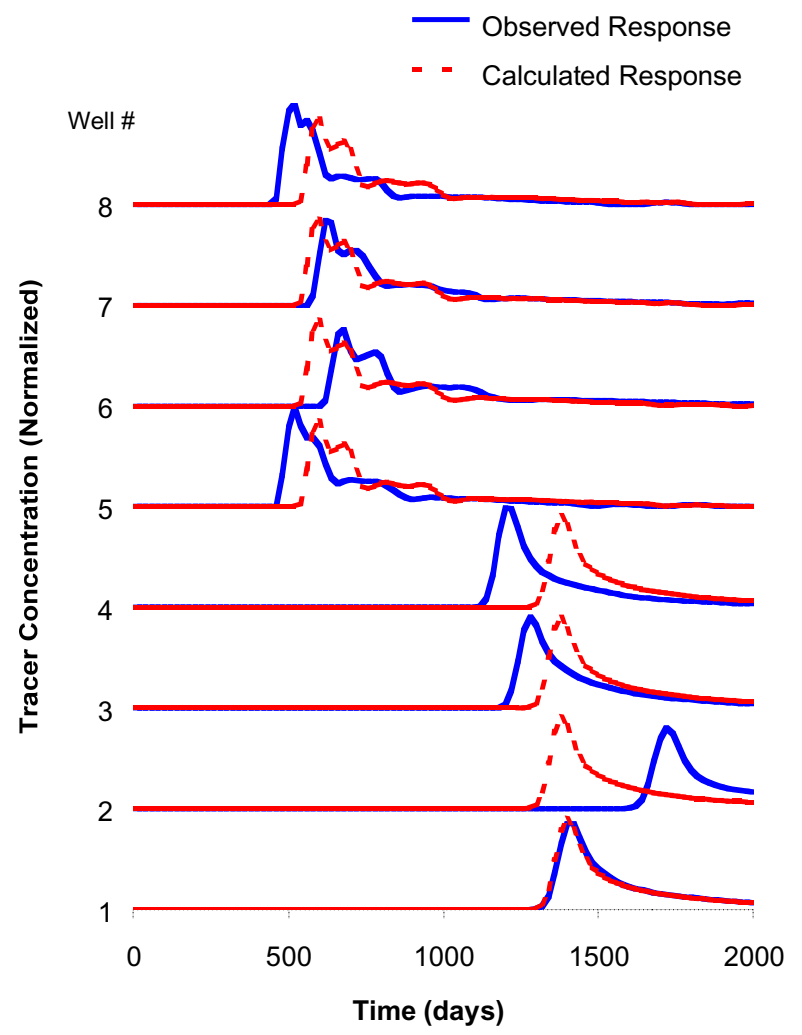

Figure 2.3: Partitioning tracer responses for uniform initial NAPL saturation. (Solid lines represent the tracer responses for the true saturation distribution). 


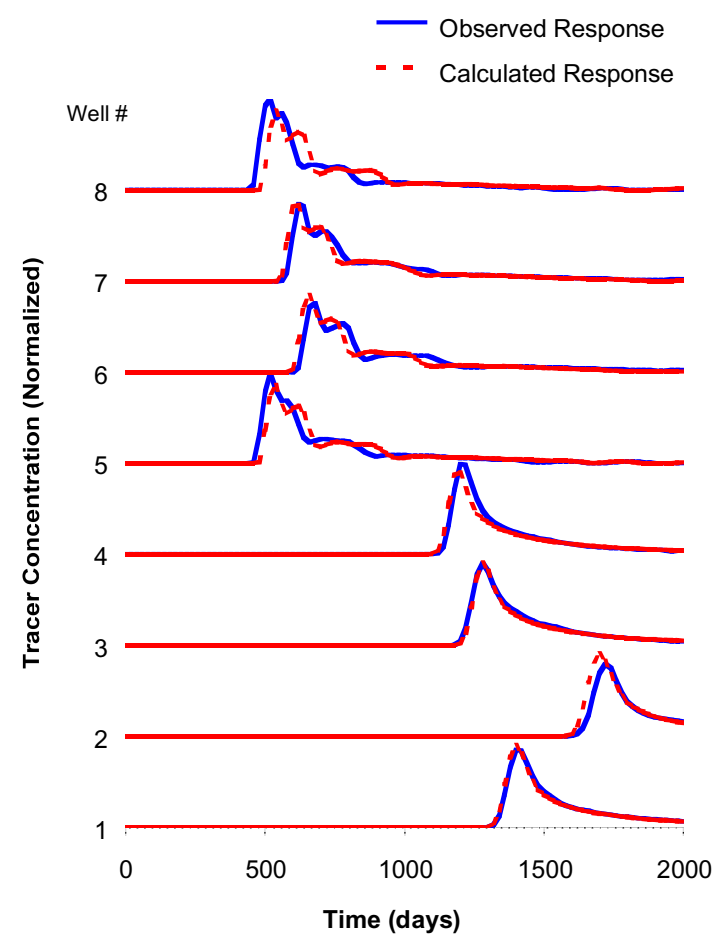

Figure 2.4: Partitioning tracer responses after peak arrival time inversion.
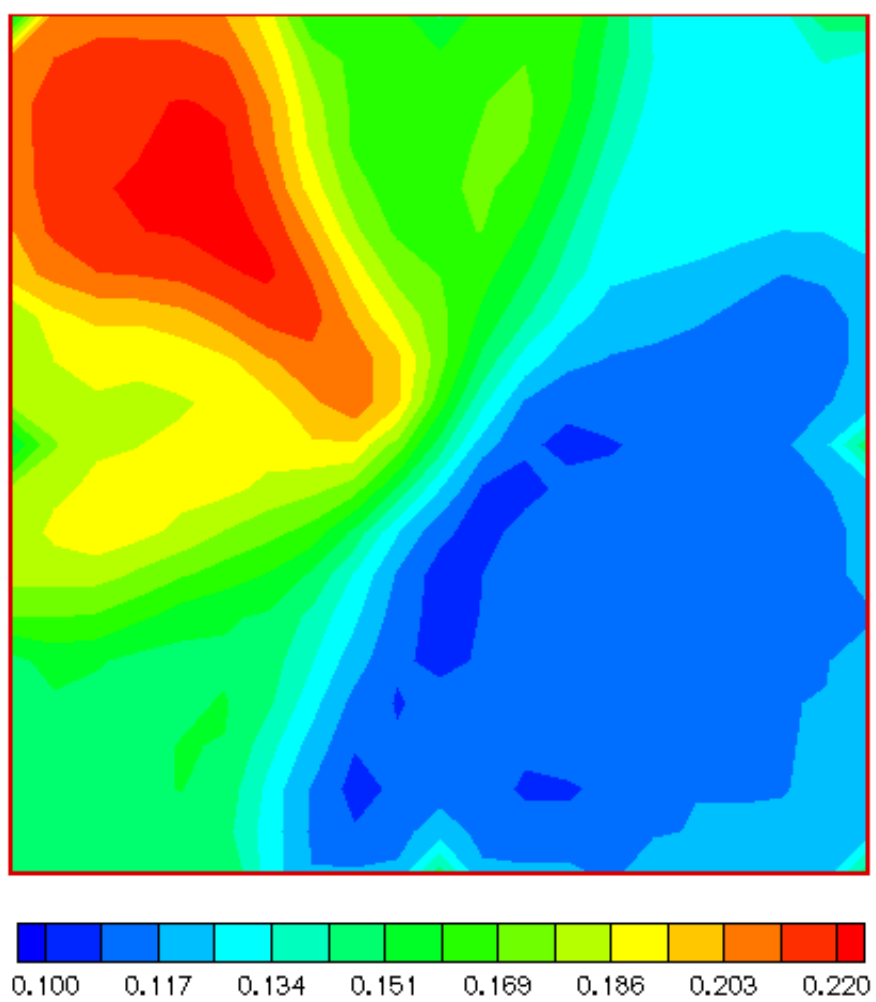

Figure 2.5: Estimated NAPL saturation distribution after peak arrival time inversion. 


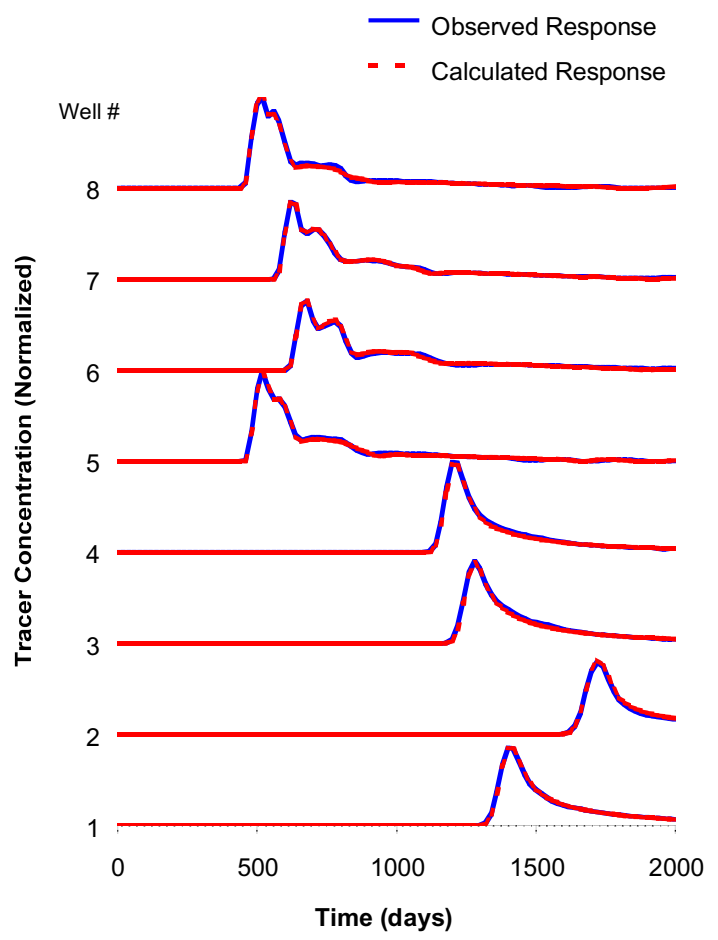

Figure 2.6: Partitioning tracer responses after amplitude inversion.

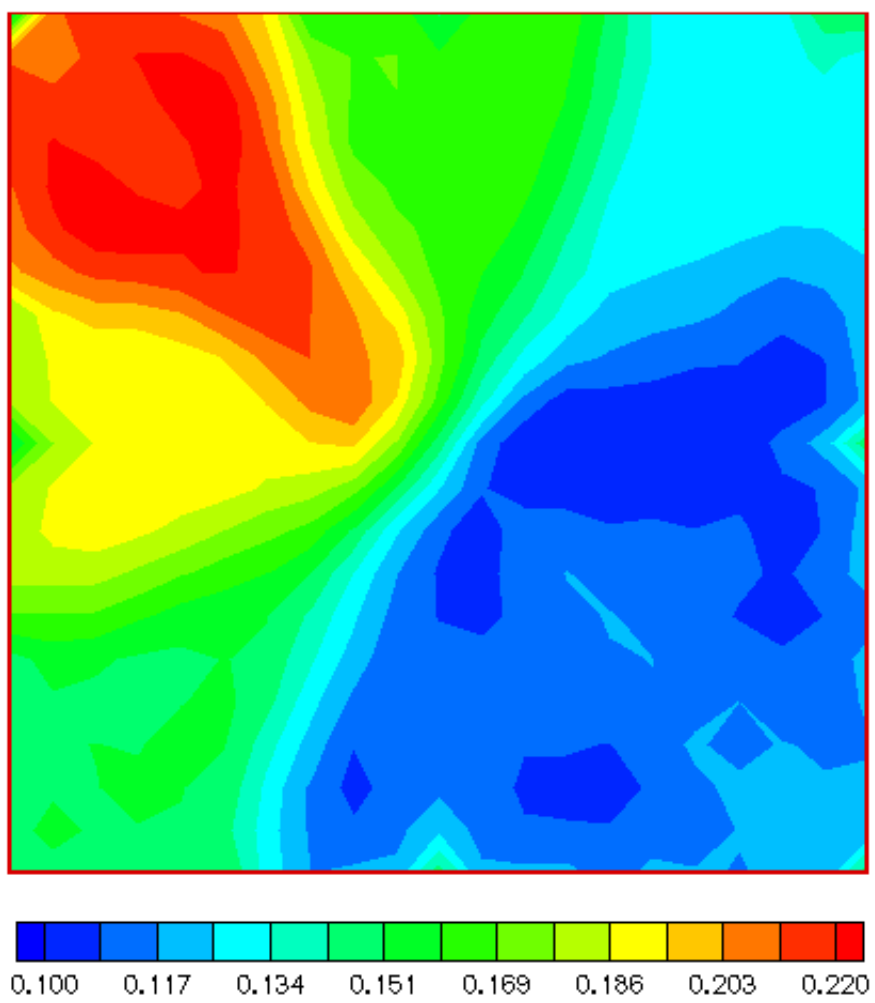

Figure 2.7: Estimated NAPL saturation distribution after amplitude inversion. 


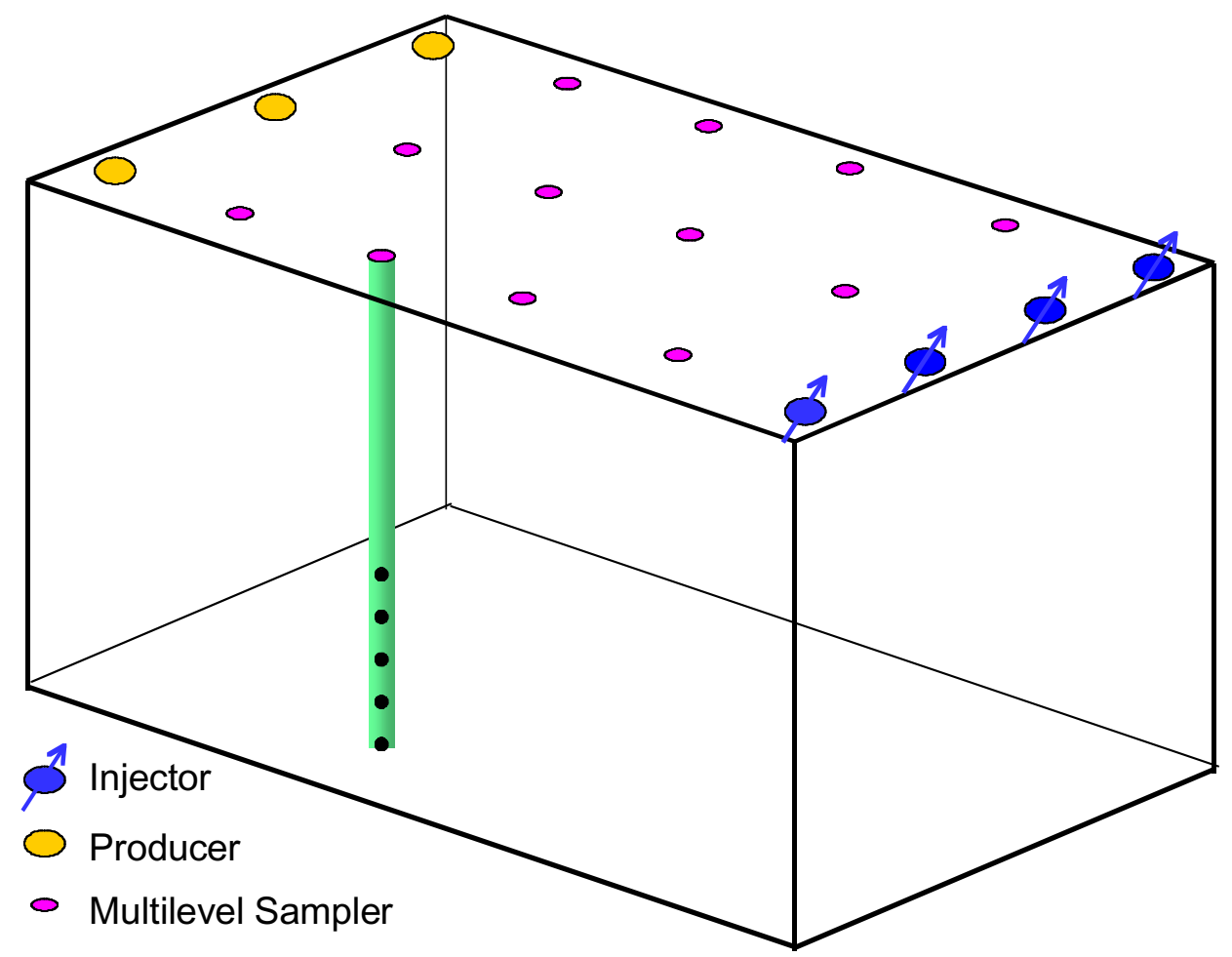

Figure 2.8: Hill Airforce Base test cell diagram.

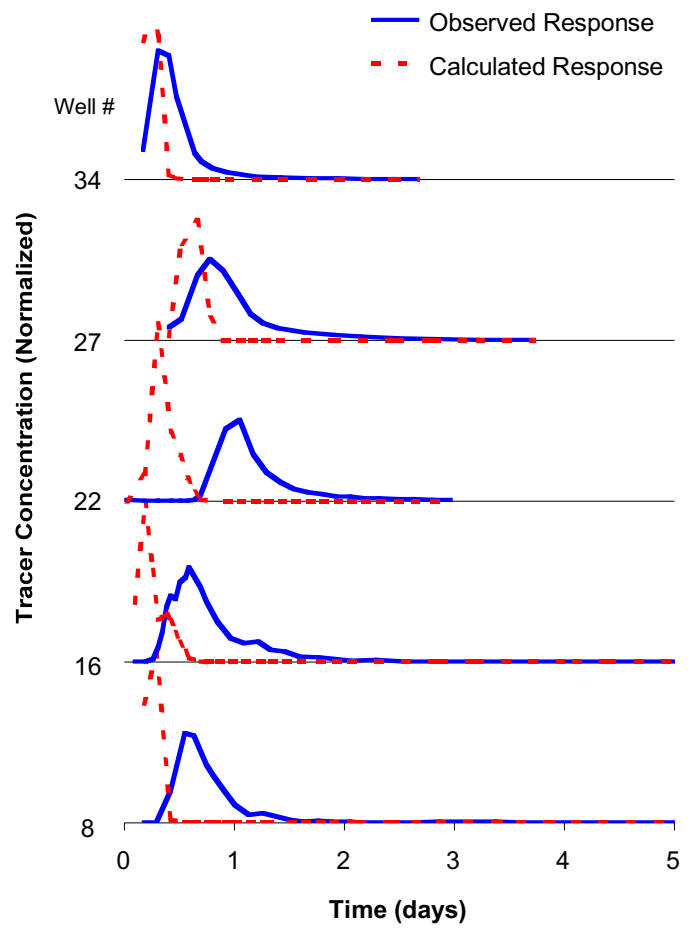

Figure 2.9: Conservative tracer responses at five selected sampling locations for a uniform initial hydraulic conductivity model. 


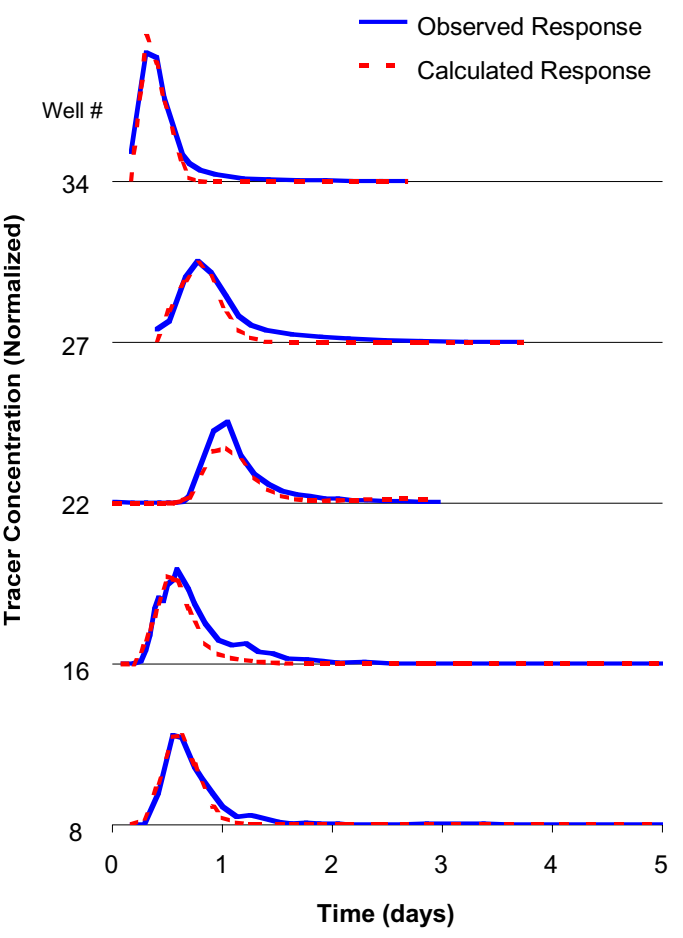

Figure 2.10: Conservative tracer responses at five selected sampling locations for the final hydraulic conductivity model.

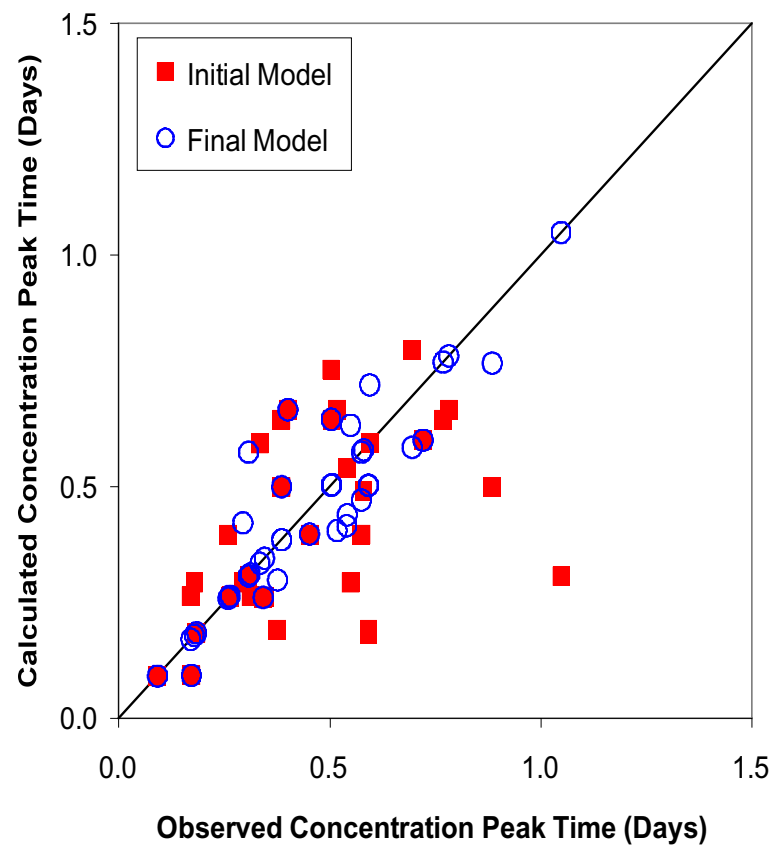

Figure 2.11: Observed and calculated concentration peak arrival times at all sampling locations before and after inversion for hydraulic conductivity. 

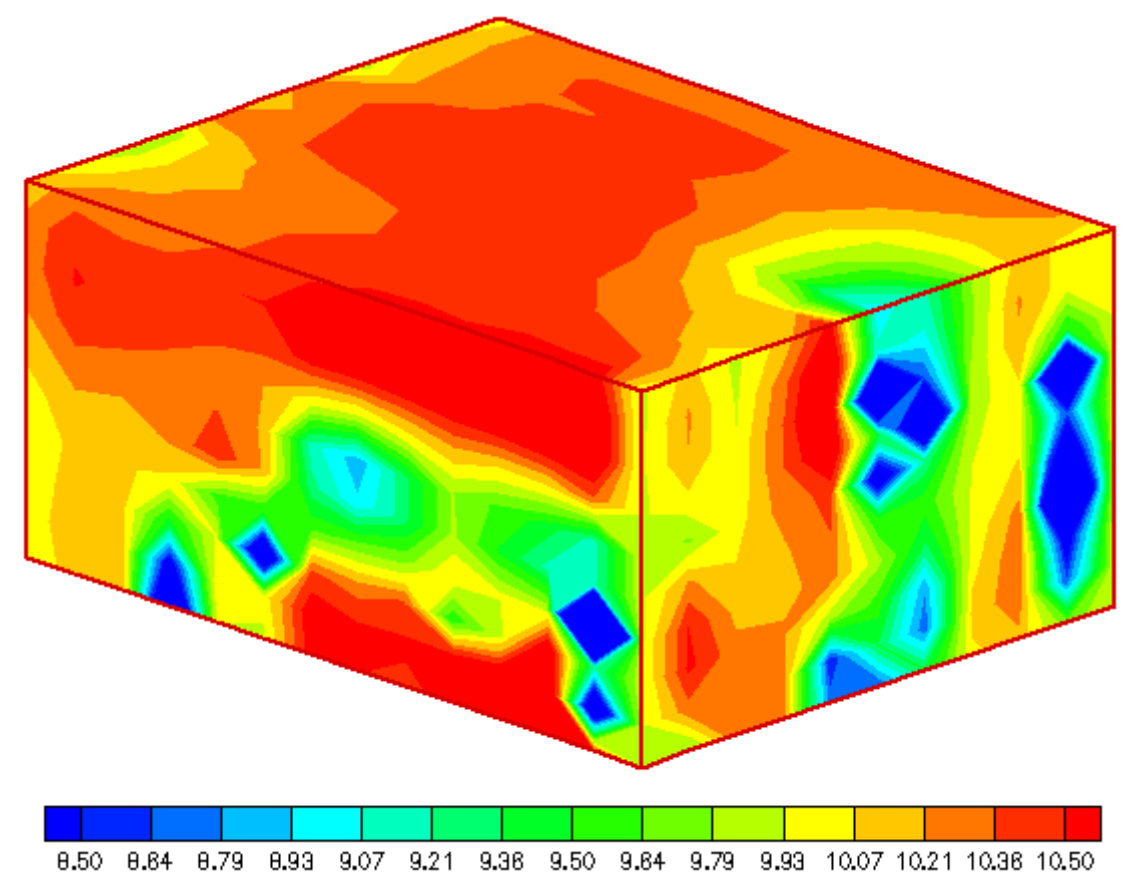

Figure 2.12: Hydraulic conductivity field estimated from the conservative tracer data for the Hill case. (log permeability scale)

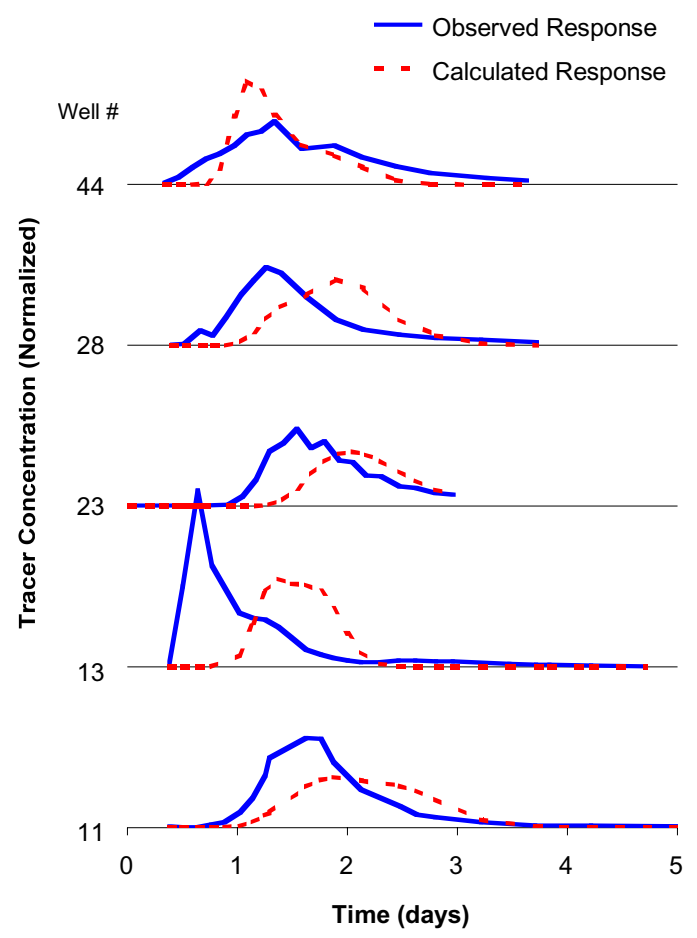

Figure 2.13: Partitioning tracer responses at five selected sampling locations for a uniform initial NAPL saturation model. 


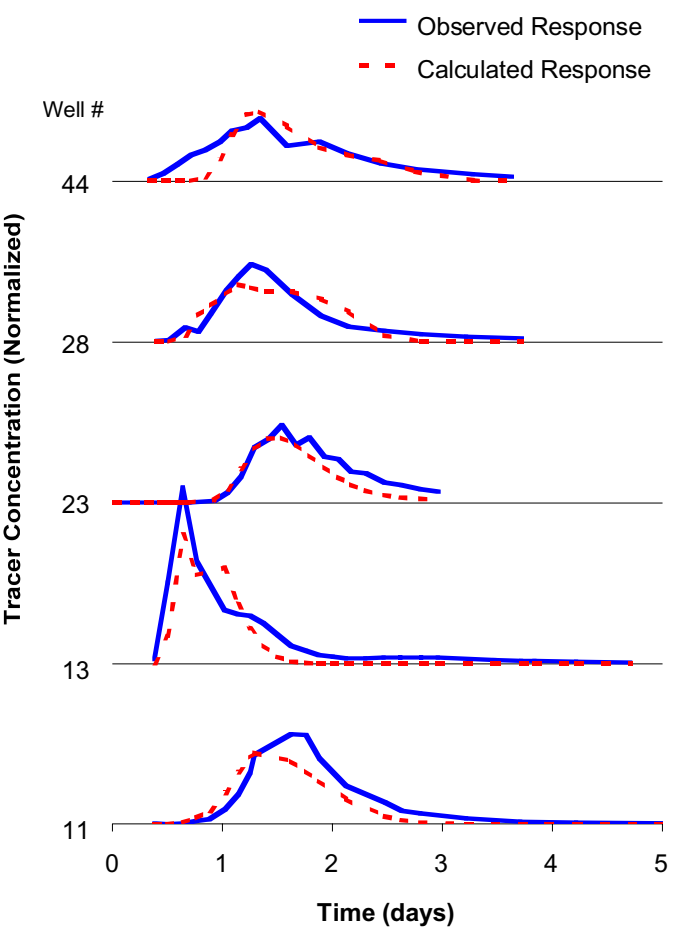

Figure 2.14: Partitioning tracer responses at five selected sampling locations for the final NAPL saturation model.

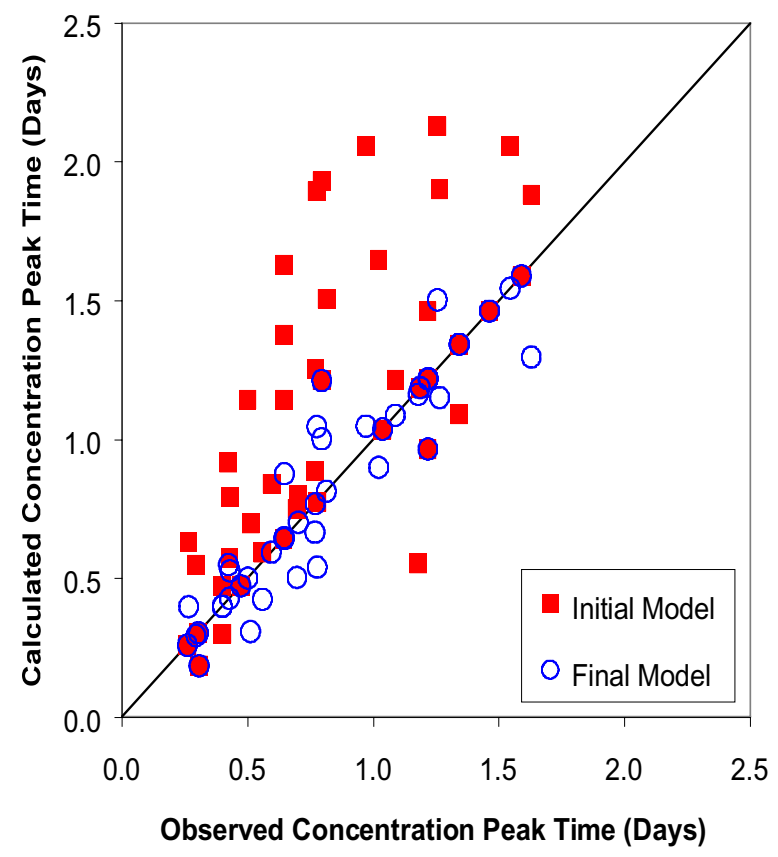

Figure 2.15: Observed and calculated concentration peak arrival times at all sampling locations before and after inversion for NAPL saturation. 


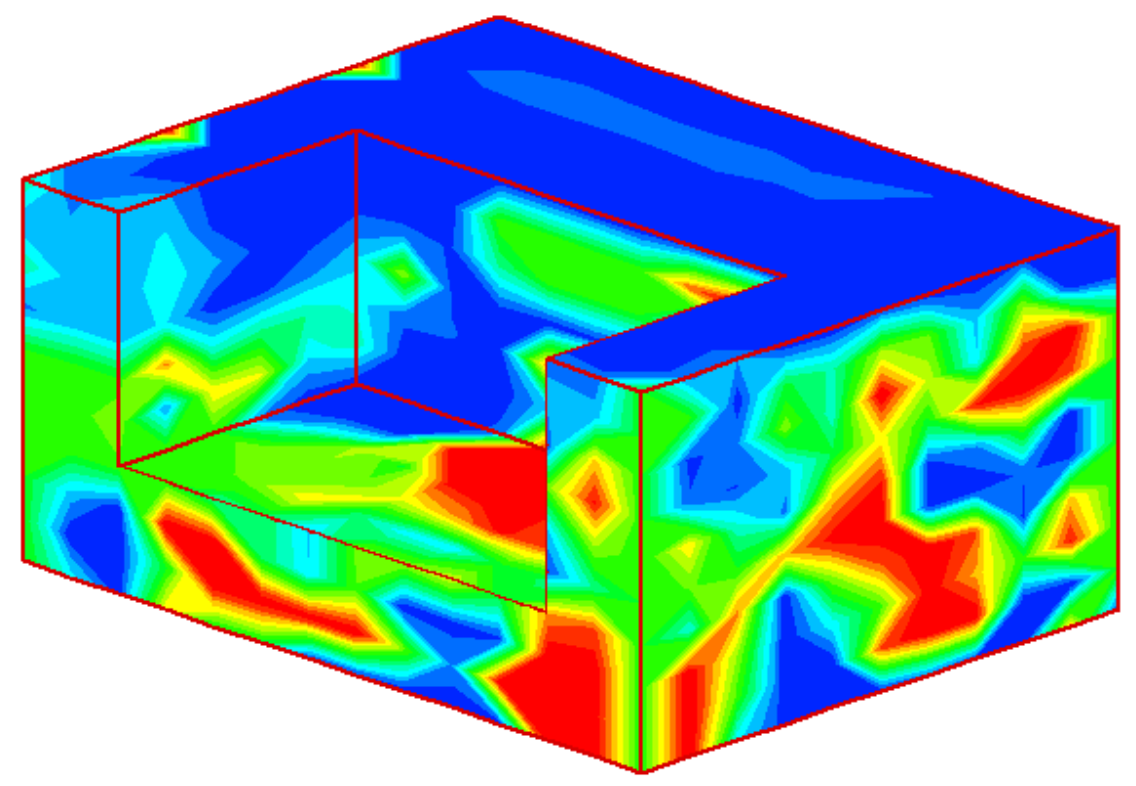

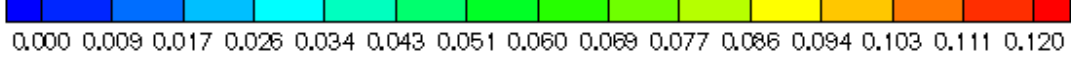

Figure 2.16: NAPL saturation distribution estimated from partitioning tracer data for the Hill case (cut-away view).

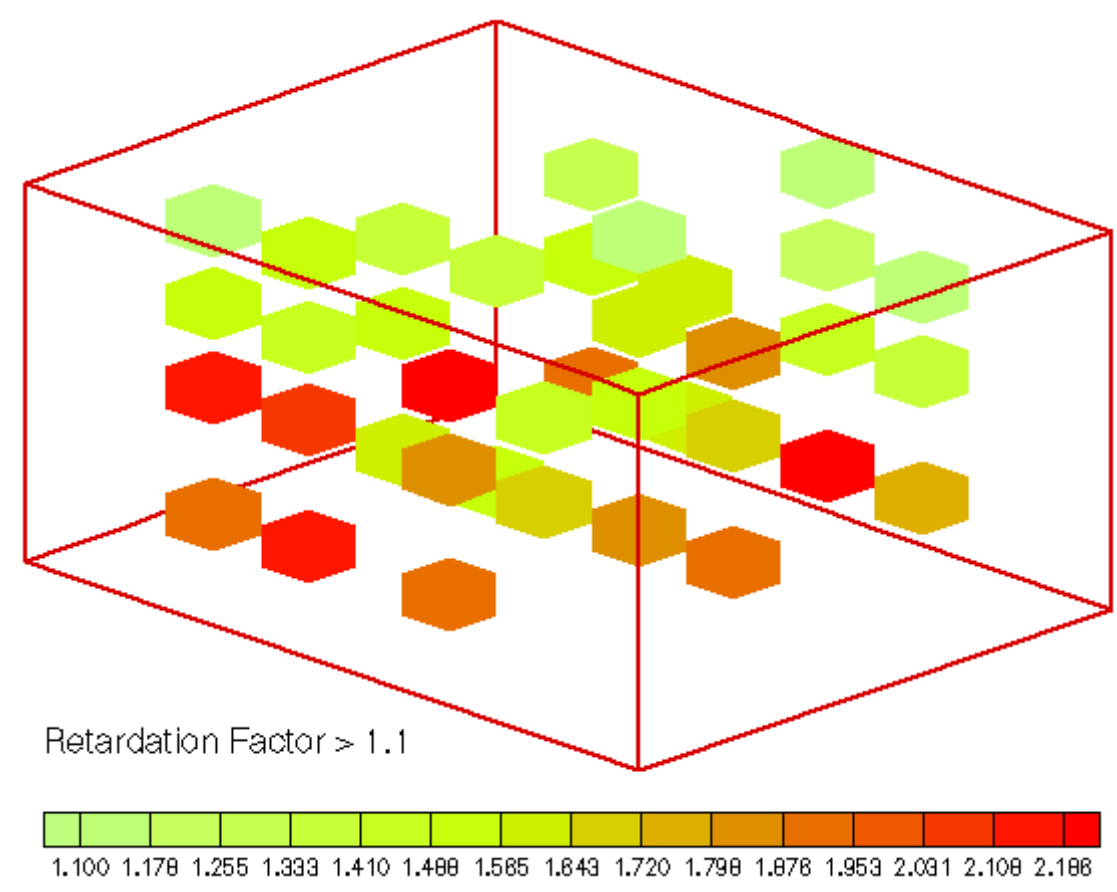

Figure 2.17: Spatial distribution of retardation factors computed at sampling locations. 


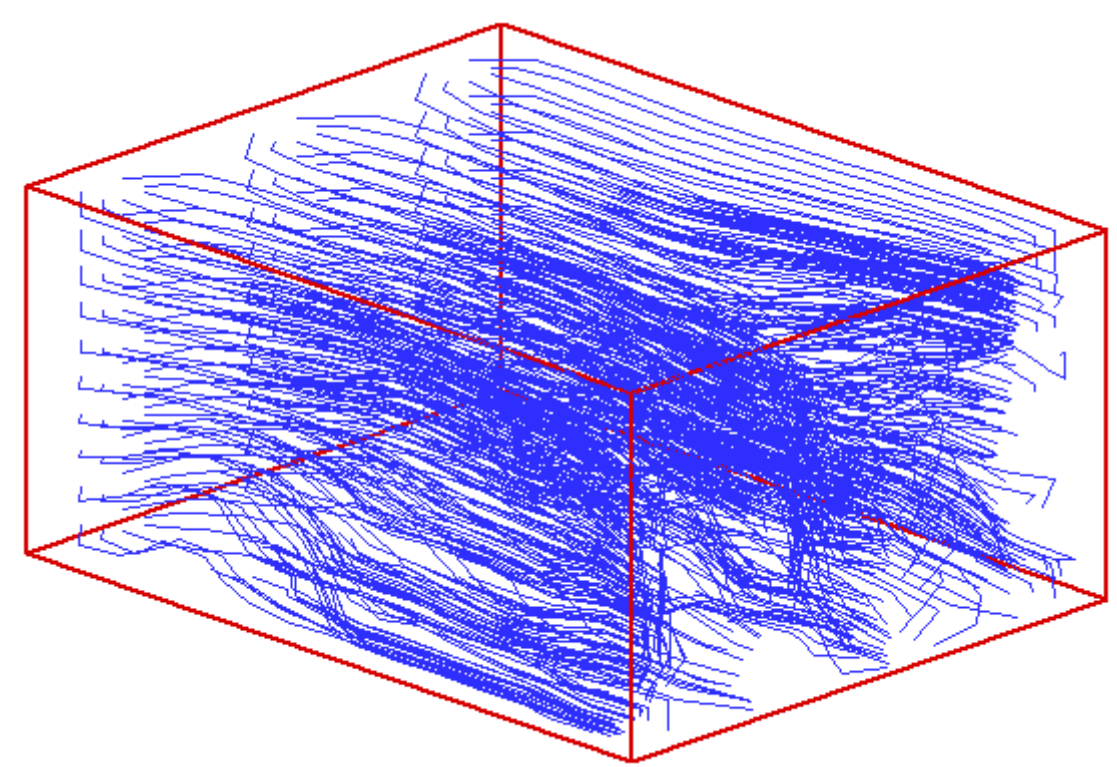

Figure 2.18: 3-D streamline pattern of the final model.
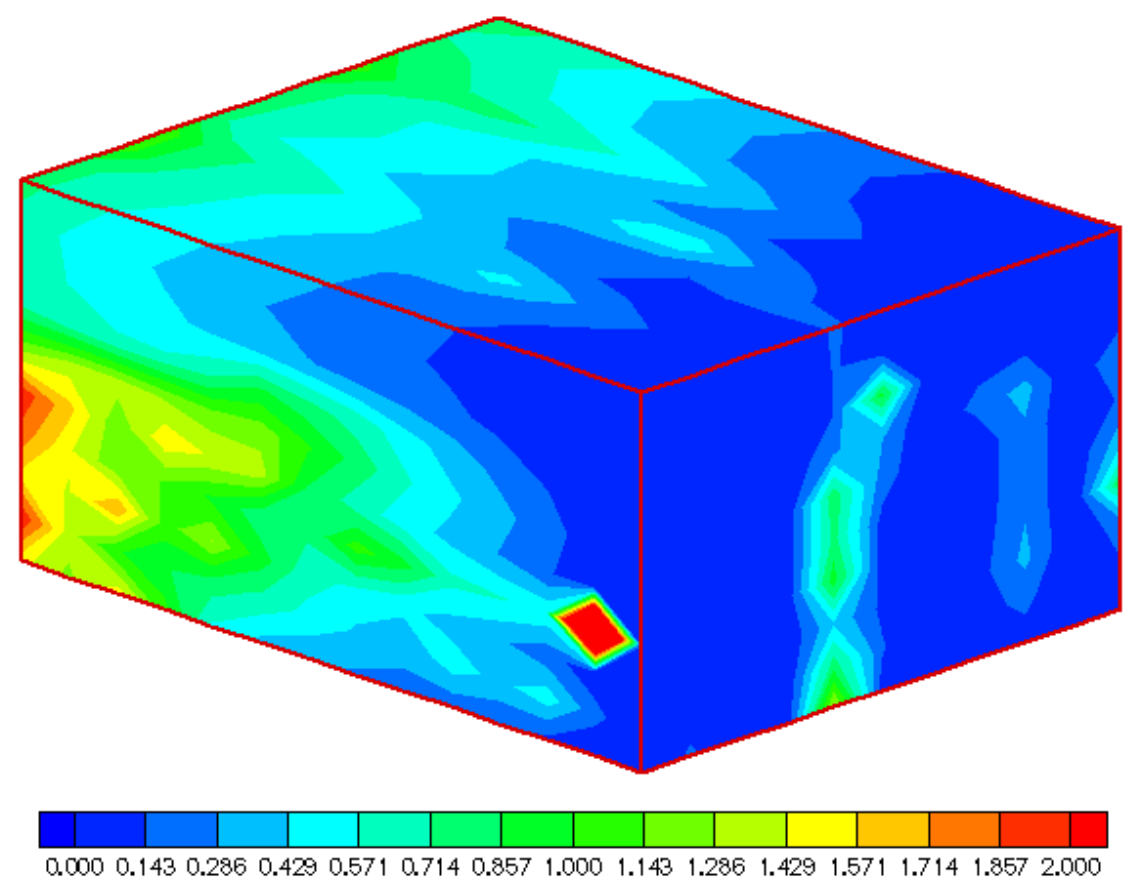

Figure 2.19: Tracer swept volume at various times (days). 


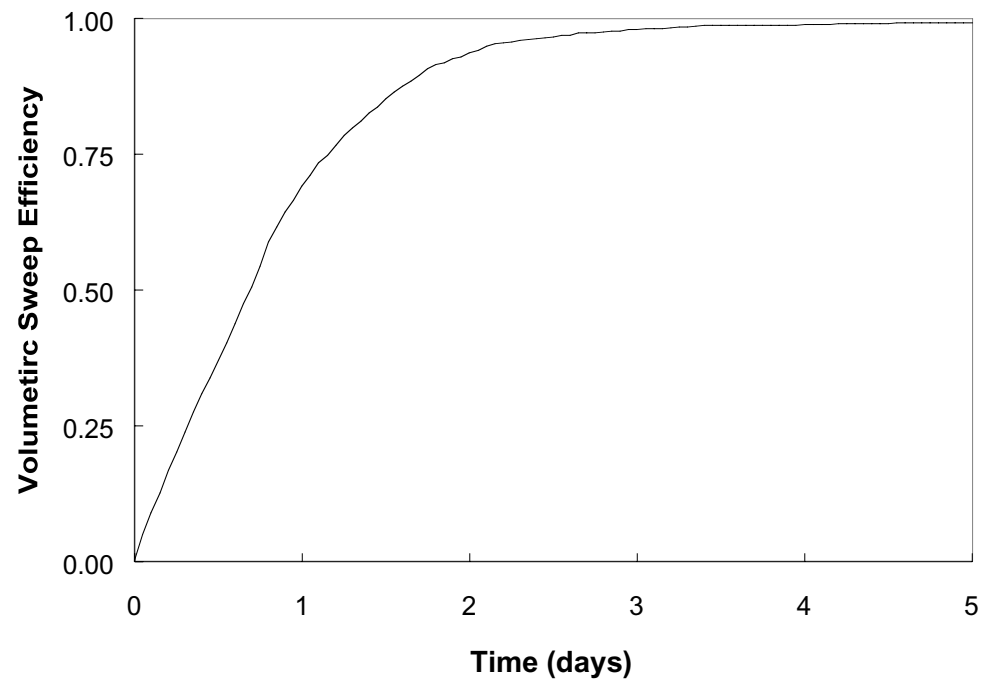

Figure 2.20: Volumetric Sweep Efficiency with function of times (days).

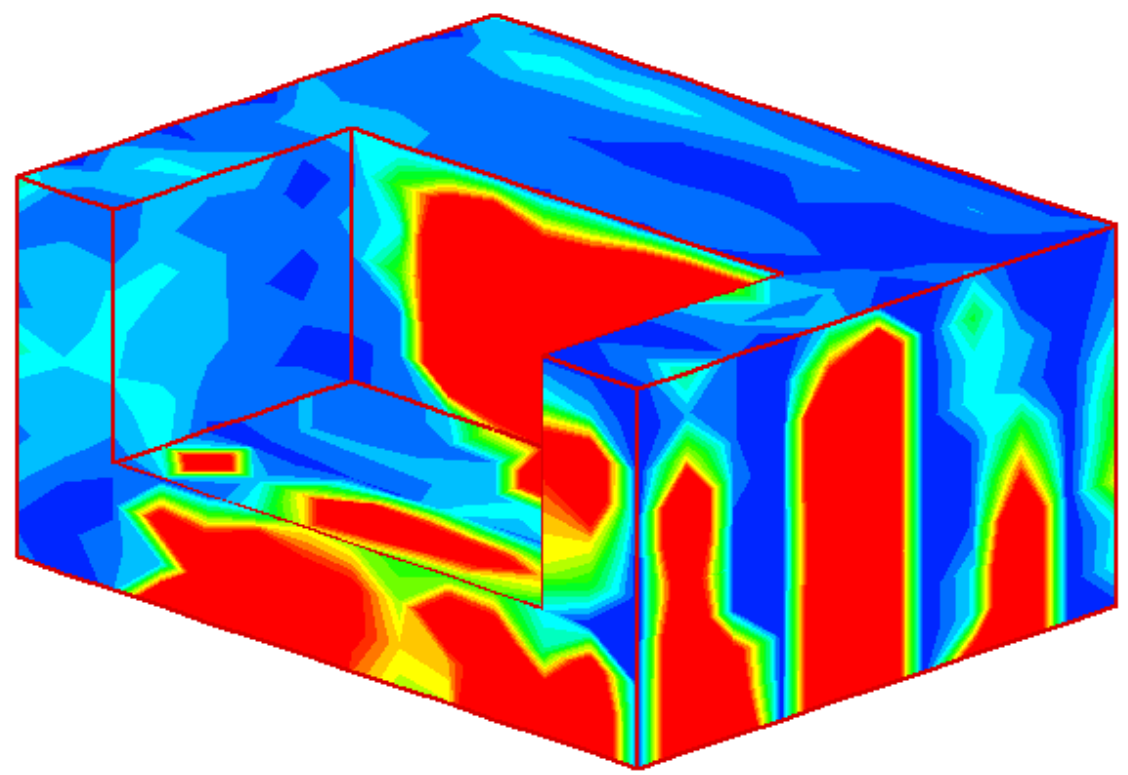

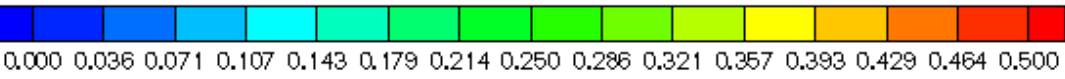

Figure 2.21: Resolution of estimated water saturation. 


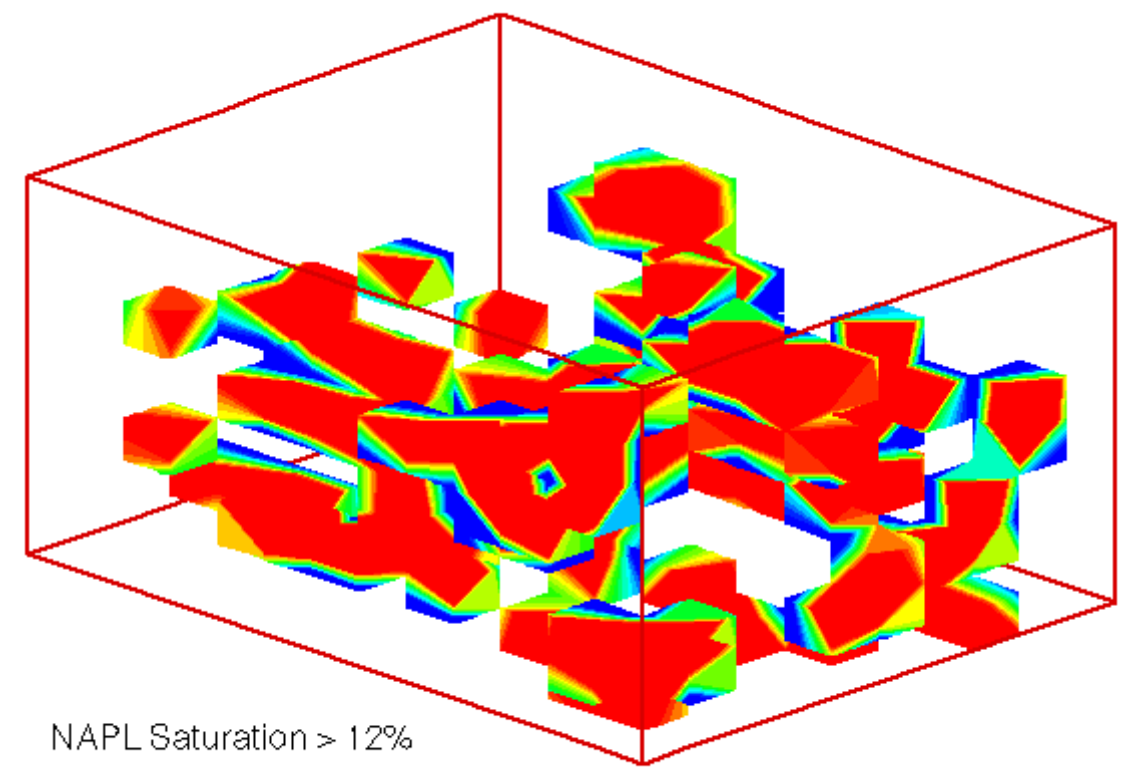

Figure 2.22: NAPL saturation distribution estimated using simulated annealing technique for the Hill case. 


\section{Section 3: Tracer Mass Transfer in Porous Media}

The role of mass transfer limitations has been frequently debated with respect to partitioning tracer tests. Evidence exists (i.e., Whitley, 1997) that partitioning tracer tests can be (and by the informed engineer, are) designed so that local equilibrium occurs for the tracer between the flowing and immobile phases. However, in published literature there is a lack of theoretical and experimental research specifically addressing this topic as it applies to partitioning tracers. This section is intended to be a combination of

1. established mass transfer theory written specifically in terms of partitioning tracers, water, and NAPL,

2. new analysis of partitioning tracer mass transfer using correlations that have been derived for other types of studies, i.e., NAPL dissolution,

3. and a description of the implementation and simulation of tracer mass transfer in UTCHEM, a 3-D transport simulator that is useful for modeling PITTs.

\subsection{Two-resistance Theory}

In this study, mass transfer describes the process by which the partitioning tracer moves from the flowing phase to the immobile phase, and subsequently back to the flowing phase. This transfer of a partitioning tracer between the water and NAPL phases can be described in terms of two-resistance theory. Figure 3.1 illustrates the concept, with some solute concentration distribution between the bulk liquids and their corresponding interfacial concentrations. A rate expression can be written for each phase, where at steady state the rate that the solute reaches the interface from one bulk liquid equals the rate at which the solute enters the second bulk liquid from the interface. 


$$
J=k_{m w}\left(C_{w}-C_{w i}\right)=k_{m N}\left(C_{N i}-C_{N}\right)
$$

where

$$
\begin{aligned}
& k_{m w}=\text { water phase mass transfer coefficient } \\
& k_{m N}=\text { NAPL phase mass transfer coefficient } \\
& C_{w i}=\text { tracer concentration in the water at the interface } \\
& C_{N i}=\text { tracer concentration in the NAPL at the interface }
\end{aligned}
$$

Because the interfacial concentrations are impractical to measure, Whitman (1923) made the suggestion that $C_{N i}$ and $C_{w i}$ could be assumed to be equilibrium concentrations. Whitman (1923) also suggested that the phase resistances are additive. Equilibrium at the interface means equal values of chemical potential in the liquids at the interface and consequently no resistance to transfer across the interface. Treybal (1963) recommends the phrase two-resistance theory to describe these concepts instead of the more popular phrase two-film theory since the theory is equally applicable whether film or penetration theory is used to interpret the $k_{m} \mathrm{~s}$.

Equation (3.1) and the interfacial concentration equilibrium assumption allow an overall mass transfer coefficient to be derived from each of the phase mass transfer coefficients. Figure 3.2 shows a distribution curve for the solute between the water and NAPL phases. Here, $C_{N}{ }^{*}$ is the concentration in the NAPL that would be in equilibrium with $C_{w}$, and $C_{w}{ }^{*}$ is the concentration in the water that would be in equilibrium with $C_{N}$. The equilibrium distribution of the partitioning tracer is linear at dilute concentrations (Dwarakanath, 1997), and the slope of the line is the partition coefficient for the solute between the water and NAPL phases.

Overall two-phase mass transfer coefficients can be defined such that the rate of mass flux from the bulk water to the bulk NAPL phase is 


$$
J=K_{M w}\left(C_{w}-C_{w}^{*}\right)
$$

or alternatively

$$
J=K_{M N}\left(C_{N}^{*}-C_{N}\right)
$$

Note that $K_{M w}$ and $K_{M N}$ are the overall mass transfer coefficients for the two phase system. From the geometry in Fig. 3.2 we can write

$$
\left(C_{N}^{*}-C_{N}\right)=\left(C_{N i}-C_{N}\right)+K_{N}\left(C_{w}-C_{w i}\right)
$$

Substituting equations (3.1) and (3.3) into (3.4) gives

$$
\frac{J}{K_{M N}}=\frac{J}{k_{m N}}+\frac{J K_{N}}{k_{m w}}
$$

and since the fluxes are assumed to be equal then

$$
\frac{1}{K_{M N}}=\frac{1}{k_{m N}}+\frac{K_{N}}{k_{m w}}
$$

A similar derivation using equation (3.2) yields the analogous relationship

$$
\frac{1}{K_{M w}}=\frac{1}{k_{m N} K_{N}}+\frac{1}{k_{m w}}
$$

Equations (3.6) and (3.7) show that the overall coefficients are simply related:

$$
K_{M N}=\frac{K_{M w}}{K_{N}}
$$

This relationship is true only if the equilibrium distribution is linear, as is the case with a partitioning tracer distributed between water and NAPL at dilute concentrations.

\subsection{Estimation of Phase Mass Transfer Resistances}

No known experiments have been completed to measure overall two-phase mass transfer coefficients, (i.e., $K_{M N}$ or $K_{M w}$ ) in saturated porous media. However, estimates can be made for 
the water and NAPL phase resistances and equations (3.6) or (3.7) can be used to estimate the overall two-phase mass transfer resistance.

\subsubsection{Water Phase Resistance}

The water phase resistance to mass transfer has been studied extensively in porous media due to the importance of NAPL dissolution in aquifers. A number of empirical models have recently been formulated to describe mass transfer in saturated media. Imhoff et al. (1997) contains a summary of recently developed models of this type. In these dissolution studies, mass transfer of solute from the NAPL phase is modeled using an equation analogous to equation (3.1)

$$
J=\frac{1}{A_{w N}} \frac{f m}{f t}=-k_{m w}\left(C_{w}-C_{S}\right)
$$

where $A_{w N}$ is the water/NAPL interfacial area, and the interfacial concentration $C_{w i}$ from equation (3.1) is assumed to be equal to $C_{S}$, the equilibrium solubility limit in water. The specific interfacial area $a_{w N}$ is not measured in these studies, so a lumped mass transfer coefficient is calculated

$$
k_{a w}=k_{m w} a_{w N}
$$

Three main empirical models exist in current literature for estimating $k_{a w}$, as established in Miller et al. (1990), Powers et al. (1994), and Imhoff et al. (1994). All three models have been shown to fit laboratory experimental data equally well. For this research, it was important that the chosen model not require inputs that were not typically available for field scale subsurface transport simulations. This characteristic of the model was important for implementation of mass transfer in UTCHEM as described in Section 3.5.

The Imhoff et al. (1994) model was rejected because it includes a parameter which describes a phenomenon the authors call dissolution fingering. Dissolution fingering is to this 
point a phenomenon that is described only at the laboratory scale. The other two models are very similar, with the main difference being that the Powers et al. (1994) model requires the uniformity coefficient of the porous media as input. The Miller et al. (1990) model was more appropriate for this research because it requires only the median grain size, which can be estimated from permeability. The chosen model takes the following form:

$$
S h=\beta_{0} \theta_{w}\left(N_{R e}\right)^{\beta_{1}}(S c)^{\beta_{2}}\left(\theta_{n}\right)^{\beta_{3}}
$$

where

$$
\begin{array}{ll}
S h=\frac{k_{a w} d_{50}^{2}}{D_{w}} & \text { modified Sherwood number } \\
\theta_{w}=\phi S_{w}=\phi\left(1-S_{N}\right) & \text { bulk water content } \\
\theta_{n}=\phi S_{N} & \text { bulk NAPL content } \\
N_{\mathrm{Re}}=\frac{u \rho_{w} d_{50}}{\mu_{w}} & \text { Reynolds number } \\
S_{c}=\frac{\mu_{w}}{\rho_{w} D_{w}} & \text { Schmidt number } \\
\beta_{i} & \text { empirical fitting constant i }
\end{array}
$$

The first three beta values were established in the Miller et al. (1990) paper as

$$
\begin{aligned}
& \beta_{0}=12 \\
& \beta_{1}=0.75 \\
& \beta_{2}=0.50
\end{aligned}
$$

Miller et al. (1990) originally established the fourth fitting parameter, $\beta_{3}$ as 0.60 . However, this value has been questioned in more recent papers, which put the value closer to 1.0. Powers et al. (1994) suggests that the difference lies in the procedure used to create a residual 
NAPL in the columns. The Miller et al. (1990) entrapment procedure involved stirring NAPL into a water-saturated glass bead pack, resulting in an artificially uniform NAPL blob distribution and shape. The Powers et al. (1994) and more recent Imhoff et al. (1998) studies both used the technique of flooding a packed column with NAPL followed by water flood. Both of these studies showed that a $\beta_{3}$ of 1.0 was appropriate for all three models under the more realistic NAPL distributions.

With $\beta_{3}$ equal to 1.0 , the correlation used for this research is as follows:

$$
S h=12 \theta_{w}\left(N_{R e}\right)^{0.75}(S c)^{0.5}\left(\theta_{n}\right)^{1.0}
$$

under the restrictions

$$
\begin{aligned}
& 0.0015<N_{R e}<0.15 \\
& 0.00<\theta_{n}<0.07
\end{aligned}
$$

Note that the original Miller et al. (1990) results were valid only to a minimum volumetric NAPL content of 0.016, but the Imhoff et al. (1998) results extended the range down to 0.00, where no separate NAPL phase existed.

The relative importance of the variables in (3.12) can be more readily seen if the equation is written in dimensional form as follows:

$$
k_{a w}=12 \theta_{w} \frac{\rho_{w}}{\mu_{w}} y^{0.25} D_{w}^{0.5} u_{w}^{0.75} d_{50}^{-1.25} \theta_{\dot{N}}^{1.0}
$$

The density, viscosity, and diffusion coefficient in water will vary little from case to case, so the average velocity, mean grain diameter, and NAPL content are the variables that will have the largest impact on the lumped water phase mass transfer coefficient. 


\subsubsection{NAPL Phase Resistance}

For the NAPL phase resistance, a simple equation is suggested by Treybal (1963) for mass transfer from a rigid liquid sphere (i.e., no internal fluid circulation) into an infinitely dilute fluid:

$$
k_{m N}=\frac{\pi^{2} D_{N}}{3 r_{N}}
$$

where

$$
\begin{aligned}
& D_{N}=\text { tracer diffusion coefficient in the NAPL } \\
& r_{N}=\text { average radius of NAPL spheres }
\end{aligned}
$$

In reality, the NAPL ganglia that exist in porous media are not totally rigid or spherical. However, equation (3.14) should yield a result that is conservatively small, since it does not account for Marangoni effects (interfacial turbulence) that can significantly decrease dispersed phase resistance (Seader and Henley, 1998).

Equation (3.14) provides an estimate of the NAPL phase mass transfer coefficient, but not the specific interfacial area. An estimate of the bulk specific interfacial area between the water and NAPL phases can be made by assuming spherical NAPL blobs in the porous media.

$$
a_{w N}=\frac{A_{w N}}{(\text { bulk } V)}=\frac{A_{w N}}{V_{N}} \frac{V_{N}}{(\text { bulkV) }}=\frac{4 \pi r_{N}^{2}}{\frac{4}{3} \pi r_{N}^{3}} \theta_{N}=\frac{3 \theta_{N}}{r_{N}}
$$

Combining equations (3.14) and (3.15) gives an expression for the NAPL phase lumped mass transfer coefficient.

$$
k_{a N}=k_{m N} a_{w N}=\frac{\pi^{2} D_{N} \theta_{N}}{r_{N}^{2}}
$$


An estimate of the tracer diffusion coefficient, $D_{N}$, in the NAPL can be made using the Wilke-Chang technique, which is essentially an empirical modification of the Stokes-Einstein relation (Reid et. al, 1987):

$$
D_{a b}=\frac{7.4 \times 10^{-8}\left(M_{B}\right)^{0.5} T}{\eta_{b} V_{a}^{0.6}}
$$

where $D_{a b}=$ mutual diffusion coefficient of solute $\mathrm{A}$ in solvent $\mathrm{B}, \mathrm{cm}^{2} / \mathrm{s}$

$M_{b}=$ molecular weight of solvent

$T=$ temperature, $\mathrm{K}$

$\eta_{b}=$ viscosity of solvent $\mathrm{b}, \mathrm{cP}$

$V_{a}=$ molar volume of solute $\mathrm{A}$ at its normal boiling temperature, $\mathrm{mL} / \mathrm{mol}$

Decane and hexanol will be used as an example NAPL/partitioning tracer pair. For decane, $M_{b}=142$ and $\eta_{b}=0.86 \mathrm{cP}$ (at $25 \mathrm{C}$ ). For hexanol, $V_{a}=144 \mathrm{~mL} / \mathrm{mol}$ by the Tyn and Calus method (Reid et. al, 1987). Using equation (3.17), $D_{a b}=1.5 \times 10^{-5} \mathrm{~cm}^{2} / \mathrm{s}$ for hexanol diffusing in decane.

\subsection{Relative Importance of NAPL and Water Phase Resistances}

Equation (3.6) or equation (3.7) can be used to estimate an overall two-phase mass transfer resistance given estimates of the single phase resistances from equations (3.13) and (3.16). The following section details an analysis of the relative contribution of each phase resistance to the overall two-phase resistance. The objective is to determine whether for typical contaminated aquifer conditions one of the phase resistances can be neglected. This analysis is important for two reasons: it provides insight into the dominant mechanisms that govern tracer mass transfer in 
PITTs and it may allow for simpler implementation of mass transfer into existing porous media transport models.

Equations (3.13) and (3.16) contain 8 variables: $\rho_{w}, \mu_{w}, D_{w}, u_{w}, d_{50}, \theta_{N}, D_{N}$, and $r_{N}$. In addition, equation (3.6) contains the partition coefficient, $K_{N}$. A base case was established with typical values for each of the variables. Table 3.1 shows the values for each variable for the base case.

The relative contribution of the NAPL phase resistance to the overall two-phase resistance $K_{M N}$ was calculated using a rearrangement of equation (3.6):

$$
\frac{\frac{1}{k_{m N}}}{\frac{1}{K_{M N}}}=\frac{K_{M N}}{k_{m N}}=\overline{1}+\frac{k_{m N} K_{N}}{k_{m w}} \underbrace{-1}
$$

Note that equation (3.18) can also be written in terms of $K_{M w}$, where

$$
\frac{\frac{1}{k_{m N}}}{\frac{1}{K_{M w}}}=\frac{K_{M w}}{k_{m N}}=\frac{-1}{K_{N}}+\frac{k_{m N}}{k_{m w}} y^{-1}
$$

Equation (3.18) was used in the analysis since the Damkohler number is proportional to $K_{M N}$ (or to the fraction $\left.K_{M w} / K_{N}\right)$. The derivation and importance of the Damkohler number is explained in detail in section 3.4. The small variations in $\rho_{w}, \mu_{w}, D_{w}$, and $D_{N}$ for typical scenarios will not have a significant impact on the overall two-phase mass transfer coefficient and were held constant in this analysis. The parameters $d_{50}, \theta_{N}, D_{N}$, and $r_{N}$ were varied one at a time over the ranges specified in Table 3.1 with the remaining variables set at base case values. Figures 3.33.6 show the percent relative contribution of the NAPL phase resistance to the overall two-phase resistance for each case. 
The maximum contribution from the NAPL phase resistance was approximately $6 \%$, for the minimum grain diameter sand. The NAPL phase resistance increases as the square of the NAPL sphere diameter (equation (3.16)). However, for the typical range of sphere diameters shown in Fig. 3.5, the NAPL phase resistance was minimal (2\%). In general, the NAPL phase resistance showed a minimal contribution to the overall two-phase resistance over the given variable ranges.

The partition coefficient, $K_{N}$, was 30 for the base case. In the field, with typical average NAPL saturations less than $3 \%$, a $K_{N}$ of 30 is near the minimum acceptable value for $K_{N}$. Equation (3.18) shows that the relative contribution of the NAPL phase resistance to the overall two-phase resistance would decrease nearly proportionately with increasing $K_{N}$, so the NAPL phase resistance would have even less impact on the overall resistance for partitioning tracers with higher values of $K_{N}$.

\subsection{One Dimensional Transport Model with Mass Transfer}

\subsubsection{Determination of Relevant Parameters}

The following equation describes 1-D partitioning tracer transport in a porous medium that contains an immobile NAPL:

$$
\frac{f\left(\phi S_{w} C_{w}+\phi S_{N} C_{N}\right)}{f t}+\frac{f\left(q_{w} C_{w}\right)}{f x}-\frac{f}{f x} \bar{\phi} S_{w} D_{L} \frac{f C_{w}}{f x} \sqrt{ }=0
$$

where

$$
\begin{aligned}
& q_{w}=\text { Darcy flux of water phase } \\
& D_{L}=\text { dispersion coefficient }
\end{aligned}
$$


If the porosity, NAPL saturation, dispersivity coefficient, and Darcy flux are constant, then they can be brought outside the differential. Dividing by the porosity and water saturation yields

$$
\frac{f C_{w}}{f t}+\frac{S_{N}}{S_{w}} \frac{f C_{N}}{f t}+u \frac{f C_{w}}{f x}-D_{L} \frac{f^{2} C_{w}}{f x^{2}}=0
$$

where $u$ is now the average interstitial velocity of the aqueous phase.

From equation (3.2), first order, linear, reversible solute mass transfer between the water and NAPL phases can be written in terms of the overall two-phase mass transfer coefficient:

$$
J=\frac{1}{A_{w N}} \frac{d m}{d t}=K_{M w}\left(C_{w}-C_{w}^{*}\right)
$$

where $C_{w}^{*}$ is the hypothetical concentration of tracer in the water phase that would be in equilibrium with the bulk NAPL phase. Note that solute flux out of the aqueous phase into the NAPL is positive. From the definition of partition coefficient, $K_{N} C_{w}^{*}=C_{N}$ and equation (3.22) becomes

$$
\frac{d m}{d t}=K_{M w} A_{w N}\left(C_{w}-\frac{C_{N}}{K_{N}}\right)
$$

Equation (3.23) can be rewritten in terms of the change in tracer concentration in the NAPL phase:

$$
\frac{d m}{d t}=V \phi S_{N} \frac{f C_{N}}{f t}=K_{M w} A_{w N}\left(C_{w}-\frac{C_{N}}{K_{N}}\right)
$$

where $V$ is a unit bulk volume of porous medium.

Rearranging,

$$
\frac{f C_{N}}{f t}=\frac{K_{M w} A_{w N}}{V \phi S_{N}}\left(C_{w}-\frac{C_{N}}{K_{N}}\right)
$$


The contact surface area divided by the unit bulk volume is the bulk, water-NAPL specific interfacial area, $a_{w N}$ :

$$
\frac{f C_{N}}{f t}=\frac{K_{M w} a_{w N}}{\phi S_{N}}\left(C_{w}-\frac{C_{N}}{K_{N}}\right)
$$

A lumped overall two-phase mass transfer coefficient is given by

$$
K_{a w}=K_{M w} a_{w N}
$$

so

$$
\frac{f C_{N}}{f t}=\frac{K_{a w}}{\phi S_{N}}\left(C_{w}-\frac{C_{N}}{K_{N}}\right)
$$

Equations (3.21) and (3.28) can then be analyzed to determine relevant parameters for the 1-D partitioning tracer transport in porous media containing NAPL, with linear, reversible tracer mass transfer between the water and NAPL.

Linear scale factors are used such that for two variables $A$ and $B$,

$$
\begin{aligned}
& A=A_{1} A_{D}+A_{2} \\
& B=B_{1} B_{D}+B_{2} \\
& \frac{f A}{f B}=\frac{A_{1} f A_{D}}{B_{1} f B_{D}} \\
& \frac{f^{2} A}{f B^{2}}=\frac{A_{1}}{B_{1}^{2}} \frac{f^{2} A_{D}}{f B_{D}^{2}}
\end{aligned}
$$

where

$A_{1}, A_{2}, B_{1}, B_{2}$ are scale factors

$A_{D}, B_{D}$ are dimensionless variables

Using (3.29) as a guide, equations (3.21) and (3.28) can be rewritten as 


$$
\begin{aligned}
& \frac{C_{1, w}}{t_{1}} \frac{f C_{D, w}}{f t_{D}}+\frac{S_{N}}{S_{w}} \frac{C_{1, N}}{t_{1}} \frac{f C_{D, N}}{f t_{D}}+u \frac{C_{1, w}}{x_{1}} \frac{f C_{D, w}}{f x_{D}} \\
& -D_{L} \frac{C_{1}}{x_{1}^{2}} \frac{f^{2} C_{D, w}}{f x_{D}^{2}}=0
\end{aligned}
$$

and

$$
\frac{C_{1, N}}{t_{1}} \frac{f C_{D, N}}{f t_{D}}=\frac{K_{a w}}{\phi S_{N}}\left(C_{D, w} C_{1, w}-\frac{C_{D, N} C_{1, N}}{K_{N}}\right)
$$

where the $A_{2}$ terms have been set to zero to avoid adding terms to the equation.

Then let

$$
\begin{aligned}
& t_{1}=\frac{x_{L}}{u}, t_{D}=\frac{t u}{x_{L}} \\
& x_{1}=x_{L}, x_{D}=\frac{x}{x_{L}} \\
& C_{1, N}=K_{N} C_{1, w}
\end{aligned}
$$

where $x_{L}$ is location of effluent sampling point.

Substituting the conditions (3.32) - (3.34) into equations (3.30) and (3.31) and rearranging yields

$$
\begin{aligned}
& \frac{f C_{D, w}}{f t_{D}}+K_{N} \frac{S_{N}}{S_{w}} \frac{f C_{D, N}}{f t_{D}}+\frac{f C_{D, w}}{f x_{D}}-\frac{D_{L}}{u x_{L}} \frac{f^{2} C_{D, w}}{f x_{D}^{2}}=0 \\
& \left.\frac{f C_{D, N}}{f t_{D}}=-\frac{K_{a w}}{K_{N} \phi S_{N}} \sqrt{\frac{x_{L}}{u}} \sqrt{ }\right\rfloor\left(C_{D, w}-C_{D, N}\right)
\end{aligned}
$$

Recognizing that the parameter groups are arranged in terms of the retardation factor $\left(R_{f}\right)$, the Peclet number $\left(N_{P e}\right)$, and the Damkohler number $\left(N_{D a}\right)$, equations (3.35) and (3.36) can be finally expressed as 


$$
\begin{aligned}
& \frac{f C_{D, w}}{f t_{D}}+\left(R_{f}-1\right) \frac{f C_{D, N}}{f t_{D}}+\frac{f C_{D, w}}{f x_{D}}-\frac{1}{N_{P e}} \frac{f^{2} C_{D, w}}{f x_{D}^{2}}=0 \\
& \frac{f C_{D, N}}{f t_{D}}=N_{D a}\left(C_{D, w}-C_{D, N}\right)
\end{aligned}
$$

where

$$
\begin{aligned}
& R_{f}=1+\frac{K_{N} S_{N}}{S_{w}} \\
& N_{P e}=\frac{u x_{L}}{D_{L}} \\
& N_{D a}=\frac{-K_{a w}}{K_{N} \phi S_{N}} \sqrt[4]{\frac{x_{L}}{u}} \sqrt{ }
\end{aligned}
$$

Note that the retardation factor is the same as that defined in the method of moment equations of Section 2. The Peclet number quantifies the ratio of advective to dispersive tracer transport. The Damkohler number is defined as the ratio of the characteristic time for advection (mean residence time) to the characteristic time for mass transfer.

Equation (3.13) can be substituted into equation (3.41), assuming negligible contribution of the NAPL phase resistance, to yield the Damkohler number in terms of dimensional variables:

$$
N_{D a}=12 \theta_{w} \frac{\rho_{w}}{\mu_{w}} y^{0.25} \frac{D_{w}^{0.5} x_{L}}{K_{N} u^{0.25} d_{50}^{1.25}}
$$

Equation (3.42) is a very important result, because it indicates that the Damkohler number is independent of NAPL saturation, and is only weakly dependent on velocity. The NAPL saturation divided out of the equation because $\beta_{3}=1.0$ in equation (3.13). However, even if $\beta_{3}$ were not exactly equal to 1.0 , the dependence on saturation would still be weak. This result is 
unique to two-phase partitioning, since the volumetric NAPL content would not appear in the denominator of equation (3.41) for single-phase mass transfer (e.g., NAPL dissolution).

\subsubsection{Determining Zero and First Moments}

Beginning with equations (3.37) and (3.38), the following initial and boundary conditions are applied:

$$
\begin{aligned}
& C_{D, N}\left(x_{D}, 0\right)=0 \\
& C_{D, w}\left(x_{D}, 0\right)=0 \\
& C_{D, w}\left(0, t_{D}\right)=\delta\left(t_{D}\right) \\
& C_{D, w}\left(\times, t_{D}\right)=0
\end{aligned}
$$

Initial condition (3.45) includes the Dirac delta function that provides an initial tracer pulse with unit integral area.

Taking the Laplace transform of equations (3.37) and (3.38) with respect to time yields

$$
\begin{aligned}
& s \bar{C}_{D, w}-C_{0, w}+\left(R_{f}-1\right)\left(s \bar{C}_{D, N}-C_{0, N}\right)+\frac{f \bar{C}_{D, w}}{f x_{D}} \\
& -\frac{1}{N_{P e}} \frac{f^{2} \bar{C}_{D, w}}{f x_{D}^{2}}=0 \\
& s \bar{C}_{D, N}-C_{0, N}=N_{D a}\left(\bar{C}_{D, w}-\bar{C}_{D, N}\right)
\end{aligned}
$$

and conditions (3.43) through (3.46) are transformed to

$$
\begin{aligned}
& \bar{C}_{D, N}\left(x_{D}, 0\right)=0 \\
& \bar{C}_{D, w}\left(x_{D}, 0\right)=0 \\
& \bar{C}_{D, w}(0, s)=1 \\
& \bar{C}_{D, w}(\times, s)=0
\end{aligned}
$$


Note that $\bar{C}$ represents the Laplace transform of concentration with respect to time and $s$ is the Laplace variable. Combining (3.49), (3.50), and (3.48) with (3.47) yields

$$
\beta(s) \bar{C}_{D, w}+\frac{f \bar{C}_{D, w}}{f x_{D}}-\frac{1}{N_{P e}} \frac{f^{2} \bar{C}_{D, w}}{f x_{D}^{2}}=0
$$

where

$$
\beta(s)=s+\left(R_{f}-1\right)\left(\frac{s N_{D a}}{s+N_{D a}}\right)
$$

Equation (3.53) is now a linear partial differential equation, and can be solved using boundary conditions (3.51) and (3.52) to yield:

$$
\bar{C}_{D, w}=\exp \frac{N_{P e}-\sqrt{N_{P e}^{2}+4 N_{P e} \beta(s)}}{2} x_{D} \sqrt{ }
$$

This solution is in Laplace space, and would be difficult if not impossible to invert analytically. Fortunately, analytical inversion is not necessary for determining the first and second moments. Aris (1958) gives an equation for determining temporal moments from a solution that is in Laplace space:

$$
m_{n}=(-1)^{n}\left(\frac{f^{n} \bar{C}}{f s^{n}}\right)_{s} \bullet 0
$$

where $m_{n}$ is the $\mathrm{n}^{\text {th }}$ temporal moment.

Applying (3.56) to equation (3.55) with $x_{D}=1$, for $\mathrm{n}=0$ and $\mathrm{n}=1$ yields:

$$
\begin{aligned}
& m_{0}=1 \\
& m_{1}=R_{f}
\end{aligned}
$$

Equation (3.58) is very important because it shows that the mean residence time of the tracer is independent of the Damkohler number, for the case of linear, reversible mass transfer of the 
tracer. This result indicates that if a partitioning tracer response curve could be captured completely, the correct residence time could be calculated regardless of whether local equilibrium is achieved for the tracer between the NAPL and water phases.

\subsubsection{Sensitivity Analysis}

The result from Section 3.4.2 indicates that a complete response curve will give the correct mean residence time, regardless of whether local equilibrium occurs for the tracer between the water and NAPL. However, in practice detection limits and time constraints prevent capture of a complete response curve. Sensitivity analyses using the model solution (equation (3.55)) demonstrate how mass-transfer effects impact the mean residence time calculated from a response curve. The Laplace solution was inverted numerically.

The mean residence time for a non-partitioning tracer in a column experiment should be one swept pore volume, or $t_{D}=1$. However, several more pore volumes are required to obtain enough of the response curve for an accurate result. Because of delayed breakthrough and increased tailing, partitioning tracers require more pore volumes to obtain a response curve that will give an accurate estimate of the first moment. Generally, a column test will be run until the tracer concentration drops below detection limit.

The model runs were analyzed as typical column experiments would be analyzed. The detection limit was set at three orders of magnitude below peak concentration. A maximum time limit of 15 pore volumes was set for responses where significant tailing was observed.

Figure 3.7 demonstrates the typical responses of conservative and partitioning tracers under local equilibrium conditions. The high Damkohler number (100) ensures local equilibrium is modeled. The Peclet number is set to 5, a typical laboratory value. Figure 3.8 shows tracer

response curves for $R_{f}=3.0$, and various Damkohler numbers ranging from 10 to 0.1 . Note the 
early breakthrough and pronounced tailing for the lowest Damkohler number, where limited mass transfer occurs for the tracer between the mobile water and resident NAPL phases. Figure 3.9 shows a comparison between a conservative tracer response curve and a partitioning tracer where $R_{f}=1.2$ and $N_{D a}=0.1$. Note that because of the limited mass transfer, the partitioning tracer breaks through much like the conservative tracer, but shows significant tailing at late time. Figure 3.10 is similar to Fig. 3.8, except the retardation factor is 1.2 rather than 3.0. The same effects are evident in the response curves in Fig. 3.10 as with Fig. 3.8, although the tailing is less dramatic because there is less retardation.

Table 3.2 shows the relative error in calculated retardation factors from the tracer response curves for $R_{f}=1.2$ and 3.0, at several Damkohler numbers. Note from equations (3.57) and (3.58) that theoretically, $m_{0}=1$ and $m_{1}=R_{f}$. Significant error occurs for both retardation factors at $N_{D a}=0.1$. Although Figs. 3.8 and 3.10 demonstrate mass transfer limitation effects in the response curves at $N_{D a}=1$, Table 3.2 shows that the changed shape of the response curve has little effect on the moment calculation. This result is important because it indicates that local equilibrium is not always essential to the accuracy of partitioning tracer results derived from moment theory.

Experiments detailed in Whitley (1997) showed that for gas/NAPL partitioning tracer column experiments, equilibrium conditions were achieved at a Damkohler number of approximately 5. This result agrees with the above sensitivity results, which indicate that mass transfer limitations begin to occur between $N_{D a}=10$ and $N_{D a}=1$.

The mass transfer correlation given in equation (3.13) was used to plot Damkohler number versus mean residence time for columns of various lab scale lengths, as shown in Figs. 3.11 and 3.12 for $K_{N}=30$ and 300 , respectively. The plots show that for the lower partition 
coefficient, a mean residence time of $1 \mathrm{~h}$ is adequate to achieve near equilibrium $\left(N_{D a} \sim 7\right)$ for column lengths of $3 \mathrm{~cm}$ or greater. For the higher partition coefficient, a $30 \mathrm{~cm}$ column would be recommended to achieve near equilibrium $\left(N_{D a} \sim 4\right)$ for an MRT of $1 \mathrm{~h}$.

The general rule of thumb for partitioning tracer column tests in saturated media is a 68 hour tracer mean residence time. The results shown in Figs. 3.11 and 3.12 suggest that this rule is conservative for cases where the column length is greater than $15 \mathrm{~cm}$. However, the correlation in equation (3.13) is based on a water-wet medium. Field soils may be oil-wet or mixed-wet, which would increase the residence time required to reach local equilibrium.

\subsection{Implementation of Tracer Mass Transfer in UTCHEM}

To determine the relative importance of partitioning tracer mass transfer between mobile water and immobile NAPL at the field scale, tracer mass transfer was incorporated into UTCHEM, a three-dimensional multicomponent multiphase flow and transport simulator. The general approach was to approximate tracer mass transfer between the mobile water and immobile NAPL phases by solving the two-phase, immobile case over each discrete time step $\Delta t$. A closed form analytical solution was used to calculate the change in tracer concentrations from time $t_{i}$ to time $t_{i}+\Delta t$. The following section describes the derivation of the analytical solution.

\subsubsection{Tracer Mass Transfer between Two Immobile Phases}

Consider a closed system of unit volume that contains immobile water, immobile NAPL, and some mass of partitioning tracer distributed between the two phases. Equation (3.59) describes the change in the concentration of tracer in the NAPL phase due to mass transfer of the tracer from the water phase to the NAPL phase. 


$$
\frac{f C_{N}}{f t}=\frac{K_{a w}}{\phi S_{N} K_{N}}\left(K_{N} C_{w}-C_{N}\right)
$$

Note that equation (3.59) is a rearranged form of equation (3.28).

Keeping in mind that the system has unit volume, the total mass of tracer, $M_{o}$, can be written as the sum of the mass of tracer in each of the two phases:

$$
M_{o}=C_{w} S_{w}+C_{N} S_{N}
$$

Solving equation (3.60) in terms of the tracer concentration in the water phase gives

$$
C_{w}=\frac{M_{o}-S_{N} C_{N}}{S_{w}}
$$

Substituting equation (3.61) into (3.59) eliminates the concentration of tracer in the water phase:

$$
\frac{f C_{N}}{f t}=\frac{K_{a w}}{\phi S_{N} K_{N}}\left(K_{N} \frac{M_{o}-S_{N} C_{N}}{S_{w}} y-C_{N}\right)
$$

Rearranging,

$$
\frac{f C_{N}}{f t}=\frac{K_{a w}}{\phi S_{N} K_{N}} \frac{K_{N} M_{o}}{S_{w}}-C_{N} \frac{K_{N} S_{N}}{S_{w}}+1 \sqrt[\Downarrow]{\downarrow}
$$

The definition of retardation factor (equation (3.39)) can be substituted into equation (3.63) to yield

$$
\frac{f C_{N}}{f t}=\frac{K_{a w}}{\phi S_{N} K_{N}} \frac{K_{N} M_{o}}{S_{w}}-C_{N} R_{f} \sqrt[y]{ }
$$

Applying separation of variables to equation (3.64) with the initial condition

$$
C_{N}\left(t_{i}\right)=C_{N_{O}}
$$

gives the solution in terms of the initial tracer concentration of NAPL and the total mass of tracer in the system. 


$$
C_{N}\left(t_{i}+\Delta t\right)=\frac{K_{N} M_{o}}{R_{f} S_{w}}-\frac{K_{N} M_{o}}{R_{f} S_{w}}-C_{N_{O}} \sqrt{ }_{\lrcorner} \exp \left(-R_{f} \frac{K_{a w}}{\phi S_{N} K_{N}} \Delta t\right)
$$

The concentration of the tracer in the water can then be calculated from equation (3.61).

$K_{a w}$ is calculated in UTCHEM using equation (3.13). A more detailed account of the inclusion of partitioning tracer mass transfer in the UTCHEM code can be found in Appendix B.

\subsubsection{Model Verification}

The tracer mass transfer model was tested for accuracy in two ways. First, the UTCHEM mass transfer model was shown to converge to the UTCHEM equilibrium model at high Damkohler number. Second, the UTCHEM model was compared to the 1-D analytical solution given by equation (3.55).

Figure 3.13 shows the tracer response curve for the UTCHEM equilibrium model and for the UTCHEM mass transfer model at two values of $N_{D a}$. At $N_{D a}=10$, the UTCHEM mass transfer model output is close to the equilibrium solution. The response curve for the UTCHEM mass transfer model at $N_{D a}=100$ is indistinguishable from the UTCHEM equilibrium case.

Figure 3.14 shows a comparison of the analytical solution and the UTCHEM model at $N_{D a}=0.1,1$, and 10. Good agreement is seen in all cases, with only slight divergence mostly in the tail of the response curves. The difference between the UTCHEM and analytical solutions could be the result of either a small numerical error due to the discrete approximation of the UTCHEM finite difference solution or a slight instability in the Laplace transform inversion for the analytical solution. 


\subsection{UTCHEM Simulation of Field PITT}

With the partitioning tracer mass transfer option in place and verified, UTCHEM was then used to simulate a field PITT under several different flow scenarios. The simulated field PITT setup had been previously established in support of PITTs completed at the Operational Unit 2 site at Hill AFB, Utah (Brown et. al, 1996; Brown et al., 1999; Londergan et al., 1999). The purpose of the new simulations was twofold:

1. Specifically, test the validity of the local equilibrium assumption that was made in previous simulation runs for the OU2 field PITTs.

2. More generally, evaluate the importance of mass transfer considerations for partitioning tracers at the field scale.

\subsubsection{Test Setup}

Brown et al. (1996) has a detailed description of the field site and well setup. The water table was approximately $20 \mathrm{ft}(6.1 \mathrm{~m})$ below the ground surface. A stochastic permeability field was used with a mean of 9.5 Darcies and range from $0.17 \mathrm{D}$ to $417 \mathrm{D}$. The DNAPL present at the field site was a mixture of chlorinated hydrocarbons and grease.

The well setup was a $3 \times 3$ line drive, with $10 \mathrm{ft}(3 \mathrm{~m})$ between each injection well, $10 \mathrm{ft}(3$ $\mathrm{m})$ between each extraction well, and $20 \mathrm{ft}(6.1 \mathrm{~m})$ separating the injectors from the extractors. Table 3.3 gives the partitioning tracers used in this study, along with the water/DNAPL partition coefficients.

\subsubsection{Results - 2 Day Mean Residence Time}

A simulation was first run under the conditions specified in Brown et. al (1996) for the Phase I PITT. The injection rate was $385 \mathrm{ft}^{3} / \mathrm{d}\left(1.3 \times 10^{-4} \mathrm{~m}^{3} / \mathrm{s}\right)$ for each of the three injection wells. The 
extraction rate was $481 \mathrm{ft}^{3} / \mathrm{d}\left(1.6 \times 10^{-4} \mathrm{~m}^{3} / \mathrm{s}\right)$ for each of the extraction wells. The design mean residence time for the conservative tracer was 1 day, but the mean residence time calculated from the simulation output was approximately 2 days.

Figures $3.15-3.17$ show a comparison between the equilibrium and mass transfer cases. In each figure legend, MT indicates a run with the mass transfer option enabled, while EQ indicates a run with the equilibrium option enabled. Note that the mass transfer and equilibrium cases are indistinguishable, for all of the wells. The simulations for this case demonstrated no mass transfer limitations, indicating that for this field case, the partitioning tracers were predicted to be at local equilibrium between the mobile water and immobile NAPL phases.

\subsubsection{Results - 0.1 Day Mean Residence Time}

A second simulation was run with the well rates increased by 20 times to achieve a mean residence time for the conservative tracer of approximately 0.1 day. The mean residence time for the conservative tracer calculated from the simulation output was 0.12 days. Figures 3.183.20 show the tracer output for the equilibrium and mass transfer cases. Note that again, the curves are indistinguishable on the graph. No mass transfer limitations are evident for the simulations for any of the wells, even with only a 0.1 day mean residence time.

The magnitude of the water velocity at each gridblock was output from the model, along with the calculated mass transfer coefficient. A distribution of Damkohler numbers was generated from these data for all of the partitioning tracers by assuming a path distance of $20 \mathrm{ft}$ $(6.1 \mathrm{~m})$, which is the smallest possible distance for a streamline between the wells (thus providing the most conservatively low estimate of $N_{D a}$ ). The histogram is shown in Fig. 3.21. Note that all of the values are above 10 and the logarithmic mean is approximately 500 . Given 
this distribution of values, near equilibrium partitioning would be expected, as was shown in the tracer concentration response data.

The simulations indicate that a residence time of only a few hours is required to achieve local equilibrium for a field scale PITT. However, the simulations were made for only a small range of possible field parameters. This result cannot be extended to all PITTs under all conditions. Other scenarios with different assumptions, several of which are given below, could change the answer significantly.

1. The mass transfer coefficient correlation (equation (3.13) may not be valid at high flow rates, although the velocities in the 0.1 day MRT simulation were within the bounds suggested by Miller et al. (1990).

2. The mass transfer coefficient correlation was derived from column data for strongly water-wet media. The correlation would not be as accurate for the mixed-wet or oil-wet conditions that exist at many NAPL contaminated sites, because the NAPL is less accessible to the mobile water phase (and to the dissolved tracer).

3. The analysis of the relative importance of the NAPL phase resistance (section 3.2.2) was based on several simplifying assumptions. The NAPL phase resistance may be more important at higher flow rates or under different wettabilities.

Experiments and investigations that could help evaluate these possibilities are proposed in Section 7.

\subsection{Summary and Conclusions}

The concept of mass transfer was explained in terms of NAPL/water partitioning tracers. Tworesistance theory was used as the fundamental basis for the development of a 1-D partitioning tracer transport model that included mass transfer. 
An aqueous phase mass transfer coefficient correlation that was originally developed to describe NAPL dissolution was applied to tracers partitioning from water into NAPLs. A simple NAPL phase mass transfer coefficient was developed based on the assumption of spherical NAPL blobs in the porous media. A deterministic sensitivity analysis indicated that the NAPL phase resistance would be negligible for a range of input parameters typical for PITTs in porous media. The Damkohler number determined using this correlation was either independent of or very weakly dependent on the NAPL saturation, depending on the reported value of the exponent.

The 1-D model was analyzed to determine the theoretical mean residence time of a partitioning tracer with mass transfer. The mean residence time was determined to be equal to the equilibrium case, given complete capture of the tracer response curve. The 1-D model was used to determine how mass transfer limitations affected the NAPL volume estimate given more realistic time and measurement constraints. Mass transfer had a visual impact on the partitioning tracer response curves at Damkohler numbers of approximately 10 or less. Mass transfer had an impact on moment analysis results at Damkohler numbers of approximately 1 or less.

The partitioning tracer mass transfer correlation was implemented in UTCHEM, at 3-D transport simulator. Field scale simulations indicated that even at very small mean residence times, i.e., 0.1 day, mass transfer limitations did not affect the partitioning tracer concentration response curves. This result is dependent on the validity of the water phase mass transfer correlation, and on the assumption of negligible contribution by the NAPL phase resistance. 


\subsection{Tables}

Table 3.1. Input Values for Sensitivity Study of Overall Phase Resistance

\begin{tabular}{|crrrc|}
\hline variable & base value & \multicolumn{1}{c}{ min } & max & units \\
\hline \hline$\theta_{N}$ & 0.03 & 0.01 & 0.1 & \\
$u_{w}$ & $5 . \mathrm{E}-03$ & $5 . \mathrm{E}-04$ & $5 . \mathrm{E}-02$ & $\mathrm{~cm} / \mathrm{s}$ \\
$d_{N}$ & 0.01 & 0.001 & 0.1 & $\mathrm{~cm}$ \\
$d_{50}$ & 0.1 & 0.001 & 0.1 & $\mathrm{~cm}$ \\
$\rho_{w}$ & 1 & & & $\mathrm{~g} / \mathrm{cm}^{3}$ \\
$\mu_{w}$ & 0.01 & & & $\mathrm{~g} / \mathrm{cm}^{2} \mathrm{~s}$ \\
$D_{w}$ & $1 . \mathrm{E}-05$ & & & $\mathrm{~cm}^{2} / \mathrm{s}$ \\
$K_{N}$ & 30 & & & \\
$D_{N}$ & $1.5 \mathrm{E}-05$ & & & $\mathrm{~cm}^{2} / \mathrm{s}$ \\
\hline
\end{tabular}

Table 3.2: Summary of Results for Sensitivity Analysis of 1-D Model

\begin{tabular}{|rrrrrr|}
\hline $\mathbf{R}_{\mathbf{f}}$ & \multicolumn{1}{c}{$\mathbf{N}_{\mathbf{D a}}$} & \multicolumn{1}{c}{$\mathbf{m}_{\mathbf{0}}$} & \%error & $\mathbf{m}_{\mathbf{1}}$ & \%error \\
0 & 100 & 1.00 & 0.06 & 1.00 & 0.29 \\
1.2 & 100 & 1.00 & 0.06 & 1.20 & 0.28 \\
1.2 & 10 & 1.00 & 0.06 & 1.20 & 0.33 \\
1.2 & 1 & 1.00 & 0.11 & 1.19 & 0.68 \\
1.2 & 0.1 & 0.99 & 1.02 & 1.02 & 17.16 \\
3 & 100 & 1.00 & 0.13 & 2.98 & 0.51 \\
3 & 10 & 1.00 & 0.07 & 2.99 & 0.37 \\
3 & 1 & 1.00 & 0.29 & 2.96 & 1.50 \\
3 & 0.1 & 0.95 & 5.03 & 1.76 & 70.60 \\
\hline
\end{tabular}


Table 3.3: Partitioning Tracers Used in OU2 Simulations

\begin{tabular}{|l|c|}
\hline \multicolumn{1}{|c|}{ Tracer } & $\begin{array}{c}K_{N} \\
(\mathrm{mg} / \mathrm{L}) /(\mathrm{mg} / \mathrm{L})\end{array}$ \\
\hline \hline 1-propanol & 0.0 \\
\hline 1-pentanol & 3.87 \\
\hline 2-ethyl-1-butanol & 12.5 \\
\hline 1-heptanol & 140.5 \\
\hline
\end{tabular}

\subsection{Figures}

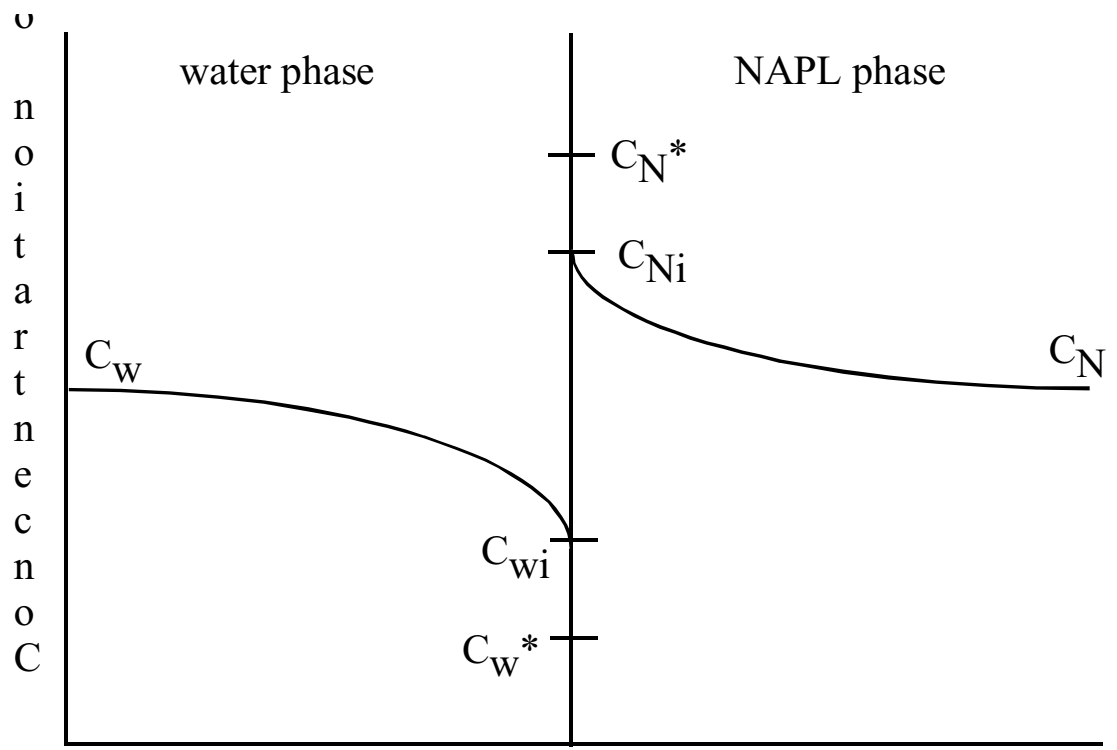

Distance through the phases

Figure 3.1: Illustration of interphase solute concentration. 


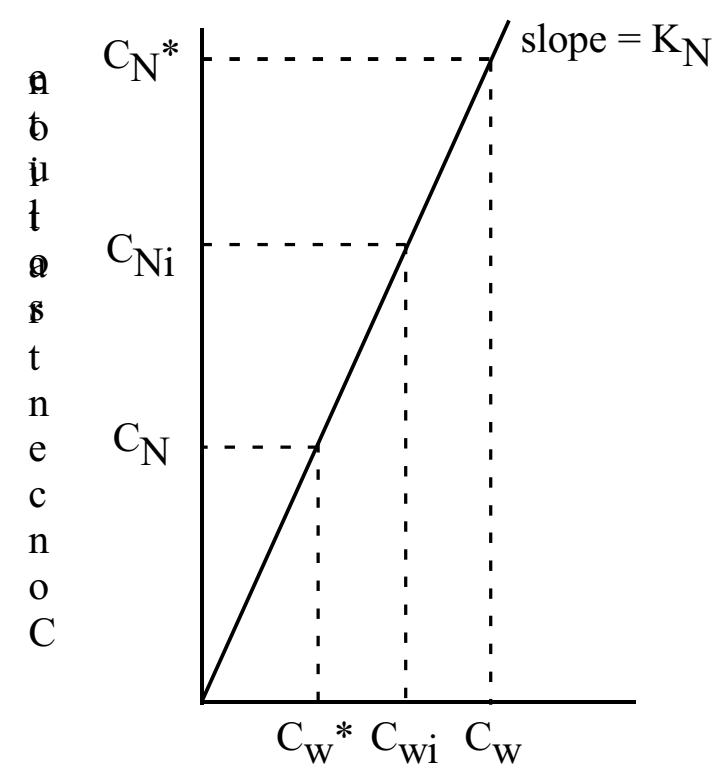

Concentration of solute in water

Figure 3.2: Illustration of solute distribution between water and NAPL.

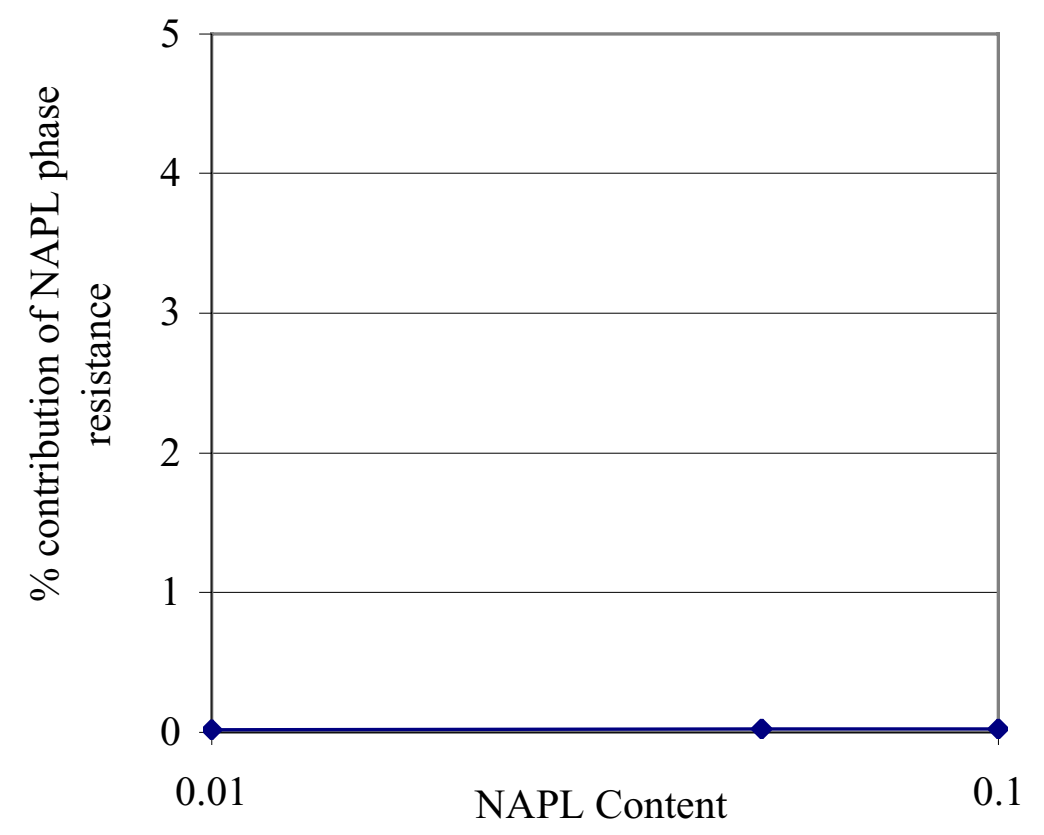

Figure 3.3: Percent NAPL phase resistance with varying $\theta_{N}$. 


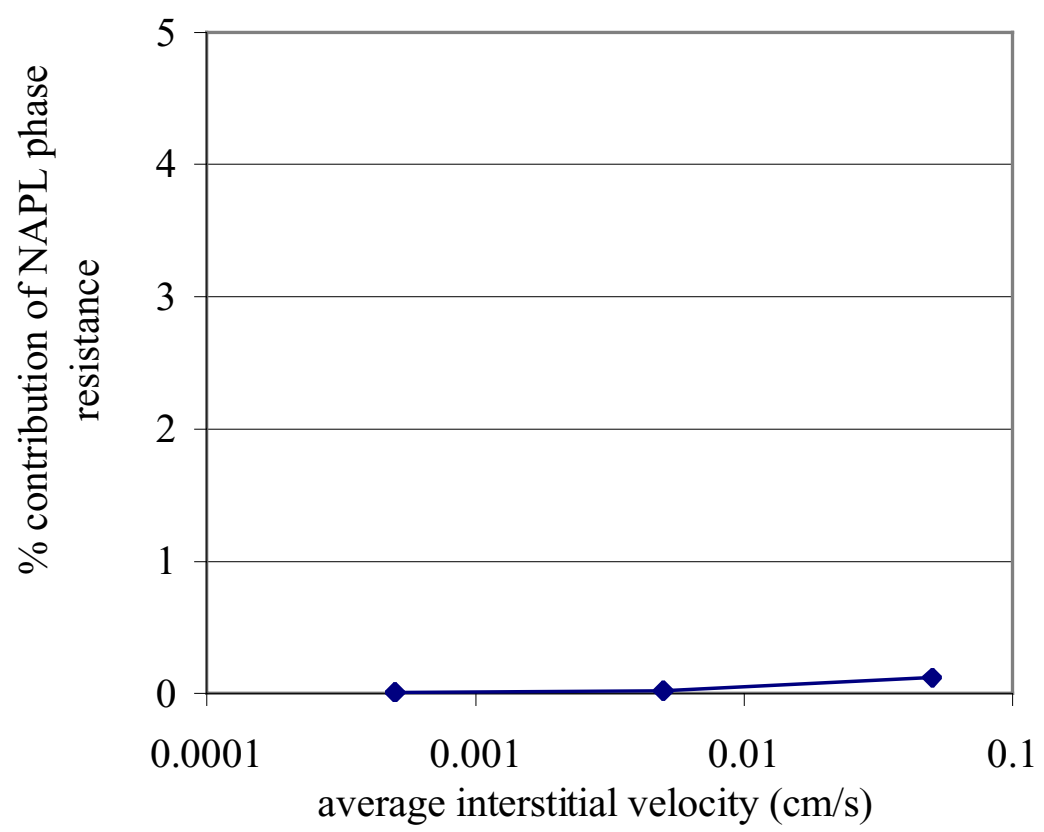

Figure 3.4: Percent NAPL phase contribution with varying $u$.

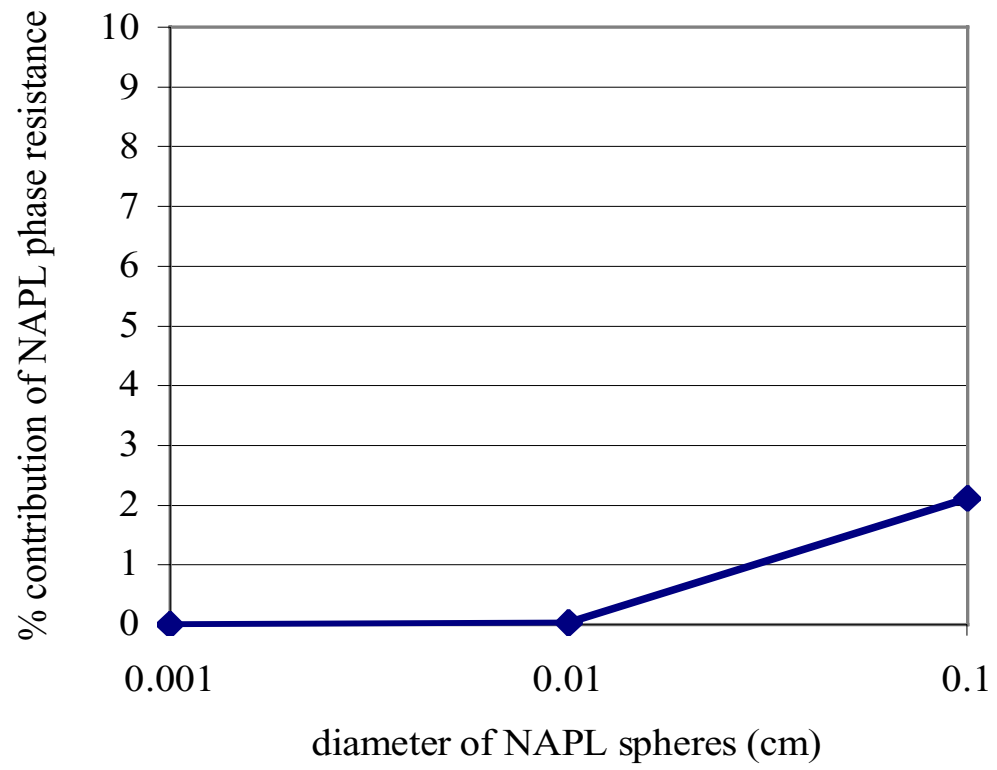

Figure 3.5: Percent NAPL phase contribution with varying $d_{N}$. 


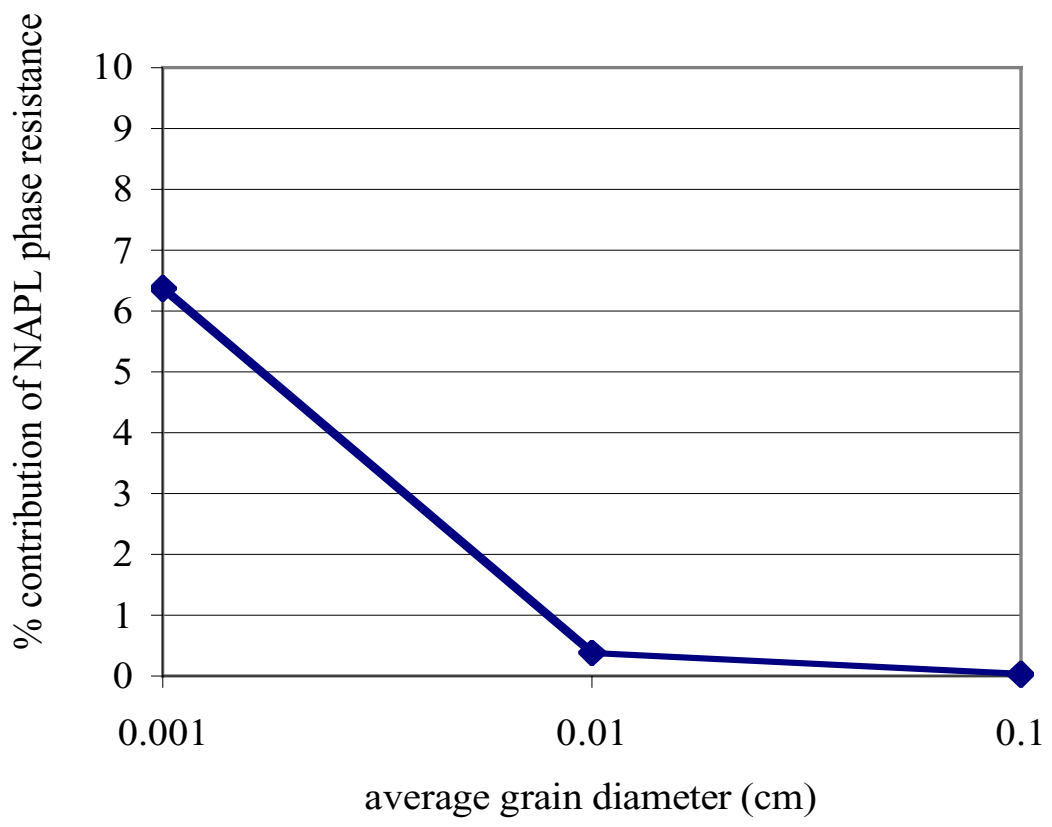

Figure 3.6: Percent NAPL phase contribution with varying $d_{50}$.

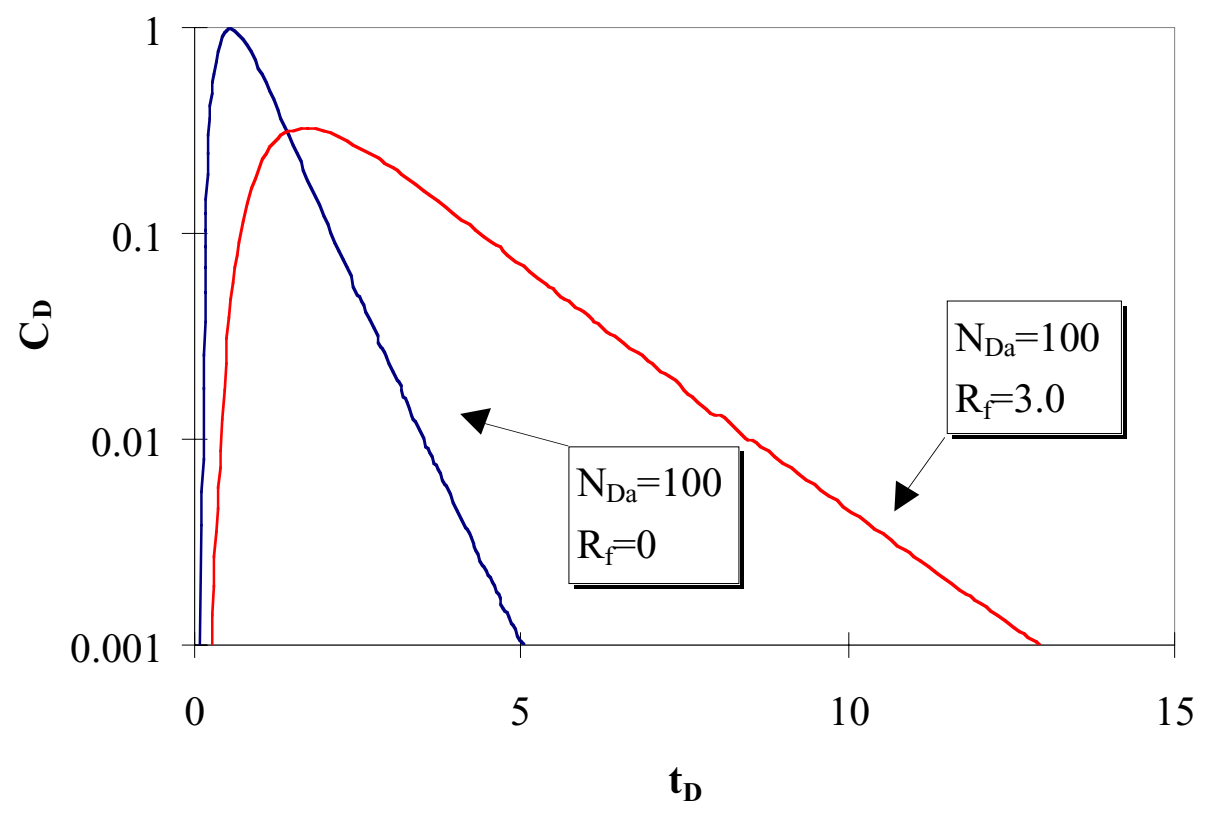

Figure 3.7: Tracer responses under local equilibrium conditions. 


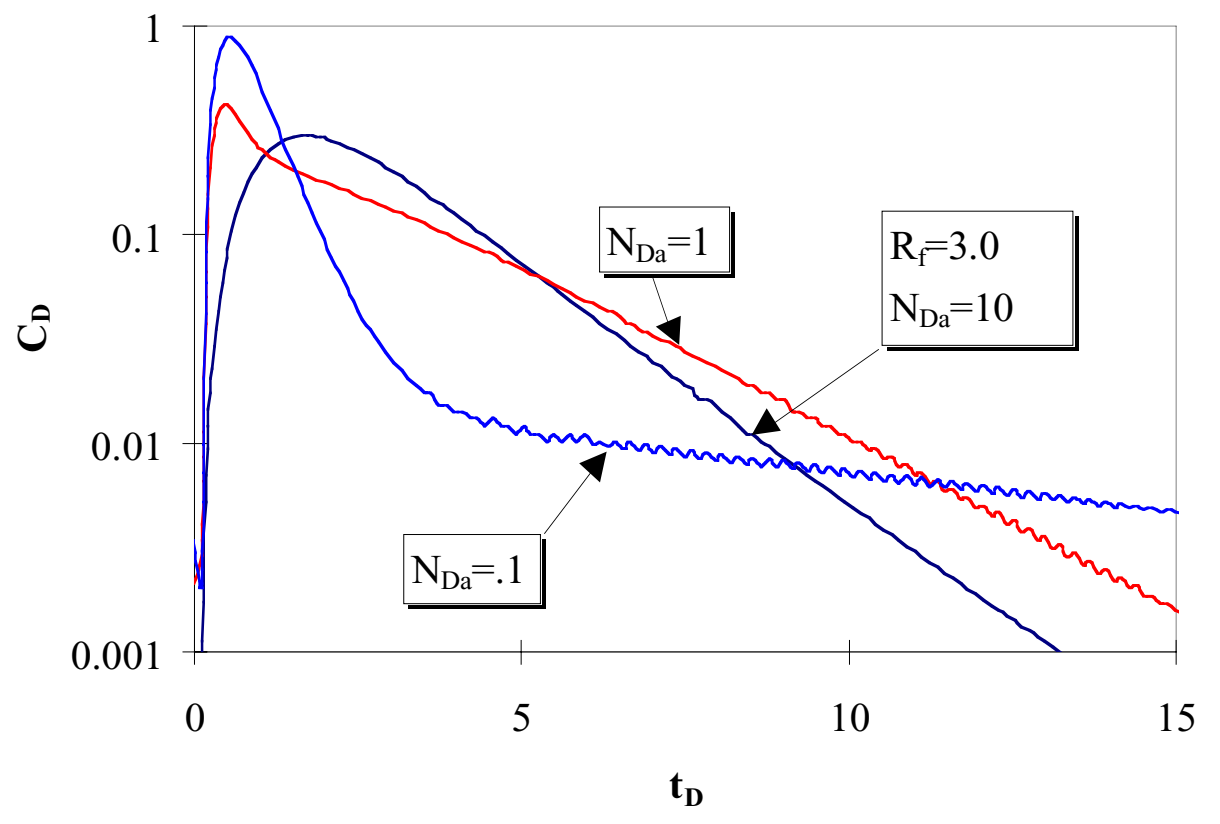

Figure 3.8: Partitioning tracer responses for varying Damkohler numbers.

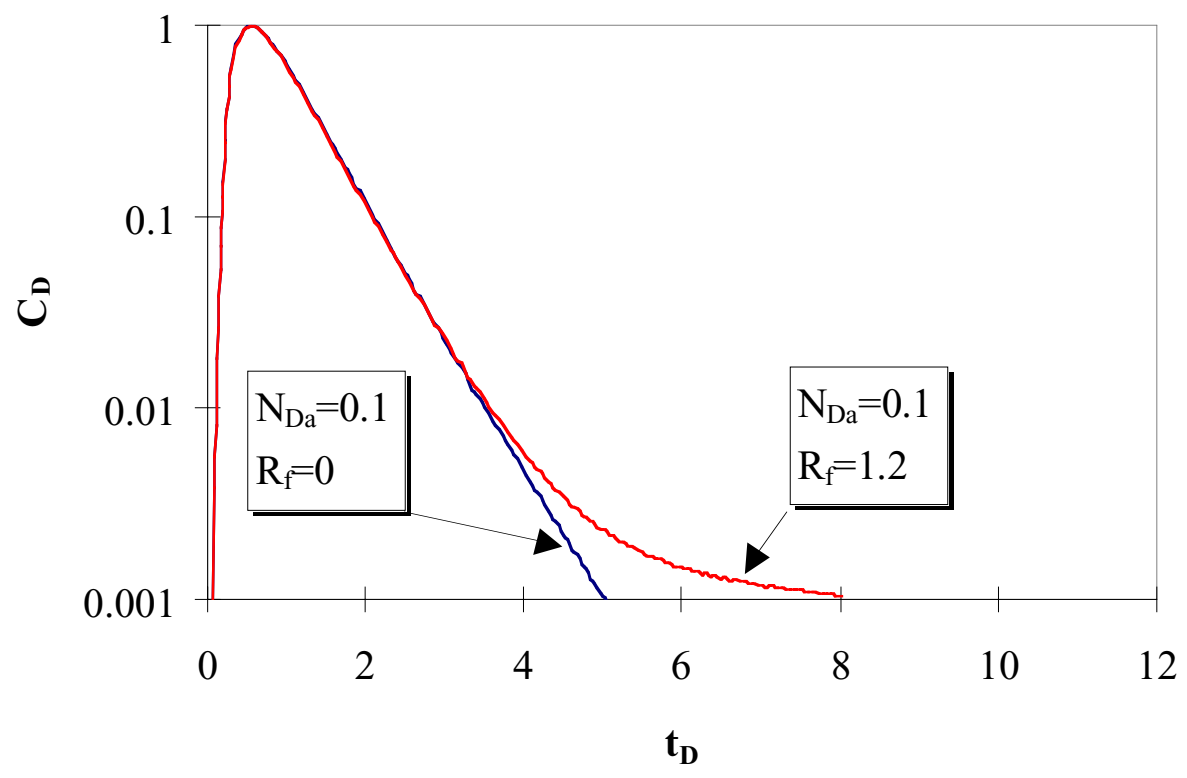

Figure 3.9: Tracer responses under limited mass transfer conditions. 


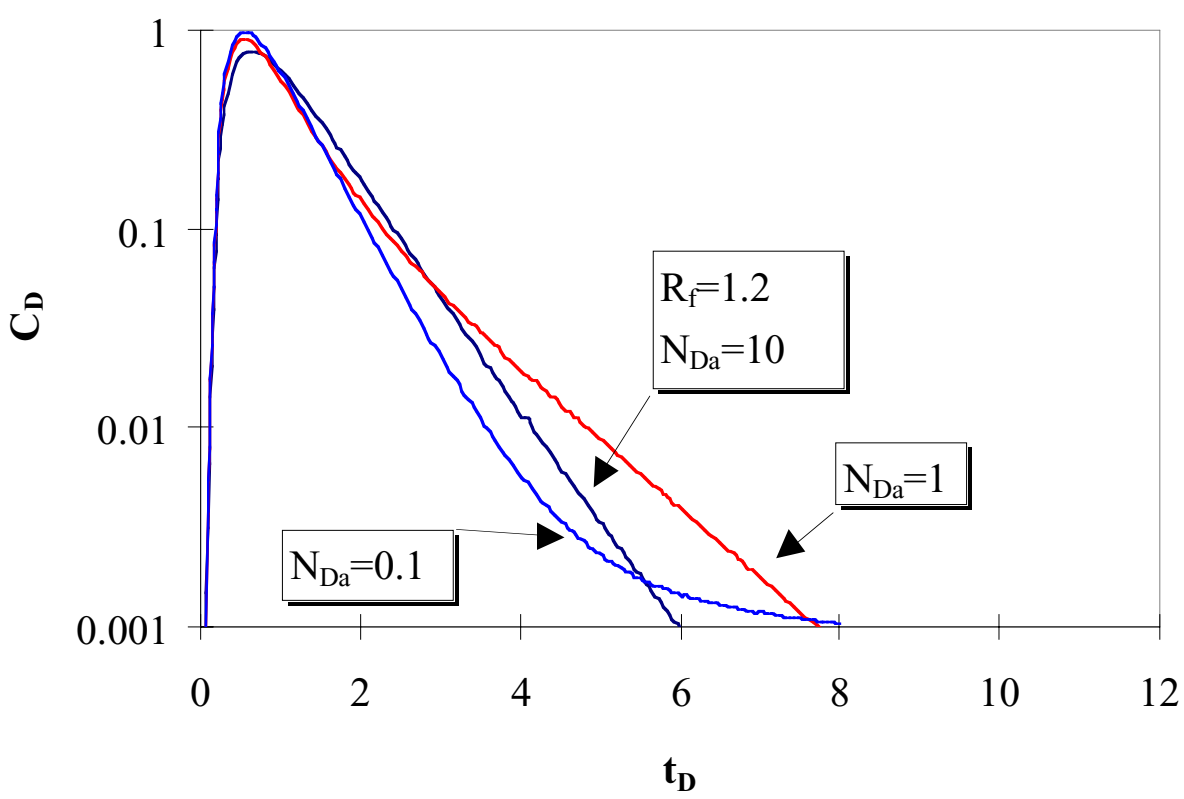

Figure 3.10: Partitioning tracer responses for varying Damkohler numbers.

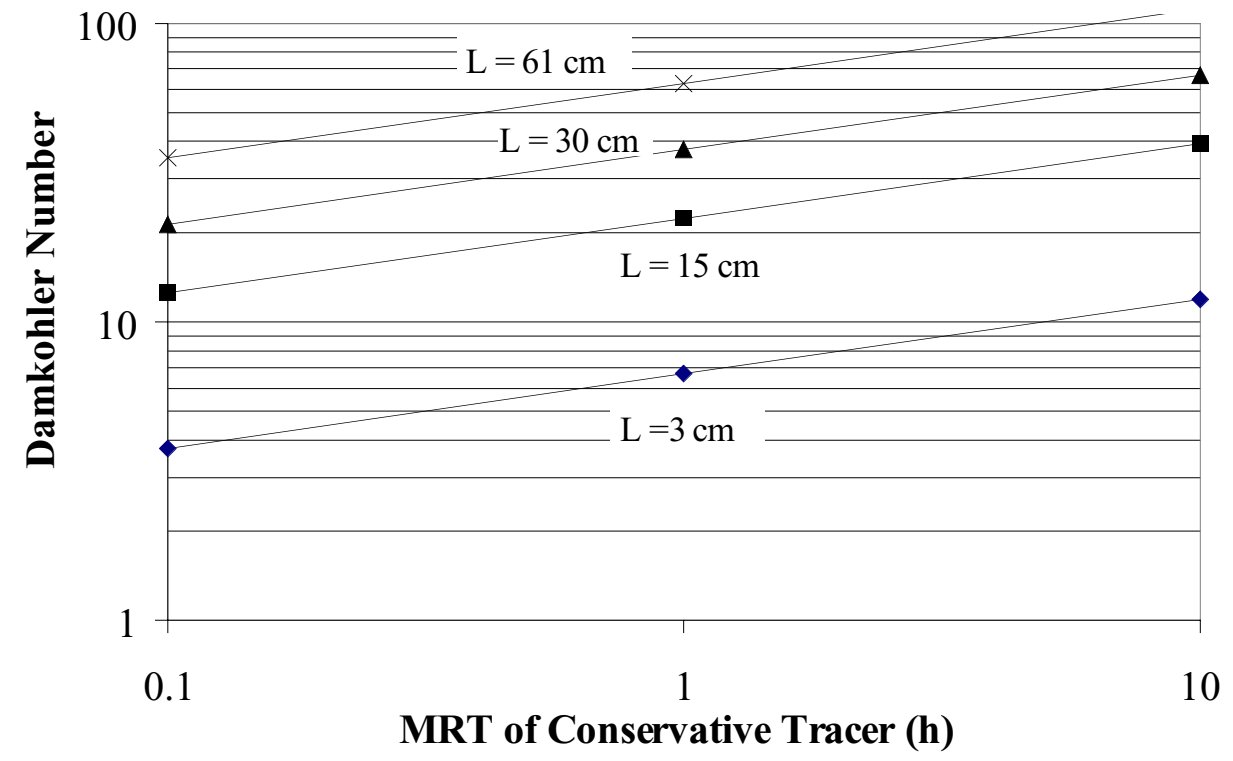

Figure 3.11: $N_{D a}$ vs. MRT for various column lengths, $K_{N}=30$. 


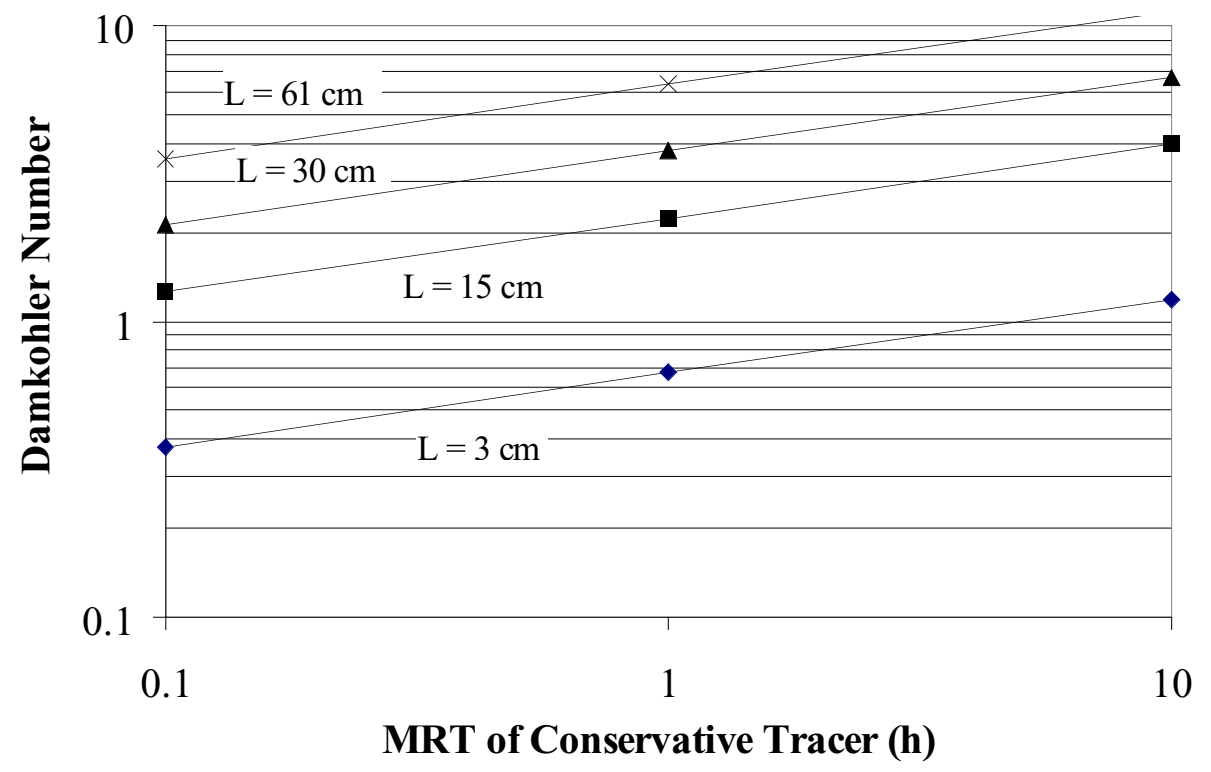

Figure 3.12: $N_{D a}$ vs. MRT for various column lengths, $K_{N}=300$.

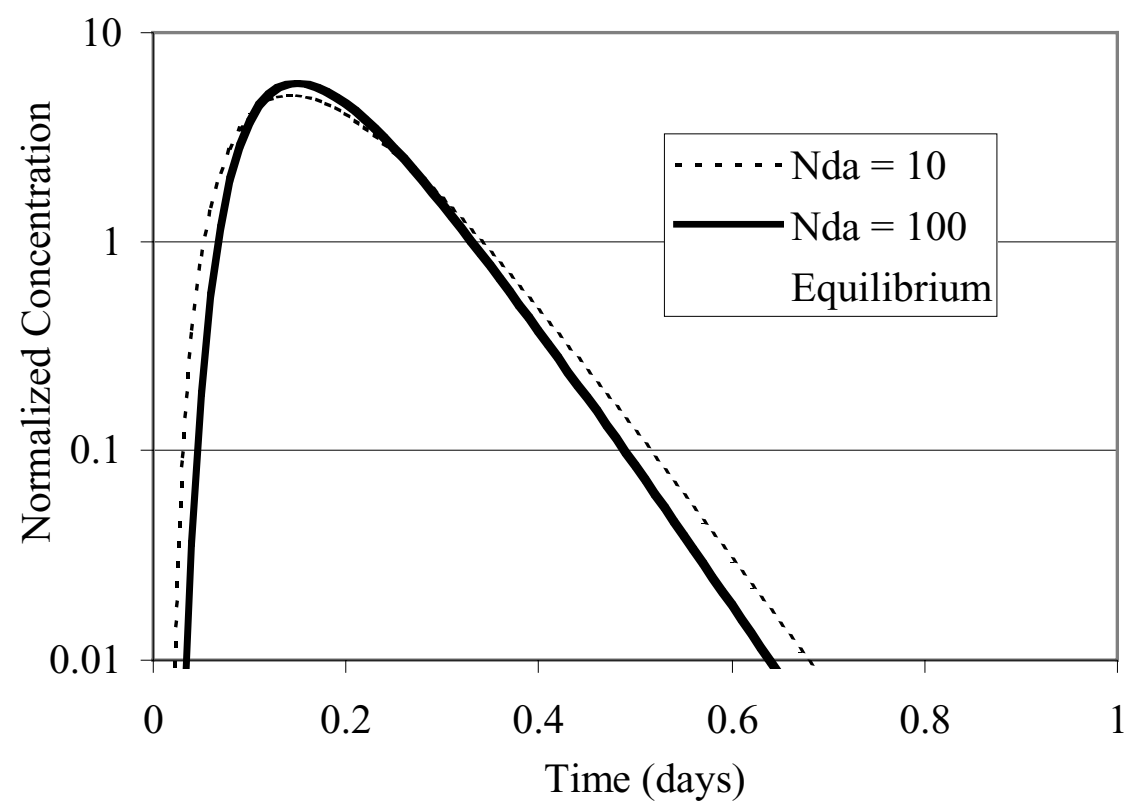

Figure 3.13: Comparison of UTCHEM equilibrium and mass transfer models. 


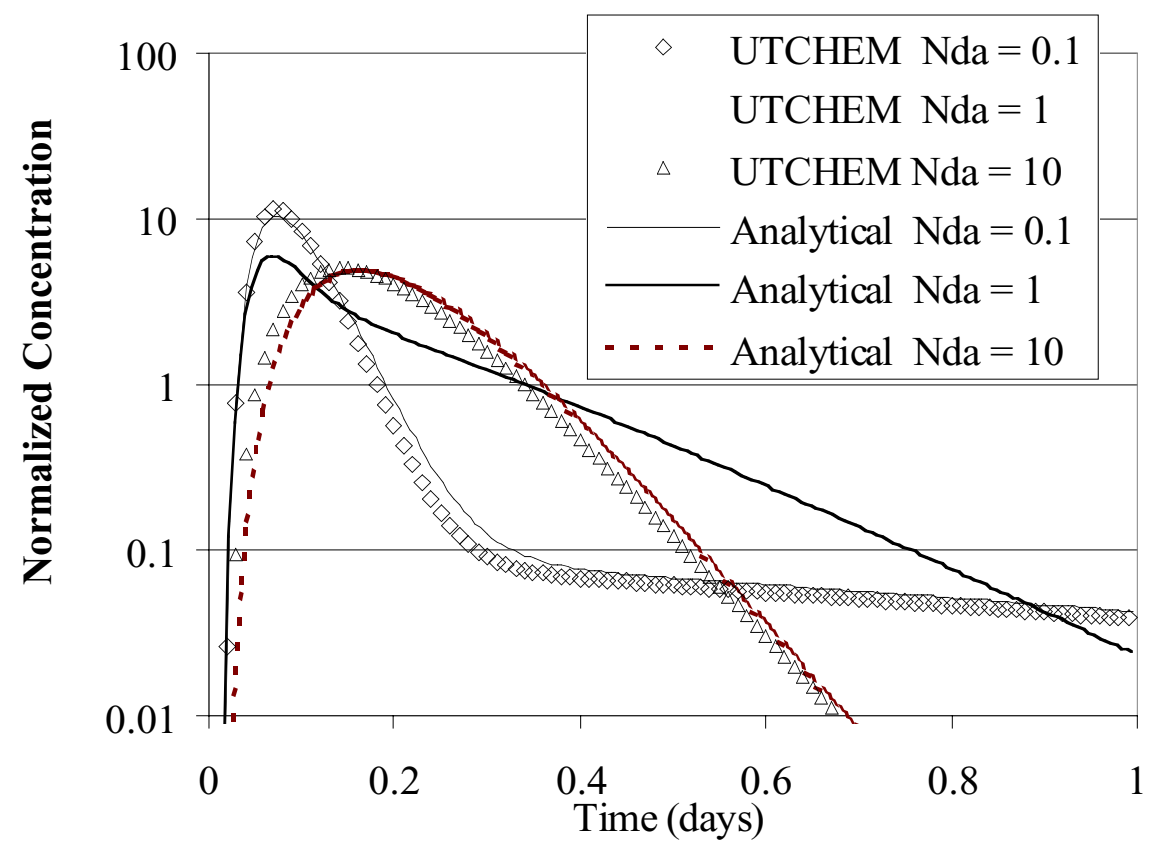

Figure 3.14: Comparison of UTCHEM and analytical tracer mass transfer. 

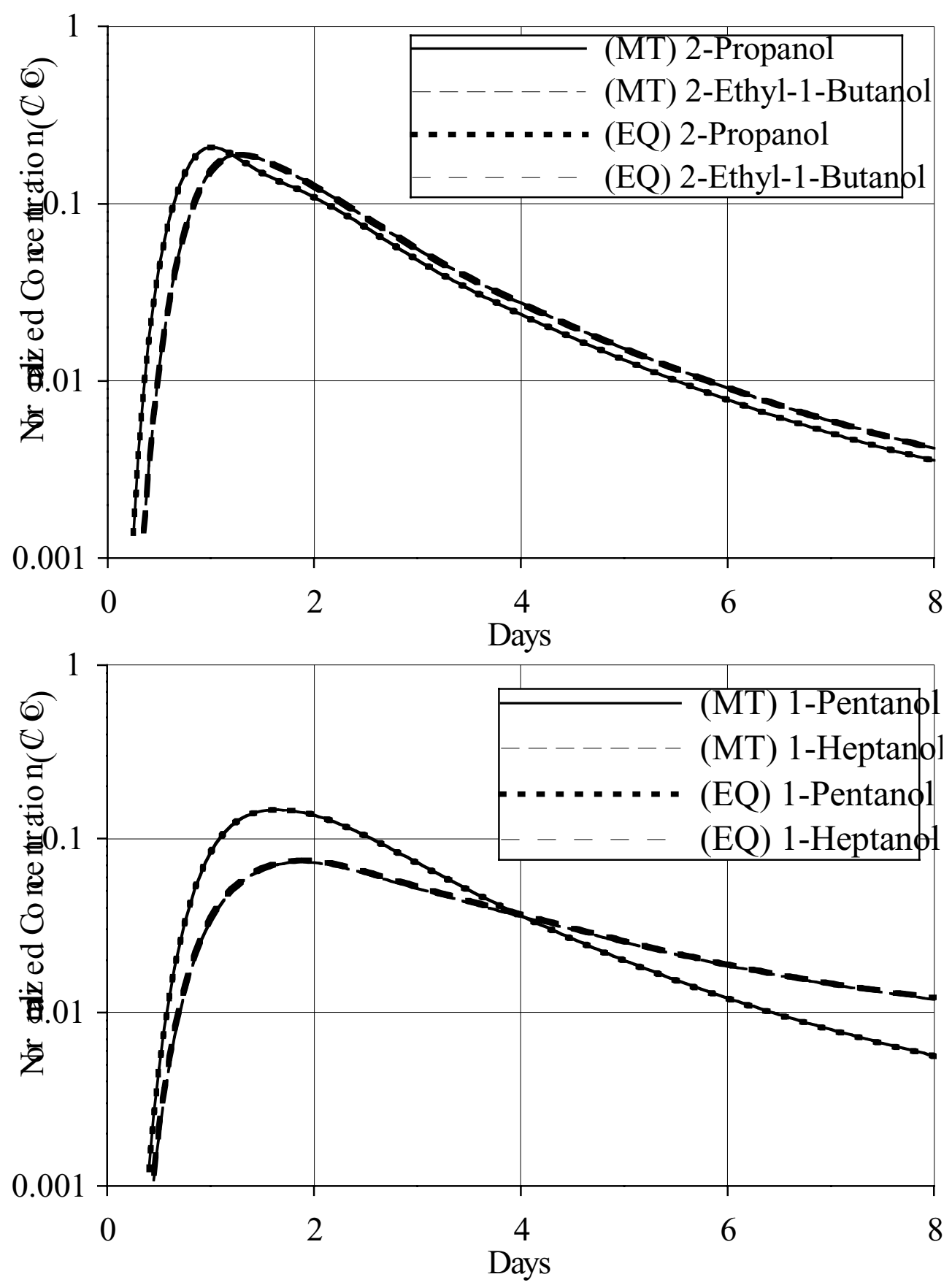

Figure 3.15: Well U21 response curves, UTCHEM OU2 field PITT simulation using mass transfer (MT) and equilibrium (EQ) options, 2 day MRT. 

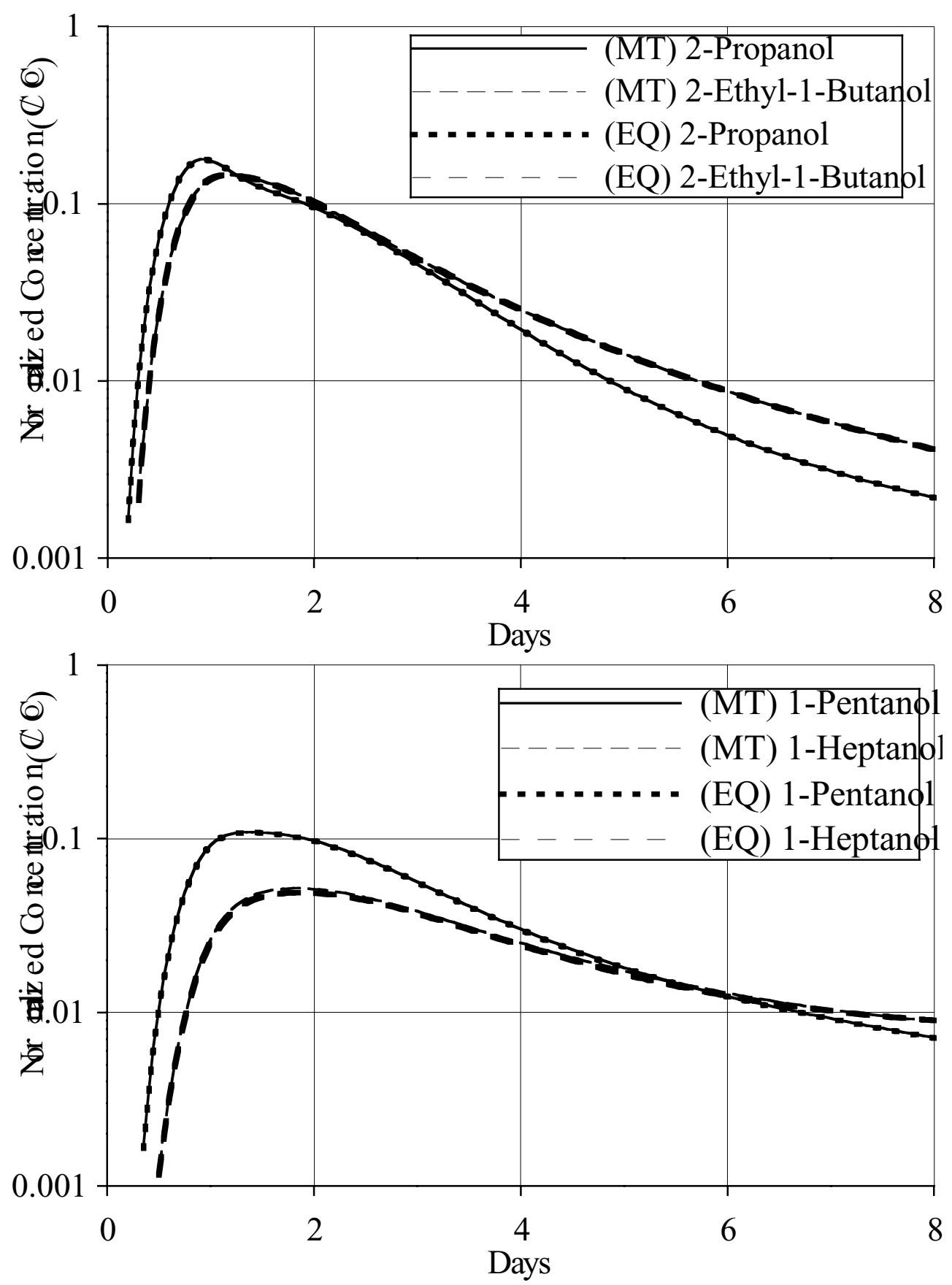

Figure 3.16: Well SB1 response curves, UTCHEM OU2 field PITT simulation using mass transfer (MT) and equilibrium (EQ) options, 2 day MRT. 

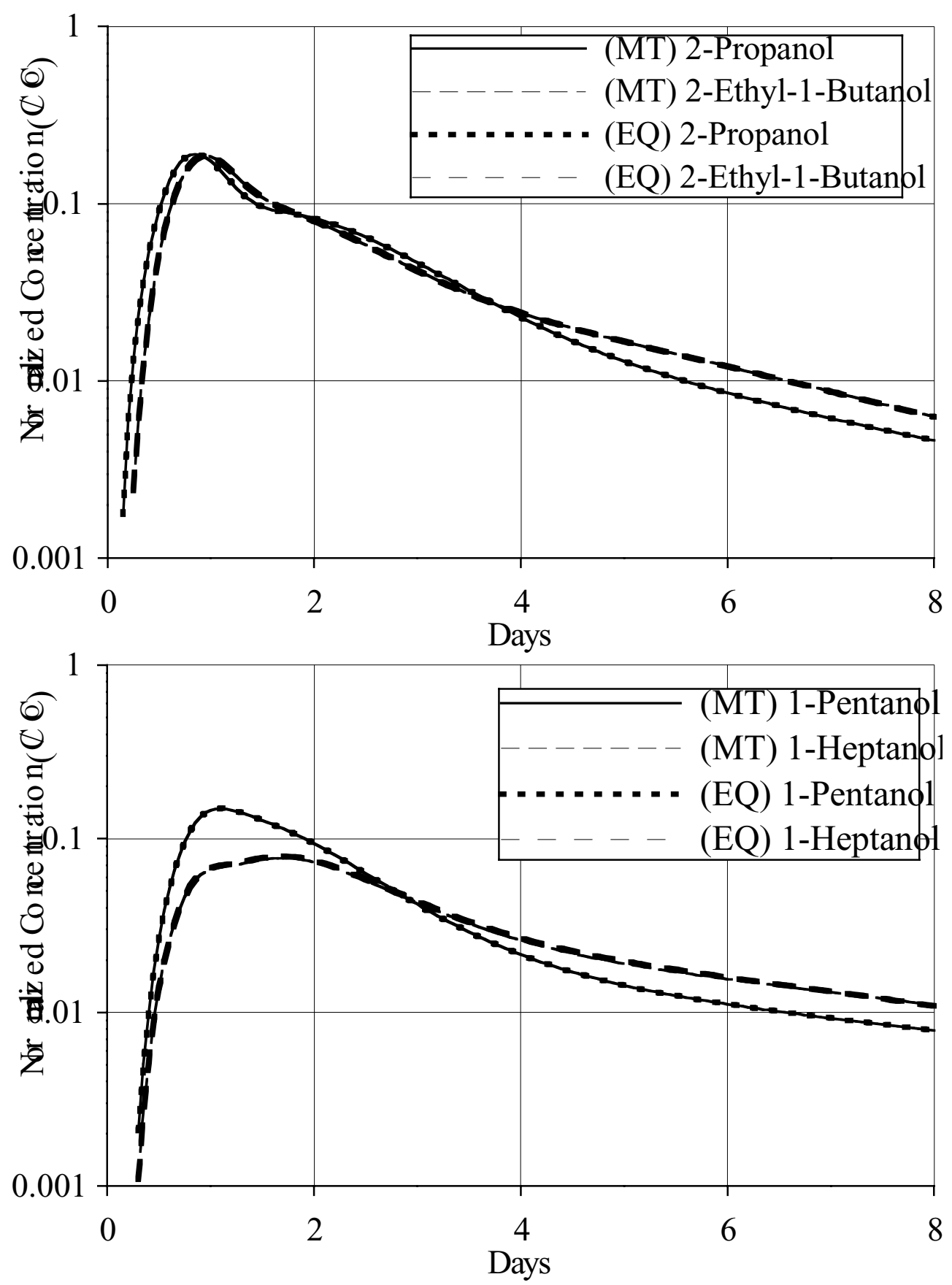

Figure 3.17: Well SB5 response curves, UTCHEM OU2 field PITT simulation using mass transfer (MT) and equilibrium (EQ) options, 2 day MRT. 

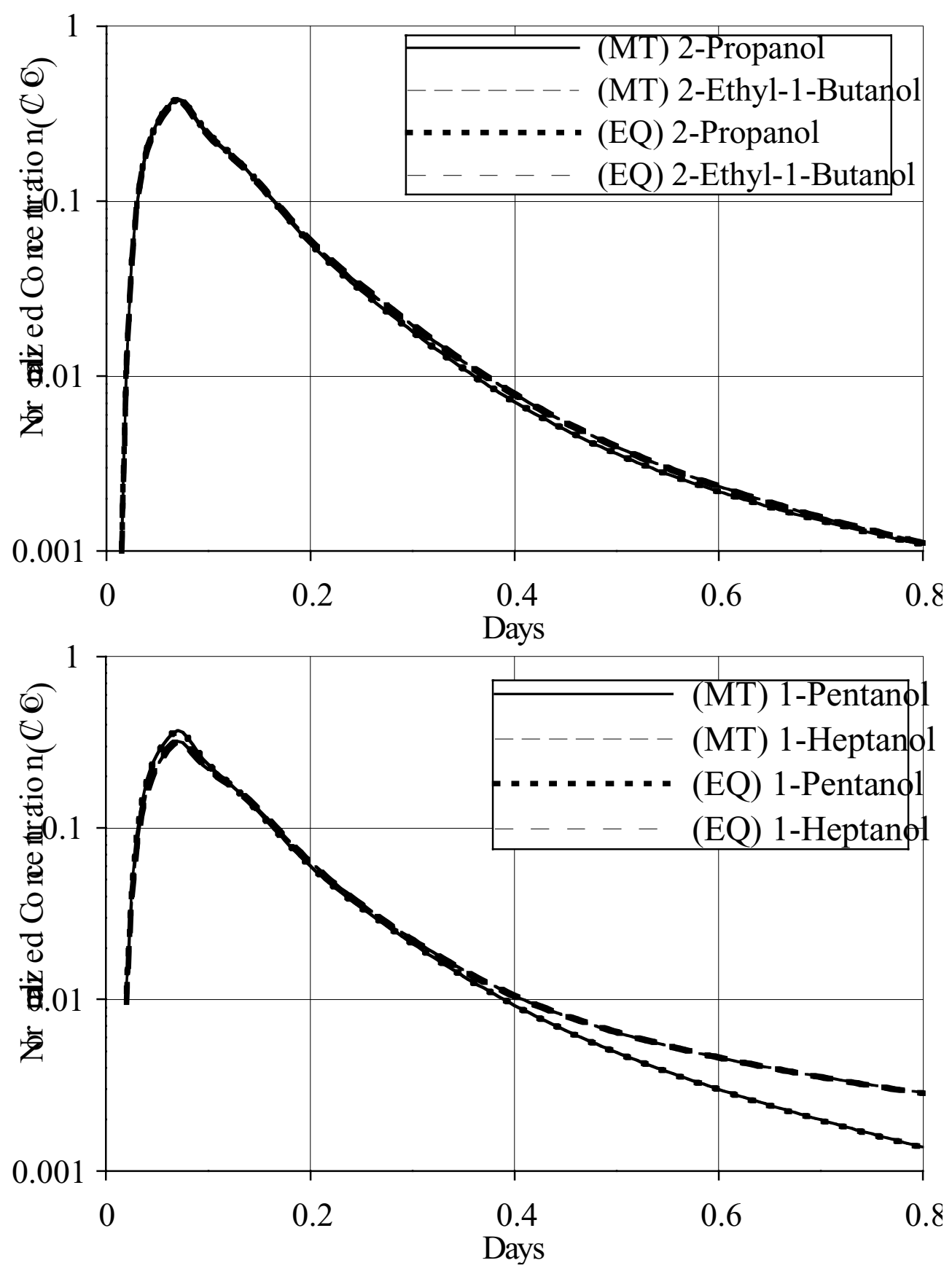

Figure 3.18: Well U21 response curves, UTCHEM OU2 field PITT simulation using mass transfer (MT) and equilibrium (EQ) options, 0.1 day MRT. 

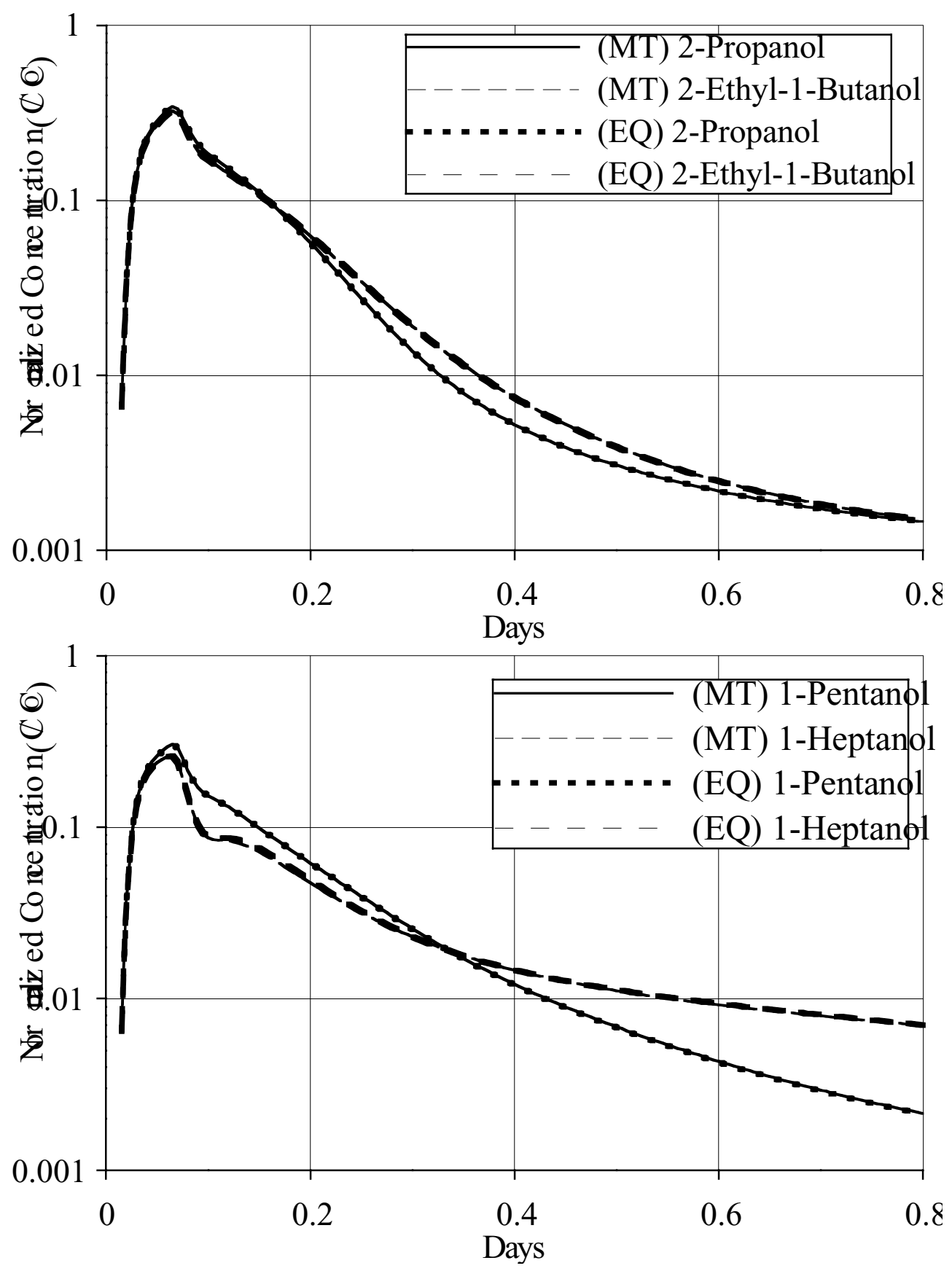

Figure 3.19: Well SB1 response curves, UTCHEM OU2 field PITT simulation using mass transfer (MT) and equilibrium (EQ) options, 0.1 day MRT. 

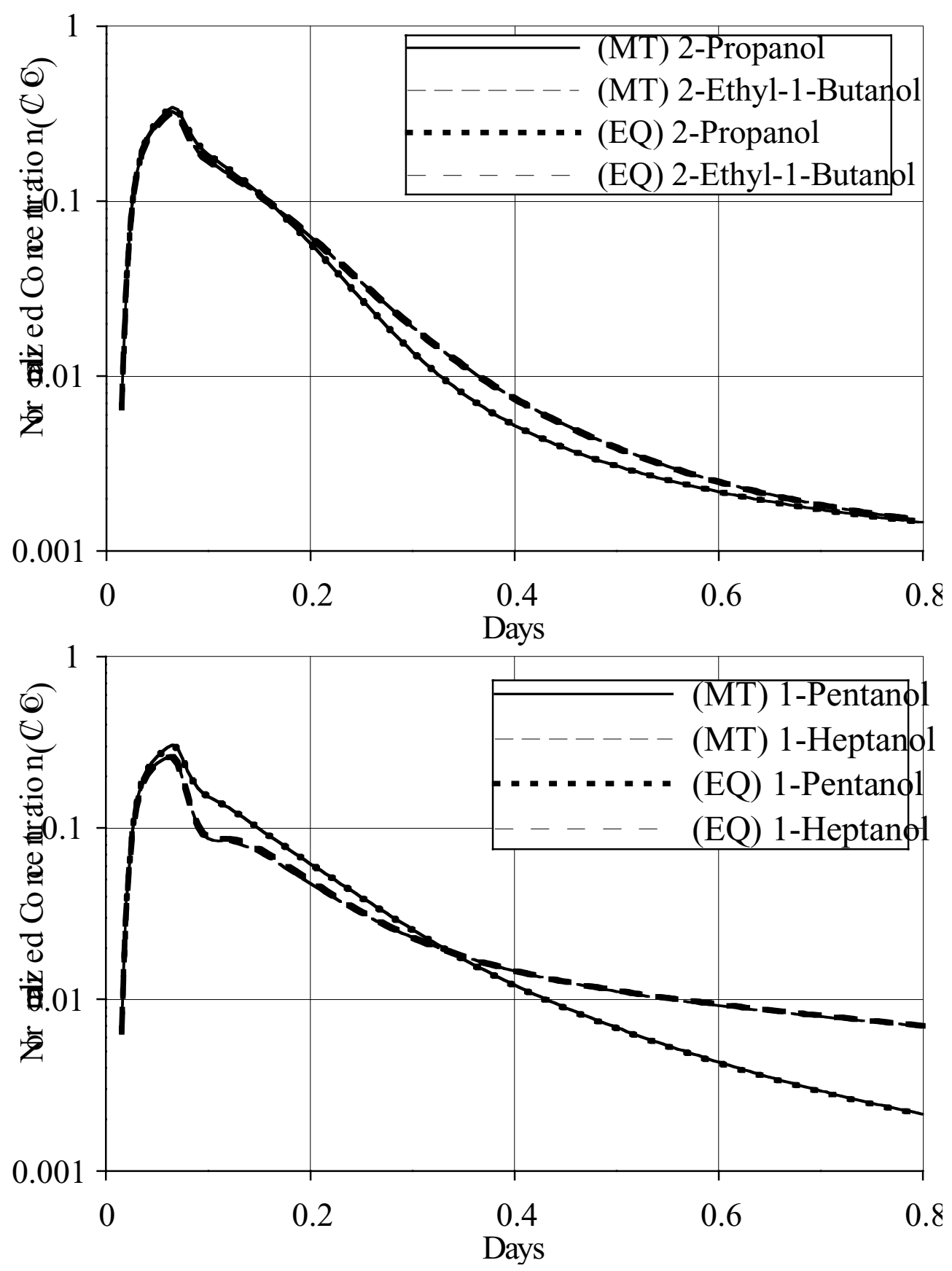

Figure 3.20: Well SB5 response curves, UTCHEM OU2 field PITT simulation using mass transfer (MT) and equilibrium (EQ) options, 0.1 day MRT. 


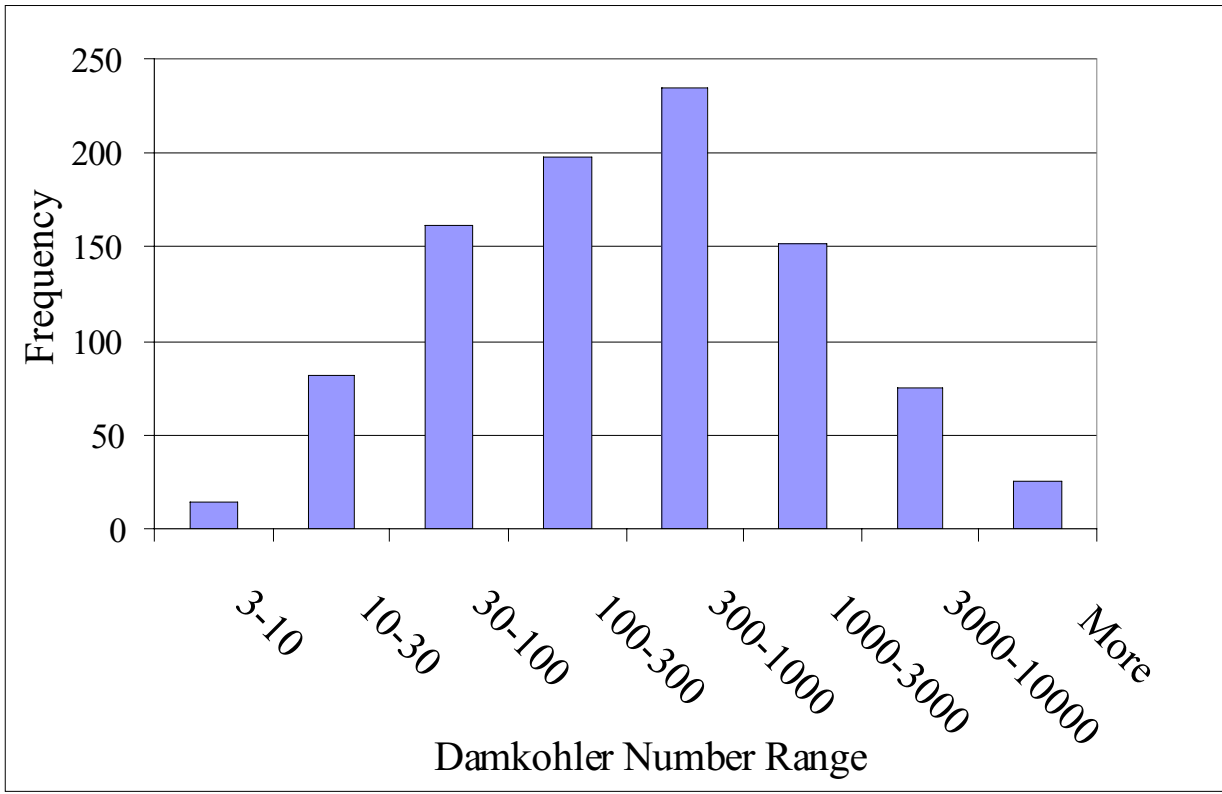

Figure 3.21: Damkohler number distribution for 0.1 day MRT simulation. 



\section{Section 4: Partitioning Tracer Transport in Fractured Media}

\subsection{Partitioning Tracers in Fractured Media with Mass Transfer}

In porous media, local equilibrium can be achieved for the tracer between the water and NAPL phases in most cases simply by controlling the flow rate of the mobile water phase. In fractured media, however, lower flow rates will increase matrix diffusion effects, which may compromise the result. The following describes development of a model that combines the effects of first order, reversible tracer mass transfer between the water and NAPL phases with tracer diffusion into a surrounding rock matrix. The model development is followed by a sensitivity analysis of the parameter groups unique to the fracture transport.

\subsubsection{Determination of Relevant Parameters}

The following development combines the model of equations (3.21) and (3.28) with a parallel fracture model similar to that of Becker (1996). The model describes 1-D tracer fracture transport in the $\mathrm{x}$-direction, with lumped diffusion/dispersion in the fracture, reversible first order tracer mass transfer between the water and NAPL phases in the fracture, and orthogonal diffusive tracer transport between the fracture and surrounding matrix. Figure 4.1 illustrates the parallel fracture concept, where the fracture spacing is assumed to be constant. The advantage of the parallel fracture model over single fracture models is that the parallel fracture model yields a response curve with a finite first moment. Single fracture models such as Tang et al. (1981) assume a matrix of infinite extent, which yields a correspondingly infinite first moment for the response curve (Neretnieks, 1983; Becker, 1996). 
The equation for flow in the fracture is similar to equation (3.21) where the same assumptions have been made and a loss term $q$ has been added to account for diffusion of the tracer from the fracture to the matrix

$$
\frac{f C_{w}}{f t}+\frac{S_{N}}{S_{w}} \frac{f C_{N}}{f t}+u \frac{f C_{w}}{f x}-D_{L} \frac{f^{2} C_{w}}{f x^{2}}-q=0
$$

The loss term is described by Fick s first law with the concentration gradient normal to the matrix surface, written as

$$
\begin{aligned}
& q=\left.\frac{D_{m} 2 \phi_{m}}{\phi_{f} S_{w} L} \frac{f C_{m}}{f y}\right|_{y=b} \\
& C_{m}=\text { tracer concentration in the matrix } \\
& 2 b=\text { aperture width } \\
& L=\text { fracture spacing (see Fig. } 4.1 \text { ) } \\
& D_{m}=\text { diffusion coefficient for the tracer in the matrix } \\
& \phi_{m}=\text { matrix porosity }
\end{aligned}
$$

Note that $\frac{2 \phi_{m}}{L}$ is the fracture/matrix contact area per unit fracture volume. The fracture porosity can be written in terms of the aperture and fracture spacing so that equation (4.2) becomes

$$
q=\frac{1}{\phi_{f}} \sqrt{ } \frac{D_{m} 2 \phi_{m}}{S_{w} L} \frac{f C_{m}}{f y}=\frac{L}{2 b} \sqrt{ } \frac{D_{m} 2 \phi_{m}}{S_{w} L} \frac{f C_{m}}{f y}=\frac{D_{m} \phi_{m}}{S_{w} b} \frac{f C_{m}}{f y}
$$

with $b$ representing half the aperture width (Fig. 4.1). Equations (4.1) and (4.3) are combined to yield

$$
\frac{f C_{w}}{f t}+\frac{S_{N}}{S_{w}} \frac{f C_{N}}{f t}+u \frac{f C_{w}}{f x}-D_{L} \frac{f^{2} C_{w}}{f x^{2}}-\left.\frac{\phi_{m} D_{m}}{S_{w} b} \frac{f C_{m}}{f y}\right|_{y=b}=0
$$

The equation for the changing concentration in the NAPL phase is identical to equation (3.28) 


$$
\frac{f C_{N}}{f t}=\frac{K_{a w}}{\phi_{f} S_{N}}\left(C_{w}-\frac{C_{N}}{K_{N}}\right)
$$

and the diffusion in the matrix is described by

$$
\frac{f C_{m}}{f t}=D_{m} \frac{f^{2} C_{m}}{f y^{2}}
$$

Using the same technique as described in section 3.4.1, inspectional analysis transforms equations (4.4) to (4.6) to dimensionless form:

$$
\begin{aligned}
& \frac{f C_{D, w}}{f t_{D}}+\left(R_{f}-1\right) \frac{f C_{D, N}}{f t_{D}}+\frac{f C_{D, w}}{f x_{D}}-\frac{1}{N_{P e}} \frac{f^{2} C_{D, w}}{f x_{D}^{2}} \\
& -\left.\frac{\phi_{m}}{\phi_{f} S_{w}} N_{M a} \frac{f C_{D, m}}{f y_{D}}\right|_{y_{D}=\phi_{f}}=0 \\
& \frac{f C_{D, N}}{f t_{D}}=N_{D a}\left(C_{D, w}-C_{D, N}\right) \\
& \frac{f C_{D, m}}{f t_{D}}=N_{M a} \frac{f^{2} C_{D, m}}{f y_{D}^{2}}
\end{aligned}
$$

The additional dimensionless variable $y_{D}$ is defined as follows:

$$
y_{D}=\frac{2 y}{L}
$$

The dimensionless parameters are

$$
\begin{aligned}
& R_{f}=1+\frac{K_{N} S_{N}}{S_{w}} \\
& N_{P e}=\frac{u x_{L}}{D_{L}} \\
& N_{D a}=-\frac{K_{a w}}{K_{N} \phi_{f} S_{N}} \sqrt{\frac{x_{L}}{u} \sqrt{ }}
\end{aligned}
$$




$$
N_{M a}=\frac{4 D_{m}}{L^{2}} \frac{x_{L}}{u}
$$

The first three dimensionless parameters, retardation factor, Peclet number, and Damkohler number respectively, are the same as those in the model of Section 3.4.1. The new parameter, $N_{M a}$, is the ratio of two characteristic times: the fracture mean residence time, $\frac{x_{L}}{u}$, and the characteristic time for diffusion to the center of a matrix block, $\frac{L^{2}}{4 D_{m}}$. The matrix number quantifies the relative impact of matrix diffusion on the overall transport. The importance of this parameter is discussed in Section 4.2.1.

\subsubsection{Determining Zero and First Moments}

The initial and boundary conditions for equation (4.7) are

$$
\begin{aligned}
& C_{D, N}\left(x_{D}, y_{D}, 0\right)=0 \\
& C_{D, w}\left(x_{D}, y_{D}, 0\right)=0 \\
& C_{D, w}\left(0,0, t_{D}\right)=\delta\left(t_{D}\right) \\
& C_{D, w}\left(\times, 0, t_{D}\right)=0 \\
& C_{D, m}\left(x_{D}, y_{D}, 0\right)=0 \\
& C_{D, m}\left(x_{D}, \phi_{f}, t_{D}\right)=C_{D, w} \\
& \frac{f C_{D, m}}{f y_{D}}\left(x_{D}, 1, t_{D}\right)=0
\end{aligned}
$$

Boundary condition (4.20) equates the fracture and matrix concentrations at the matrix surface while boundary condition (4.21) sets a zero concentration gradient at the center of each matrix block. 
Equation (4.9) is solved in Laplace space according to conditions (4.19) through (4.21), and differentiated to give the last term in equation (4.7):

$$
\left.\frac{f \bar{C}_{D, m}}{f y_{D}}\right|_{y_{D}=\phi_{f}}=\bar{C}_{D, w} \sqrt{\frac{s}{N_{M a}}} \tanh \sqrt{\frac{s}{N_{M a}}}\left(\phi_{f}-1\right) \sqrt[y]{ }
$$

Equation (4.22) and equation (4.8) are then substituted into equation (4.7), all in Laplace space to give:

$$
E(s) \bar{C}_{D, w}+\frac{f \bar{C}_{D, w}}{f x_{D}}-\frac{1}{N_{P e}} \frac{f^{2} \bar{C}_{D, w}}{f x_{D}^{2}}=0
$$

which is similar to equation (3.53) except for the $E(s)$ term:

$$
\begin{aligned}
& E(s)=s+\left(R_{f}-1\right)\left(\frac{s N_{D a}}{s+N_{D a}}\right) \\
& -\frac{\phi_{m}}{\phi_{f} S_{w}} \sqrt{s N_{M a}} \tanh \sqrt{\frac{s}{N_{M a}}}\left(\phi_{f}-1\right) \sqrt{ }
\end{aligned}
$$

Equation (4.23) is a linear partial differential equation, and can be solved using boundary conditions (4.17) and (4.18) to give

$$
\bar{C}_{D, w}=\exp \frac{N_{P e}-\sqrt{N_{P e}^{2}+4 N_{P e} E(s)}}{2} x_{D} \sqrt{ }
$$

Applying equation (3.56) to equation (4.25) with $x_{D}=1$, for $\mathrm{n}=0$ and $\mathrm{n}=1$ yields:

$$
\begin{aligned}
& m_{0}=1 \\
& m_{1}=R_{f}+\frac{\phi_{m}}{S_{w}}\left(\frac{1}{\phi_{f}}-1\right)
\end{aligned}
$$

The mean residence time can be calculated by dividing the zero and first moments: 


$$
\bar{t}_{D}=\frac{m_{1}}{m_{0}}=R_{f}+\frac{\phi_{m}}{S_{w}}\left(\frac{1}{\phi_{f}}-1\right)
$$

The mean residence time is expressed in fracture swept pore volumes, the $t_{D}$ defined in equation (3.32). For $R_{f}=1$, equation (4.28) reduces to the result derived by Becker (1996), which did not include water/NAPL tracer partitioning. Note that equation (4.28) indicates that the impact of matrix diffusion on the mean residence time is independent of the effective tracer diffusion coefficient in the matrix, for complete capture of the tracer response curve. The mean residence time of equation (4.28) is the sum of the mean residence time of the tracer in the fracture and the mean residence time of the tracer in the matrix. This relationship can be verified by making a simple calculation of theoretical mean residence times in the fracture and matrix for a partitioning tracer:

$$
\begin{aligned}
& \bar{t}=\frac{\text { flowable volume }}{\text { volumetric flow rate }} R_{f} \\
& \bar{t}_{f}=\frac{2 b S_{w} x_{L}}{2 b S_{w} u} R_{f}=\frac{x_{L}}{u} R_{f} \\
& \bar{t}_{m}=\frac{(L-2 b) \phi_{m} x_{L}}{2 b S_{w} u} R_{f}
\end{aligned}
$$

For the matrix, we assume no NAPL saturation, so $R_{f}=1$ and equation (4.31) can be rewritten as

$$
\bar{t}_{m}=\frac{x_{L}}{u} \frac{\phi_{m}}{S_{w}} \frac{L}{2 b}-1 \sqrt{ } \sqrt{\downarrow}=\frac{x_{L}}{u} \frac{\phi_{m}}{S_{w}} \frac{1}{\phi_{f}}-1 \sqrt{\downarrow} \sqrt{ }
$$

Equations (4.30) and (4.32) can be expressed in terms of $t_{D}$ as defined in equation (3.32) by dividing through by $\frac{x_{L}}{u}$ :

$$
t_{D f}=R_{f}
$$




$$
\begin{aligned}
& \bar{t}_{D m}=\frac{\phi_{m}}{S_{w}} \frac{1}{\phi_{f}}-1 \sqrt{ } \\
& \bar{t}_{D f}+\bar{t}_{D m}=R_{f}+\frac{\phi_{m}}{S_{w}} \frac{1}{\phi_{f}}-1 \sqrt{ }
\end{aligned}
$$

Note that the result in equation (4.35) is identical to that of equation (4.28).

Typical fracture porosities are on the order of $\phi_{f}=0.01$. For a sandstone, the matrix porosity will be on the order of 0.2 . These values yield a contribution of approximately 20 by the matrix to the overall mean residence time in equation (4.35). So for a partitioning tracer with a retardation factor in the range of $1.2<R_{f}<3.0$, the matrix diffusion has roughly an order of magnitude more effect on the tracer mean residence time than the NAPL partitioning in the fracture. However, it is important to realize that this result is based on the assumption of complete capture of the response curve over large time, an unrealistic approximation for many actual cases of interest. The following section explores the sensitivity of the model results under more realistic assumptions.

\subsection{Sensitivity Analysis}

Equation (4.28) from section 4.1.2 indicates that the impact of matrix diffusion on the first moment is independent of the effective tracer diffusion coefficient in the matrix, for complete capture of the tracer response curve. However, in practice, detection limits and time constraints prevent capture of a complete response curve. Sensitivity analyses using the model solution (equation (4.25)) can demonstrate how matrix diffusion effects coupled with mass transfer effects impact the moments calculated from a tracer response curve. Inspection of equation (4.25) yields 6 dimensionless parameter groups that may impact the model response curve: $R_{f}$, 
$N_{P e}, N_{D a}, N_{M a}, \phi_{f}$, and $\frac{\phi_{m}}{\phi_{f} S_{w}}$. The latter three parameters, which are unique to fracture transport, are analyzed in the following three subsections. The first three parameters are then added to the problem in the combined study of Section 4.3.

\subsubsection{Sensitivity Analysis $N_{M a}$}

The parameter, $N_{M a}$, quantifies the relative impact of matrix diffusion on the overall transport. Figure 4.2 shows the effect of $N_{M a}$ on the calculated mean residence time for a response curve. For this case, $R_{f}=1$ to eliminate effects of water/NAPL partitioning. When $N_{M a}=1$, the tracer is in equilibrium with the surrounding matrix, so the theoretical mean residence time given in equation (4.35) results ( $\bar{t}_{D}$ in Fig. 4.2). When $N_{M a}=0.001$, there is little interaction between the tracer and the surrounding matrix, so the fracture mean residence time given by equation (4.33) results ( $\bar{t}_{D f}$ in Fig. 4.2). For $N_{M a}=0.05$, the MRT at $t_{D}=15$ falls between the total and fracture MRTs. Note that for this illustrative case, the matrix only doubles the total volume of the system, since fracture spacing is small. For most real cases, the fracture spacing (L) would be large compared to the fracture aperture, and the fracture porosity $\frac{2 b}{L} V_{\downarrow}$ correspondingly smaller, which would result in a larger difference between the fracture and total MRTs. Figure 4.3 shows the tracer response curves that correspond to the MRT curves of Fig. 4.2. Note the nonequilibrium evidenced in the curve for $N_{M a}=0.05$ (early breakthrough with late time tailing). Table 4.1 gives a summary of the input values for the model curves. 


\section{$\underline{4.2 .2 \text { Sensitivity Analysis } \phi_{f}}$}

The fracture porosity appears in two places in equation (4.25) --individually in the hyperbolic tangent term, and grouped with the matrix porosity and water saturation. The term in the hyperbolic tangent is $\left(\phi_{f}-1\right)$, and the fracture porosity is typically small, i.e., $0.01-0.1$. Therefore, the relative change of this term over the typical range of fracture porosities will be small.

The individual impact of this fracture porosity term on the tracer output curves was determined by varying the fracture porosity while keeping the parameter group $\frac{\phi_{m}}{\phi_{f} S_{w}}$ constant. Figures 4.4 and 4.5 show the tracer concentration response curves at three fracture porosities, with $N_{M a}=1.0$ and $N_{M a}=0.001$. As expected, varying the fracture porosity without varying $\frac{\phi_{m}}{\phi_{f} S_{w}}$ had very little effect on the tracer response at both low and high matrix numbers. Table 4.2 gives a summary of the input parameters for all of the model curves.

$\underline{4.2 .3 \text { Sensitivity Analysis }} \frac{\phi_{m}}{\phi_{f} S_{w}}$

This parameter group would be expected to affect two characteristics of tracer transport in the fracture. First, the relative volumes of the fracture and matrix are given by the parameters in this group. The fracture porosity specifies the fraction of the bulk volume of the media that is fracture volume. The matrix porosity describes the ratio of the pore matrix volume to the total matrix volume. The small relative variation in the water saturation has a correspondingly small effect on the volume of the fracture that is mobile fluid. The relative variation in water saturation is small because NAPL saturations are typically less than $5 \%$. 
High matrix number. Consider the case where the matrix number is high (i.e., $N_{M a}=$ 1.0), so the tracer flowing in the fracture is close to equilibrium with the matrix. A decrease in the fracture porosity gives a relative increase in the volume of the matrix compared to the volume of the fracture, so the mean residence time (in terms of fracture volumes) would increase. This trend is supported in equation (4.35) where the fracture porosity is in the denominator. An increase in the matrix porosity would also increase the pore volume of the matrix compared to the fracture volume, so again the mean residence time would increase. This trend is shown in equation (4.35), where the matrix porosity is in the numerator. An increase in the water saturation decreases the fracture volume, which increases the mean residence time because the mean residence time is expressed in terms of fracture swept volumes. In summary, at high matrix number, an increase in the parameter group $\frac{\phi_{m}}{\phi_{f} S_{w}}$ will result in a corresponding increase in the mean residence time of the tracer.

Figure 4.6 shows the increase in the cumulative tracer MRT with increasing $\frac{\phi_{m}}{\phi_{f} S_{w}}$. These curves verify the trends predicted in the preceding analysis. Figure 4.7 shows the corresponding tracer response curves. Note the linear decline on the semi-log plot, which indicates equilibrium for the tracer between the fracture and matrix. The first three rows of Table 4.3 summarize the input parameters corresponding to the model curves.

Low matrix number. At lower matrix number, local equilibrium does not occur between the fracture and matrix. If the matrix number is very low, with no tracer interaction with the matrix, then the mean residence time will always be equal to 1 fracture pore volume (Section 4.2.1), regardless of the parameter values. However, if the matrix number is such that the tracer has some interaction with the matrix, but is not at equilibrium with the matrix, then increasing 
$\frac{\phi_{m}}{\phi_{f} S_{w}}$ will increase the impact of matrix diffusion on the output curve. The increase in the impact of matrix diffusion is due to the increasing fracture wall surface area per unit volume of fracture. The higher the fracture wall surface area for a given volume of fracture, the more impact matrix diffusion has on tracer transport.

For example, given an increase in the fracture aperture (and correspondingly the fracture porosity), the fracture volume per unit length increases while the fracture wall surface area per length remains constant. With the constant surface area, the rate of mass diffusion into the matrix remains constant. However, with the increased fracture volume, for a given tracer concentration, more tracer mass is available in the fracture. Therefore, a smaller relative decrease in tracer concentration in the fracture occurs with a correspondingly smaller relative impact on the tracer response curve. An increase in the matrix porosity increases the fraction of fracture wall surface area that is available for solute transfer from the fracture.

Figure 4.8 shows the increasing effect of matrix diffusion with increasing $\frac{\phi_{m}}{\phi_{f} S_{w}}$, where $N_{M a}=0.001$. Figure 4.9 further illustrates the trend as seen in the tracer response curves. With increasing $\frac{\phi_{m}}{\phi_{f} S_{w}}$, the response curves tail more, because matrix diffusion has a higher overall effect on the tracer transport in the fracture. The last three rows of Table 4.3 summarize the input parameters corresponding to the model curves.

\subsection{Combined Impacts of Matrix Diffusion and WaterINAPL Mass Transfer}

The sensitivity analyses of sections 4.2.1-4.2.3 give information about fracture transport with matrix diffusion. However, an evaluation of the effectiveness of PITTs in fractured media 
required a combined analysis of both water/NAPL tracer mass transfer and matrix diffusion. The most important variables in the analyses were the Damkohler number, the matrix number, and the fracture porosity.

\subsubsection{Error in First Moment of the Partitioning Tracer}

Multiple sensitivity runs were made, varying the Damkohler and matrix numbers for a given fracture porosity. Figures 4.10-4.18 show contour plots of the percent error in the fracture mean residence time predicted by the partitioning tracer response curve with varying Damkohler number and matrix number. The shading reflects chart areas with different percent error ranges - errors of less than 5\% (white), between 5\% and 10\% (light gray), and $10 \%$ or greater (dark gray). These errors are based on the assumption that the fracture mean residence time is the desired variable to be calculated from the tracer response curves.

Figure 4.8 shows that a Damkohler number of approximately 1 or higher and a matrix number of approximately $5 \times 10^{-6}$ or lower are required for less than $5 \%$ error in the calculated fracture mean residence time, for a fracture porosity of 0.01 . Increasing the fracture porosity to 0.05 increases the $5 \%$ cutoff more than an order of magnitude on the matrix number axis (Fig. 4.11). The same trend is shown in Fig. 4.12 when the fracture porosity is increased to 0.1. In general, an increase in the fracture porosity results in better estimates of the fracture mean residence time at a given matrix number.

\subsubsection{Error in Calculated Retardation Factor}

The retardation factor for a partitioning tracer is calculated by dividing the mean residence time of that tracer by the mean residence time of the conservative tracer. The retardation factor can be used to calculate the NAPL saturation in the fracture (i.e., equation 2.6). The error in the NAPL 
saturation estimate will be proportional to the error in the calculated retardation factor. In a typical partitioning tracer test, the conservative tracer is a smaller molecule than the partitioning tracers, and therefore has a higher diffusion coefficient.

Figures 4.13 and 4.14 show the relative error in the calculated retardation factor, for two cases. In the first case, the diffusion coefficient of the partitioning tracer is the same as that of the conservative tracer. In the second case, the diffusion coefficient of the conservative tracer is twice that of the partitioning tracer. Figure 4.12 shows that for the second case, the $5 \%$ cutoff was transposed nearly an order of magnitude lower on the matrix number axis. Figures 4.15 4.18 show similar trends for other values of fracture porosity. The increase in fracture porosity improved the estimate of the retardation factor equally for both cases. In general, when the conservative tracer has a higher diffusion coefficient than the partitioning tracer, the error in the calculated retardation factor will increase.

\subsection{Summary and Conclusions}

A 1-D parallel fracture model which includes partitioning tracer water/NAPL mass transfer was derived. Dimensional analysis of the equations for this model resulted in a new parameter, termed the matrix number, that describes the ratio of theoretical fracture mean residence time to the time required to diffuse to the center of the matrix.

The zero and first moments were calculated for the model solution in Laplace space. The results indicated that the theoretical tracer mean residence time is independent of the matrix and Damkohler numbers. The fracture and matrix contributions to the mean residence time were separated and discussed. If the tracer response curve is complete, the matrix contribution to the mean residence time dominates the overall mean residence time, for typical fractured media. 
Sensitivity analyses with the model indicate variable accuracy in NAPL saturation estimates made from partitioning tracer data in fractured media, depending on the fracture porosity, matrix porosity, and matrix number. In general, high fracture porosities, low matrix porosities, and low matrix numbers all improve the saturation estimate, because the overall mean residence time approaches the theoretical fracture mean residence time.

Sensitivity analyses also indicated that using conservative tracers with diffusion coefficients greater than the partitioning tracers could significantly increase the error in the saturation estimates.

\subsection{Tables}

Table 4.1: Parameter Values for Model Runs Described in Section 4.2.1

\begin{tabular}{|c|c|c|c|c|c|}
\hline$N_{M a}$ & $\frac{\phi_{m}}{\phi_{f} S_{w}}$ & $\phi_{f}$ & $N_{D a}$ & $N_{P e}$ & $R_{f}$ \\
\hline \hline 1.0 & 1.1 & 0.1 & 100 & 5 & 1.0 \\
\hline 0.05 & 1.1 & 0.1 & 100 & 5 & 1.0 \\
\hline 0.001 & 1.1 & 0.1 & 100 & 5 & 1.0 \\
\hline
\end{tabular}

Table 4.2: Parameter Values for Model Runs Described in Section 4.2.2

\begin{tabular}{|c|c|c|c|c|c|}
\hline$\phi_{f}$ & $\frac{\phi_{m}}{\phi_{f} S_{w}}$ & $N_{M a}$ & $N_{D a}$ & $N_{P e}$ & $R_{f}$ \\
\hline \hline 0.1 & 2.2 & 1.0 & 100 & 5 & 1.0 \\
\hline 0.05 & 2.2 & 1.0 & 100 & 5 & 1.0 \\
\hline 0.01 & 2.2 & 1.0 & 100 & 5 & 1.0 \\
\hline 0.1 & 2.2 & 0.001 & 100 & 5 & 1.0 \\
\hline 0.05 & 2.2 & 0.001 & 100 & 5 & 1.0 \\
\hline 0.01 & 2.2 & 0.001 & 100 & 5 & 1.0 \\
\hline
\end{tabular}


Table 4.3: Parameter Values for Model Runs Described in Section 4.2.3

\begin{tabular}{|c|c|c|c|c|c|}
\hline$\frac{\phi_{m}}{\phi_{f} S_{w}}$ & $N_{M a}$ & $\phi_{f}$ & $N_{D a}$ & $N_{P e}$ & $R_{f}$ \\
\hline \hline 1.1 & 1.0 & 0.1 & 100 & 5 & 1.0 \\
\hline 2.2 & 1.0 & 0.05 & 100 & 5 & 1.0 \\
\hline 11.1 & 1.0 & 0.01 & 100 & 5 & 1.0 \\
\hline 1.1 & 0.001 & 0.1 & 100 & 5 & 1.0 \\
\hline 2.2 & 0.001 & 0.05 & 100 & 5 & 1.0 \\
\hline 11.1 & 0.001 & 0.01 & 100 & 5 & 1.0 \\
\hline
\end{tabular}

\subsection{Figures}

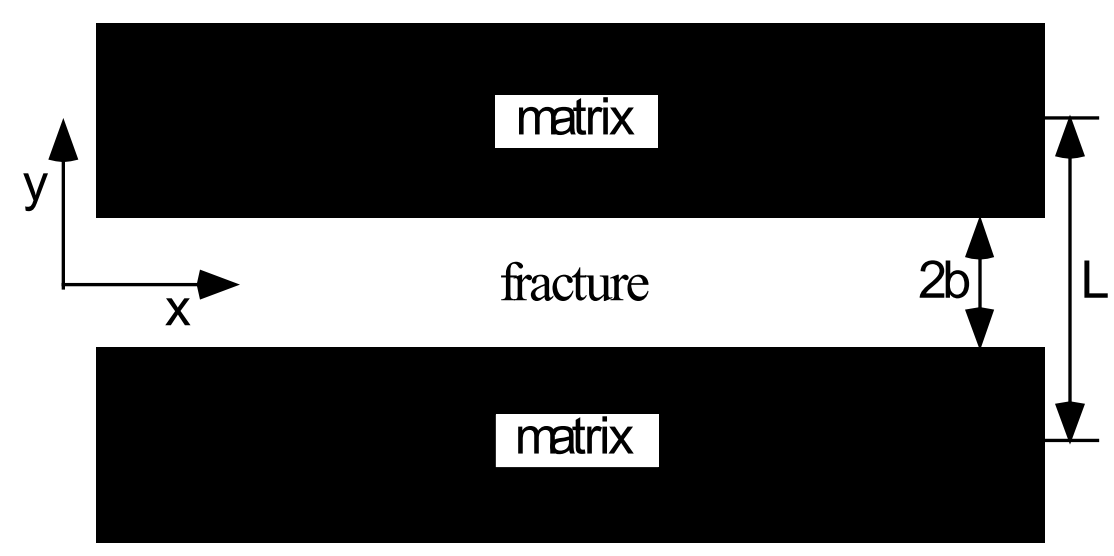

fracture

\section{matrix}

Figure 4.1: Parallel fracture model. 


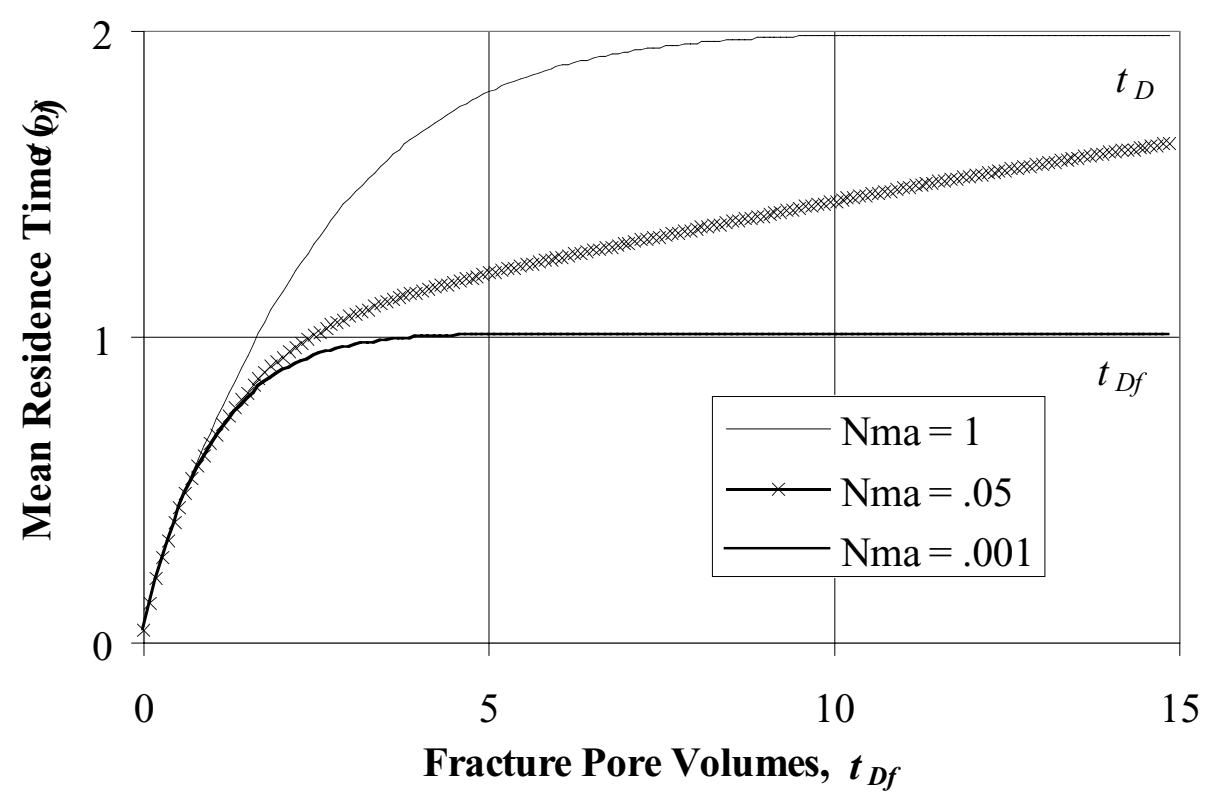

Figure 4.2: Effect of $N_{M a}$ on calculated Mean Residence Time (MRT).

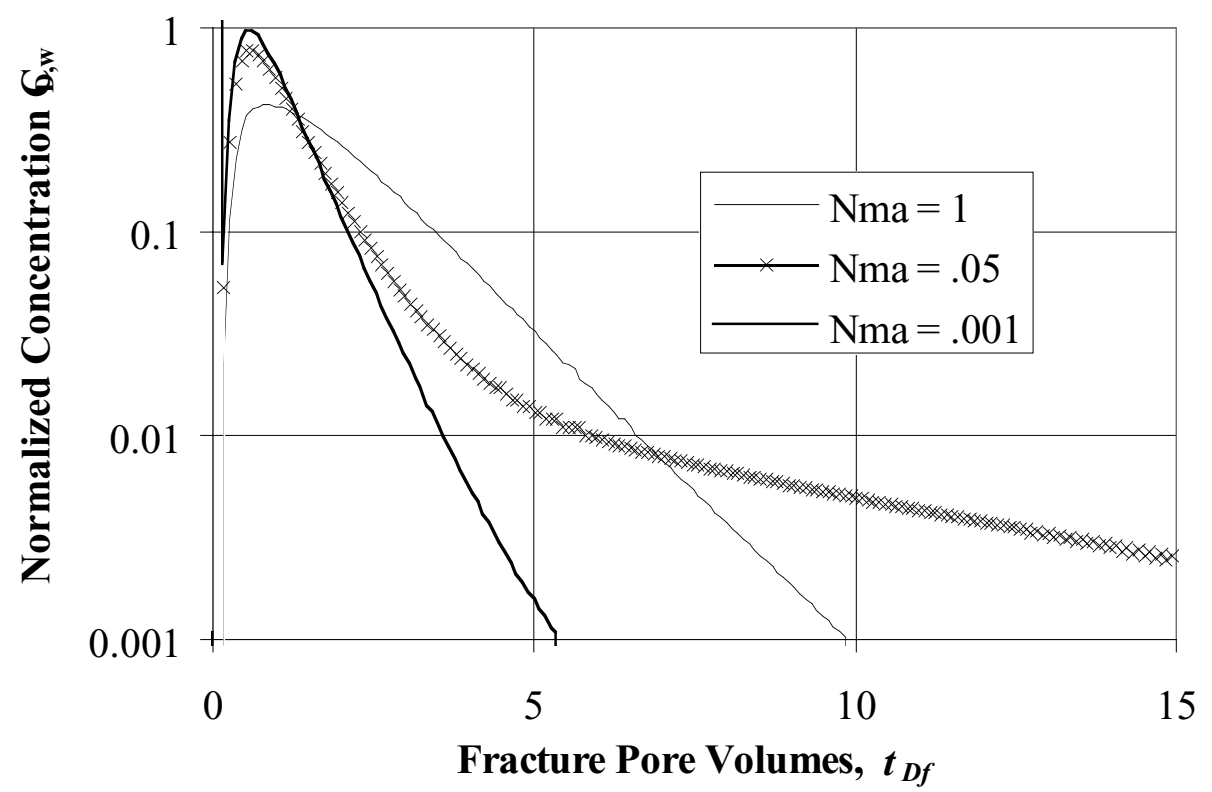

Figure 4.3: Tracer response curves at varying $N_{M a}$ values. 


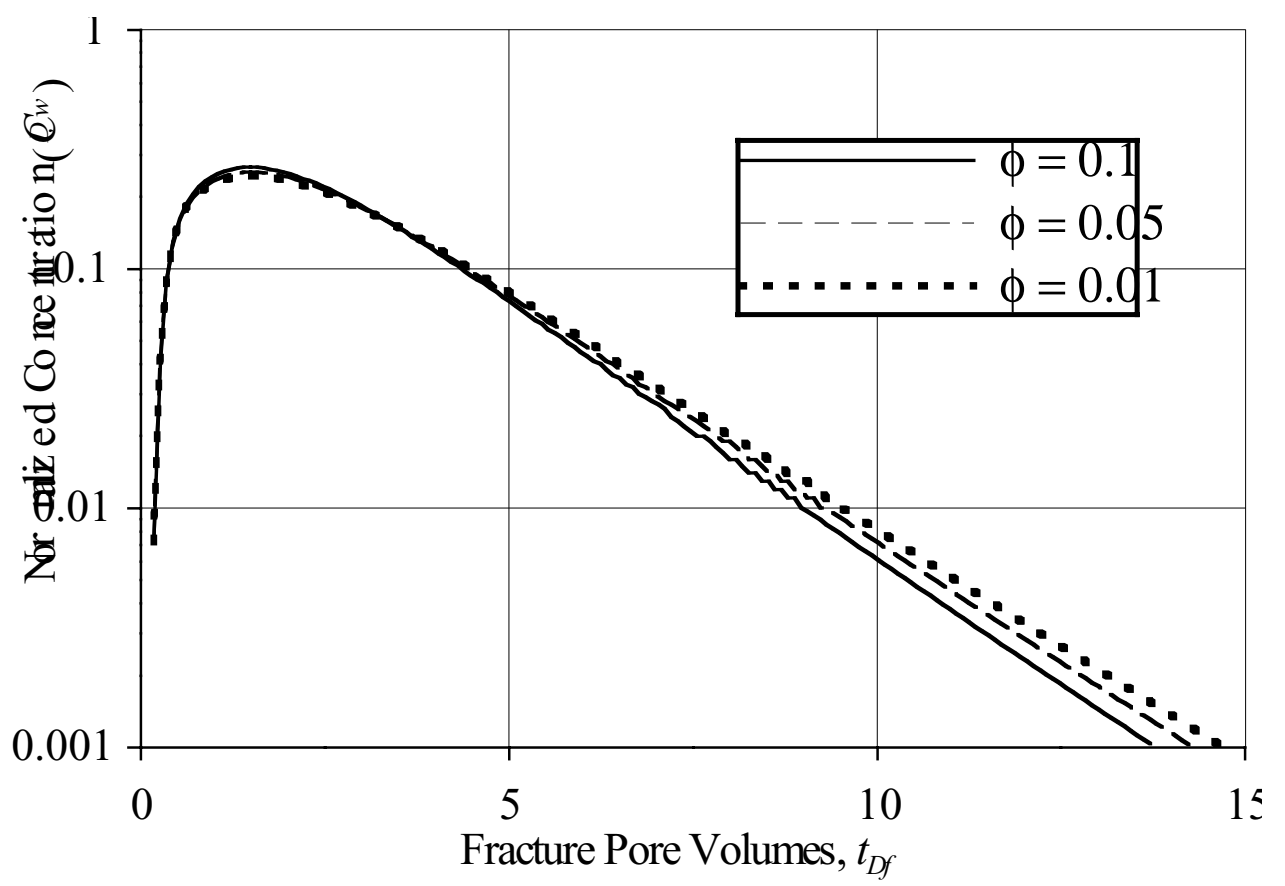

Figure 4.4: Tracer response curves at varying fracture porosity, $N_{M a}=1$.

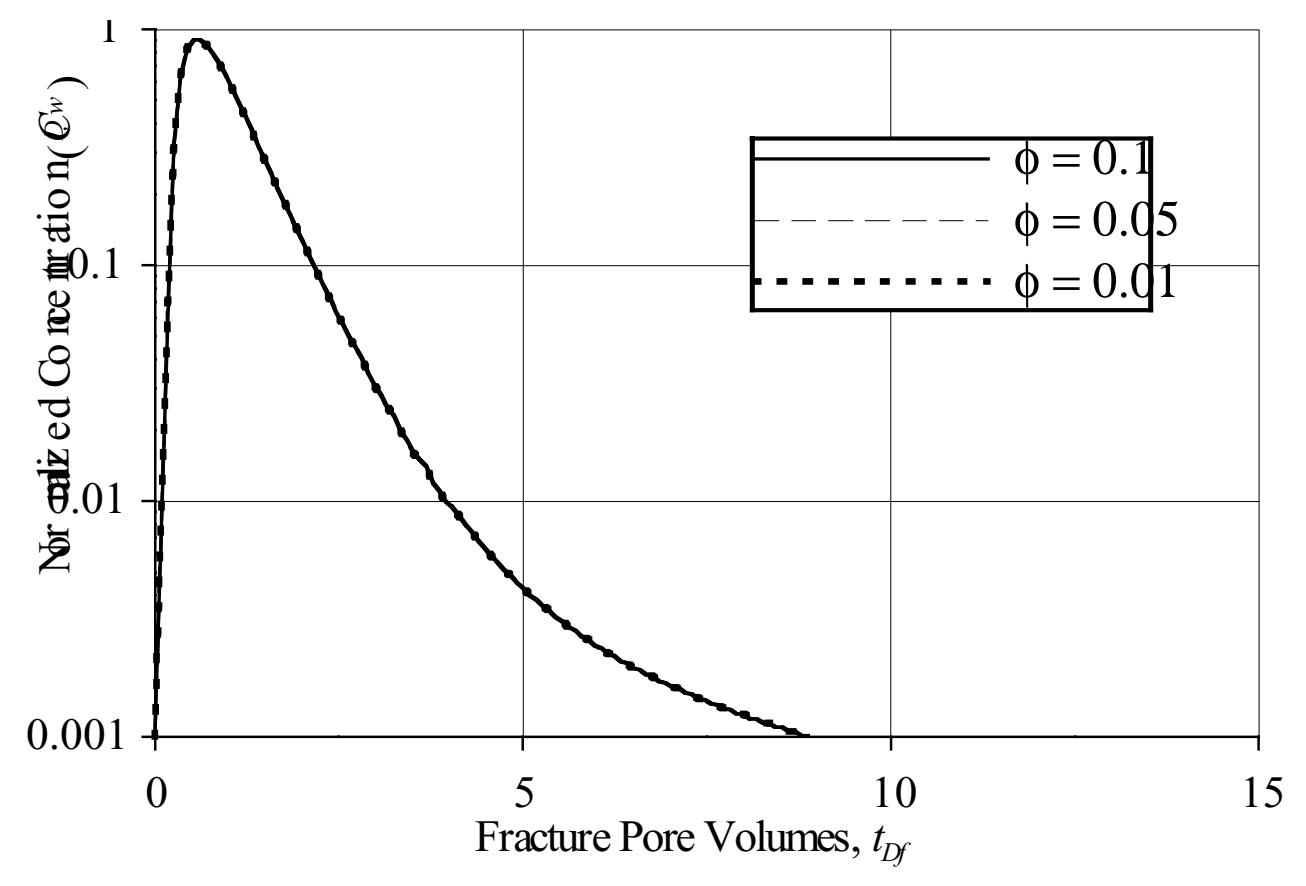

Figure 4.5: Tracer response curves at varying fracture porosity, $N_{M a}=0.001$. 


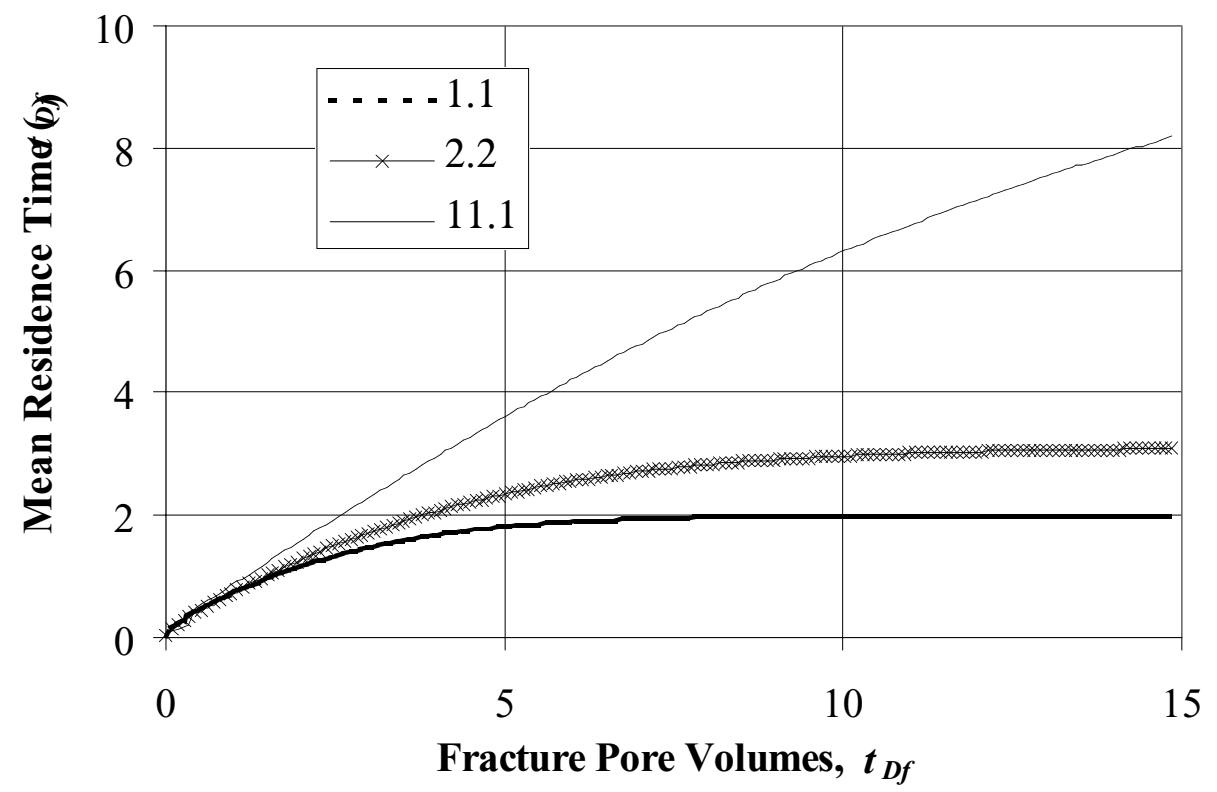

Figure 4.6: Effect of varying $\phi_{m} /\left(\phi_{f} S_{w}\right)$ on MRT at $N_{M a}=1$.

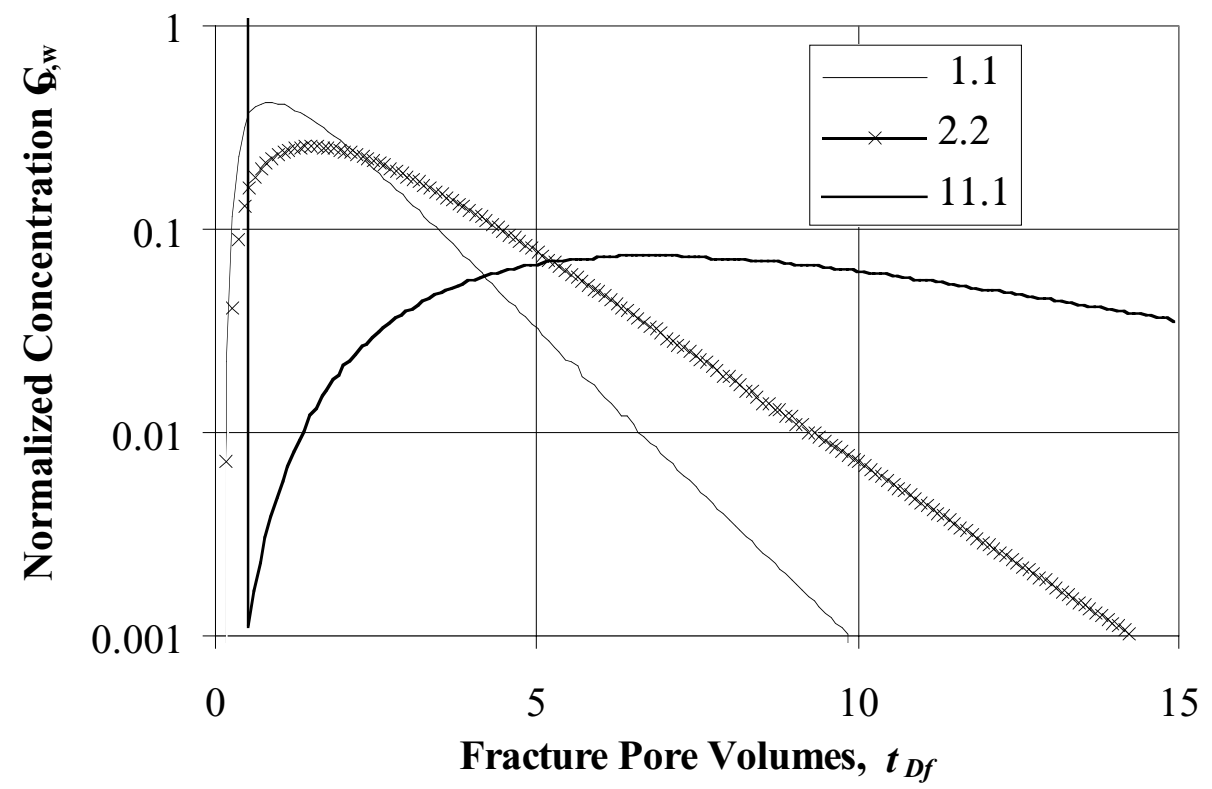

Figure 4.7: Tracer response curves at varying $\phi_{m} /\left(\phi_{f} S_{w}\right)$ values, $N_{M a}=1$. 


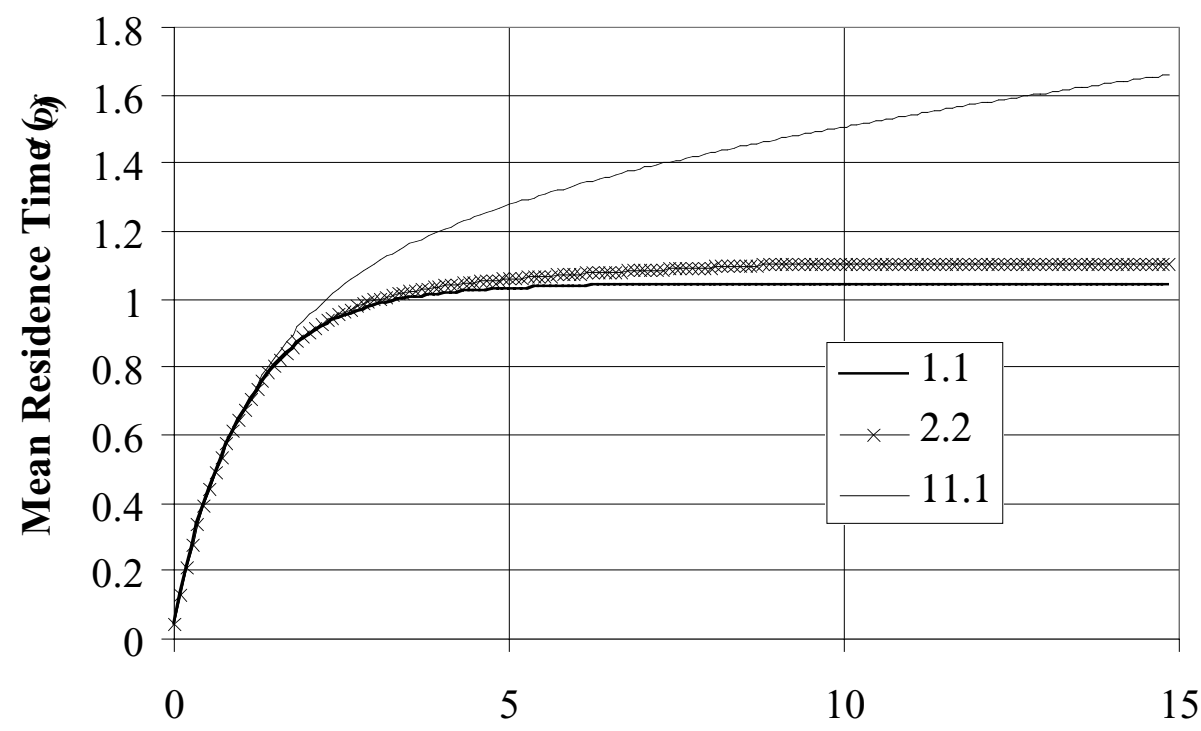

Fracture Pore Volumes, $t_{D f}$

Figure 4.8: Effect of varying $\phi_{m} /\left(\phi_{f} S_{w}\right)$ on MRT at $N_{M a}=1$.

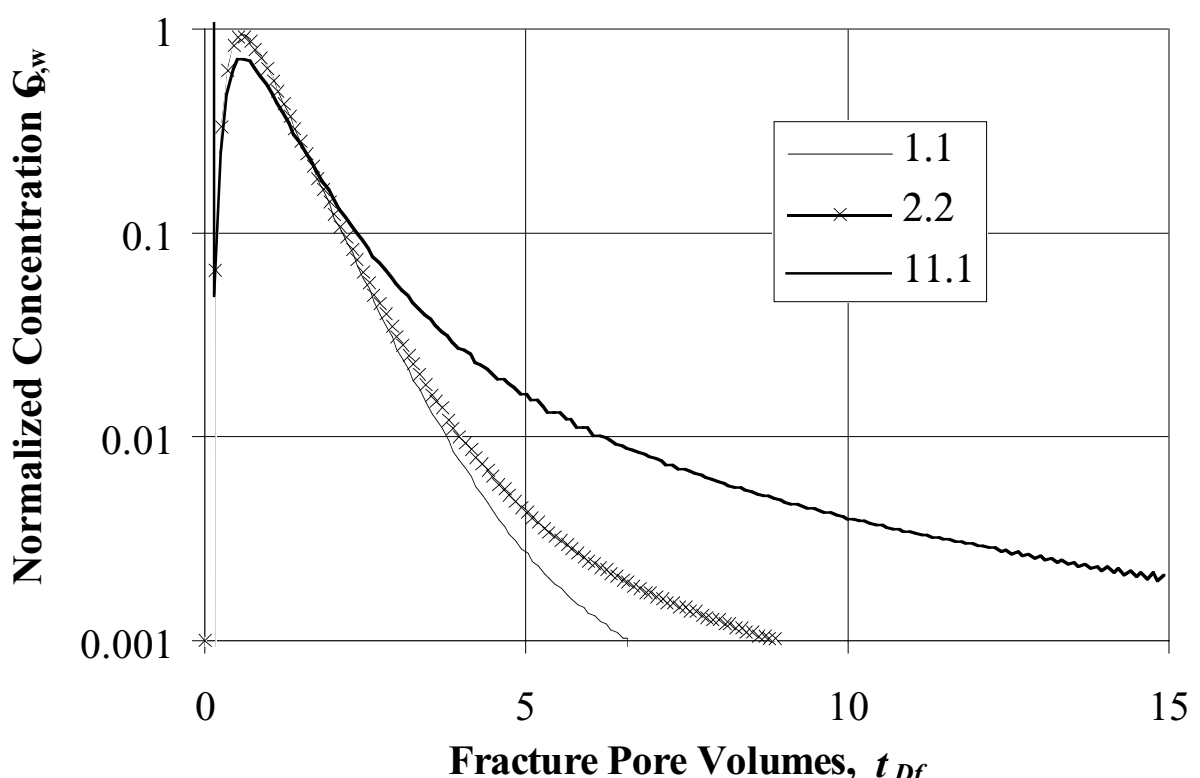

Figure 4.9: Tracer response curves at varying $\phi_{m} /\left(\phi_{f} S_{w}\right)$ values, $N_{M a}=1$. 


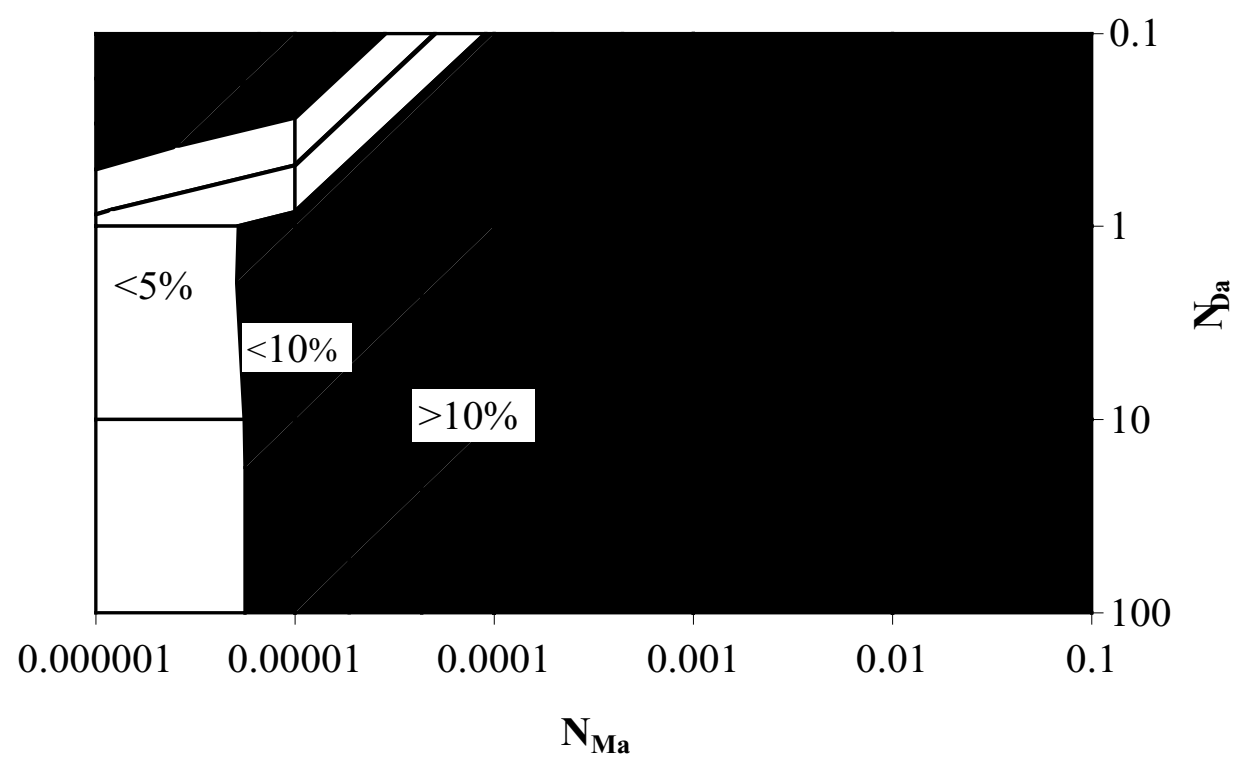

Figure 4.10: Error in calculated first moment, where $\phi_{f}=0.01$.

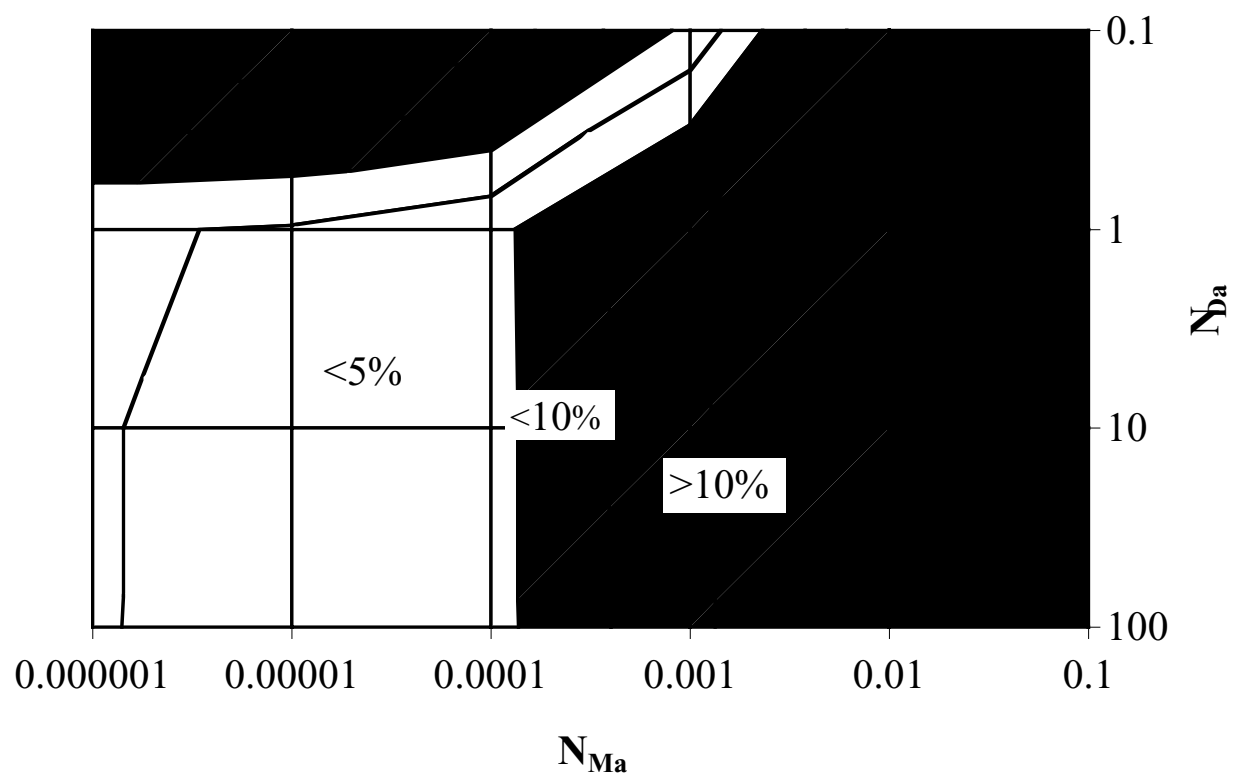

Figure 4.11: Error in calculated first moment, where $\phi_{f}=0.05$. 


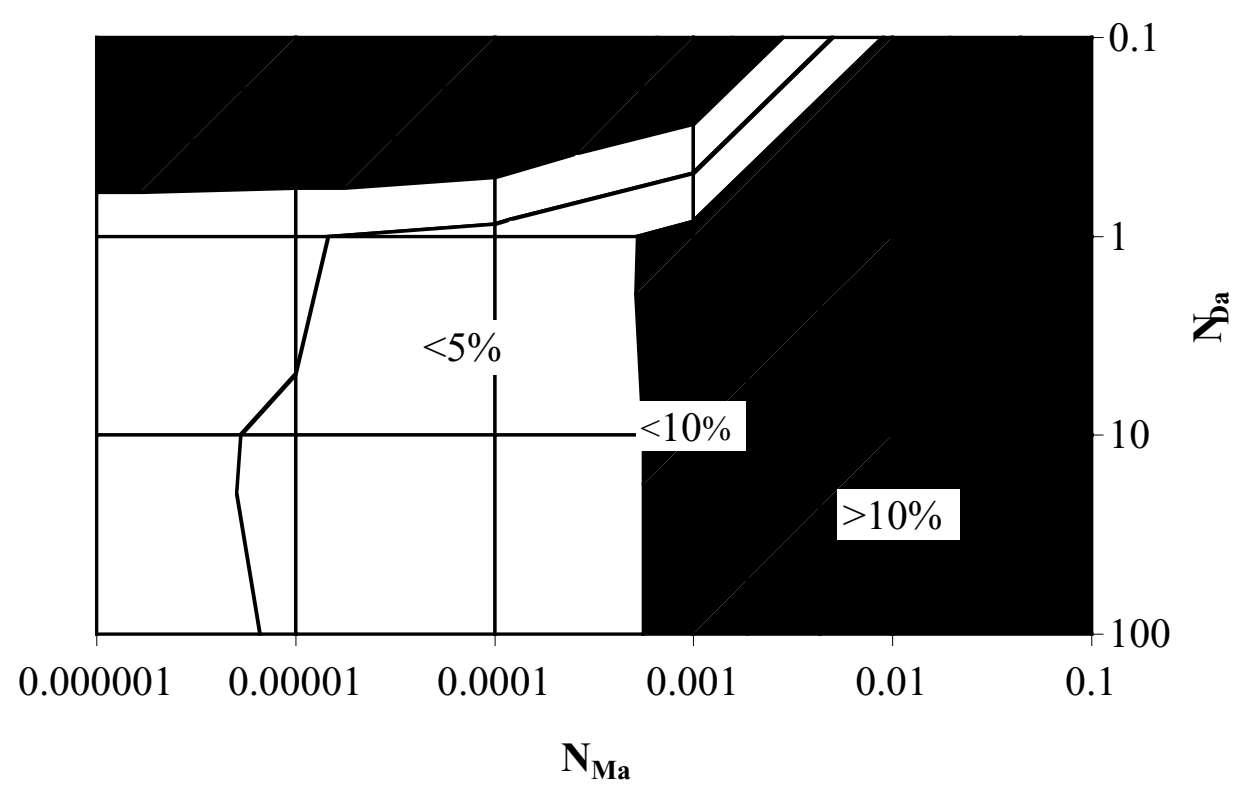

Figure 4.12: Error in calculated first moment, where $\phi_{f}=0.1$.

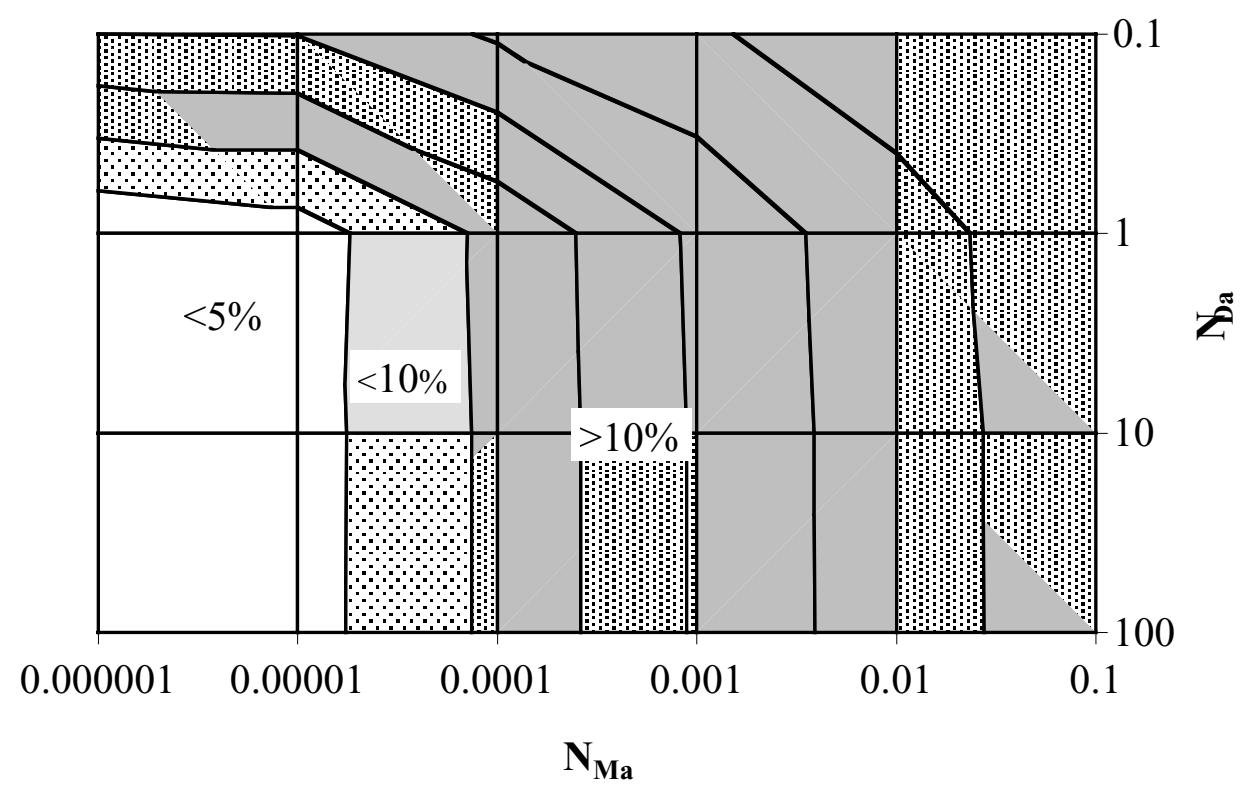

Figure 4.13: Error in retardation factor, where $D_{m 1}=D_{m 2}$ and $\phi_{f}=0.01$. 


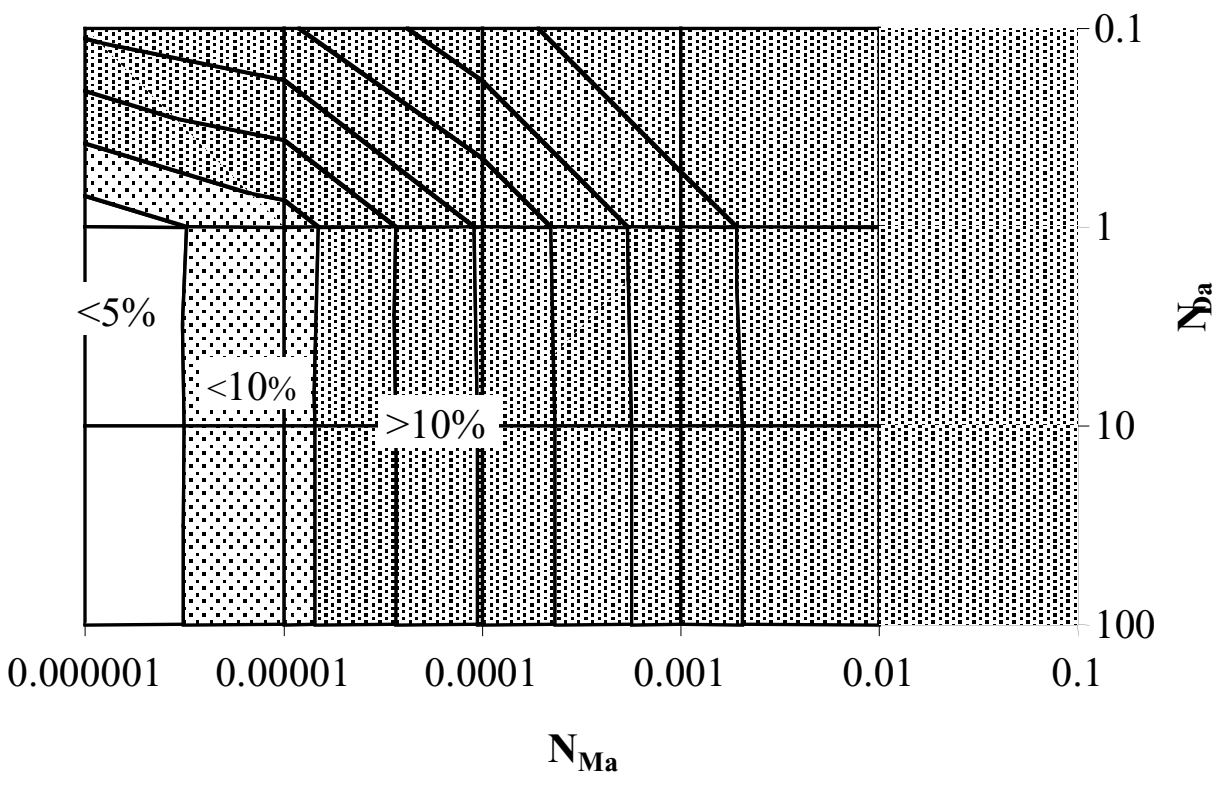

Figure 4.14: Error in retardation factor, where $D_{m 1}=2 D_{m 2}, \phi_{f}=0.01$.

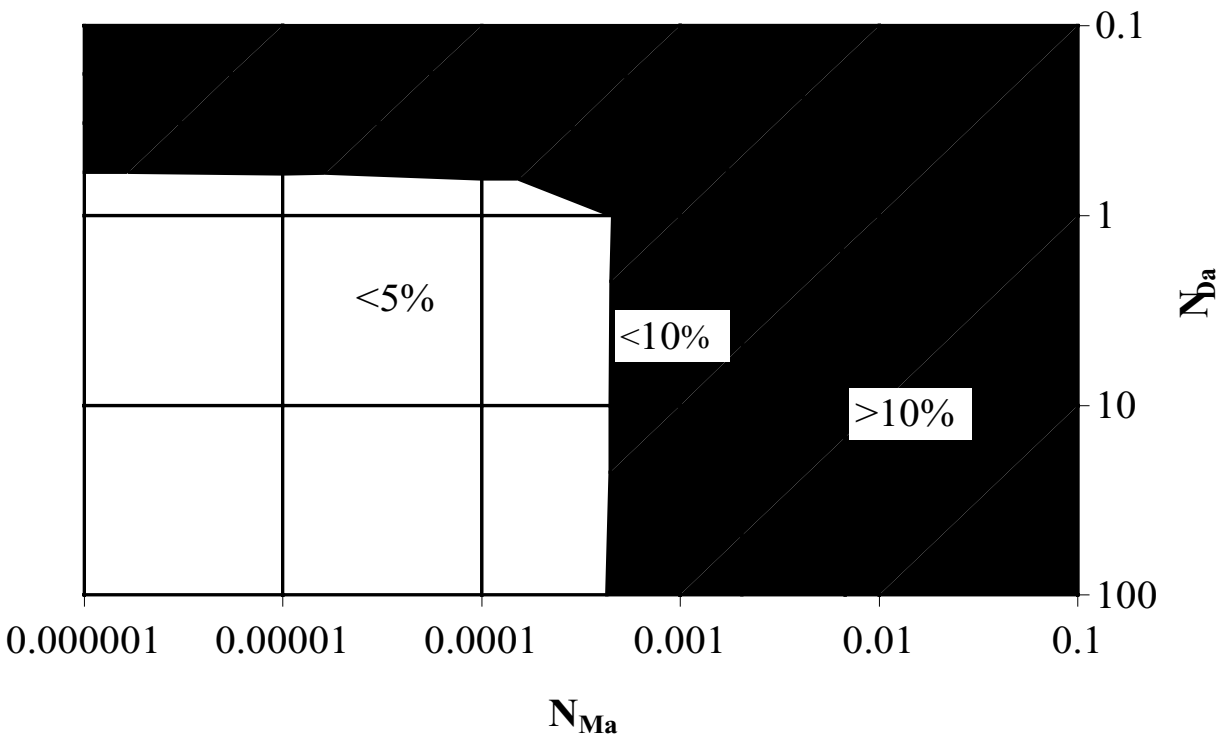

Figure 4.15: Error in retardation factor, where $D_{m 1}=D_{m 2}, \phi_{f}=0.05$. 


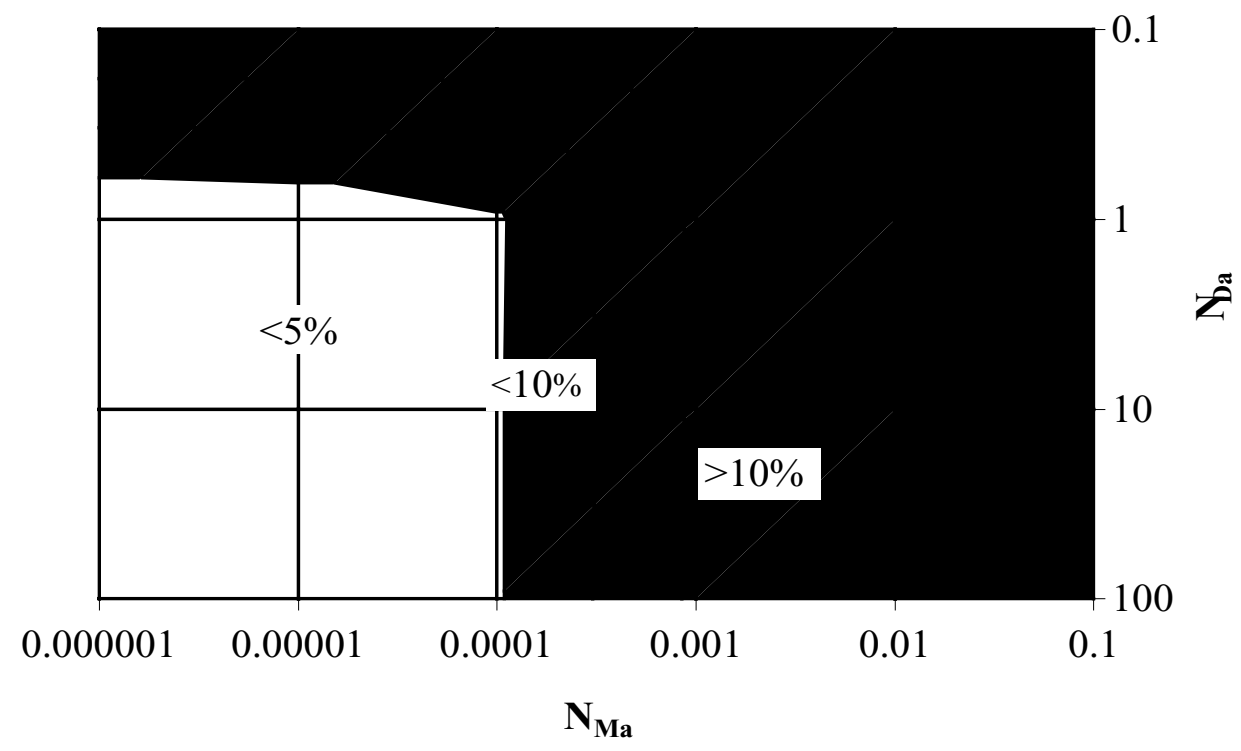

Figure 4.16: Error in retardation factor where $D_{m 1}=2 D_{m 2}, \phi_{f}=0.05$.

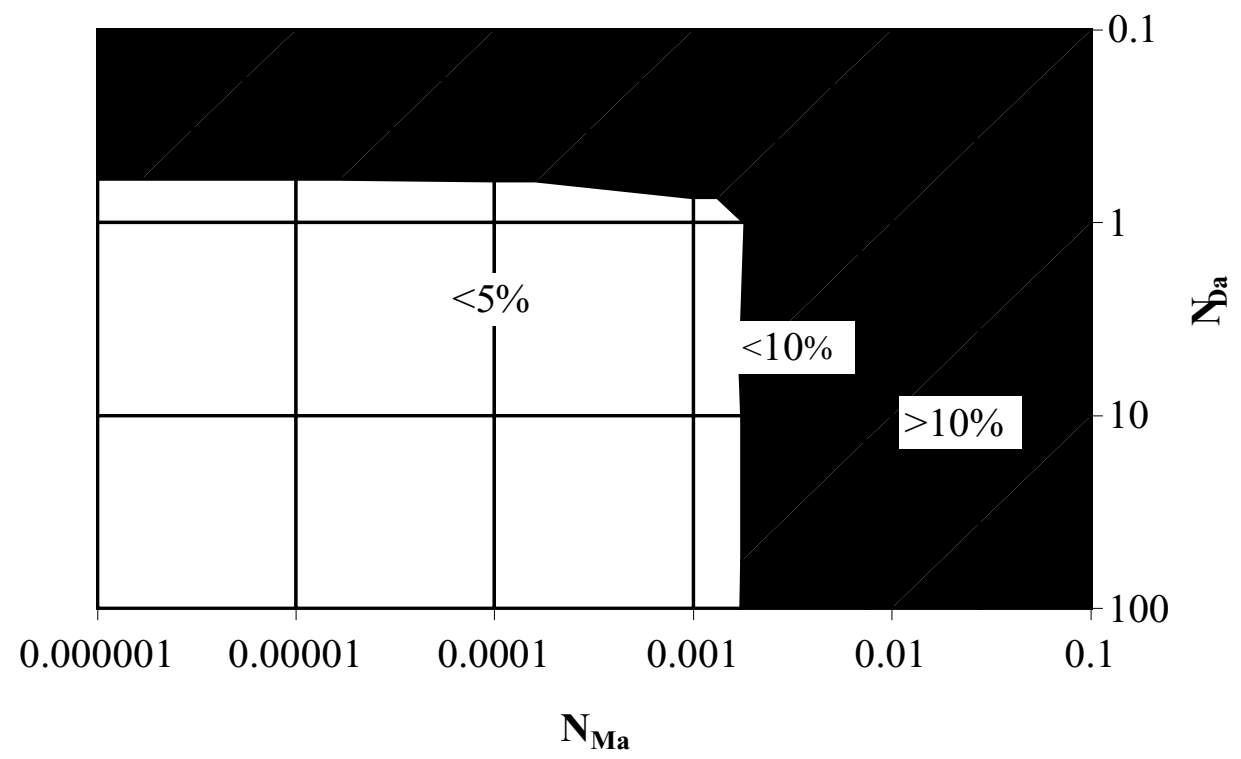

Figure 4.17: Error in retardation factor, where $D_{m 1}=D_{m 2}$ and $\phi_{f}=0.1$. 


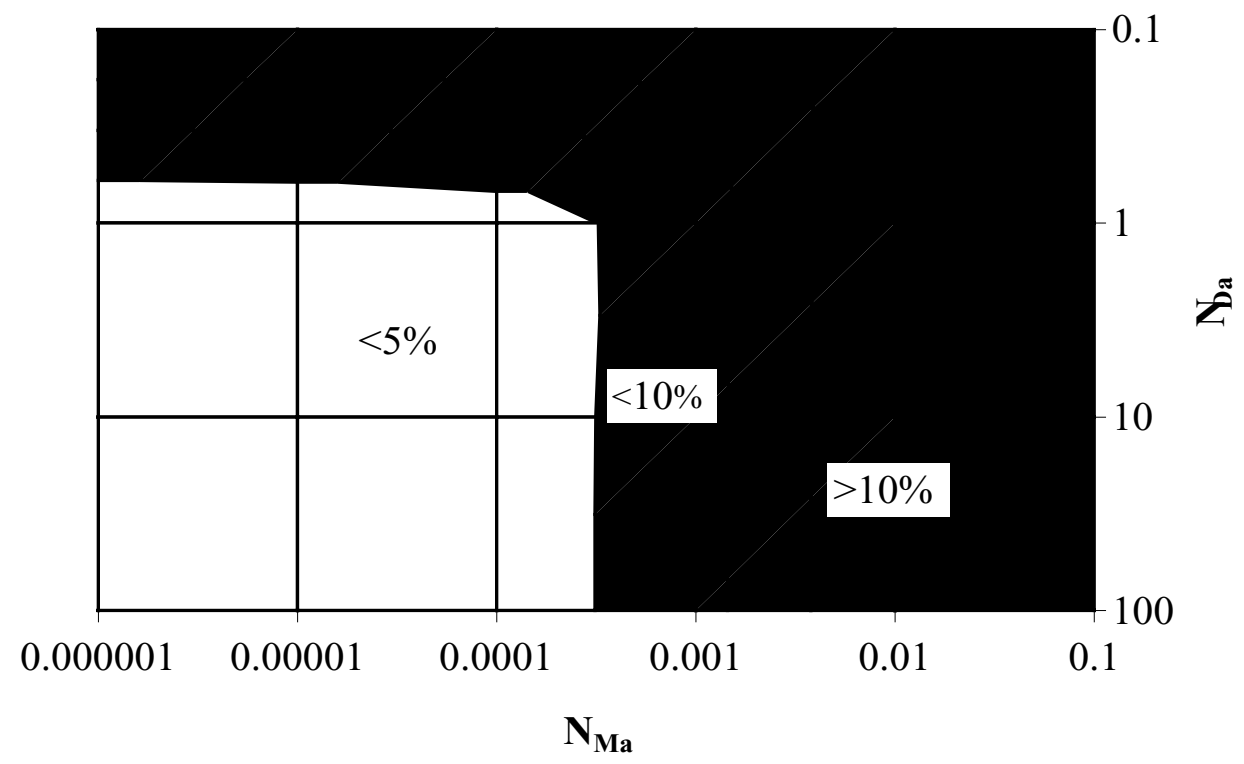

Figure 4.18: Error in retardation factor, where $D_{m 1}=2 D_{m 2}$ and $\phi_{f}=0.1$. 


\section{Section 5: Single Fracture Core Experiments}

\subsection{Introduction}

This section details several laboratory partitioning tracer experiments done in a porous rock with a single fracture. The goal of these experiments was to investigate the effects of matrix diffusion on partitioning tracer results in fractured media. As of this writing, these experiments are the first partitioning tracer tests completed in fractured media. A semi-analytical transport model similar to the model in Section 4 is used to help interpret the results.

\subsection{Experimental Materials}

\section{$\underline{5.2 .1 \text { Tracers }}$}

Six alcohols were used as tracers in these experiments, including methanol, 1-propanol, isopropanol, 1-hexanol, 1-heptanol, and 1-octanol. The latter four tracers were previously established as suitable tracers for NAPL/water partitioning tracer tests (Dwarakanath, 1997; Edgar, 1997), with isopropanol as the non-partitioning tracer and 1-hexanol, 1-heptanol, and 1octanol as partitioning tracers. Methanol and 1-propanol, as well as two radioactive tracers, tritiated water and chloride-36 were also used in the matrix diffusion studies. The alcohol tracer compounds were acquired from Aldrich Chemical, Milwaukee, WI. The radioisotopes were acquired from American Radiolabeled Chemicals, St. Louis, MO.

Table 5.1 shows some of the physical properties of these tracers. The solubility of the compounds in water is of practical importance for obtaining adequate injected concentrations. The bulk diffusion coefficient in water is important to the matrix diffusion effects. The bulk diffusion coefficients in Table 5.1 are measured values taken from Yaws (1995). 


\subsubsection{LNAPL}

Decane was used as the experimental NAPL. Although trichloroethylene (TCE) has been tested extensively with the alcohol partitioning tracers, TCE was found to degrade the epoxy that was used to seal the cores. With a density of $0.73 \mathrm{~g} / \mathrm{cm}^{3}$, decane is considered to be a light NAPL or LNAPL. Decane has a very low solubility in water. This low solubility in combination with a low water flow rate ensured that there would not be significant losses due to dissolution during the course of the experiments. The decane used in these experiments was obtained from Aldrich Chemical, Milwaukee WI.

\subsubsection{Fractured Rock}

Basically, two rock properties were important in these single-fracture experiments. First, the permeability of the rock matrix, relative to the fracture permeability, determines if there is significant advective flow in the rock matrix. Second, the tortuosity of the rock matrix affects the rate of solute diffusion to and from the fracture. Tortuosity in this case is defined as $D_{m} / D_{w}$, where $D_{m}$ is the diffusion coefficient in the matrix, and $D_{w}$ is the bulk diffusion coefficient in water (i.e., Bear, 1972). Tortuosities range from 0.001 for some types of granite to values near 0.5 for some sandstones.

Berea sandstone (Cleveland Quarries, Amhert $\mathrm{OH}$ ) was chosen for this study because it has low matrix permeability (approximately $0.1 \mathrm{D}$ or $10^{-4} \mathrm{~cm} / \mathrm{s}$ ) relative to the expected fracture permeability (>100 D). Berea also has a high tortuosity of 0.4 (Donaldson et al., 1976), which ensured the possibility of significant matrix diffusion effects. 


\subsection{Experimental Methods}

\subsubsection{Determination of Partition Coefficients}

Equation (2.3) gives the definition of a partition coefficient. Batch equilibrium experiments were used to measure the partition coefficients between each partitioning tracer and decane. In general, the static partition coefficient test involves adding an aqueous solution of tracer to a known volume of NAPL, allowing equilibration, and testing for the final aqueous tracer concentration (Dwarakanath, 1997; Dwarakanath and Pope, 1998). The specific procedure was as follows:

1. First, a rough estimate of the partition coefficient was made to help design the experiment. The estimate needed only to be accurate within half an order of magnitude of the true value. Simple estimates were made by reviewing similar tracer/NAPL pairs. A recent correlation developed by Dwarakanath and Pope (1998) could be used for a large number of alcohols and NAPLs.

2. Values for the experimental parameters were determined by targeting a final aqueous tracer concentration that was well above the detection limit for the tracer. For the alcohols used in the fracture experiments, the final aqueous concentrations should fall in the approximate range of 25 to $250 \mathrm{mg} / \mathrm{L}$. Isotherms are linear in this dilute range, i.e., the partition coefficients are constant.

3. The final aqueous concentration was estimated by the following equation:

$$
C_{F, w}=\frac{C_{I, w}}{\frac{V_{N} K_{N}}{V_{w}}+1}
$$

where 


$$
\begin{aligned}
C_{F, w} & =\text { final tracer concentration in water } \\
C_{I, w} & =\text { initial tracer concentration in water } \\
V_{N} & =\text { volume of NAPL } \\
V_{w} & =\text { volume of water } \\
K_{N} & =\text { estimated partition coefficient between NAPL and water }
\end{aligned}
$$

Several vials of increasing aqueous concentration were made to ensure that several of the final aqueous concentrations fell inside the target range, allowing for the variability of the original partition coefficient estimate. The multiple vials also provided redundancy to check the precision of the measurements.

4. The aqueous tracer solution and NAPL were combined in a screw cap vial and agitated by wrist action shaker until the NAPL broke into fine droplets. The dispersion of the NAPL in the droplets created a large amount of surface area for equilibrium exchange of the tracer between the two phases.

5. The vials were allowed to equilibrate for at least 24 hours. They were then centrifuged to ensure complete separation of the phases. The water concentration was sampled and analyzed by gas chromatography (GC) using a model 610 portable GC (Buck Scientific, East Norwalk, CT) equipped with a flame ionization detector. The GC column was a 30 m AT-Wax (Alltech, Deerfield, IL) at $105^{\circ} \mathrm{C}$ with a nitrogen carrier flow of $3 \mathrm{~mL} / \mathrm{min}$. The partition coefficient value was determined by rearrangement of equation (5.1):

$$
K_{N}=\frac{V_{N}}{V_{W}} \frac{C_{I, W}}{C_{F, W}}-1 \sqrt{ }
$$




\subsubsection{Preparation of Fractured Core}

1. Berea sandstone was available in $5 \mathrm{~cm}$ width by $5 \mathrm{~cm}$ height cores, with lengths ranging from $45 \mathrm{~cm}$ to $60 \mathrm{~cm}$. Here width refers to the direction parallel to the fracture plane. The cores were screened for the appearance of a stratification that ran uniformly along the length of the core. A single line of stratification was marked along both sides of a selected core with a pencil; this was the target area for fracturing.

2. A cold chisel was used to lightly imprint the core along the marked line. The core was placed on a hard, flat surface during this imprinting. Once a light imprint had been tapped along both sides, the chisel was applied with more force at any apparent weak point. Patience was required during this step, and eventually a small crack would appear under the persistent application of the chisel.

3. Once a small fracture had formed at one end, it was extended by application of the chisel just beyond the apex of the fractured portion, until the fracture extended along the length of the sample, and the two halves of the core were separated.

4. Care was taken in separating the two sample halves, as small portions of the core were loose along the surface of the fracture. The integrity of the fracture surface was maintained as much as possible.

5. The fracture halves were weighed, then put back together and held in place with a series of $\mathrm{C}$ clamps. A high viscosity epoxy was used to seal the edges of the fractures along the core length. One-sixteenth inch tube fittings were attached on the fracture at the ends of the core (Fig. 5.1), using the same epoxy.

6. When the fracture was sealed and the fittings were securely bonded to the core, the core was rolled in a hot, low-viscosity epoxy sealant, which slightly penetrated all the rock 
surfaces and completely sealed the core. The epoxy consisted of a 7/3 mixture by mass of resin/hardener that had been heated for approximately $10 \mathrm{~min}$ at $300 \mathrm{~F}$. The heating reduced the viscosity of the epoxy and increased the rate of cross-linking of the polymers. The epoxy hardened sufficiently in 48 hours for the core to be used.

\section{$\underline{\text { 5.3.3 Core Saturation }}$}

To emulate a NAPL contaminated fractured media, the fracture/matrix system was saturated with deionized water, and a residual saturation of NAPL was created in the fracture. The procedure was as follows:

1. The core was evacuated and flushed with carbon dioxide. The evacuation/flushing was repeated three times to remove as much of the air as possible. Any remaining gas after the final evacuation was predominantly carbon dioxide.

2. After a final evacuation, deaired and deionized water was introduced at a rate of 0.25 $\mathrm{mL} / \mathrm{min}$ until the core was saturated with water by imbibition. Any remaining small pockets of carbon dioxide should dissolve into the water, leaving a completely saturated system. The core was then capped and weighed.

3. A known volume of decane was pumped into the fracture from an injection loop. The fracture entry pressure for decane was less than $10 \mathrm{~cm}$ of water, and the maximum capillary pressure during primary drainage would not be expected to exceed $15 \mathrm{~cm}$ of water (Reitsma and Kueper, 1994). The matrix entry pressure for decane can be estimated from the permeability and porosity (assuming tortuosity is equal to unity) by

$$
P_{b}=\sigma \sqrt{\frac{\phi}{2 k}}
$$


where $\sigma$ is the interfacial tension between the decane and water. With $\sigma=25$ dyne $/ \mathrm{cm}, \phi$ $=0.20$, and $k=0.1 \mathrm{D}$, the estimated entry pressure is $25 \mathrm{kPa}$, or $255 \mathrm{~cm}$ water. Because the matrix entry pressure was an order of magnitude larger than the capillary pressure in the fracture, all of the decane remained in the fracture.

4. The decane injection was followed by water injection to create a residual decane saturation in the fracture. The waterflood flow rate was $2.0 \mathrm{~mL} / \mathrm{min}$, at least double the flow rate of the tracer experiments, to ensure that NAPL was not produced during the tracer experiments.

5. The amount of NAPL left in the fracture was quantified by the difference between the injected volume and the volume that was forced out during the subsequent waterflood. Measurement of the NAPL that was forced out was made by collection of the effluent in a volumetric flask of known weight. The amount of NAPL in the flask can be determined from the average density of the water and NAPL in the flask volume:

$$
V_{N f}=\frac{M_{T}-V_{T} \rho_{W}}{\left(\rho_{N}-\rho_{W}\right)}
$$

where

$$
\begin{aligned}
& V_{N f}=\text { NAPL volume in flask } \\
& M_{T}=\text { total liquid mass } \\
& V_{T}=\text { total liquid volume } \\
& \rho_{W}=\text { water density } \\
& \rho_{N}=\text { NAPL density }
\end{aligned}
$$


The volume of the NAPL in the core is the difference between the volume of NAPL in the flask and the injected volume of NAPL. The injected volume of NAPL was measured by mass, so the volume of NAPL in the core is given by

$$
V_{N c}=\frac{M_{N i}}{\rho_{N}}-\frac{M_{T}-V_{T} \rho_{W}}{\left(\rho_{N}-\rho_{W}\right)}
$$

where $M_{N i}$ is the mass of NAPL injected.

This estimate of $V_{N c}$ was an indirect measurement of a relatively small NAPL volume, which warranted an error analysis of the technique. A simple first-order second-moment analysis of equation (5.5) (assuming no correlation between the variables, i.e., the covariance matrix is zero) gives

$$
\begin{aligned}
\sigma_{V_{N}}^{2} & =\frac{f V_{N}}{f M_{N i}} \sqrt{ } \sigma_{M_{N i}}^{2}+\frac{f V_{N}}{f M_{T}} \sqrt{ } \sigma_{M_{T}}^{2}+\frac{f V_{N}}{f V_{T}} \sqrt{ } \sigma_{V_{T}}^{2} \\
& +\frac{f V_{N}}{f \rho_{N}} \sqrt{ } \sigma_{\rho_{N}}^{2}+\frac{f V_{N}}{f \rho_{w}} \sqrt{ } \sigma_{\rho_{w}}^{2}
\end{aligned}
$$

Substituting the differentiated terms yields

$$
\begin{aligned}
\sigma_{V_{N c}}^{2}= & \frac{-1}{\rho_{N}} \sqrt{ } \sigma_{M_{N i}}^{2}+\frac{1}{\rho_{w}-\rho_{N}} \sqrt[v]{ } \sigma_{M_{T}}^{2}+\frac{-\rho_{w}}{\rho_{w}-\rho_{N}} \sqrt{ } \sigma_{V_{T}}^{2} \\
& +-\frac{M_{N i}}{\rho_{N}^{2}}+\frac{-M_{T}+\rho_{w} V_{T}}{\left(\rho_{N}-\rho_{w}\right)^{2}} \sqrt{ } \sigma_{\rho_{N}}^{2}+\frac{M_{T}-\rho_{N} V_{T}}{\left(\rho_{N}-\rho_{w}\right)^{2}} V_{\lrcorner} \sigma_{\rho_{w}}^{2}
\end{aligned}
$$

Table 5.2 shows the results of this calculation made for experiment Frac11. The uncertainty in the mass was dependent on the balance used to measure the mass. The uncertainty in the total volume was that specified by the manufacturer of the $25 \mathrm{~mL}$ volumetric flask. The densities of water and decane were available in the literature to four significant figures at 22 degrees $\mathrm{C}$. Note that the largest contribution to the uncertainty was the measurement of the total volume. 


\subsubsection{Injection and Sampling of Tracers}

A schematic of the experimental setup for the tracer experiments is shown in Fig. 5.2. The procedure for injection and sampling of tracer is as follows:

1. Steady flow of water was established in the system. The flow rate was verified by mass measurement of the effluent over a given length of time.

2. The injection loop was switched to the load position, taking the loop off line. The tracer solution was then flushed through the loop. When the loop was full of the tracer solution, the switch was manually turned to the inject position, which put the loop back on line and pushed the tracer slug into the rock core.

3. Sampling occurred through a GC autosampler with a flow-through syringe. The column effluent was constantly flowing through the syringe - a pneumatic piston injected the contents of the syringe periodically onto the GC column where quantitative analysis occurred.

4. The sampling system, GC, and data acquisition were handled by a portable PC running Peaksimple for Windows software. The concentration data was then transferred via floppy to a desktop PC for data analysis.

\subsection{Modeling the Output Curves}

The 1-D fracture model presented in Section 4 was theoretically appropriate for modeling the results from these tracer experiments. However, because this model required a numerical inversion of the Laplace transform solution, it was not convenient for implementation in an optimization scheme. Tang et al. (1981) offers a similar model with a semi-analytical solution in normal space. Tang s model differs from the one presented in Section 4 in three important ways: 
1. One of the boundary conditions specifies a matrix that extends to infinity in the lateral direction. Because this condition results in an infinite mean residence time, the solution was not appropriate for the analysis given in Section 4. However, this condition did not effect the modeling of the laboratory results, since the tracer penetration depth in the lab was less than the width of the matrix (as shown in Section 5.6).

2. The Tang solution allowed only equilibrium partitioning between the tracer and NAPL, i.e., the inclusion of a retardation factor based on some linear exchange process. Again this limitation made the Tang model inappropriate for the analysis in Section 4 . However, the lab experiments were completed at flow rates that should have ensured equilibrium partitioning.

3. The Tang solution uses a constant inlet concentration boundary condition, instead of the Dirac pulse. To simulate a finite period of tracer injection, a second response curve is calculated according to a time lag defined by the duration of the tracer injection (Vermuelen, 1958). The second curve is then subtracted from the first to yield a composite curve that corresponds to the finite slug solution, i.e.,

$$
\begin{aligned}
& C_{\text {slug }}(t)=C_{\text {const. }}(t) \quad t<t_{s} \\
& C_{\text {slug }}(t)=C_{\text {const. }}(t)-C_{\text {const. }}\left(t-t_{s}\right) \quad t \geq t_{s}
\end{aligned}
$$

where $C_{\text {const }}(t)$ is the solution from the constant inlet concentration boundary condition and $t_{S}$ is the slug duration.

Figure 5.3 shows the coordinate system that is the basis for this model. Tang makes the following assumptions related to the geometry and hydraulic properties of the system:

1. The width of the fracture is much smaller than its length. 
2. Transverse diffusion and dispersion within the fracture assure complete mixing across the fracture width at all times.

3. The permeability of the porous matrix is very low and transport in the matrix will be by molecular diffusion.

In the application of Tang s model to the transport of partitioning tracers, the following additional assumptions are made, and reflected in the modification of the solution:

4. The tracers do not decay significantly.

5. There is no sorption of the tracers to the fracture wall or within the porous matrix.

The differential equation for tracer transport in the fracture is written

$$
\frac{f C_{f}}{f t}+\frac{u}{R} \frac{f C_{f}}{f x}-\frac{D_{L}}{R} \frac{f^{2} C_{f}}{f x^{2}}-\frac{\phi_{m} D_{m}}{b R} \frac{f C_{m}}{f y}=0
$$

where

$$
\begin{aligned}
C_{f} & =\text { concentration in the fracture } \\
C_{m} & =\text { concentration in the matrix } \\
u & =\text { average linear velocity in fracture } \\
R & =\text { retardation factor (equation (4.11)) } \\
2 b & =\text { fracture width } \\
D_{L} & =\text { longitudinal dispersion coefficient for the solute in the fracture } \\
D_{m} & =\text { diffusion coefficient for the solute in the matrix } \\
\phi_{m} & =\text { matrix porosity }
\end{aligned}
$$

The equation for the transport of the solute in the porous matrix can be written as

$$
\frac{f C_{m}}{f t}-D_{m} \frac{f^{2} C_{m}}{f x^{2}}=0 \quad h \leq x \leq x
$$


The boundary conditions for (5.10) are as follows:

$$
\begin{aligned}
& C_{f}(0, t)=C_{o} \\
& C_{f}(\times, t)=0 \\
& C_{f}(x, 0)=0
\end{aligned}
$$

where $C_{O}$ is the source concentration. The boundary conditions for (5.11) are

$$
\begin{aligned}
& C_{m}(b, x, t)=C_{f}(x, t) \\
& C_{m}(\times, x, t)=0 \\
& C_{m}(y, x, 0)=0
\end{aligned}
$$

Note that boundary condition (5.15) expresses the coupling of equation (5.10) for the fracture transport and equation (5.11) for the matrix transport. Tang uses a Laplace transform approach to derive the following solution for transport in the fracture:

$$
\frac{C_{f}}{C_{o}}=\frac{2 \exp (v x)^{\times}}{\pi^{1 / 2}} \exp -\varepsilon^{2}-\frac{v^{2} x^{2}}{4 \varepsilon^{2}} \operatorname{erfc} \frac{Y}{2 T} d \varepsilon
$$

where

$$
\begin{aligned}
l & =\frac{x-R}{2} \frac{-R}{D_{L} t} V^{1 / 2} \\
v & =\frac{u}{2 D_{L}} \\
Y & =\frac{x^{2} \phi_{m} D_{m}^{1 / 2}}{4 D_{L} b \varepsilon^{2}} \\
T & =\frac{R x^{2}}{t}-\frac{\sqrt{2}}{4 D_{L} \varepsilon^{2}} \sqrt{\downarrow}
\end{aligned}
$$


The solution can be written in terms of dimensionless variables and parameters, by redefining the terms, in a similar fashion as the procedures followed in Sections 3 and 4. For this case, we make the following definitions:

$$
\begin{aligned}
& t_{D}=\frac{t u}{L} \\
& x_{D}=\frac{x}{x_{L}} \\
& y_{D}=\frac{y}{b} \\
& C_{f, D}=\frac{C_{f}}{C_{o}} \\
& C_{m, D}=\frac{C_{m}}{C_{o}}
\end{aligned}
$$

Note that definition (5.25) differs from (4.10) because the infinite matrix does not allow scaling by fracture spacing. With definitions (5.23-5.27), the solution can be written in terms of four dimensionless parameters, $R, \phi_{m}, N_{P e}$, and $N_{M a}$ where

$$
\begin{aligned}
& P e=\frac{u x_{L}}{D_{L}} \\
& N_{M a}=\frac{D_{m} x_{L}}{u b^{2}}
\end{aligned}
$$

Note the similarity between the matrix number derived for this solution (5.29) and the matrix number derived for the model of Section 4. Again, the difference lies in the scaling in the $y$ direction, where the Tang solution is scaled by the fracture width since the fracture spacing is infinite.

The solution (5.18) in terms of dimensionless variables and parameters is 


$$
\begin{aligned}
& C_{f, D}=\frac{2}{\sqrt{\pi}} \exp \frac{N_{P e}}{2} \sqrt{ }
\end{aligned}
$$

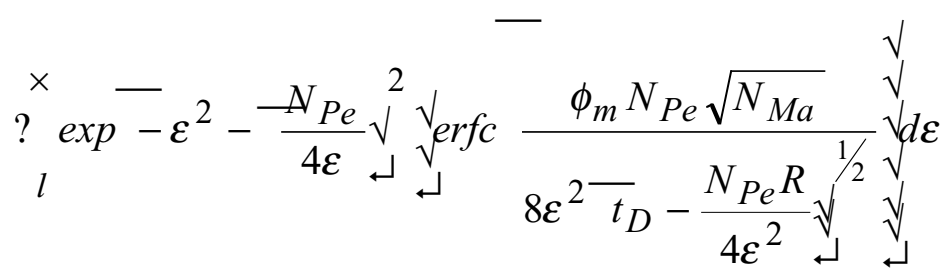

where

$$
l=\frac{R N_{P e}}{4 t_{D}} y^{1 / 2}
$$

Equation (5.30) is the solution that was used to model the tracer response data from the laboratory experiments.

A numerical routine was developed to evaluate the indefinite integral in (5.30). The goal of the routine was to integrate the solution into an Excel spreadsheet, so that the built-in solver could be used to fit the model to the experimental data. A front-end was developed in Visual Basic for Applications (VBA), the native language used in Microsoft Excel 97. The VBA frontend handled the interaction with the spreadsheet, and was dynamically linked to an optimized Fortran code, which solved the indefinite integral using a quadrature application of the trapezoid rule. The use of the optimized Fortran code compared to running the entire algorithm in VBA increased the speed by roughly 10 times, allowing the solver to work more efficiently.

\subsection{Curve Fitting}

In order to compare the model to experimental data, there should be a method to determine the best values for the input parameters. Ideally, the parameters could be determined independently, i.e., found in the literature or estimated with some accuracy. These parameters could then be used as input to forward simulations that could be compared to the experimental results. 
However, the necessary inputs were not known in this case. Instead, the approach used here was to fit the model to the experimental data, then use the best fit parameters to estimate the unknown experimental parameters. The following section explains the approach in terms of the actual variables.

We begin with a summary of what model parameters and variables are known and unknown for the experiments.

Known:

$x_{L} \quad$ column length

w column width, parallel to fracture

$q \quad$ flow rate

$D_{m} \quad$ diffusion coefficient in matrix

$\phi_{m} \quad$ matrix porosity

Unknown:

$b \quad$ half fracture width

$D_{L} \quad$ dispersion coefficient

Note that other unknown variables, such as velocity in the fracture, can be calculated from combinations of the above.

Equation (5.30) contains two parameter groupings $\left(\phi_{m}\left(N_{M a}\right)^{0.5}, N_{P e}\right)$ and one independent variable $\left(t_{D}\right)$. The dimensionless time can be written in terms of the experimental flow rate and $N_{M a}$. First, the velocity in the fracture is written in terms of the flow rate, the fracture width and the width of the core parallel to the fracture:

$$
u=\frac{q}{2 b w}
$$


Equation (5.32) is combined with equations (5.23) and (5.29) to give

$$
t_{D}=\frac{t q^{2} N_{M a}}{4 w^{2} D_{m} x_{L}}
$$

With equation (5.33), the model curve can be fit to the experimental concentration data using the actual experimental time $(t)$, and the two parameter groups, $\phi_{m}\left(N_{M a}\right)^{0.5}$ and $N_{P e}$. After $N_{M a}$ and $N_{P e}$ are estimated from the curve fit, the fracture width can be calculated from $N_{M a}$, the velocity can then be determined from (5.32), and $D_{L}$ can be calculated from the velocity and $N_{P e}$.

The model curves were fit to the experimental data by minimizing the sum of the absolute value of the residuals (objective function) with the built in Excel solver:

$$
S R=\frac{1}{n}{ }_{i=1}^{n} \frac{\left|C_{i, \text { exp }}-C_{i, \text { mod el }}\right|}{C_{i, \text { mod el }}}
$$

where $n$ is the number of data points. Figure 5.4 shows an example of the model curve fit to the methanol data from experiment Frac5. The fit is poor between the model curve and the experimental data. The sum of the residuals is 1.2. One possible explanation for the poor fit is that the model matrix porosity should be different from the measured average porosity of the entire core.

The model porosity may differ from the measured average porosity for several reasons. The areal porosity in the immediate vicinity of the fracture may be slightly different than the average volumetric porosity for the entire core. Also, the roughness of the fracture causes nonuniform tracer flow. So the areal matrix porosity encountered by the tracer along the flow path may be slightly higher or lower than the measured average porosity for the entire core.

Figure 5.5 shows the fit for the same experimental data as shown in Fig. 5.4, where the model matrix porosity was allowed to vary from the measured value to improve the fit. Note that 
the fit is visually better, and the sum of residuals decreased to 0.085 from the previous 1.2 . The effective matrix porosity was 0.17 for this model result, about $10 \%$ less than the measured value of 0.19. Because the curve fit was dramatically improved and the decrease in the effective matrix porosity was small and theoretically defensible, the porosity was allowed to vary during the curve fitting routine for all of the results presented in this section. All of the fit effective porosity values are within $10 \%$ of the measured value of 0.19 .

\subsection{Results for Single Fracture Experiments, No NAPL}

\section{$\underline{\text { 5.6.1 Experiment Frac5 - Initial Study of Matrix Diffusion }}$}

The Frac5 experiment was a single-fracture test in Berea sandstone using methanol, IPA, 1propanol, and hexanol as tracers. The goal of this experiment was to determine some of the transport characteristics of the alcohol tracers in a fracture, without any NAPL present. The experiment was intended to provide baseline data for comparison to future tracer experiments where NAPL was present in the fracture.

Table 5.3 shows the relevant column characteristics for this experiment. Note that the diffusion coefficients in the matrix were calculated by multiplying the bulk water diffusion coefficients by the tortuosity of 0.4 (Donaldson et al., 1976). Figure 5.6 shows the response data for the four tracers. The concentrations were normalized by dividing the measured output concentrations by the injected tracer concentrations. Two important trends are evident in this Fig. 5.6. First, the response data does not show exponential decline as is typical of porous media tracer response data, i.e., the tails are not linear on the semi-log plot. Second, the tailing of the response data appears to increase with increasing diffusion coefficient, i.e., the methanol tails the most, and the hexanol tails the least. However, the hexanol response is the only data that shows 
a clear difference from the other tracers. The methanol data does appear to fall slightly above the rest, as would be predicted for the tracer with the highest diffusion coefficient.

When the model was fit to the output curves, a global fit was completed (i.e., all four tracer output curves were fit simultaneously). The fit parameters that should be the same for every tracer were kept at the same value for all four tracers, i.e.,

$$
\mathrm{NPe}, \text { methanol }=\mathrm{NPe} \text { IPA }=\text { NPe, } \text { propanol }=\mathrm{NPe}, \text { hexanol }
$$

The matrix number, $N_{M a}$, was scaled for each tracer by its diffusion coefficient in water (Table 5.1), e.g.,

$$
N_{\text {Ma,IPA }}=\frac{D_{m, I P A}}{D_{m, \text { methanol }}} N_{\text {Ma,methanol }}
$$

with the matrix number for 1-propanol and hexanol scaled the same way.

Table 5.3 shows the parameters generated from the fitting process, and the column characteristics calculated from these estimated parameters. The Peclet number predicted from the fits was 115 , which is high for laboratory porous media tracer experiments, but is not atypical for single-fracture experiments. Maloszewski and Zuber $(1990,1993)$ report values for $N_{P e}$ ranging from 50 to 100 , using a similar model. The fracture width calculated from $N_{M a}$ is $0.0180 \mathrm{~cm}$, a reasonable value for a fracture created without any spacers holding the two rock halves apart.

Figures 5.7-5.10 show the model curves fit to the response data for each of the tracers. The sum of residuals (SR from equation 5.34) was lowest for the propanol tracers, and the goodness of fit for these two data sets $(\mathrm{SR}=0.042,0.062)$ can be verified visually in Figs. 5.7 and 5.8. The methanol and hexanol model fits are shown on the same page (Figs. 5.9 and 5.10) to visually evaluate the ability of the model to account for differences in matrix diffusion. The 
model fit the methanol data well over the entire duration of the data. However, the model did not fit the hexanol data as well for late time, after approximately 11 fracture pore volumes. The model predicted that there should be more tailing at late time than the experimental data indicates. The error in the concentration data increases as the detection limit of the GC is approached, which partially explains this discrepancy. The scaling of the matrix number by the diffusion coefficient of the tracers allowed the model to account for the differences in response data.

The mass recovery data in Table 5.3 shows that the relative recovery for the tracers decreased with increasing diffusion coefficient. The best mass recovery was $80 \%$ for hexanol. To help explain the poor mass recovery, the concentration distribution of IPA was calculated from the model. Figures $5.11-5.16$ show these computed matrix concentration contours for IPA at progressing times during the simulation. The concentrations given by the contours were normalized by dividing by the injected concentration. After the tracer slug has passed through the fracture, a significant amount of tracer remains in the first few millimeters of the matrix. Figure 5.17 shows a plot of the mass history for this simulation. The mass in the matrix comprises almost $30 \%$ of the total tracer mass at approximately $10 \mathrm{~mL}$ produced volume. After the mass of tracer in the fracture has decreased to nearly zero, the mass in the matrix continues to comprise approximately $20 \%$ of the total injected mass, which explains the $75-80 \%$ mass recoveries seen in the experimental results.

\subsubsection{Experiment Frac6: Verification of Matrix Diffusion Effect}

The Frac6 experiment was a single-fracture test in clean Berea using IPA, hexanol, tritiated water $\left(\mathrm{T}_{2} \mathrm{O}\right)$, and radiolabeled chloride $\left({ }^{36} \mathrm{Cl}^{-}\right)$. Although the increased retardation and tailing of the lighter tracers evident in experiment Frac 5 could be explained in terms of a matrix diffusion 
process, additional studies were warranted to verify this explanation. The inclusion of the inorganic tracers was intended to provide data that would help eliminate other possible explanations for the tailing effect. We considered three general processes that might cause the tailing or retardation in the response data—matrix diffusion, sorption, and exchange at liquid/gas interfaces.

Matrix diffusion is the penetration of solutes from the flowing part of the fracture into non-flowing regions, either on the surface of the fracture or in the rock matrix itself. The farther a dissolved constituent penetrates into these immobile regions, the more retardation should be evident in the response curve. Therefore, the retardation would be expected to increase with increasing diffusion coefficient (Table 5.1) so that tritiated water would be retarded the most and hexanol the least.

Sorption for these solutes can be divided into two possible areas--sorption to surface organic constituents and surface ion-exchange. For sorption to organic surface constituents, the more organic, non-polar molecules like hexanol would be expected to sorb the most, with inorganics like $\mathrm{Cl}^{36}$ and $\mathrm{T}_{2} \mathrm{O}$ sorbing the least.

The effect of surface ion-exchange is more difficult to state per se. Edgar (1997) indicates that in certain soils, the exchange of ${ }^{3} \mathrm{H}$ with connate water can cause tritium to lag relative to IPA. However, this effect will vary with the type of solid matrix through which the flow occurs. Because the hydronium ion is positive, it is unlikely that the same effect would be seen with respect to the negatively charged chloride ion. The IPA and hexanol should be unaffected by the ion-exchange process.

Exchange at liquid/gas interfaces is the process where a solute transfers into trapped gas phases present in the core. Although the core was prepared carefully to avoid the presence of gas 
phases, the possibility exists that not all of the gas was removed. For this process, the most volatile chemical (IPA) would be retarded the most, and $\mathrm{Cl}^{36}$ would be retarded the least.

Figure 5.18 shows the response data for the four tracers in this experiment. The retardation and tailing of the tracers was quantified by calculating the cumulative mean residence time of the tracers (equation 2.2). The trends are also evident visually in Fig. 5.18. At late time, the concentration of the hexanol has decreased the most, and the concentration of the tritiated water has decreased the least. Table 5.4 summarizes the predicted retardation trends for each process, along with the actual trend demonstrated by the experiment. The expected trend for matrix diffusion is the same as the experimental results, so this experiment gives strong evidence that matrix diffusion is the major factor causing the retardation of the lighter, more diffusive solutes with respect to the heavier ones.

Table 5.5 shows the parameters generated from the fitting process for the Frac6 data, and the column characteristics calculated from these estimated parameters. Note that the best fit parameters for Frac6 vary slightly from those of Frac5. These experiments were completed using the same column at the same flow rate. However, between the two experiments, some difference exists in the velocity profile within the fracture. The difference is evident when the IPA response data is plotted for both experiments, as shown in Fig. 5.19. The IPA response data for Frac5 breaks through slightly earlier, and tails slightly less than the IPA response data for Frac6. Some error in the flow rate might have occurred for one of the experiments, or because the flow in the column was stopped completely between the two experiments, a slightly different flow regime may have been established in the second experiment.

The aperture width calculated from the fit parameters for experiment Frac6 was 0.024 $\mathrm{cm}$, which was an increase of $0.006 \mathrm{~cm}$ from Frac5. The Peclet number and the effective matrix 
porosity were the same for both experiments. The sum of residual values for Frac6 ranged from 0.10 to 0.18 . Figures 5.20 to 5.23 show the individual model curve fits for the tracer response data. The fit for the hexanol response data for Frac6 was similar to the hexanol fit for Frac5, where there is some deviation of the model from the late time experimental data. Figure 5.21 shows more tailing in the model curve than is evident in the experimental hexanol response data of Frac6.

\subsubsection{Experiment Frac8: Rate Dependence of Matrix Diffusion}

The Frac8 experiment was a single-fracture test in clean Berea using IPA and hexanol as tracers. Frac8 was carried out at a higher flow rate than the previous experiments. Because matrix diffusion is a kinetic limited process, Frac 8 was intended to verify this rate limitation. Time is required for a solute to diffuse transverse to flow into the dead regions along the fracture surface and in the matrix itself, so the shorter the residence time of the tracer, the less the retardation and tailing effects should be evident. The flow rate was increased from $0.17 \mathrm{~mL} / \mathrm{min}$ for Frac5 and Frac6 to $1.08 \mathrm{~mL} / \mathrm{min}$ for Frac8.

Figure 5.24 shows the IPA and hexanol response data for experiment Frac8. Note that very little difference can be seen between the IPA and hexanol curves. The increased velocity in the fracture decreased the impact of matrix diffusion on the tracer response data, so the higher diffusion coefficient of the IPA had little effect in comparison to hexanol. Section 4.2.1 describes this effect in terms of a decreasing matrix number. As shown in Table 5.6, the matrix number was less for this experiment compared to Frac6.

Figures 5.25 and 5.26 show a comparison between the tracer response data for Frac6 and Frac8, on an effluent volume basis. The Frac6 response data are retarded slightly compared to 
the Frac 8 response data. The Frac6 response data also show significantly increased tailing compared to the Frac8 response data.

Table 5.6 shows the parameters generated from the fitting process for the Frac 8 data, and the column characteristics calculated from these estimated parameters. Figures 5.27 and 5.28 show the actual curve fits for the two tracers. The matrix number for Frac 8 decreased compared to Frac6, as would be expected for the higher velocity experiment (equation 5.29). The fit matrix numbers for IPA and hexanol in Frac8 decreased by a factor of 9.1, while the flow rate had been decreased by a factor of 6.4. The discrepancy is likely due to a different flow regime in the fracture for Frac8, which causes a variation in the mass balance tracer fracture aperture.

With a significant increase in the flow rate, some of the immobile regions along the fracture surface would be mobilized, increasing the tracer fracture aperture. The velocity in the fracture varies inversely with the fracture aperture (equation 5.32). The matrix number varies with the inverse square of the fracture aperture (equation 5.29). If we assume that the tracer fracture aperture is some positively increasing function of the flow rate, i.e.,

$$
b=q^{a} \quad a>0
$$

then equations (5.29), (5.32) and (5.37) can be combined to give

$$
N_{M a}=\frac{2 w D_{m} x_{L}}{q^{1+a}}
$$

Equation (5.38) shows that if the effective tracer fracture aperture increases with increasing flow rate, due to the mobilization of some dead regions along the fracture surface, then the matrix number will decrease by a factor greater than the flow rate. This trend is seen in experiments Frac6 and Frac8. 
The longitudinal dispersion in the fracture was estimated from the Peclet numbers for Frac6 and Frac8. The longitudinal dispersivity, $D_{L}\left(\mathrm{~cm}^{2} / \mathrm{s}\right)$, is often quantified in porous media flow as

$$
D_{L}=\alpha u^{n}+\tau D_{w}
$$

where

$$
\begin{aligned}
& \alpha=\text { the dispersivity of the medium } \\
& n=\text { the dispersivity exponent } \\
& \tau=\text { tortuosity of the porous medium }
\end{aligned}
$$

and the units of the dispersivity are dictated by the value of the exponent. In this research, we assume that the dispersion dominates bulk diffusion in the longitudinal direction in the fracture, a valid assumption at Peclet numbers greater than about 5. While $n$ is about 1.2 for flow in porous media flow, Dronfield and Silliman (1993) show that the value of the exponent falls closer to 2 than 1 for fractures that were tested at varying degrees of wall roughness. From equations (5.28) and (5.39), we can solve for the dispersivity exponent

$$
n=1-\frac{\ln \left(\frac{P e_{1}}{P e_{2}}\right)}{\ln \left(\frac{u_{1}}{u_{2}}\right)}
$$

After the exponent is calculated, it can be substituted into (5.37) to give the dispersivity. Table 5.6 shows the calculated dispersivity and dispersivity exponent using the Peclet number and velocity from experiments Frac6 and Frac8. The exponent was 2.5, which is slightly higher than the range of $1.7-2.44$ measured by Dronfield and Silliman (1993). The dispersivity was calculated from equation (5.39) to be $100\left(\mathrm{~s}^{1.5} / \mathrm{cm}^{0.5}\right)$. 


\subsection{Results for Single Fracture Experiments With NAPL}

\subsubsection{Frac11: Low Flow Rate Experiment}

The Frac11 experiment was a single-fracture partitioning tracer test in Berea sandstone, with residual decane. The conservative tracer was IPA, and hexanol and heptanol were the partitioning tracers. $1.8 \pm 0.4 \mathrm{~mL}$ decane was left in the column after the waterflood. This experiment was conducted in a different single-fracture core than were the previous experiments. The epoxy on the previous core was degraded due to the attempted use of a chlorinated solvent as a NAPL. The new core was cut from the same rock sample and was fractured in the same fashion as the previous core.

The partition coefficients were measured (section 5.3.1) between decane and the two partitioning tracers before the column experiment was conducted. Table 5.7 gives the results, calculated from equation (5.2). The partition coefficients were $5.6 \pm 0.3$ and $22.4 \pm 0.7$ for hexanol and heptanol respectively, between water and decane.

Establishing the proper flow rate for this experiment required the consideration of two factors. As discussed in Section 4.3, matrix diffusion can have a significant effect on tracer transport at low flow rates, while higher flow rates can lead to non-equilibrium between the partitioning tracers and the resident NAPL. No correlations exist for calculating partitioning tracer water-NAPL mass transfer coefficients in a fracture, so the experiment was simply designed so that the partitioning tracers would have a mean residence time of 2-3 hours, adequate to achieve equilibrium in a typical porous media column experiment (Section 3.4.3). Assuming a fracture aperture of approximately $0.02 \mathrm{~cm}$, a flow rate of $3.3 \times 10^{-4} \mathrm{~mL} / \mathrm{s}$ would give a fracture mean residence time of 5 hours. 
Table 5.8 shows the relevant column characteristics for this experiment. Figure 5.29 shows the response data for the three tracers. Note that both matrix diffusion (tailing of IPA relative to others) and partitioning (later breakthrough and lower peaks of partitioning tracers compared to IPA) are demonstrated in the data. The cumulative mean residence volume of each of the tracers was calculated from equation 2.2 and is shown in Fig. 5.30. The figure shows that the mean residence volumes of the partitioning tracers start out increasing faster than the mean residence volume of the IPA, but the diffusive tailing of the IPA concentration response causes a late time reversal of this trend in the cumulative mean residence volumes.

The volume of decane in the fracture was calculated from equation (2.9) using the mean residence volumes (MRVs) of the tracers. The MRV for hexanol was actually less than that of the IPA at late time, which would result in the calculation of a negative NAPL volume. The heptanol data gave a positive estimate of the NAPL volume $(0.3 \mathrm{~mL})$, but this estimate was $83 \%$ less than the known value of $1.8 \mathrm{~mL}$. Because the hexanol response data showed less diffusive tailing than the IPA data, a comparison between the MRVs of the two partitioning tracers was also used to estimate the volume of NAPL in the column using equation (2.9). The result was $0.5 \mathrm{~mL}$ decane, which was $72 \%$ less than the measured value.

Robinson and Tester (1984) have suggested that extrapolation of the early response curve could help eliminate the effect of the late time tailing. While extrapolation of late time partitioning tracer data in porous media is a common practice when data are incomplete (i.e., Deeds et al. 1999), this is not the same as extrapolating early time data in fractured media. First, tracer transport in porous media is theoretically predicted to yield data that declines exponentially at late time, while tracer transport in a fracture does not theoretically yield exponentially declining data, especially early in the response curve (Becker, 1996). Secondly, 
for the case shown in Deeds et al. (1999), small errors in the late time data extrapolation would not lead to a significant increase in the overall uncertainly of the result. For the case being considered here, extrapolation of early time data will have a large effect on the result, as shown below.

A simple analysis was carried out to determine the relative impact of small errors in the slope of the extrapolated line on the overall NAPL volume prediction. Figures 5.31 and 5.32 show several extrapolation lines for the hexanol response. The slopes were varied enough so that the change was visually detectable. The extrapolation analysis was started at two different produced volumes, to determine the sensitivity to the starting point. Table 5.9 shows the results of this analysis. Note that for a given change in the slope of the extrapolation line, the MRT changes by a factor of approximately $2 / 3$ for this case. For this case with low NAPL volumes, the sensitivity to small changes in the slope causes very significant changes in the estimated volume. The extrapolation of early time data was a poor technique for analyzing this set of tracer data.

An attempt was made to fit the model to the tracer concentration data. The IPA concentration data were fit in the same manner as the previous experiments. The modeled decane saturation in the fracture was then varied to minimize SR for the two partitioning tracers. While the model fit the IPA concentration data well (Fig. 5.33), the fit was poor for the concentration data of the two partitioning tracers (Figs. 5.34-5.35), with an SR of 0.24 and 0.75 for hexanol and heptanol, respectively. The modeled NAPL volume was $0.34 \mathrm{~mL}, 81 \%$ less than the known value of $1.8 \mathrm{~mL}$.

Because the model fit to the concentration data was poor, an attempt was made to model the tracer cumulative MRV data. Equation 5.34 was used, with the cumulative MRV data in 
place of concentration. The results of this fit are shown in Table 5.8. The SRs were all less than 0.033, at least an order of magnitude better than the concentration fits. The IPA MRV data was fit first, then the parameters were held constant, and the NAPL saturation varied to fit the MRV data for the partitioning tracers. Figures 5.36-5.38 show the MRV experimental data and model fits for each of the tracers. The fit NAPL volume was $1.0 \mathrm{~mL}$, the closest result to the measured volume of any of the techniques described in this section.

\subsubsection{Frac12: High Flow Rate Experiment}

The Frac12 experiment was a single-fracture test in Berea sandstone at waterflood residual decane using IPA as the conservative tracer with hexanol and heptanol as partitioning tracers. The flow rate for this experiment was approximately an order of magnitude higher than the previous one, at $0.217 \mathrm{~mL} / \mathrm{min}$ compared to $0.020 \mathrm{~mL} / \mathrm{min}$. The goal was to decrease the significance of matrix diffusion and perhaps improve the estimate of NAPL volume from the partitioning tracer data.

Table 5.10 gives the relevant physical properties for this experiment. Figure 5.39 shows the concentration response data for the tracers. Note that matrix diffusion is less evident in these response data, compared to experiment Frac11. However, Fig. 5.40 shows that the IPA MRV

still increases faster towards the end of the experiment than does the MRV of the partitioning tracers.

The inverse fitting of the model was applied again to the cumulative MRV data. As shown in Table 5.10, the SRs were 0.05 or less for the model fits. The calculated fracture aperture was $0.036 \mathrm{~cm}$, which compares favorably to the result from Frac11, which was 0.033 $\mathrm{cm}$. The MRV data and corresponding model fit curve are shown for each tracer in Figs. 5.415.43. The fitted NAPL saturation was 0.095 , which is $7 \%$ higher than the saturation estimate 
from Frac11, the lower flow rate experiment. The NAPL volume calculated from the MRV fits was $1.1 \mathrm{~mL}$, which is $16 \%$ higher than the Frac11 result, and $38 \%$ lower than the measured value. The difference in the percent increase for the saturation and the volume predictions is due to the slightly larger fracture aperture calculated from the fit parameters for Frac12. In general, the partitioning tracer data from the higher flow rate experiment provided a better estimate of the NAPL volume.

\subsection{Summary and Conclusions}

Laboratory partitioning tracer experiments were completed in single fracture Berea sandstone cores, both with and without NAPL present. The tracer response data in cores without NAPL showed retardation and late time tailing. Results from an experiment with both inorganic and organic tracers of varying diffusion coefficients provided strong evidence that the retardation and late time tailing were caused by matrix diffusion. The higher the diffusion coefficient of the tracer, the more pronounced was the impact of matrix diffusion. A semi-analytical single fracture model was fit to the concentration response data in order to estimate some experimental parameters, such as average fracture aperture and average flow velocity. In general, the model fit the data well, with the worst fit occurring for the tracers with the lowest diffusion coefficients.

The model was used to simulate the tracer concentration in the matrix of IPA for one of the experiments. The simulation results showed that the tracer penetrated approximately 2 millimeters into the matrix, and that a maximum of $30 \%$ of the total tracer mass was in the matrix during the course of the experiment, much of which remained even after the fracture concentration went to zero.

Two experiments with varying flow rates verified the kinetic limitations that characterize matrix diffusion effects. The higher the average velocity of the tracer in the fracture, the less 
pronounced the effects of diffusion. Results from these experiments were also used to predict the dispersivity and dispersivity exponent for the fracture. The dispersivity exponent, at 2.5 , was higher than expected, but within the range of published values for single fracture experiments.

Two experiments were completed with NAPL in the fracture at two different flow rates. The partitioning tracer response curves showed both the effects of partitioning (retardation and lower peaks with respect to the conservative tracer) and matrix diffusion (more pronounced late time tailing of the lighter conservative tracer). Due to matrix diffusion, the mean residence volume of the conservative tracer increased faster than that of the partitioning tracers at late time, which caused a poor estimate of NAPL volume in the fracture compared to the measured value. An analysis of early time data extrapolation showed that the results from the technique were too sensitive to small errors in the estimated slope to yield good results in this case.

The semi-analytical fracture model fit the conservative tracer concentration data well, but fit the partitioning tracer concentration data poorly. The model was therefore not suitable for predicting concentration histories for partitioning tracer in fractured cores. However, the model did provide a good fit for the tracer MRV data. The NAPL volume calculated from the fit parameters for the higher flow rate experiment was closer to the measured value than the volume calculated from the lower flow rate experiment, but the calculated volume for the higher flow rate experiment was still $38 \%$ lower than the measured value.

Fitting the semi-analytical model to the MRV tracer data was the most accurate method for estimating the NAPL volume in the fracture. Partitioning tracer tests in fractured rocks of high tortuosity ( 0.4 in this case) can significantly underestimate NAPL volumes if interpreted with standard method of moment equations. Inverse modeling of the partitioning tracer response data with a simulator that accounts for matrix diffusion may provide a more accurate estimate. 


\subsection{Tables}

Table 5.1: Physical Properties of Tracer Compounds

\begin{tabular}{|c|c|c|c|c|}
\hline Compound & Formula & $\begin{array}{c}\text { Molecular } \\
\text { Mass } \\
(\mathrm{g} / \mathrm{mol})\end{array}$ & $\begin{array}{c}\text { Solubility } \\
\text { in Water } \\
(\mathrm{mg} / \mathrm{L})\end{array}$ & $\begin{array}{c}\text { Diffusion Coeff. } \\
\text { in Water, } 25^{\circ} \mathrm{C} \\
\left(\mathrm{cm}^{2} / \mathrm{s}\right)\end{array}$ \\
\hline methanol & $\mathrm{CH}_{4} \mathrm{O}$ & 32 & miscible & $1.50 \mathrm{E}-05$ \\
\hline 1-propanol & $\mathrm{C}_{3} \mathrm{H}_{8} \mathrm{O}$ & 60 & $>10000$ & $1.02 \mathrm{E}-05$ \\
\hline isopropanol & $\mathrm{C}_{3} \mathrm{H}_{8} \mathrm{O}$ & 60 & miscible & $9.90 \mathrm{E}-06$ \\
\hline 1-hexanol & $\mathrm{C}_{6} \mathrm{H}_{14} \mathrm{O}$ & 102 & 3000 & 7.76E-06 \\
\hline 1-heptanol & $\mathrm{C}_{7} \mathrm{H}_{16} \mathrm{O}$ & 116 & 1000 & 7.07E-06 \\
\hline 1-octanol & $\mathrm{C}_{8} \mathrm{H}_{18} \mathrm{O}$ & 130 & 200 & $6.44 \mathrm{E}-06$ \\
\hline tritiated water & $\mathrm{T}_{2} \mathrm{O}$ & 20 & miscible & $2.26 \mathrm{E}-05$ \\
\hline chloride & - & 36 & $\begin{array}{c}320000 \\
\text { (as } \mathrm{NaCl} \text { ) }\end{array}$ & $1.90 \mathrm{E}-05$ \\
\hline
\end{tabular}

Table 5.2: Results of Uncertainty Analysis for Residual NAPL Volume

\begin{tabular}{|lllll|}
\hline \multicolumn{1}{|c}{ Variable } & \multicolumn{1}{c}{ Value } & \multicolumn{1}{c}{$\sigma$} & $\begin{array}{c}\text { variance } \\
\text { contribution }\end{array}$ & $\begin{array}{c}\text { \% variance } \\
\text { contribution }\end{array}$ \\
\hline$M_{N i}(\mathrm{~g})$ & 5.840 & 0.002 & $7.51 \mathrm{E}-06$ & $5.02 \mathrm{E}-03$ \\
$M_{T}(\mathrm{~g})$ & 23.27 & 0.02 & $5.58 \mathrm{E}-03$ & $3.73 \mathrm{E}+00$ \\
$V_{T}(\mathrm{~mL})$ & 25.0 & 0.1 & $1.39 \mathrm{E}-01$ & $9.29 \mathrm{E}+01$ \\
$\rho_{N}(\mathrm{~g} / \mathrm{mL})$ & 0.730 & 0.001 & $8.19 \mathrm{E}-05$ & $5.48 \mathrm{E}-02$ \\
$\rho_{w}(\mathrm{~g} / \mathrm{mL})$ & 0.998 & 0.001 & $4.91 \mathrm{E}-03$ & $3.28 \mathrm{E}+00$ \\
$V_{N \boldsymbol{c}}(\mathbf{m L})$ & $\mathbf{1 . 8}$ & $\mathbf{0 . 4}$ & & \\
\hline
\end{tabular}


Table 5.3: Experiment Characteristics and Results for Frac5

\begin{tabular}{|c|c|c|c|c|c|}
\hline \multicolumn{6}{|c|}{ Known or Measured Parameters } \\
\hline Rock Type & & Berea & & & \\
\hline Column Length, $x_{L}$ & $\mathrm{~cm}$ & 45.9 & & & \\
\hline Column Width, $w$ & $\mathrm{~cm}$ & 4.9 & & & \\
\hline Flow Rate, $q$ & $\mathrm{~mL} / \mathrm{s}$ & 0.00283 & & & \\
\hline Slug Size, $t_{s}$ & $\mathrm{~mL}$ & 3.27 & & & \\
\hline Tracer & & Methanol & Propanol & IPA & Hexanol \\
\hline Eff. Diff. Coeff, $D_{m}$ & $\mathrm{~cm}^{2} / \mathrm{s}$ & $6.00 \mathrm{E}-06$ & $4.08 \mathrm{E}-06$ & $3.96 \mathrm{E}-06$ & $3.10 \mathrm{E}-06$ \\
\hline Injected conc. & $\mathrm{mg} / \mathrm{L}$ & 2026 & 2004 & 2006 & 1000 \\
\hline Recovery Percent & $\%$ & 74.2 & 76.4 & 77.1 & 80.0 \\
\hline \multicolumn{6}{|c|}{ Fit Parameters } \\
\hline Sum of Residuals, SR & -- & $8.48 \mathrm{E}-02$ & $6.28 \mathrm{E}-02$ & $4.78 \mathrm{E}-02$ & $1.10 \mathrm{E}-01$ \\
\hline Peclet number, $N_{P e}$ & -- & 115 & 115 & 115 & 115 \\
\hline Matrix number, $N_{M a}$ & -- & 25.3 & 17.2 & 16.7 & 13.1 \\
\hline Matrix porosity, $\phi_{m}$ & -- & 0.17 & 0.17 & 0.17 & 0.17 \\
\hline \multicolumn{6}{|c|}{ Parameters Calculated from Fit and Known Parameters } \\
\hline Velocity, $u$ & $\mathrm{~cm} / \mathrm{s}$ & 0.031 & & & \\
\hline Fracture Width, $2 b$ & $\mathrm{~cm}$ & 0.019 & & & \\
\hline Disp. Coeff., $D_{L}$ & $\mathrm{~cm}^{2} / \mathrm{s}$ & 0.012 & & & \\
\hline Fracture Volume & $\mathrm{mL}$ & 4.2 & & & \\
\hline Fracture MRT & $\mathrm{s}$ & 1495 & & & \\
\hline
\end{tabular}

Table 5.4: Expected and Measured Results for Possible Retardation Processes

\begin{tabular}{|l|llll|}
\hline Process & \multicolumn{4}{l|}{ Increasing Retardation --------------> } \\
\hline Matrix Diffusion & $\mathrm{Hexanol}$ & $\mathrm{IPA}$ & $\mathrm{Cl}^{36}$ & $\mathrm{~T}_{2} \mathrm{O}$ \\
Organic Sorption & $\mathrm{Cl}^{36}$ & $\mathrm{~T}_{2} \mathrm{O}$ & $\mathrm{IPA}$ & $\mathrm{Hexanol}$ \\
Hydronium Exchange & $\mathrm{Cl}^{36}$ & Hexanol & $\mathrm{IPA}$ & $\mathrm{T}_{2} \mathrm{O}$ \\
Anion Exchange & $\mathrm{Hexanol}^{36}$ & IPA & $\mathrm{T}_{2} \mathrm{O}$ & $\mathrm{Cl}^{36}$ \\
Liquid/gas Exchange & $\mathrm{Cl}^{36}$ & Hexanol & $\mathrm{T}_{2} \mathrm{O}$ & $\mathrm{IPA}^{36}$ \\
\hline Experimental Result & $\mathrm{Hexanol}^{36}$ & IPA & $\mathrm{Cl}^{36}$ & $\mathrm{~T}_{2} \mathrm{O}$ \\
Mean Res. Vol. (mL) & 23.3 & 25.8 & 29.1 & 32.1 \\
\hline
\end{tabular}


Table 5.5: Experiment Characteristics and Results for Frac6

\begin{tabular}{|c|c|c|c|c|c|}
\hline \multicolumn{6}{|c|}{ Known or Measured Parameters } \\
\hline Rock Type & & Berea & & & \\
\hline Column Length, $x_{L}$ & $\mathrm{~cm}$ & 45.9 & & & \\
\hline Column Width, $w$ & $\mathrm{~cm}$ & 4.9 & & & \\
\hline Flow Rate, $q$ & $\mathrm{~mL} / \mathrm{s}$ & 0.00283 & & & \\
\hline Slug Size, $t_{s}$ & $\mathrm{~mL}$ & 3.27 & & & \\
\hline Tracer & & IPA & Hexanol & $\mathrm{Cl}^{36}$ & $\mathbf{T}_{2} \mathrm{O}$ \\
\hline Eff. Diff. Coeff, $D_{m}$ & $\mathrm{~cm}^{2} / \mathrm{s}$ & $3.96 \mathrm{E}-06$ & $3.10 \mathrm{E}-06$ & 7.60E-06 & $9.04 \mathrm{E}-06$ \\
\hline $\begin{array}{ll}\text { Injected } & \mathrm{mg} \\
\text { Concentration } & \mathrm{DP}\end{array}$ & & 5080 & 3010 & 407706 & 320508 \\
\hline \multicolumn{6}{|c|}{ Fit Parameters } \\
\hline Sum of Residuals, SR & -- & $1.55 \mathrm{E}-01$ & $1.83 \mathrm{E}-01$ & $1.01 \mathrm{E}-01$ & $1.06 \mathrm{E}-01$ \\
\hline Peclet number, $N_{P e}$ & -- & 115 & 115 & 115 & 115 \\
\hline Matrix number, $N_{M a}$ & -- & 12.8 & 10.0 & 24.5 & 29.1 \\
\hline Matrix porosity, $\phi_{m}$ & -- & 0.18 & 0.18 & 0.18 & 0.18 \\
\hline \multicolumn{6}{|c|}{ Parameters Calculated from Fit and Known Parameters } \\
\hline Velocity, $u$ & $\mathrm{~cm} / \mathrm{s}$ & 0.023 & & & \\
\hline Fracture Width, $2 b$ & $\mathrm{~cm}$ & 0.024 & & & \\
\hline Disp. Coeff., $D_{L}$ & $\mathrm{~cm}^{2} / \mathrm{s}$ & 0.009 & & & \\
\hline Fracture Volume & $\mathrm{mL}$ & 5.5 & & & \\
\hline Fracture MRT & $\mathrm{s}$ & 1956 & & & \\
\hline
\end{tabular}


Table 5.6: Experiment Characteristics and Results for Frac8

\begin{tabular}{|c|c|c|c|}
\hline \multicolumn{4}{|c|}{ Known or Measured Parameters } \\
\hline \multicolumn{2}{|l|}{ Rock Type } & \multicolumn{2}{|c|}{ Berea } \\
\hline Column Length, $x_{L}$ & $\mathrm{~cm}$ & \multicolumn{2}{|l|}{45.9} \\
\hline Column Width, $w$ & $\mathrm{~cm}$ & \multicolumn{2}{|l|}{4.9} \\
\hline Flow Rate, $q$ & $\mathrm{~mL} / \mathrm{s}$ & \multicolumn{2}{|l|}{0.018} \\
\hline Slug Size, $t_{s}$ & $\mathrm{~mL}$ & \multicolumn{2}{|l|}{3.27} \\
\hline \multirow{3}{*}{$\begin{array}{l}\text { Tracer } \\
\text { Eff. Diff. Coeff, } D_{m} \\
\text { Injected Conc. }\end{array}$} & & IPA & Hexanol \\
\hline & $\mathrm{cm}^{2} / \mathrm{s}$ & $3.96 \mathrm{E}-06$ & $3.10 \mathrm{E}-06$ \\
\hline & $\mathrm{mg} / \mathrm{L}$ & 5080 & 3010 \\
\hline \multicolumn{4}{|c|}{ Fit Parameters } \\
\hline \multirow{2}{*}{$\begin{array}{l}\text { Sum of Residuals, SR } \\
\text { Peclet number, } N_{P e}\end{array}$} & -- & \multirow{2}{*}{$\begin{array}{l}1.46 \mathrm{E}-01 \\
13\end{array}$} & \multirow{2}{*}{$\begin{array}{l}1.63 \mathrm{E}-01 \\
13\end{array}$} \\
\hline & -- & & \\
\hline Matrix number, $N_{M a}$ & -- & 1.4 & 1.1 \\
\hline Matrix porosity, $\phi_{m}$ & -- & 0.18 & 0.18 \\
\hline \multicolumn{4}{|c|}{ Parameters Calculated from Fit and Known Parameters } \\
\hline \multicolumn{4}{|c|}{ Velocity, $u \quad \mathrm{~cm} / \mathrm{s} \quad 0.102$} \\
\hline Fracture Width, $2 b$ & $\mathrm{~cm}$ & \multicolumn{2}{|l|}{0.0361} \\
\hline Disp. Coeff., $D_{L}$ & $\mathrm{~cm}^{2}$ & \multicolumn{2}{|l|}{0.360} \\
\hline Fracture Volume & $\mathrm{mL}$ & \multicolumn{2}{|l|}{8.1} \\
\hline Fracture MRT & $\mathrm{s}$ & \multicolumn{2}{|l|}{451} \\
\hline Dispersivity, $\alpha$ & $\mathrm{s}^{1.5} / \mathrm{c}$ & \multicolumn{2}{|l|}{100} \\
\hline Dispersivity Exponent & $\beta---$ & \multicolumn{2}{|l|}{2.5} \\
\hline
\end{tabular}

Table 5.7: Partition Coefficient Measurements

\begin{tabular}{|l|c|c|}
\cline { 2 - 3 } \multicolumn{1}{c|}{} & \multicolumn{2}{c|}{ K $(\mathrm{mg} / \mathrm{L}) /(\mathrm{mg} / \mathrm{L})$} \\
\hline Vial & Hexanol & Heptanol \\
\hline 1 & 5.91 & 23.3 \\
\hline 2 & 5.44 & 21.8 \\
\hline 3 & 6.00 & 22.4 \\
\hline 4 & 5.41 & 23.4 \\
\hline 5 & 5.19 & 21.8 \\
\hline 6 & 5.65 & 21.7 \\
\hline uniformity coefficient & 0.051 & 0.031 \\
\hline average \pm 1 std. & $5.6 \pm 0.3$ & $22.4 \pm 0.7$ \\
\hline
\end{tabular}


Table 5.8: Experiment Characteristics and Results for Frac11

\begin{tabular}{|c|c|c|c|}
\hline \multicolumn{4}{|c|}{ Known or Measured Parameters } \\
\hline Rock Type & Berea & & \\
\hline Column Length, $x_{L} \quad \mathrm{~cm}$ & 60 & & \\
\hline Column Width, $w$ & 4.9 & & \\
\hline Flow Rate, $q$ & $3.3 \mathrm{E}-04$ & & \\
\hline Slug Size, $t_{s}$ & 10.0 & & \\
\hline Decane Volume & $1.8 \pm 0.4$ & & \\
\hline Tracer & IPA & Hexanol & Heptanol \\
\hline Eff. Diff. Coeff, $D_{m} \quad \mathrm{~cm}^{2} / \mathrm{s}$ & $3.96 \mathrm{E}-06$ & $3.10 \mathrm{E}-06$ & $2.80 \mathrm{E}-06$ \\
\hline Injected Conc. $\quad \mathrm{mg} / \mathrm{L}$ & 2026 & 2004 & 2006 \\
\hline \multicolumn{4}{|c|}{ Fit Parameters } \\
\hline Sum of Residuals, SR & $1.48 \mathrm{E}-02$ & $9.11 \mathrm{E}-03$ & $3.26 \mathrm{E}-02$ \\
\hline Peclet number, $N_{P e}$ & 130 & 130 & 130 \\
\hline Matrix number, $N_{M a}$ & 105.3 & 82.6 & 74.5 \\
\hline Matrix porosity, $\phi_{m}$ & 0.19 & 0.19 & 0.19 \\
\hline Decane Saturation & 0.089 & 0.089 & 0.089 \\
\hline \multicolumn{4}{|c|}{ Parameters Calculated from Fit and Known Parameters } \\
\hline Velocity, $u \quad \mathrm{~cm} / \mathrm{s}$ & 0.0021 & & \\
\hline Fracture Width, $2 b$ & 0.033 & & \\
\hline Disp. Coeff., $D_{L}$ & $9.47 \mathrm{E}-04$ & & \\
\hline Fracture Volume & 9.7 & & \\
\hline Fracture MRT & 29246 & & \\
\hline Decane Volume & 0.95 & & \\
\hline
\end{tabular}

Table 5.9: Sensitivity of Hexanol MRV and NAPL Volume Prediction to Data Extrapolation

\begin{tabular}{|l|l|l|l|l|l|l|}
\hline $\begin{array}{l}\text { Extrapolation } \\
\text { Start }\end{array}$ & slope & $\begin{array}{l}\% \\
\text { change }\end{array}$ & $\begin{array}{l}\text { MRV } \\
(\mathrm{mL})\end{array}$ & $\begin{array}{l}\% \\
\text { change }\end{array}$ & $\begin{array}{l}V_{N} \\
(\mathrm{~mL})\end{array}$ & $\begin{array}{l}\% \\
\text { change }\end{array}$ \\
\hline $63 \mathrm{~mL}$ & -0.015 & & 94 & & 0.88 & \\
\hline $63 \mathrm{~mL}$ & -0.013 & 13 & 104 & 11 & 2.66 & 204 \\
\hline $63 \mathrm{~mL}$ & -0.017 & -13 & 87 & -7 & -0.37 & -143 \\
\hline $174 \mathrm{~mL}$ & -0.009 & & 120 & & 5.52 & \\
\hline $174 \mathrm{~mL}$ & -0.007 & 22 & 137 & -14 & 8.55 & 55 \\
\hline $174 \mathrm{~mL}$ & -0.011 & -22 & 110 & 8 & 3.73 & -32 \\
\hline
\end{tabular}


Table 5.10: Experiment Characteristics and Results for Frac12

\begin{tabular}{|c|c|c|c|}
\hline \multicolumn{4}{|c|}{ Known or Measured Parameters } \\
\hline Rock Type & \multicolumn{3}{|l|}{ Berea } \\
\hline Column Length, $x_{L} \quad \mathrm{~cm}$ & \multicolumn{3}{|l|}{60} \\
\hline Column Width, $w$ & \multicolumn{3}{|l|}{4.9} \\
\hline Flow Rate, $q$ & \multicolumn{3}{|l|}{0.00362} \\
\hline Slug Size, $t_{s}$ & \multicolumn{3}{|l|}{10} \\
\hline Decane Volume & \multicolumn{3}{|l|}{$1.8 \pm 0.4$} \\
\hline Tracer & IPA & Hexanol & Heptanol \\
\hline Eff. Diff. Coeff, $D_{m} \quad \mathrm{~cm}^{2} / \mathrm{s}$ & $3.96 \mathrm{E}-06$ & $3.10 \mathrm{E}-06$ & $2.80 \mathrm{E}-06$ \\
\hline Injected Conc. $\quad \mathrm{mg} / \mathrm{L}$ & 2026 & 2004 & 2006 \\
\hline \multicolumn{4}{|l|}{ Fit Parameters } \\
\hline Sum of Residuals, SR & $1.86 \mathrm{E}-02$ & $2.10 \mathrm{E}-02$ & $5.07 \mathrm{E}-02$ \\
\hline Peclet number, $N_{P e}$ & 15 & 15 & 15 \\
\hline Matrix number, $N_{M a}$ & 8.9 & 7.0 & 6.3 \\
\hline \multirow{2}{*}{$\begin{array}{l}\text { Matrix porosity, } \phi_{m} \\
\text { Decane Saturation }\end{array}$} & 0.26 & 0.26 & 0.26 \\
\hline & 0.095 & 0.095 & 0.095 \\
\hline \multicolumn{4}{|c|}{ Parameters Calculated from Fit and Known Parameters } \\
\hline Velocity, $u$ & 0.0204 & & \\
\hline Fracture Width, $2 b$ & 0.036 & & \\
\hline Disp. Coeff., $D_{L}$ & $8.15 \mathrm{E}-02$ & & \\
\hline Fracture Volume & 10.7 & & \\
\hline Fracture MRT & 2945 & & \\
\hline Decane Volume & 1.1 & & \\
\hline
\end{tabular}




\subsection{Figures}
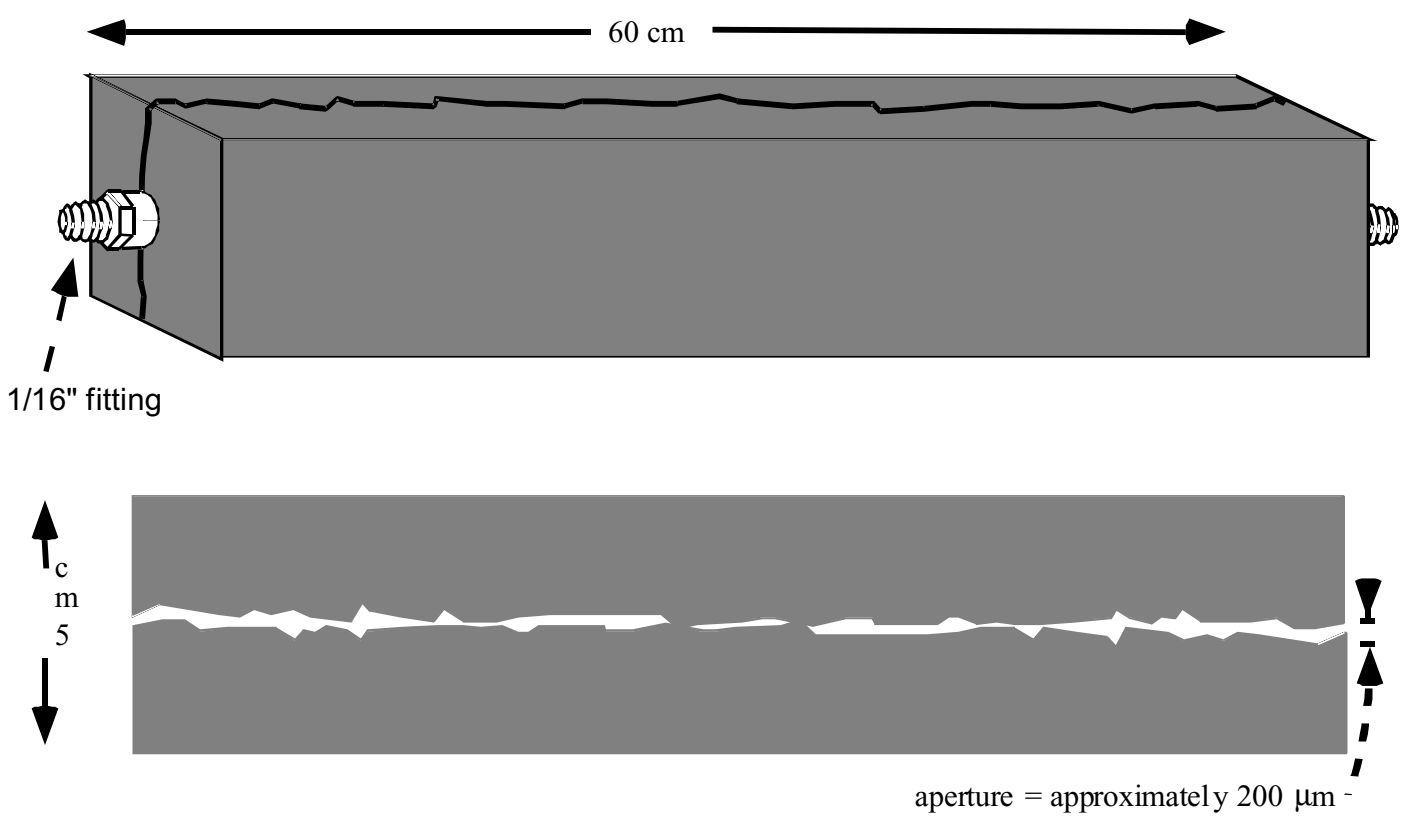

Figure 5.1: Illustration of prepared sample core.

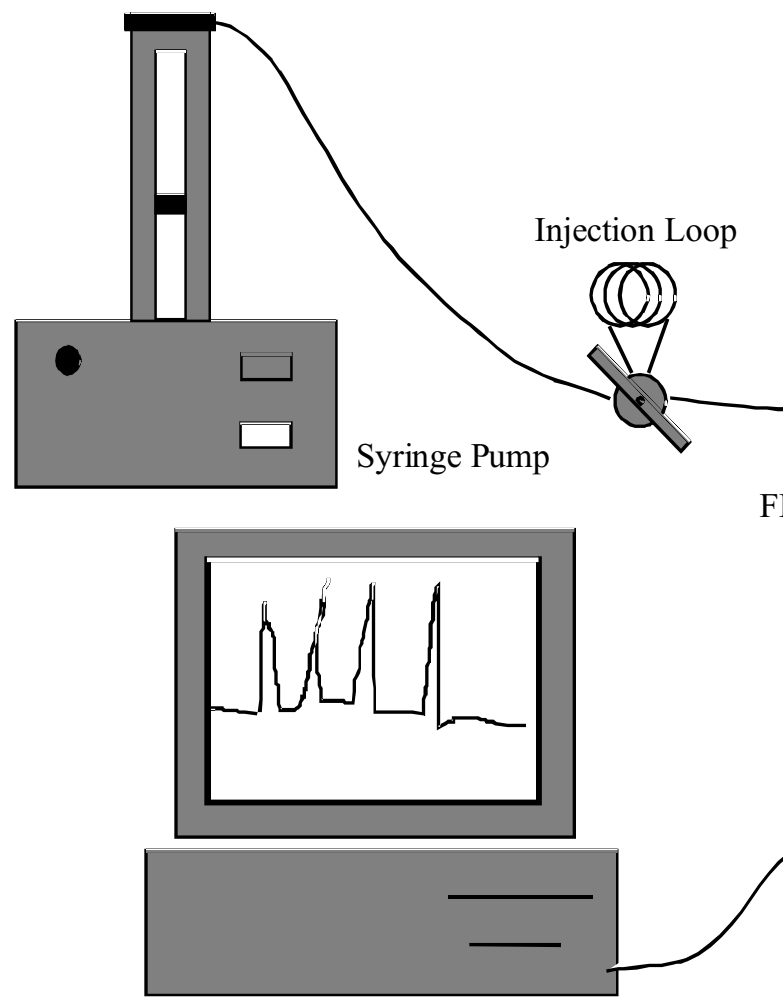

PC Control/Data Acquisition

Figure 5.2: Equipment setup for fracture experiments. 


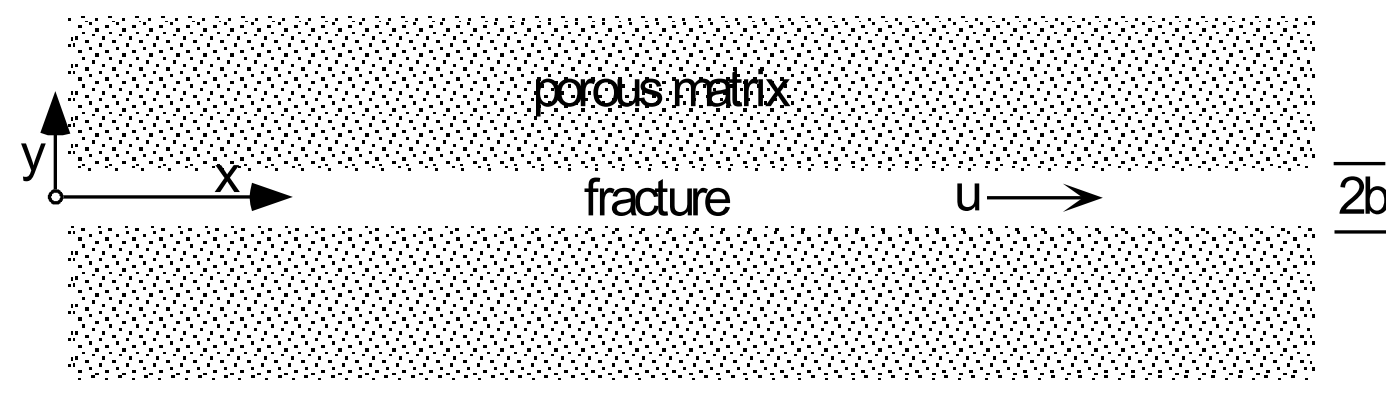

Figure 5.3: The fracture-matrix coordinate system for the Tang model.

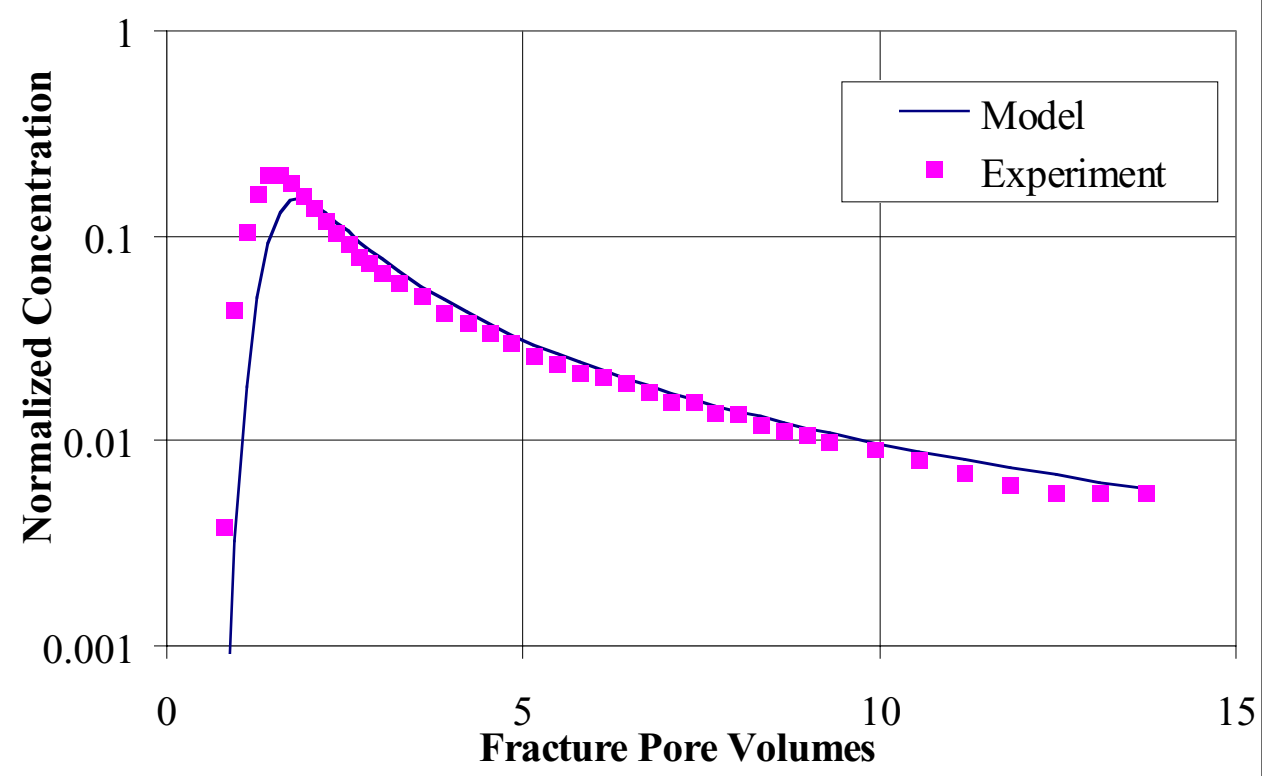

Figure 5.4: Frac5 comparison of model and experiment, $\phi_{m}=0.19$.

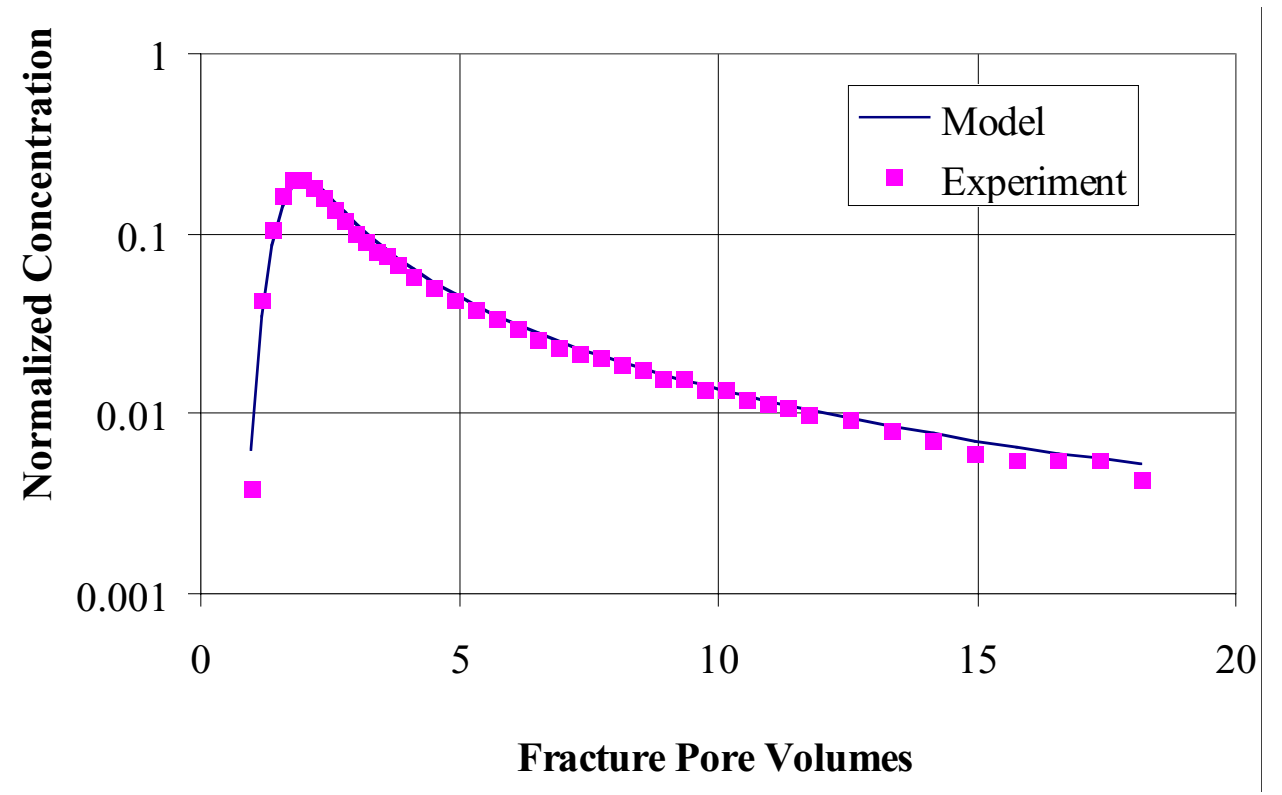

Figure 5.5: Frac5 comparison of model and experiment, $\phi_{m}=0.17$. 


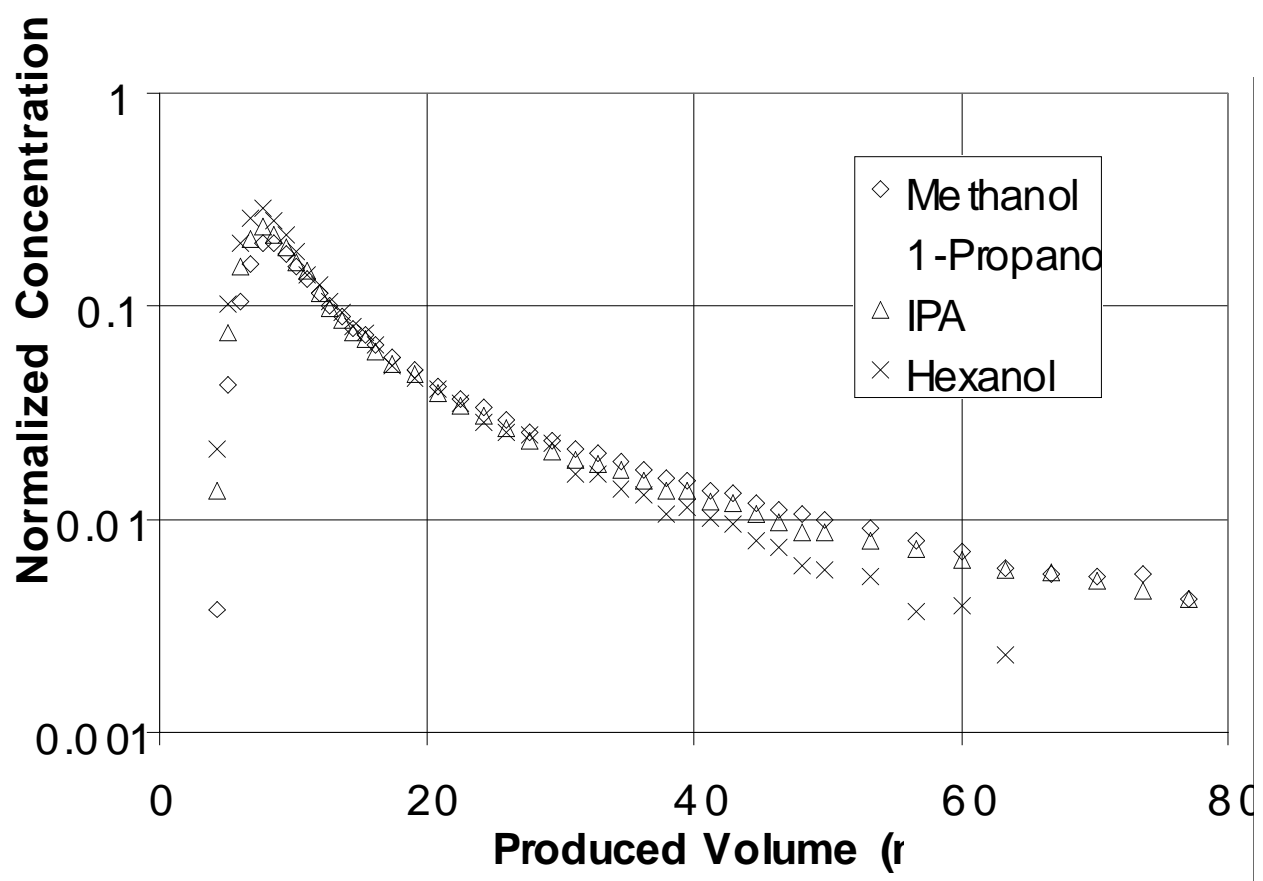

Figure 5.6: Tracer response data for experiment Frac5.

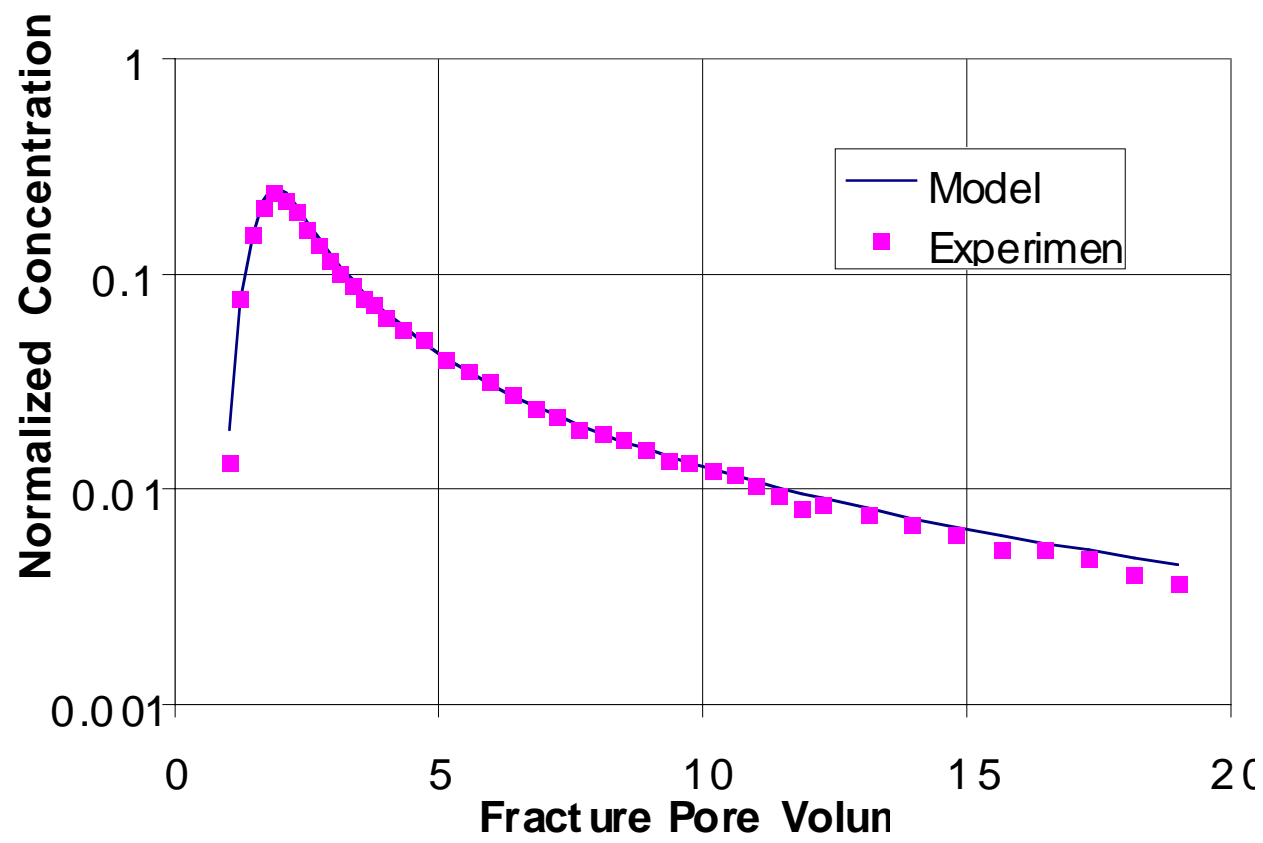

Figure 5.7: 1-propanol response data and fit model curve, Frac5. 

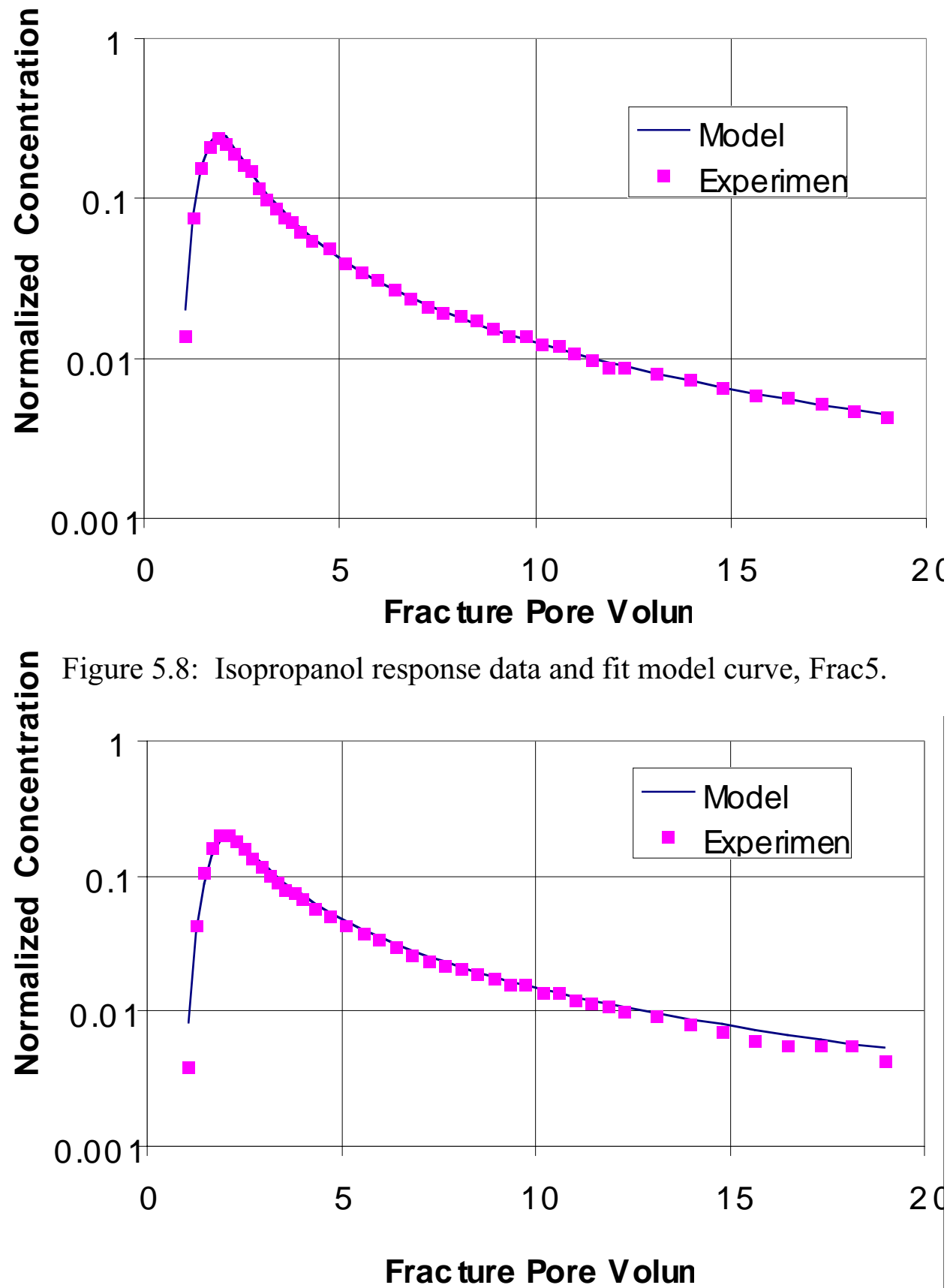

Figure 5.9: Methanol response data and fit model curve, Frac5. 


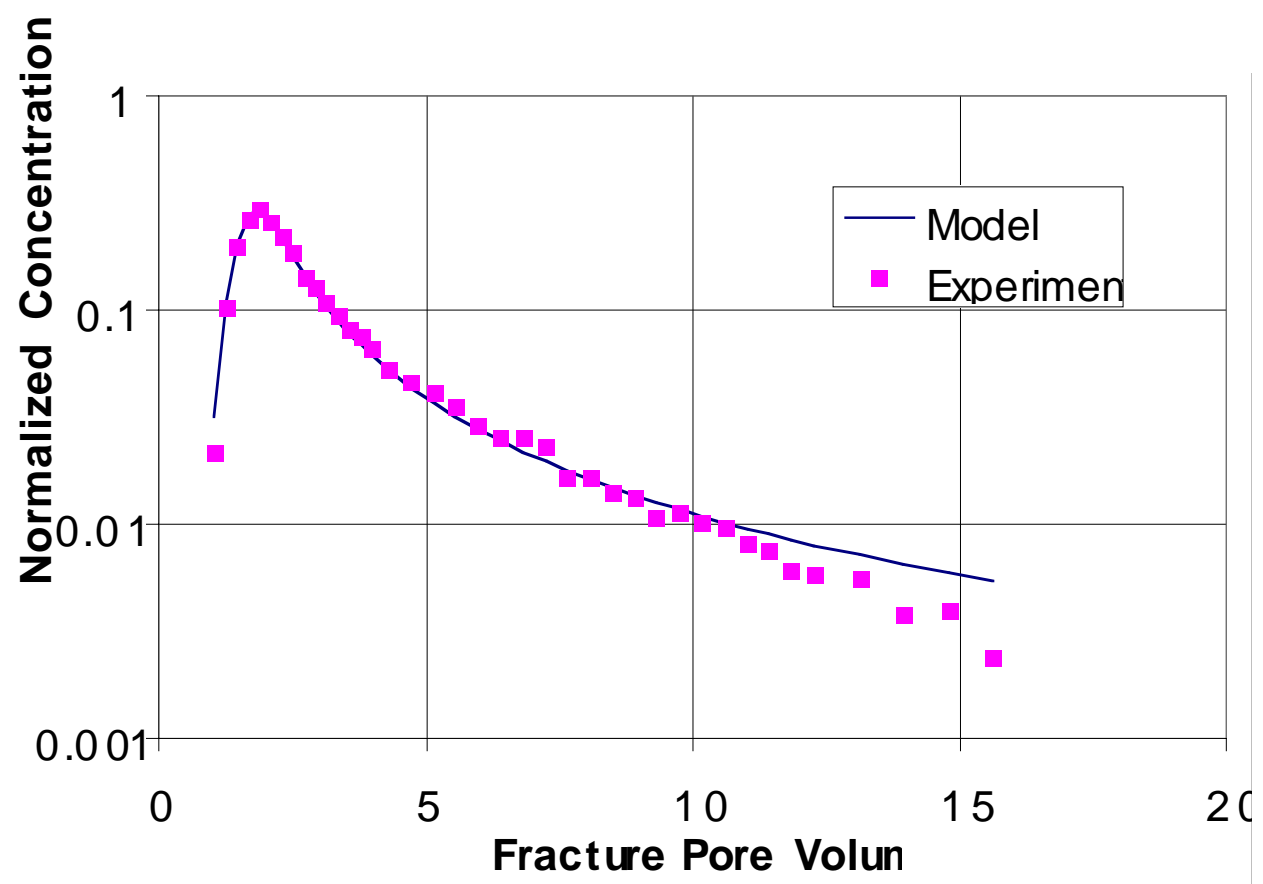

Figure 5.10: Hexanol response data and fit model curve, Frac5.

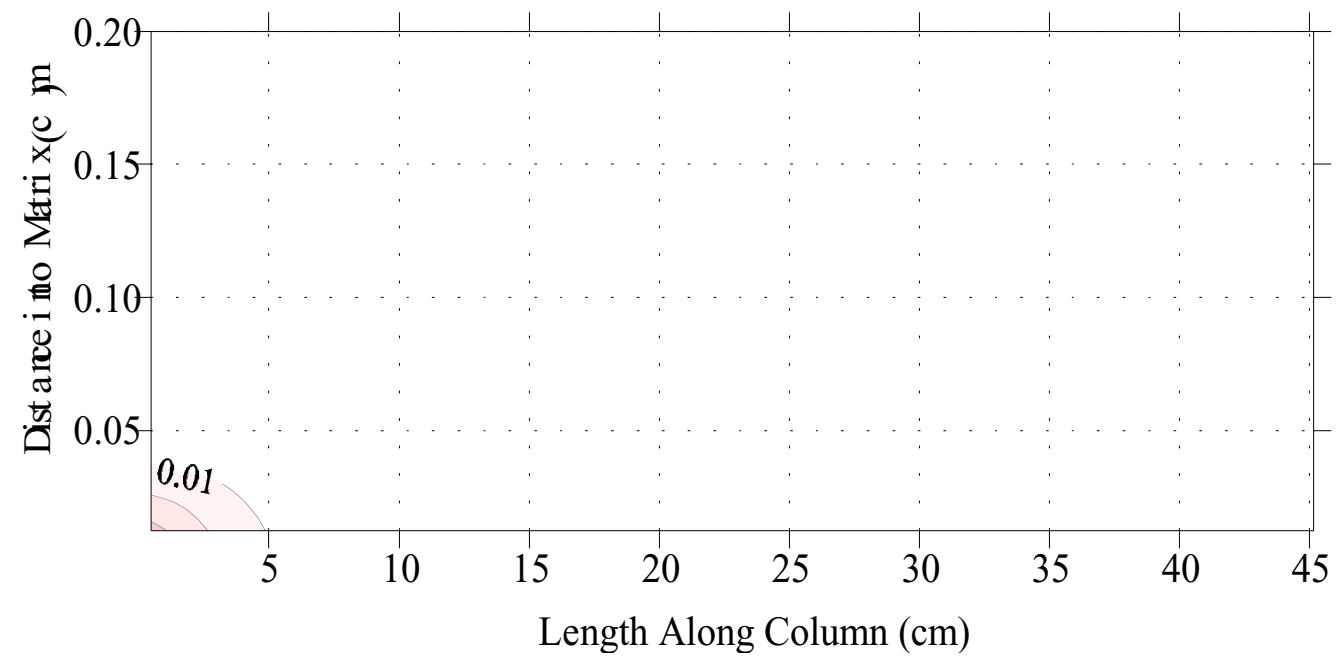

Figure 5.11: Normalized concentration in matrix at time $=3 \mathrm{~min}, 0.08 \mathrm{PV}$. 

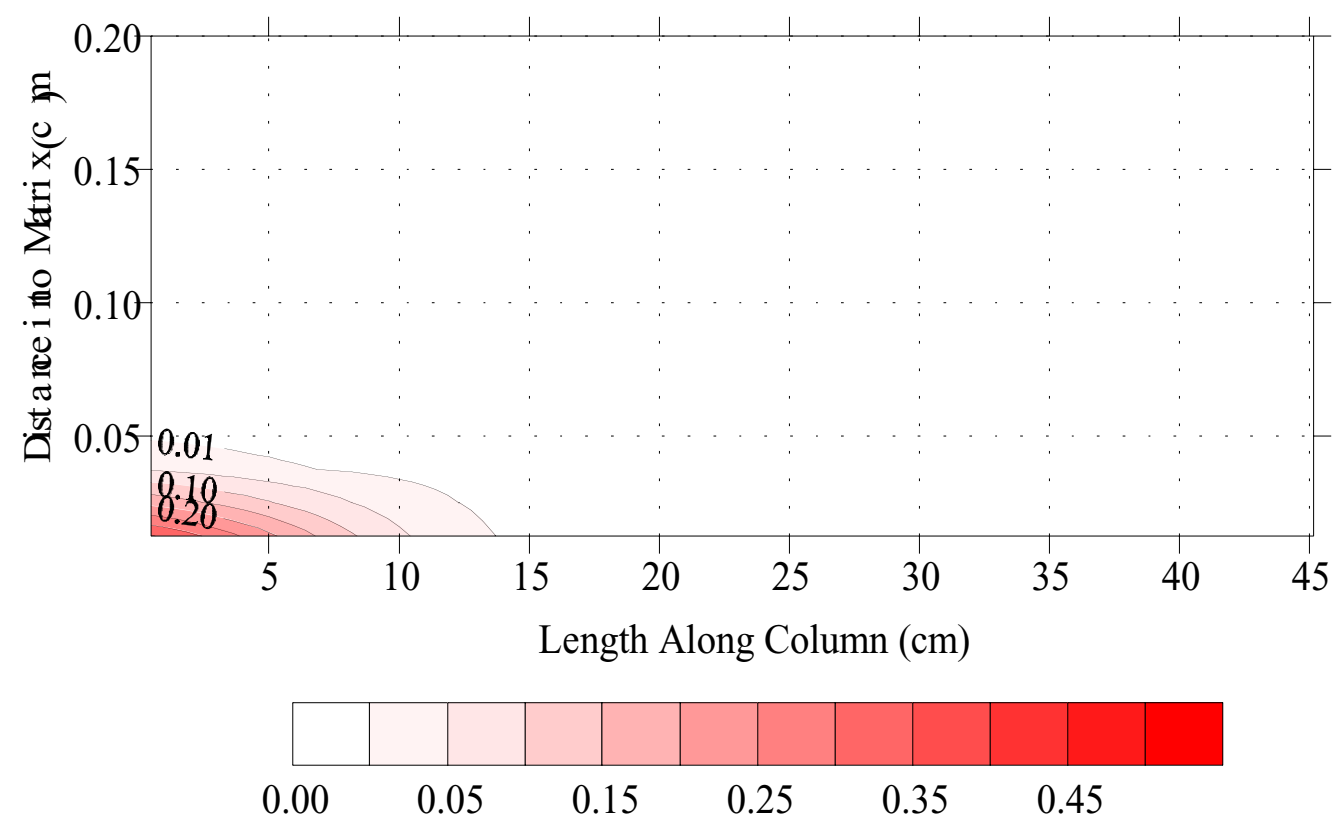

Figure 5.12: Normalized concentration in matrix at time $=9 \mathrm{~min}, 0.24 \mathrm{PV}$.

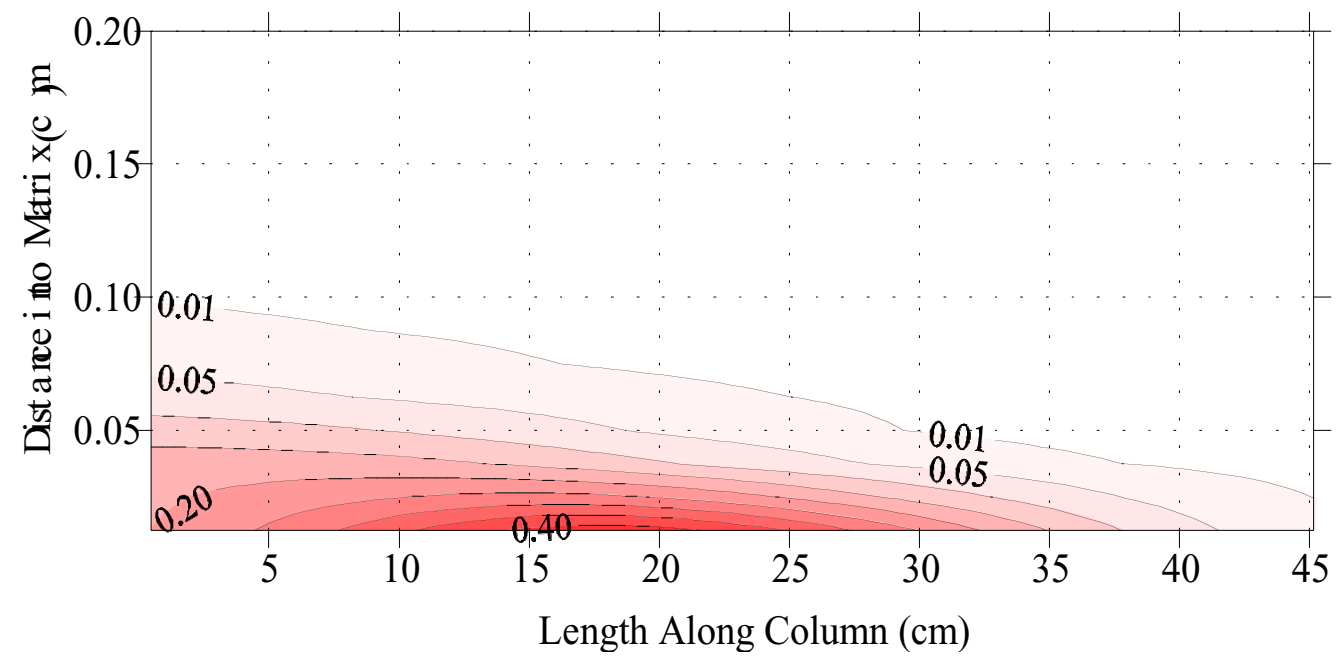

Figure 5.13: Normalized concentration in matrix at time $=33 \mathrm{~min}, .9 \mathrm{PV}$. 

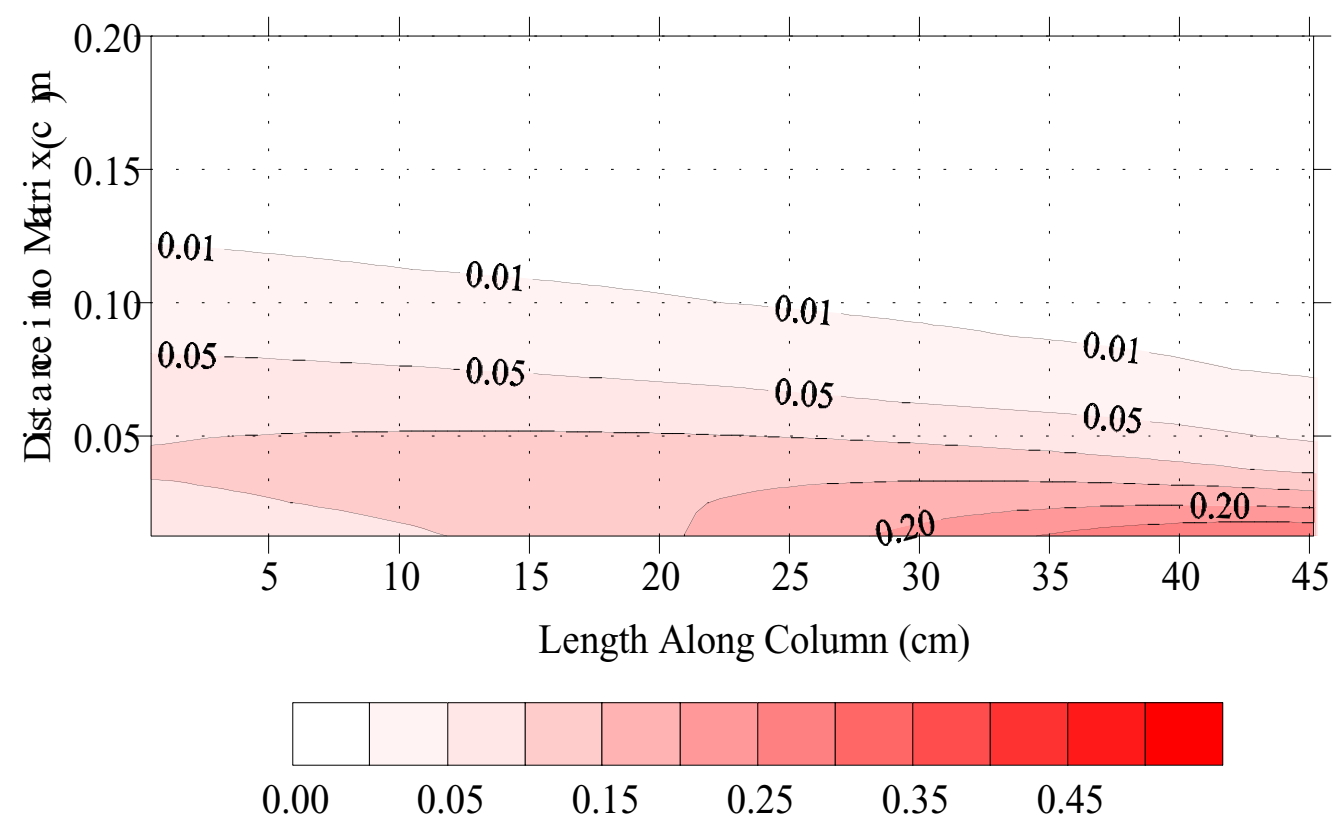

Figure 5.14: Normalized concentration in matrix at time $=56 \mathrm{~min}, 1.53 \mathrm{PV}$.

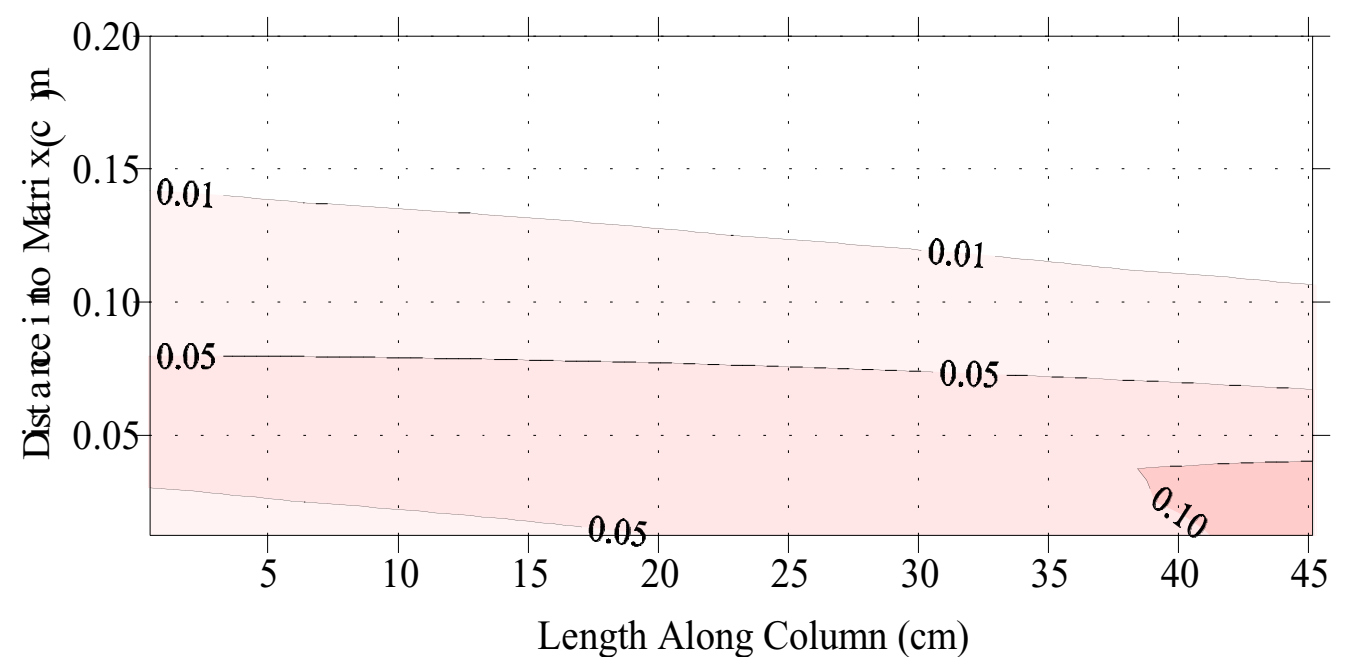

Figure 5.15: Normalized concentration in matrix at time $=85 \mathrm{~min}, 2.31 \mathrm{PV}$. 

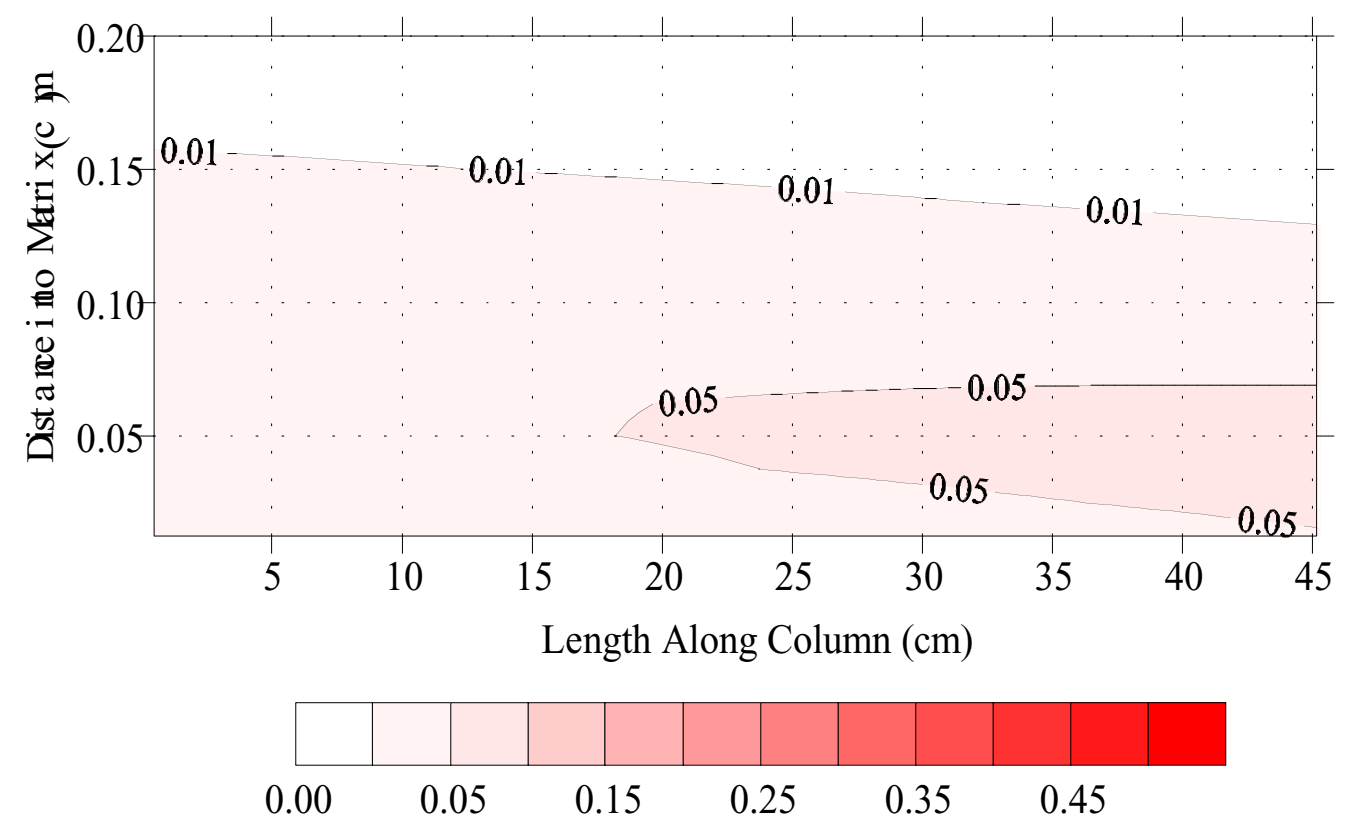

Figure 5.16: Normalized concentration in matrix at time $=117 \mathrm{~min}, 3.18 \mathrm{PV}$.

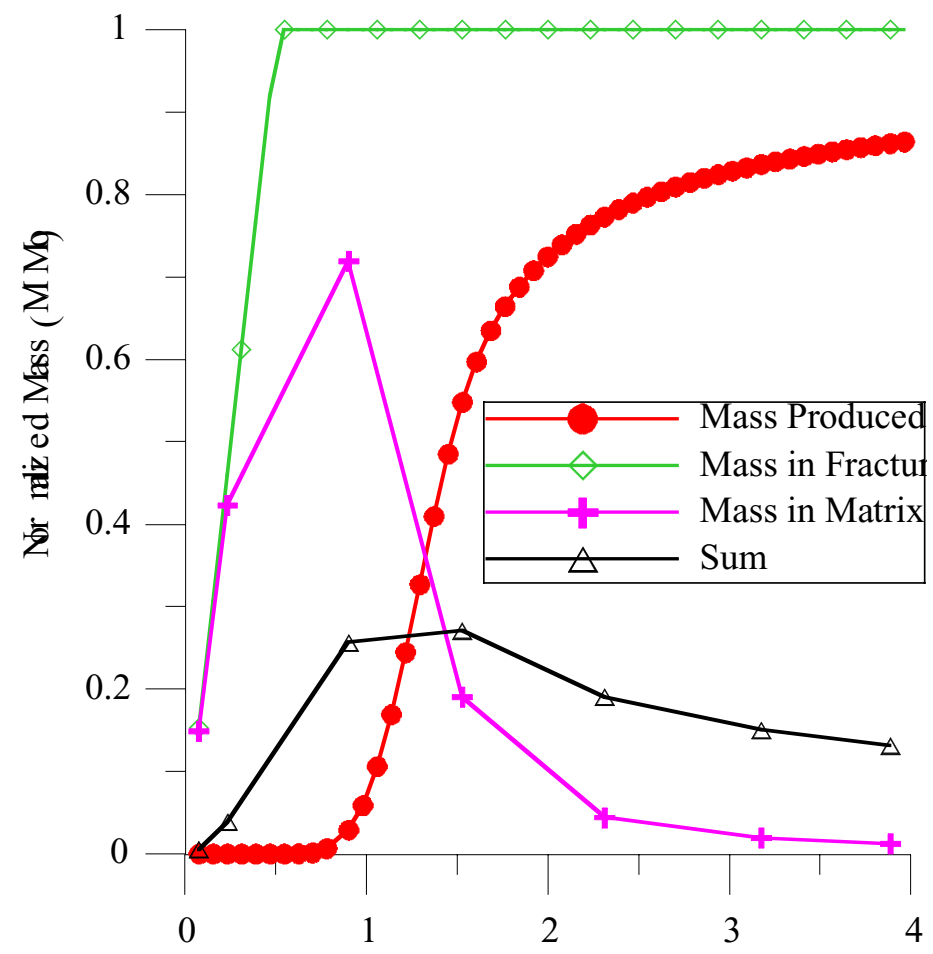

Pore Volumes

Figure 5.17: Mass history from UTCHEM simulation of Frac5. 


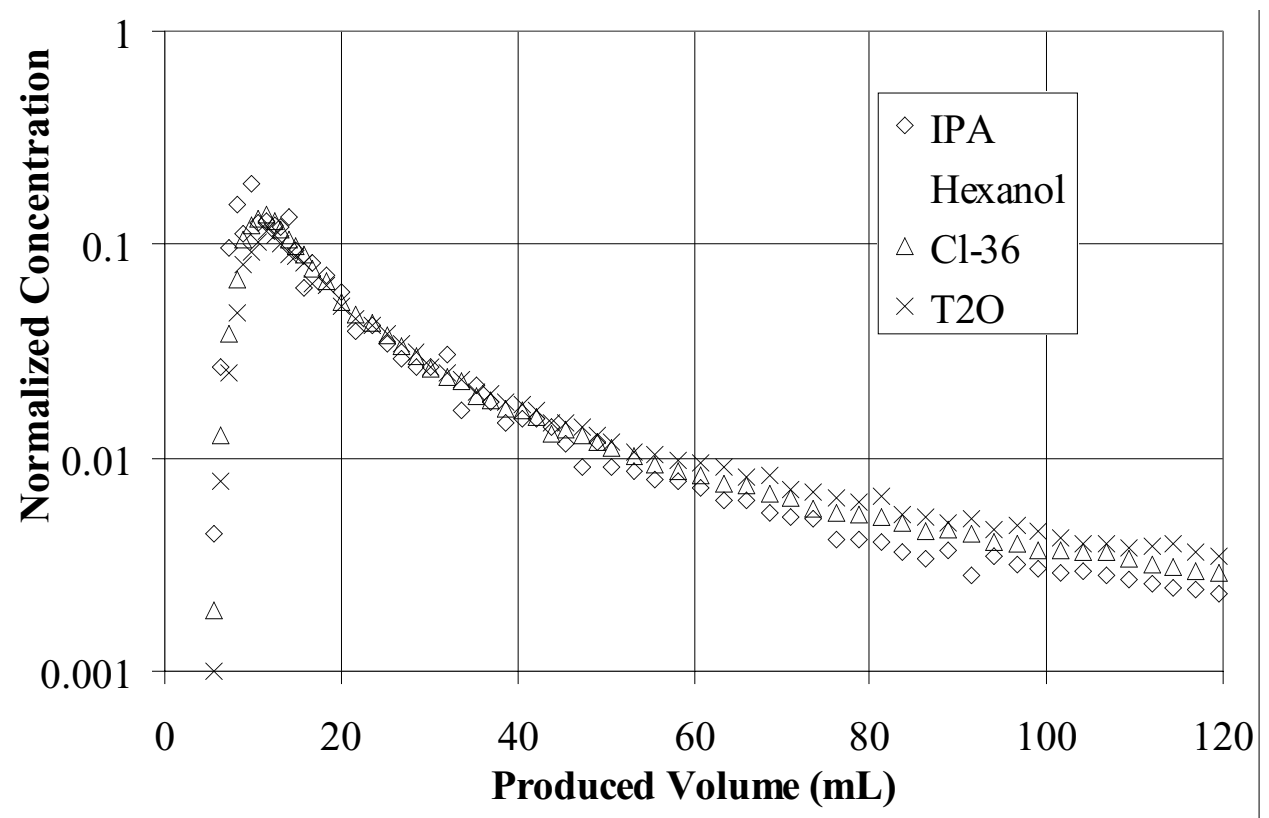

Figure 5.18: Tracer response data for experiment Frac6.

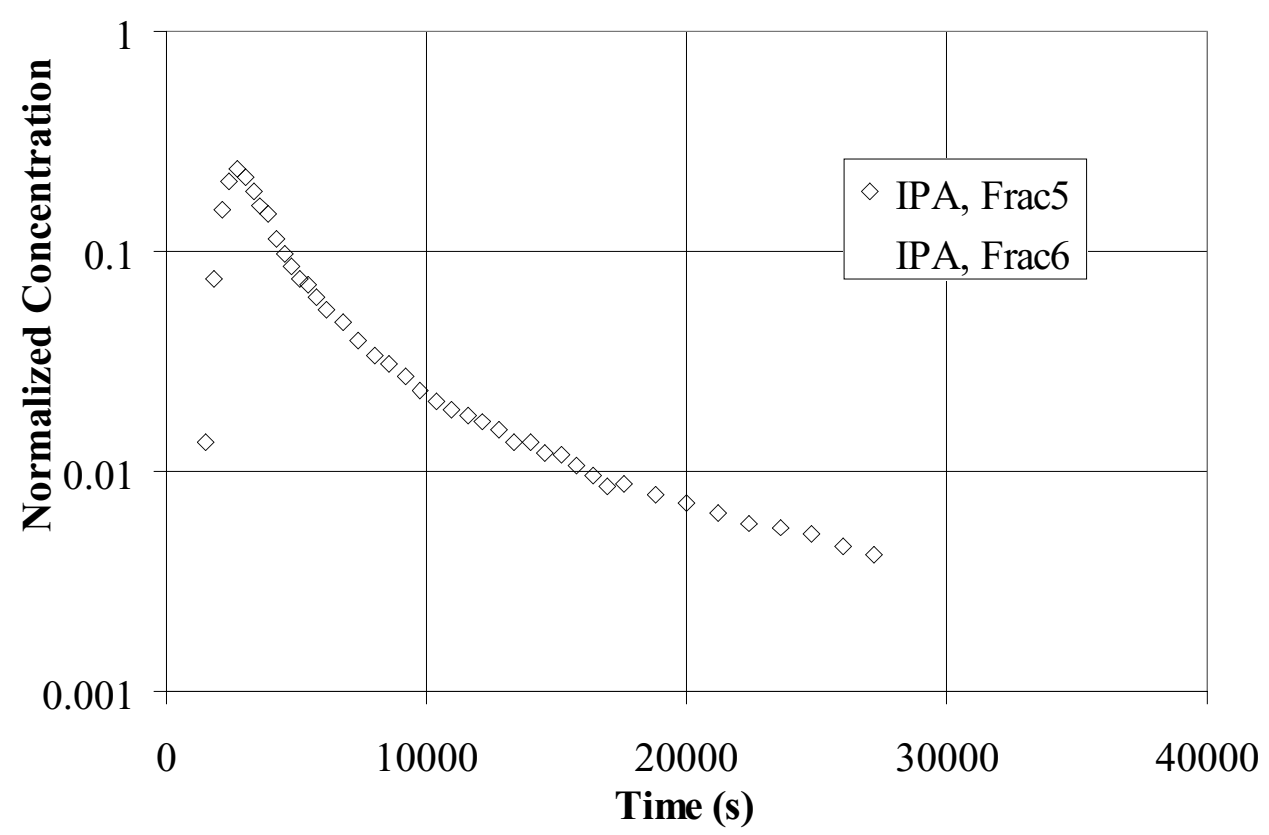

Figure 5.19: Comparison of IPA response data for Frac5 and Frac6. 


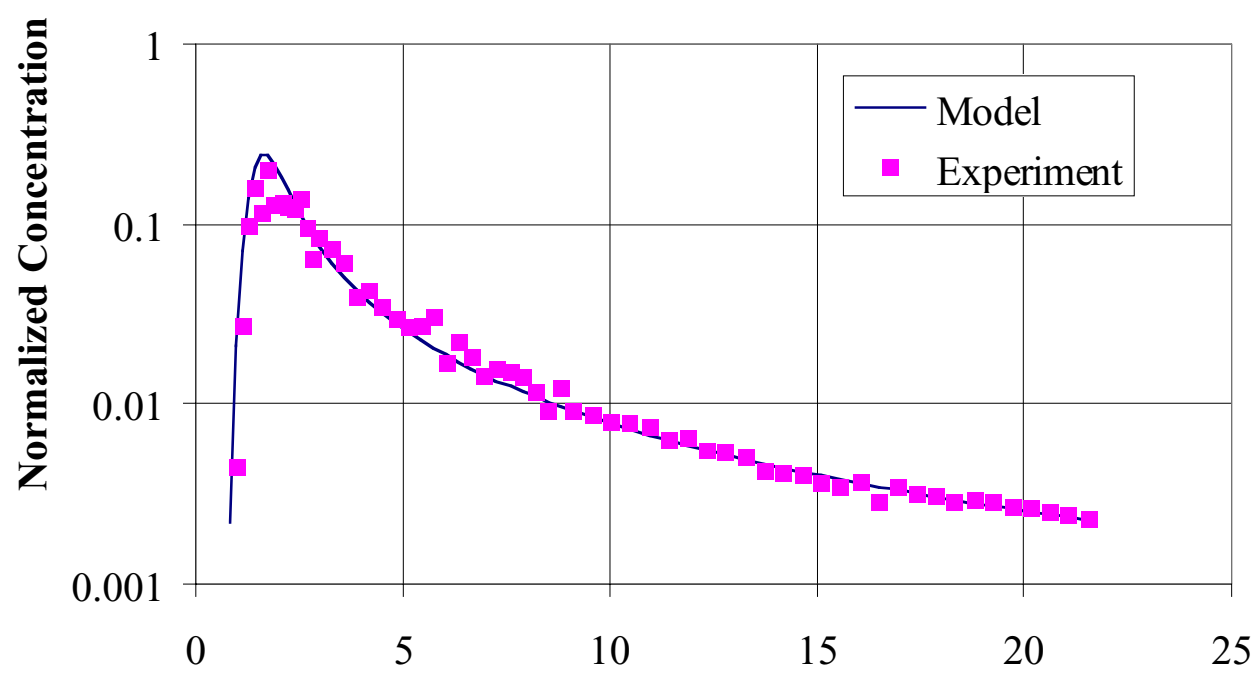

Fracture Pore Volumes

Figure 5.20: IPA response data and fit model curve, Frac6.

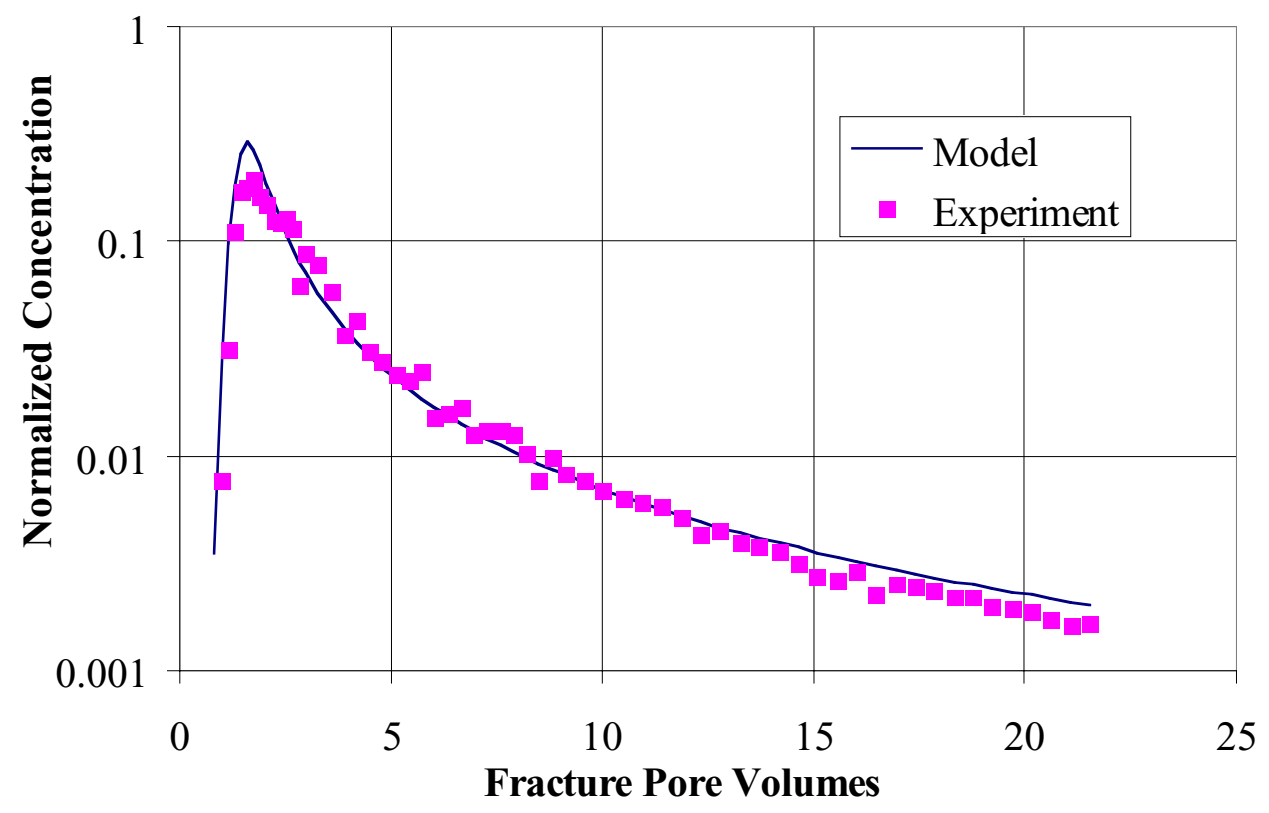

Figure 5.21: Hexanol response data and fit model curve, Frac6. 


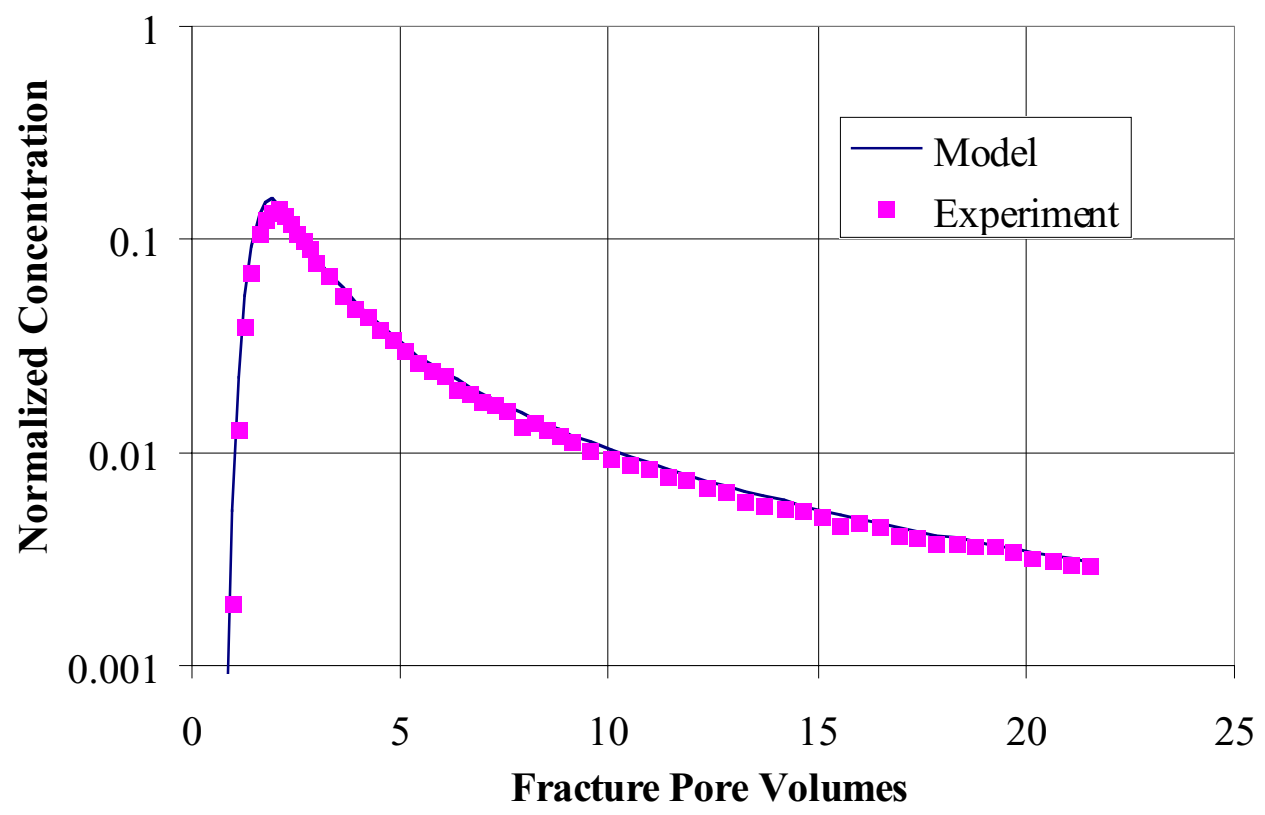

Figure 5.22: $\mathrm{Cl}^{36}$ response data and fit model curve, Frac6.

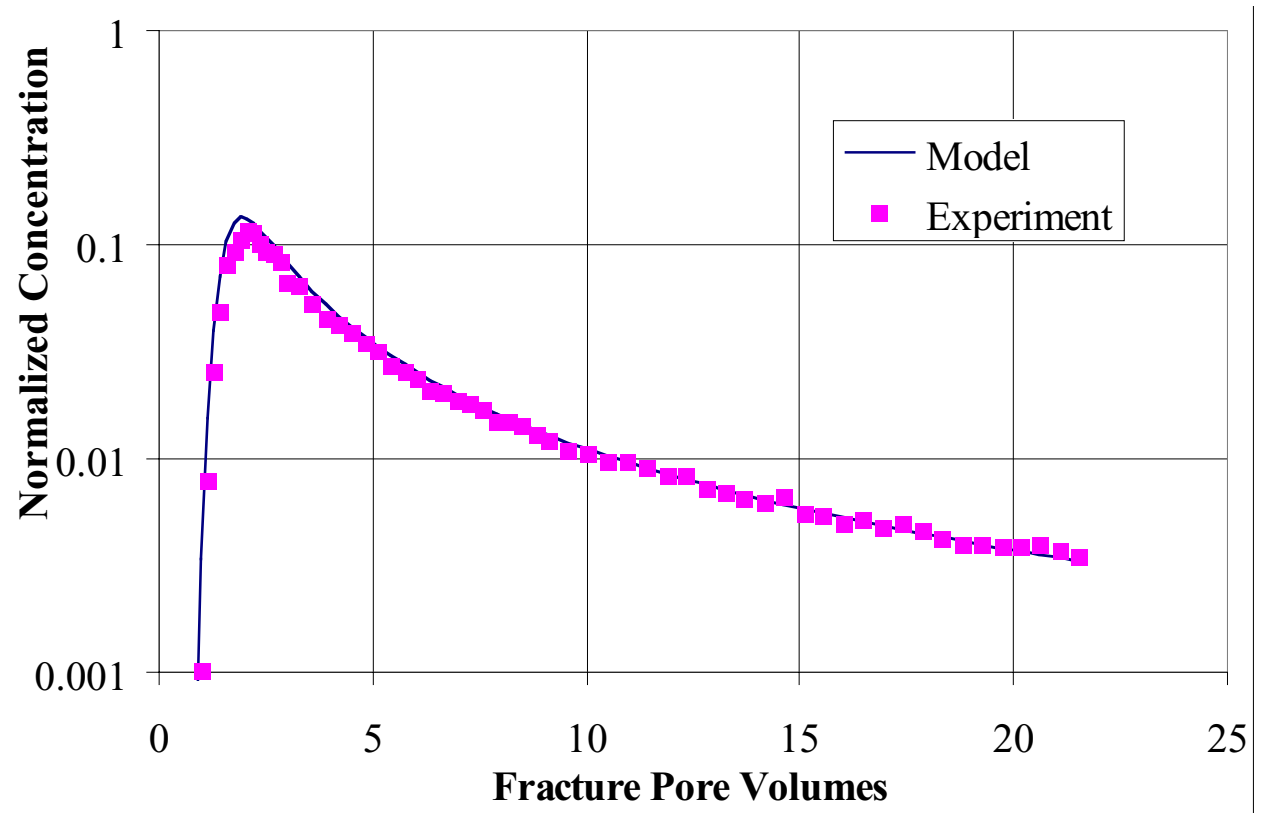

Figure 5.23: Tritiated water response data and fit model curve, Frac6. 


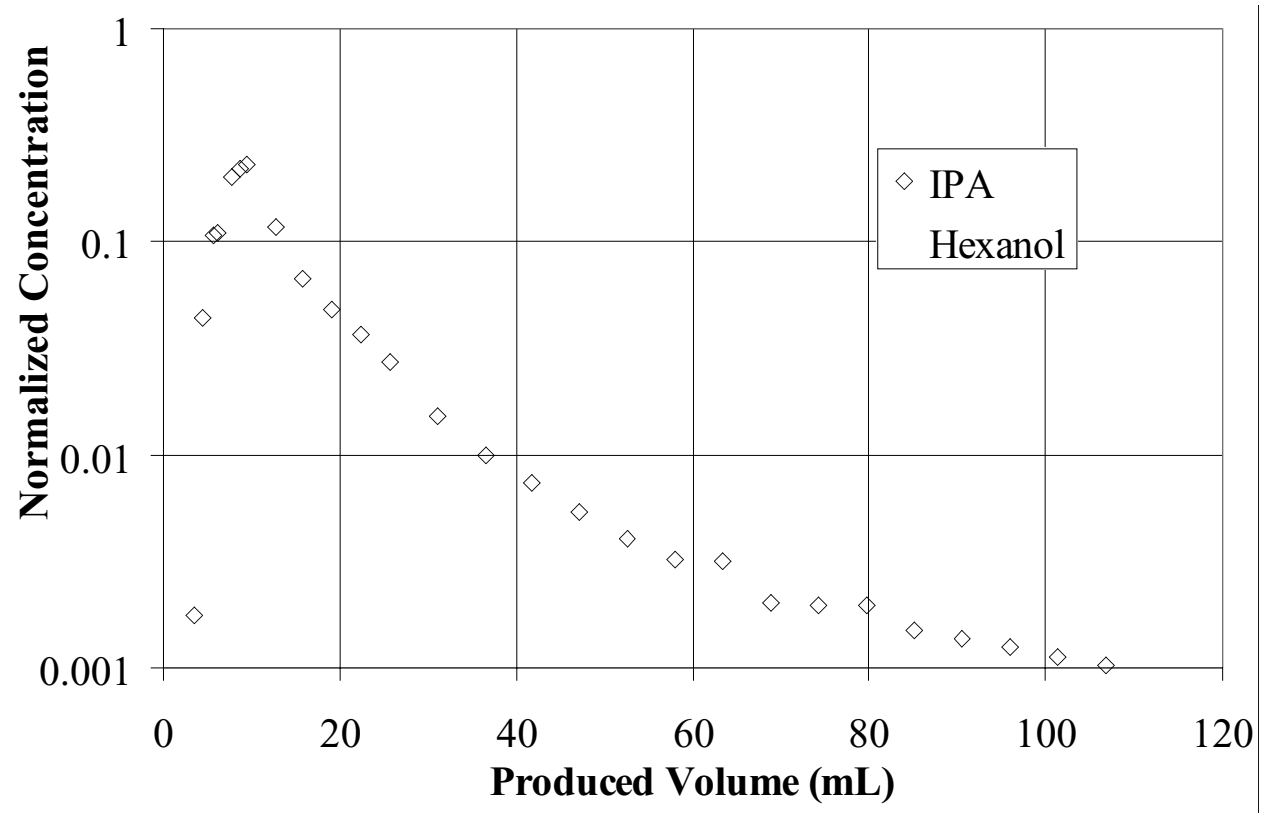

Figure 5.24: Tracer response data for experiment Frac8.

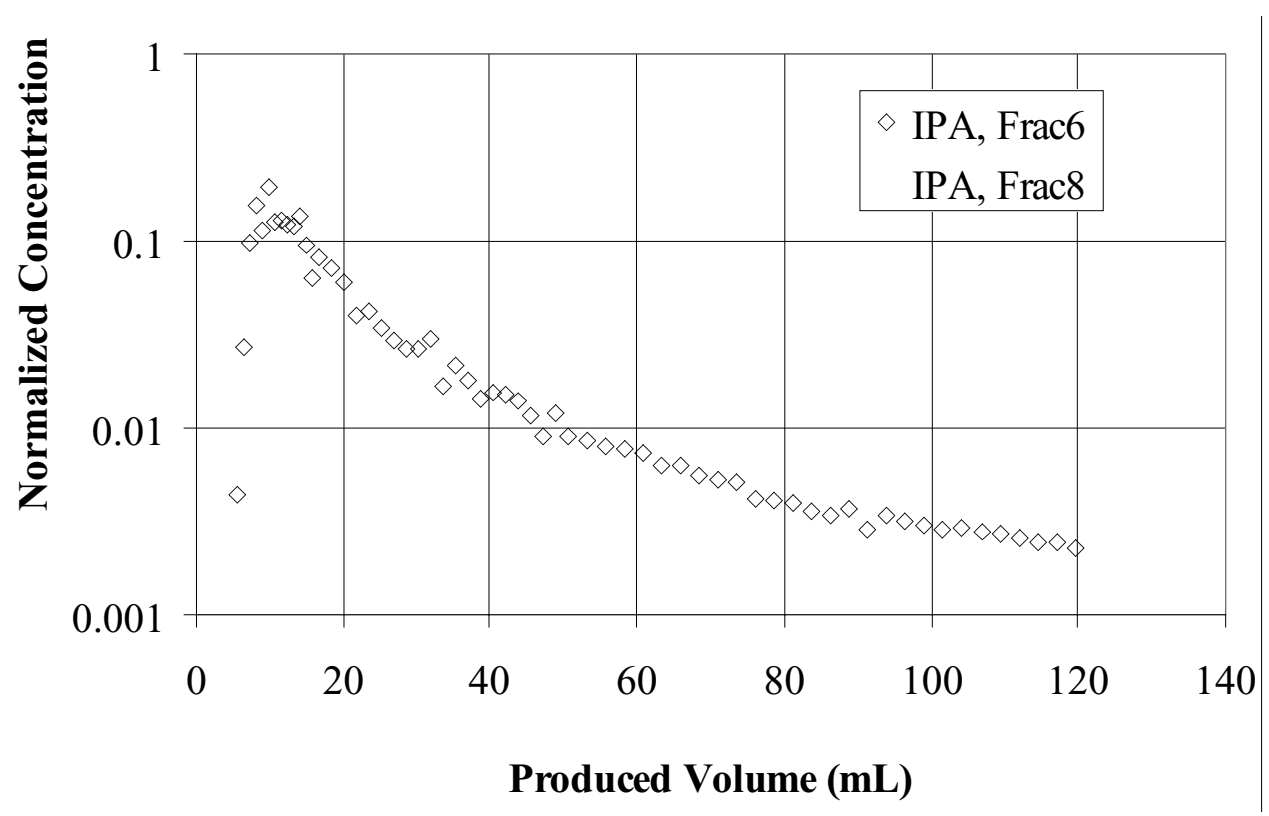

Figure 5.25: Comparison of IPA response data for Frac6 and Frac8. 


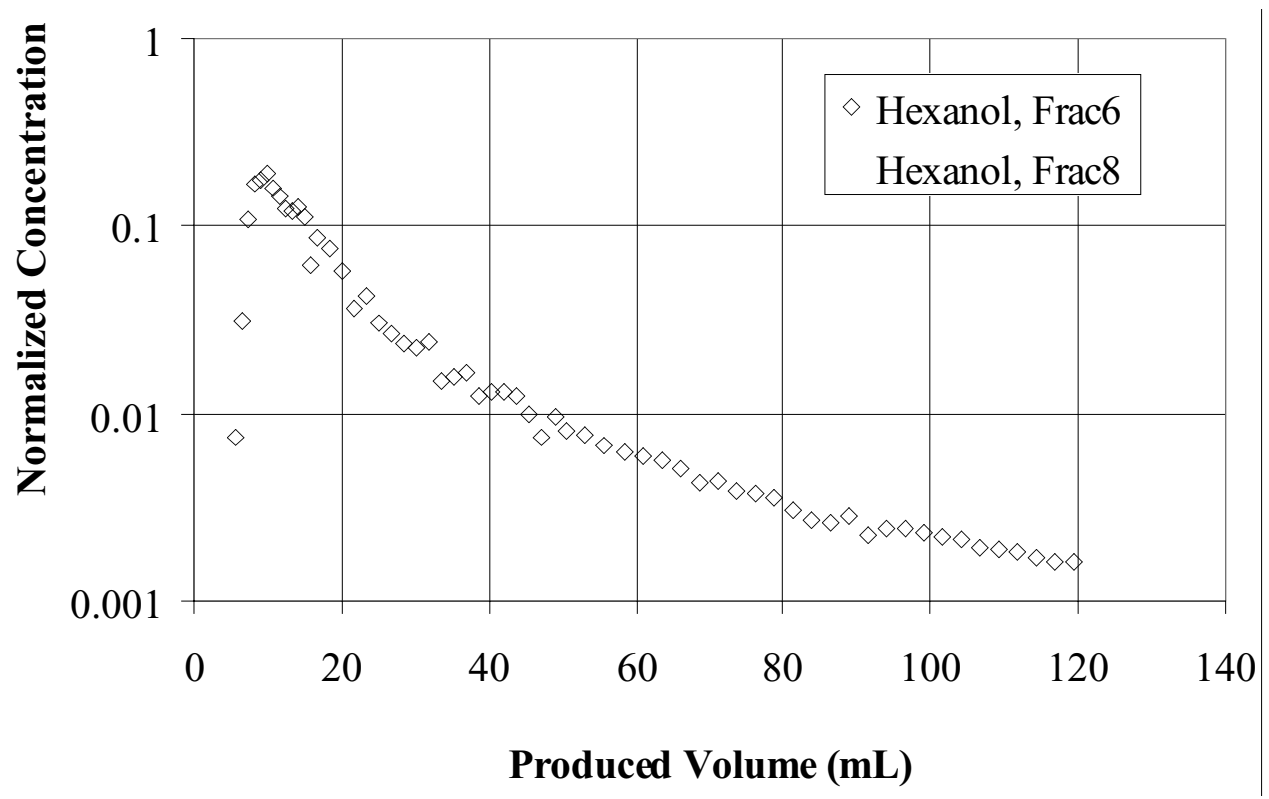

Figure 5.26: Comparison of hexanol response data for Frac6 and Frac8.

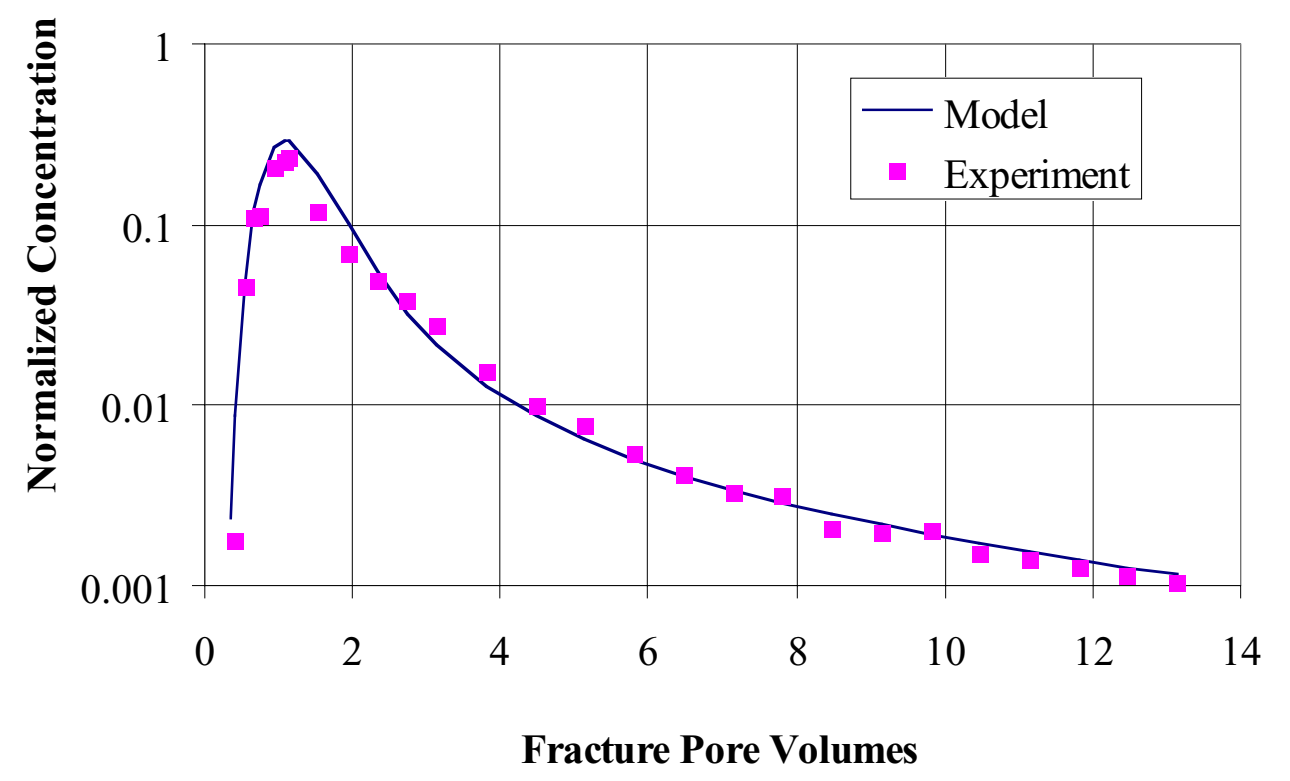

Figure 5.27: IPA response data and fit model curve, Frac8. 


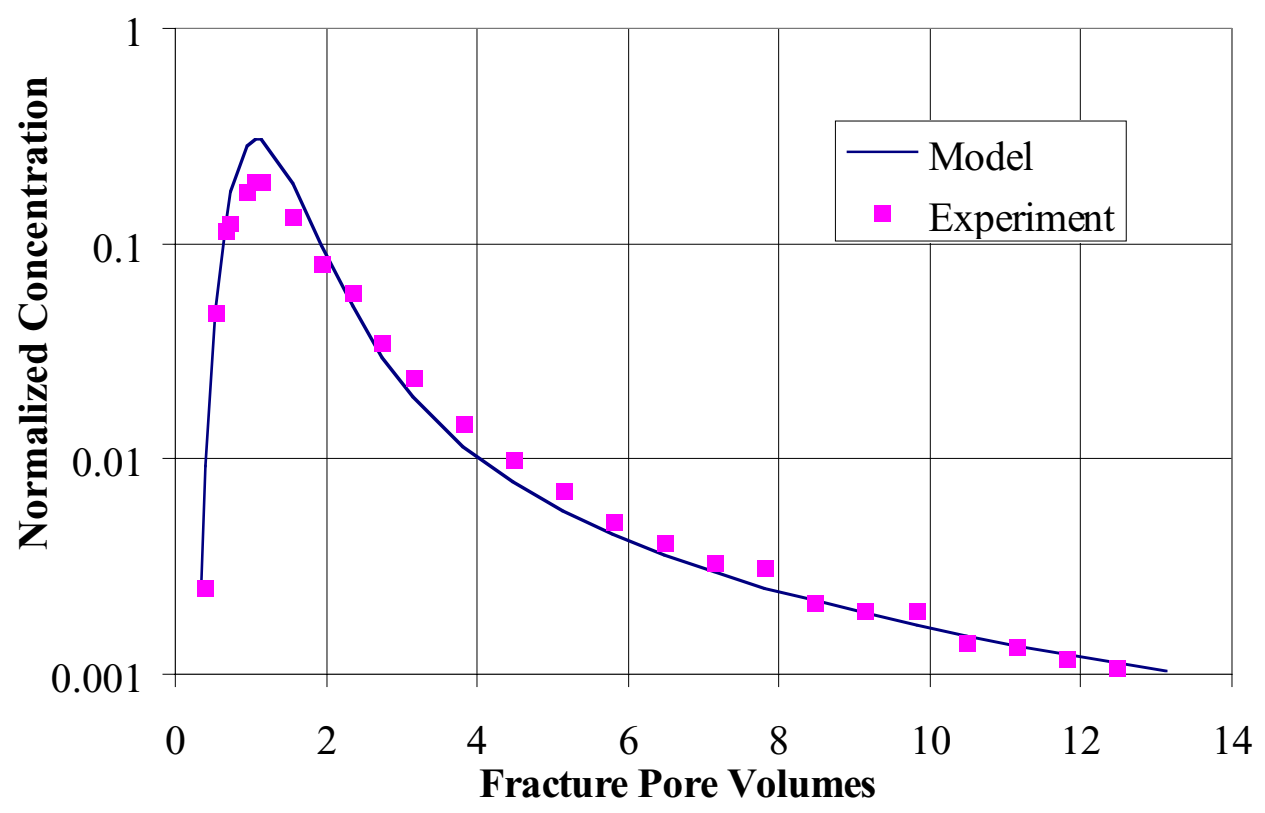

Figure 5.28: Hexanol response data and fit model curve, Frac8.

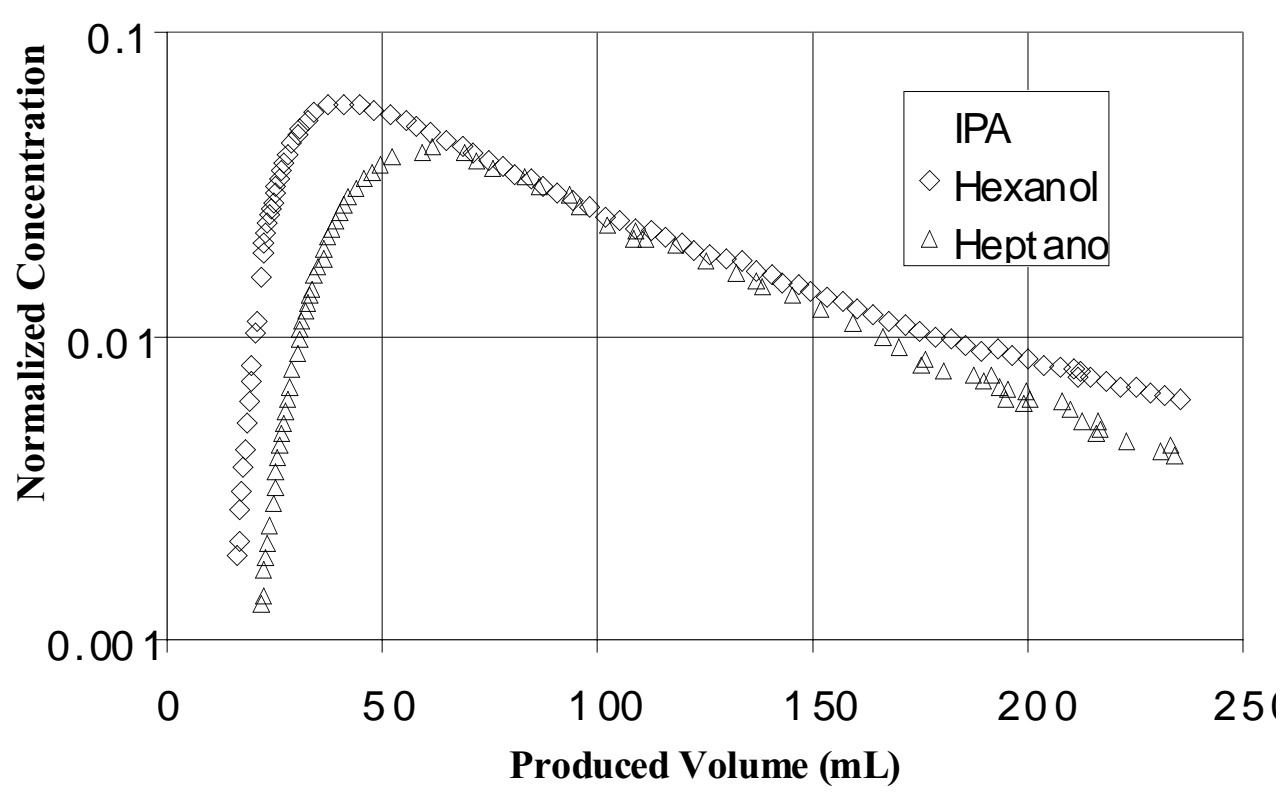

Figure 5.29: Tracer response data for Frac11. 


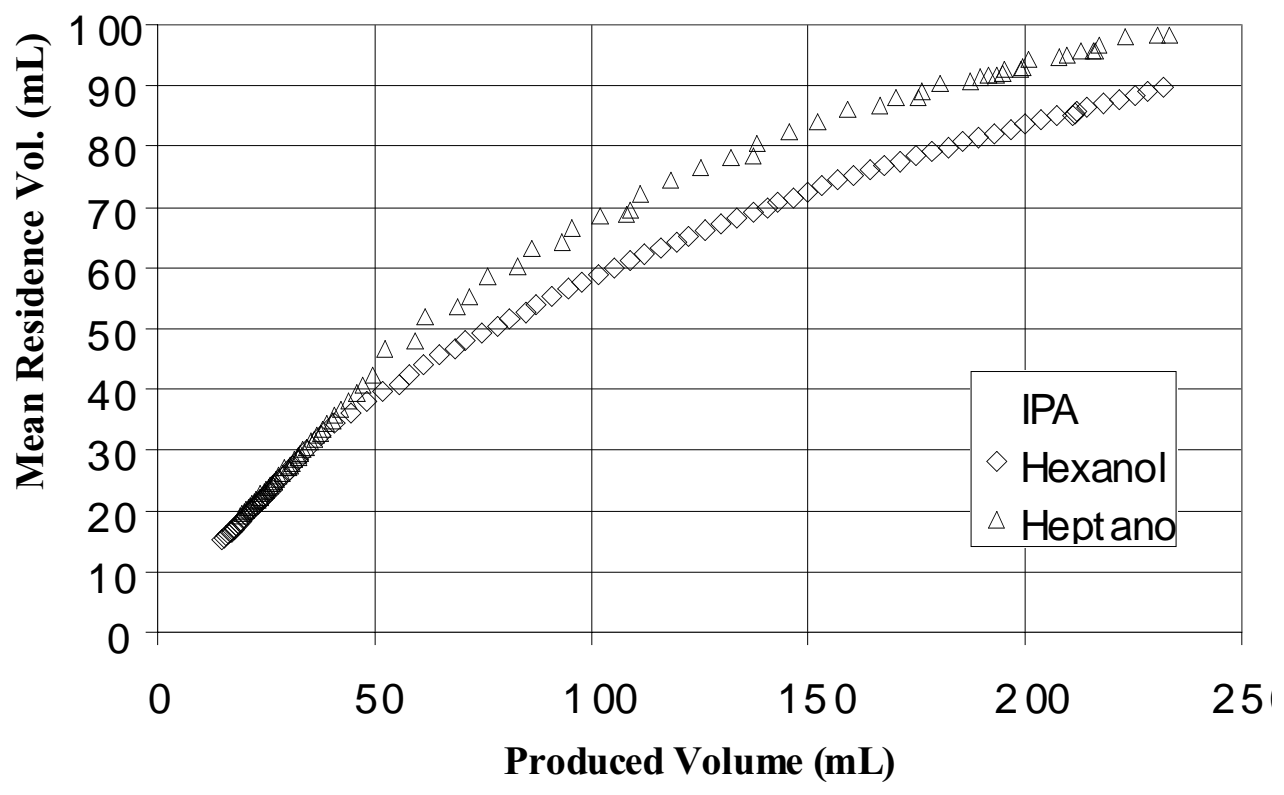

Figure 5.30: Cumulative mean residence volumes, Frac11.

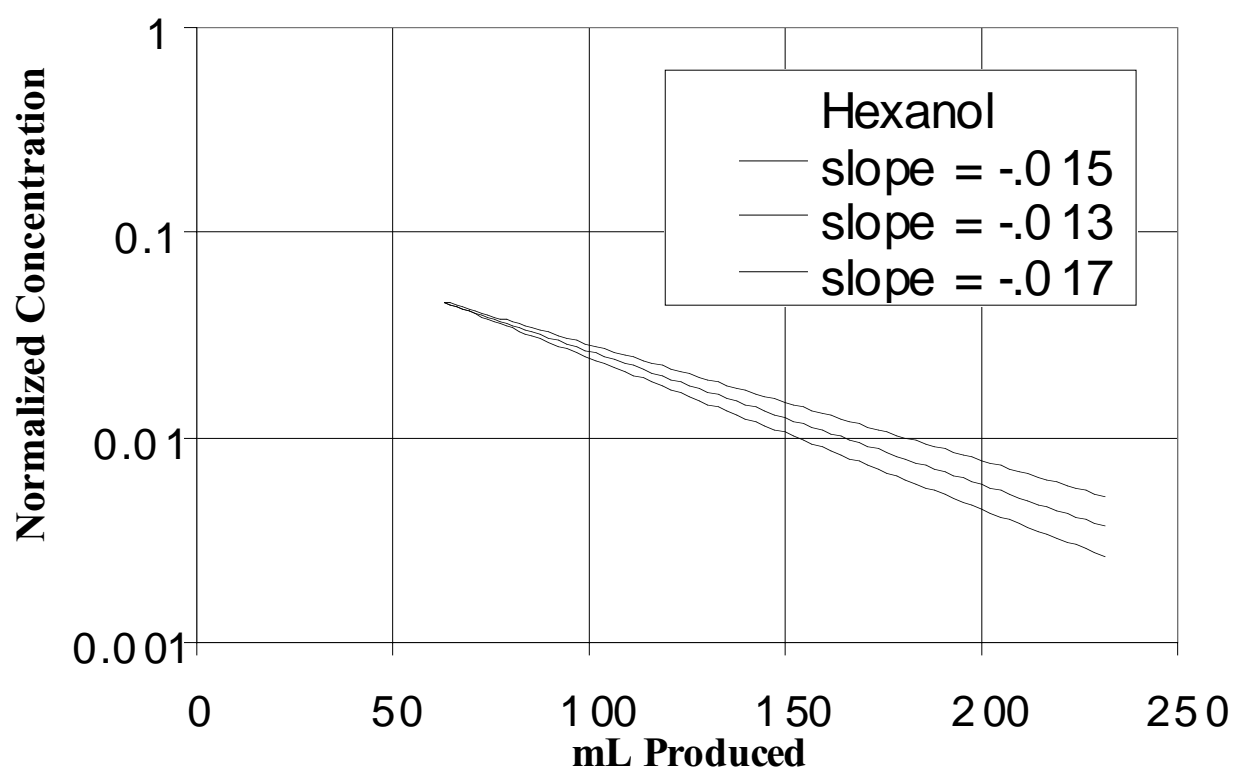

Figure 5.31: Early time extrapolated lines for sensitivity analysis. 


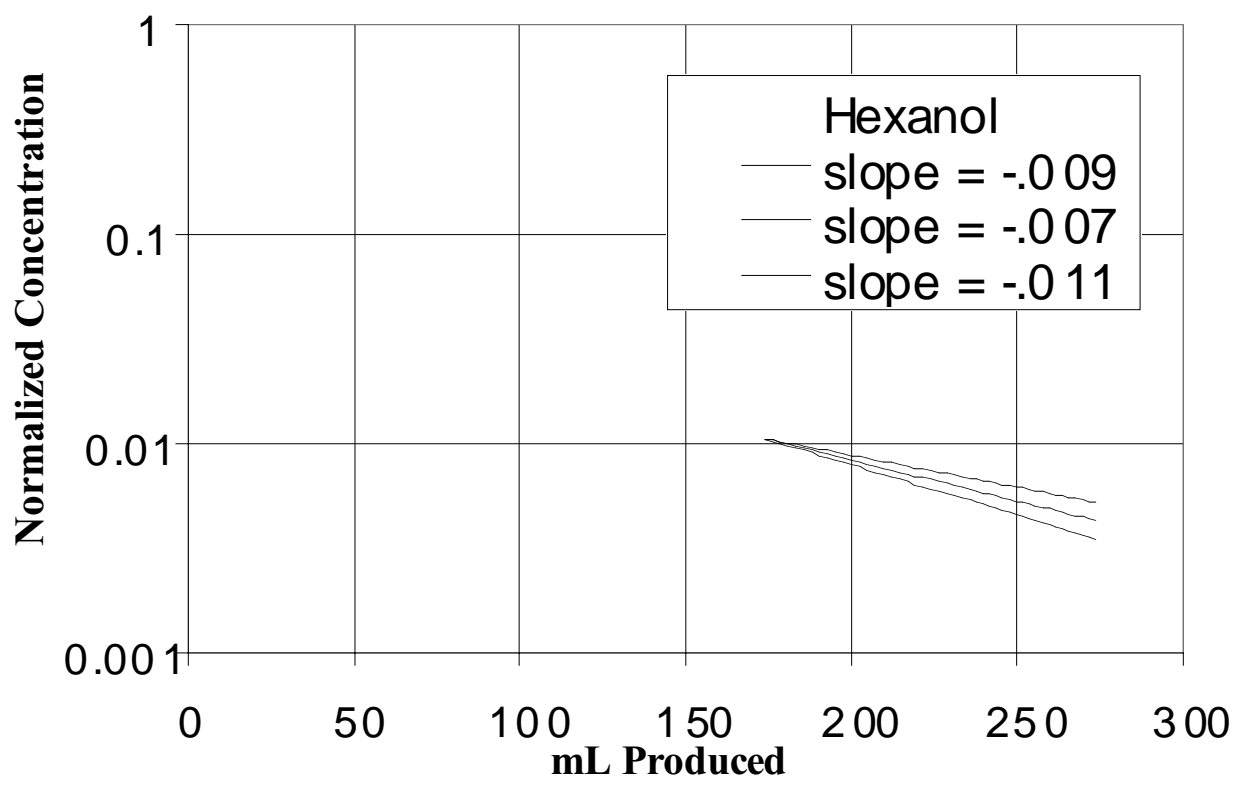

Figure 5.32: Late time extrapolated lines for sensitivity analysis.

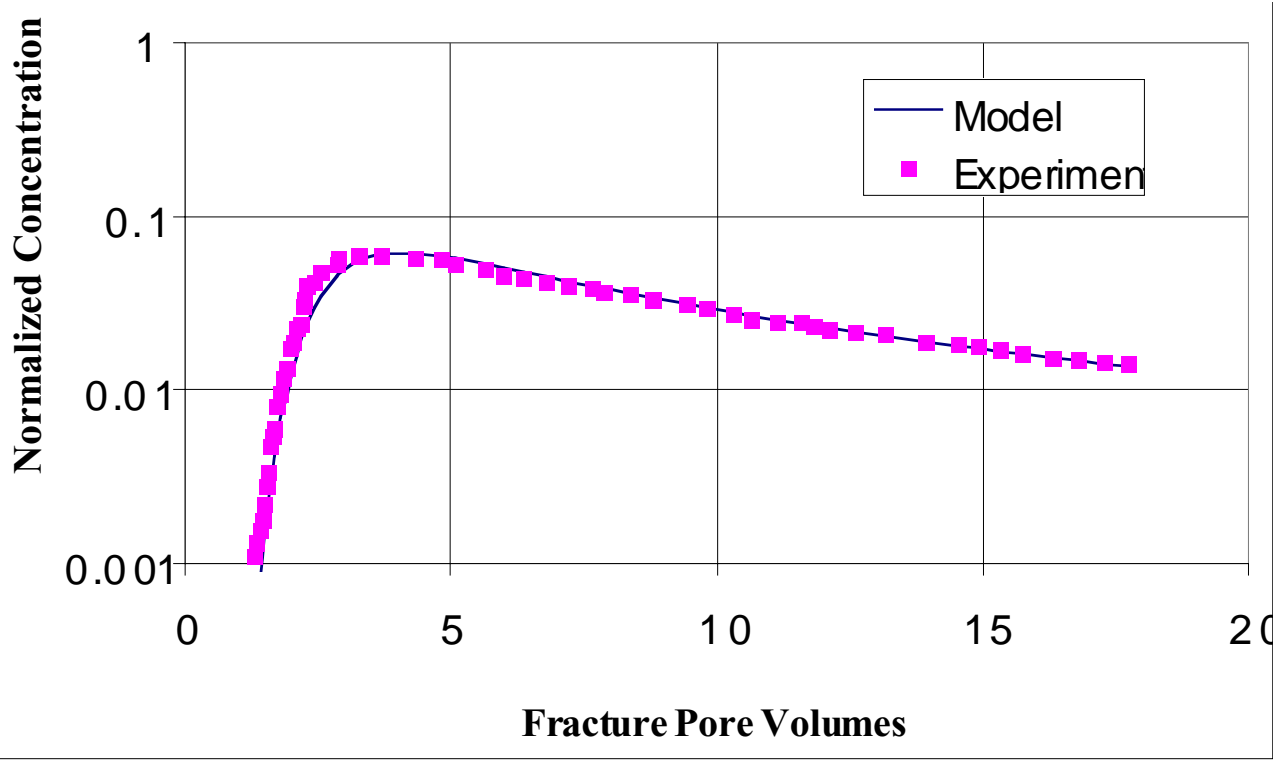

Figure 5.33: IPA experimental data and model fit, Frac11. 


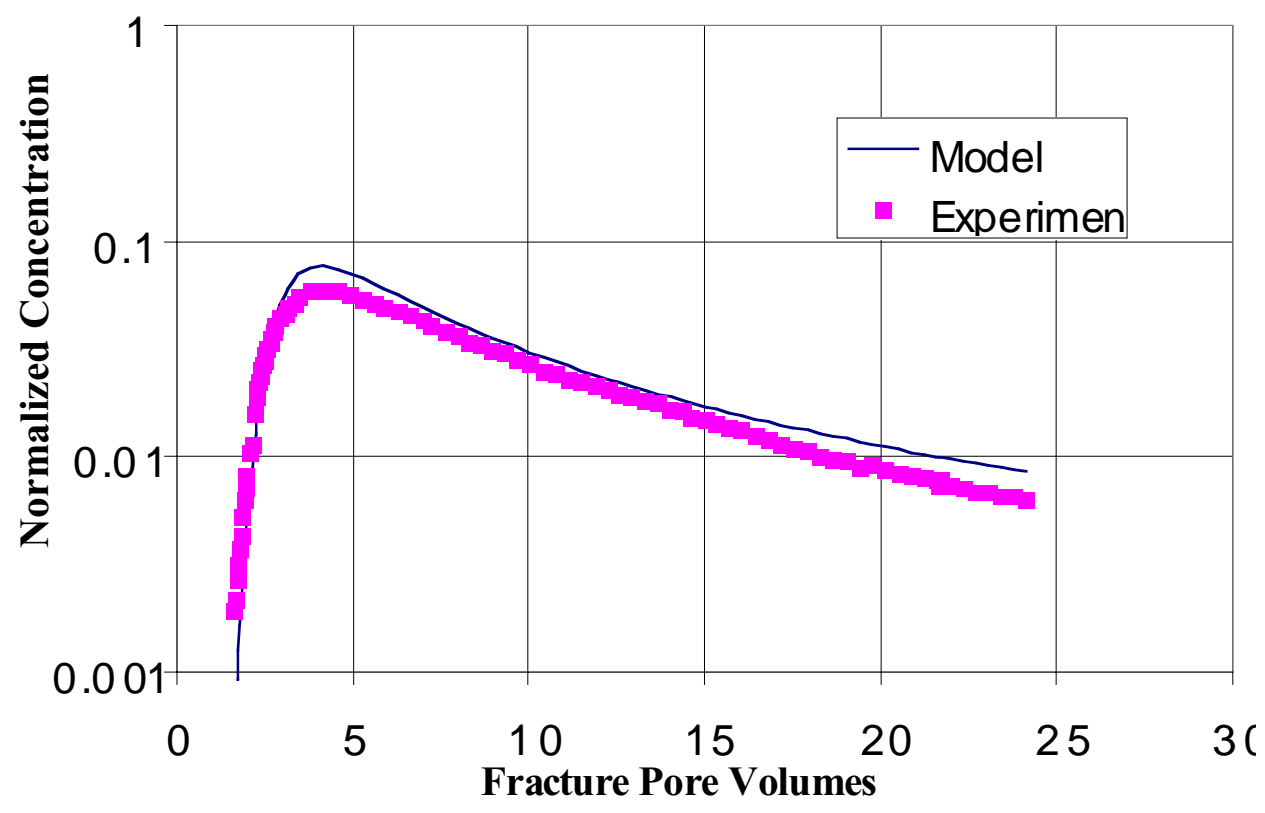

Figure 5.34: Hexanol experimental data and model fit, Frac11.

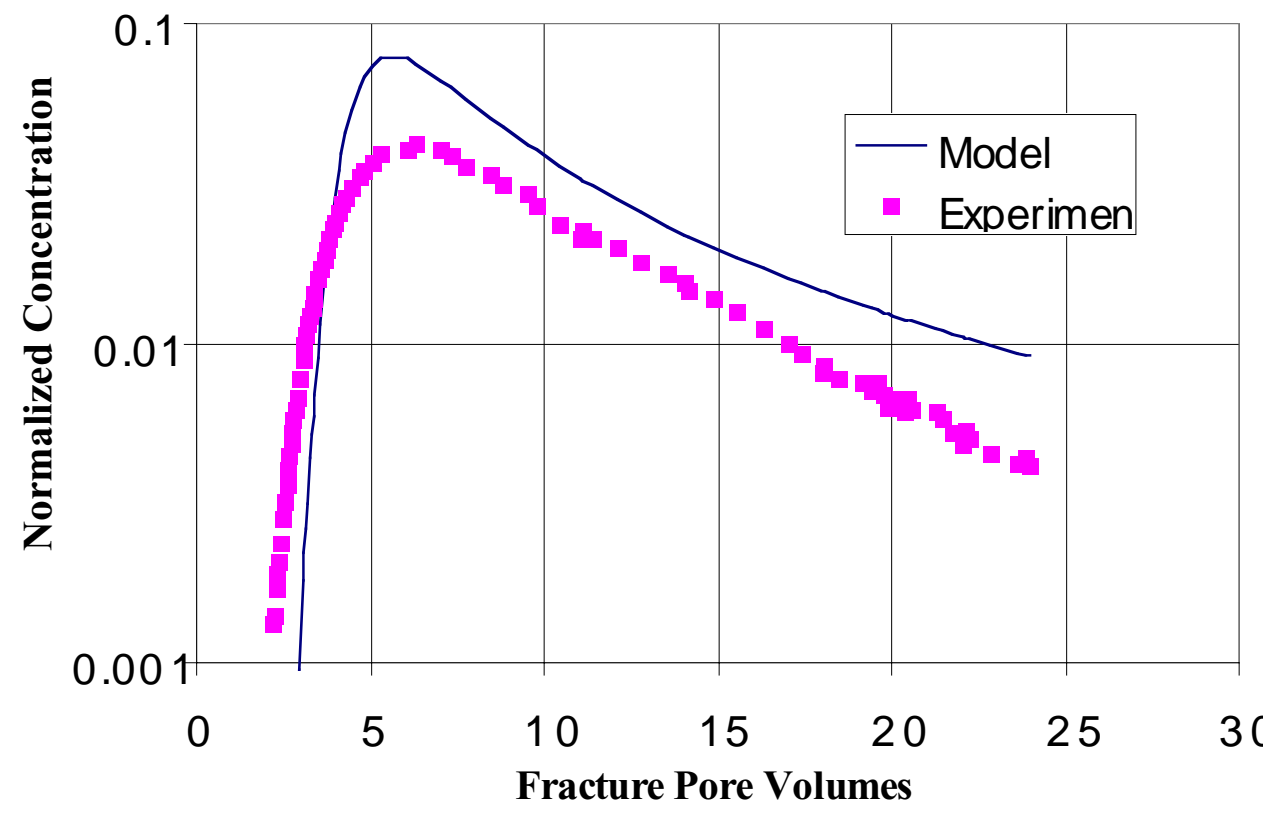

Figure 5.35: Heptanol experimental data and model fit, Frac11. 


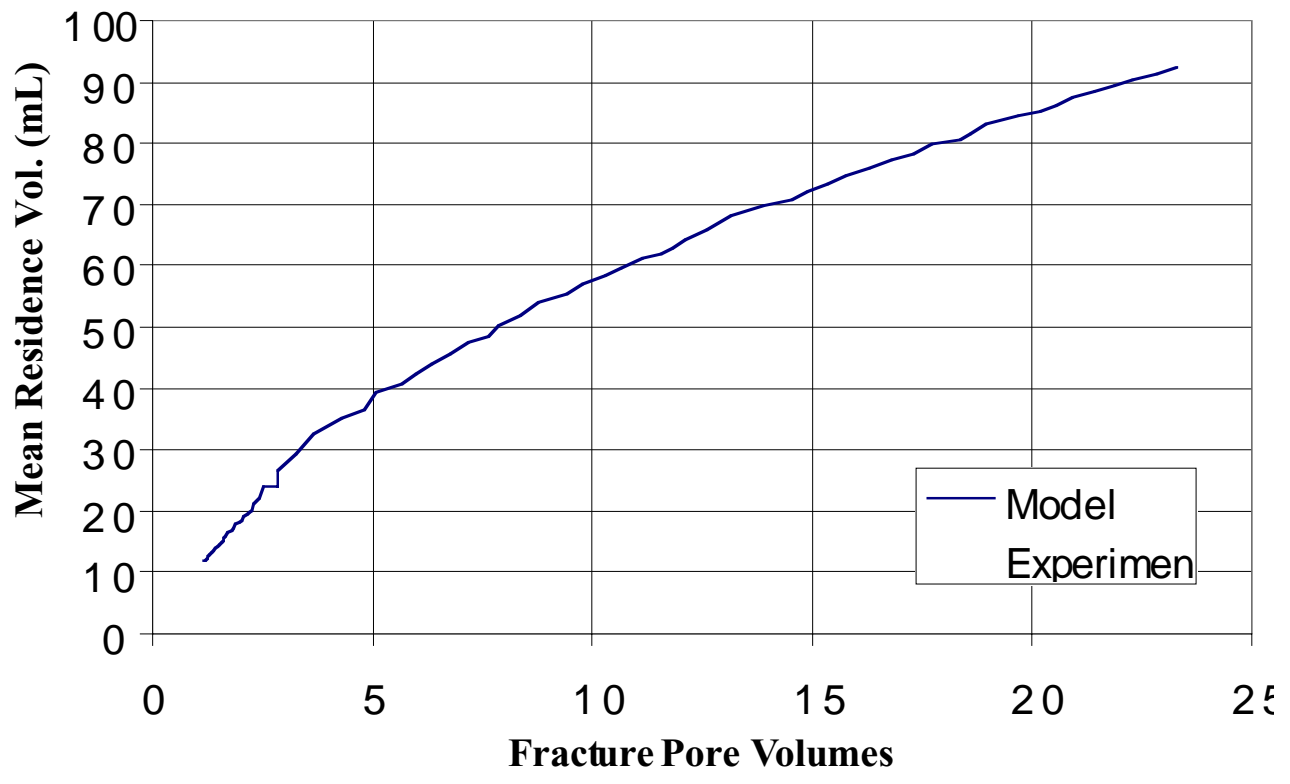

Figure 5.36: IPA cumulative MRV and model fit, Frac11.

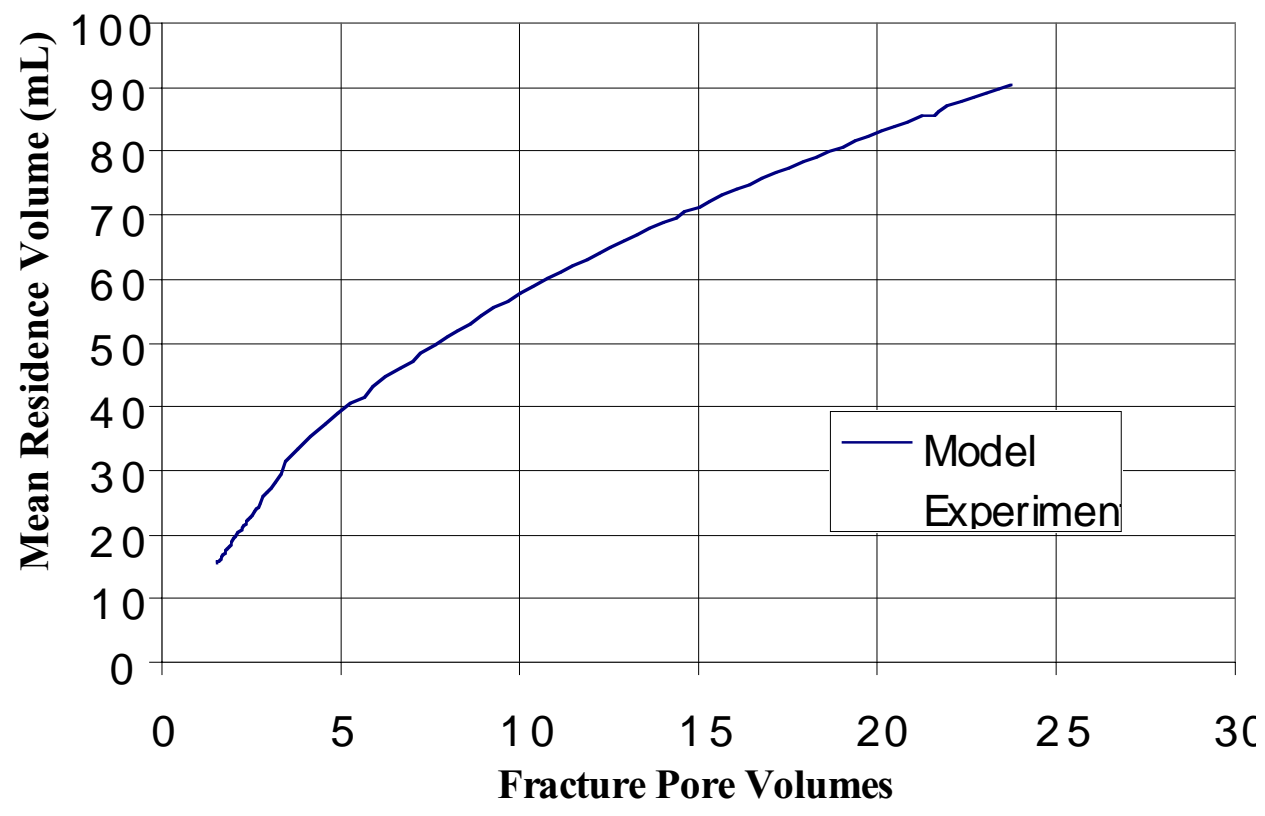

Figure 5.37: Hexanol cumulative MRV and model fit, Frac11. 


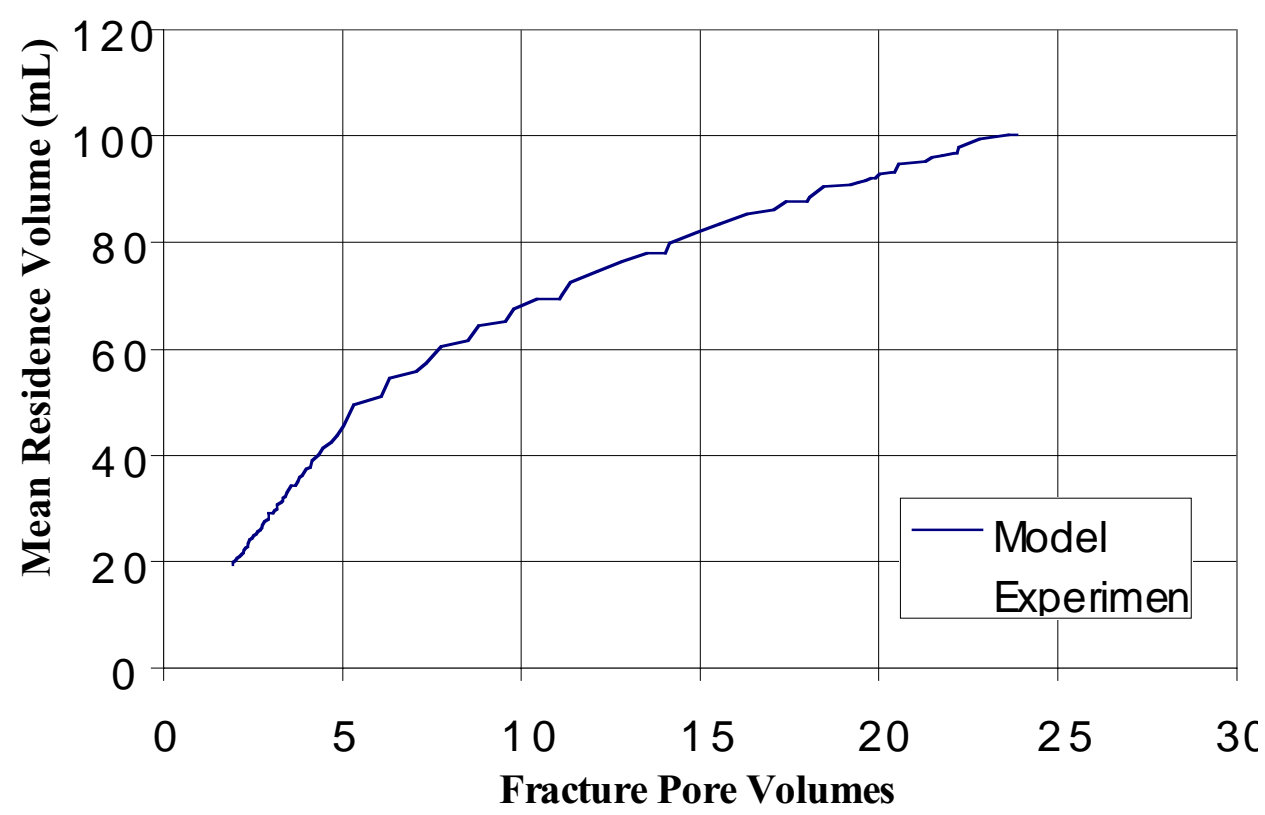

Figure 5.38: Heptanol cumulative MRV and model fit, Frac11.

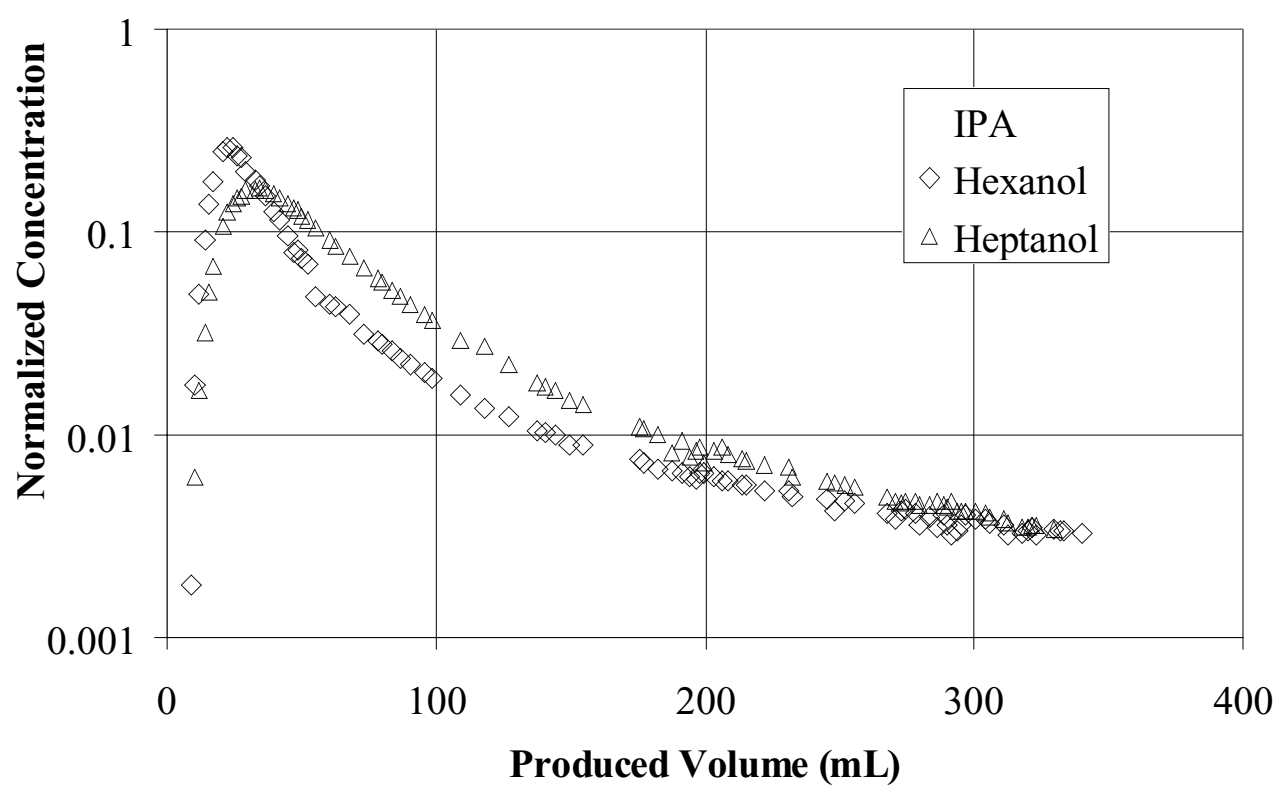

Figure 5.39: Tracer concentration response data for Frac12. 


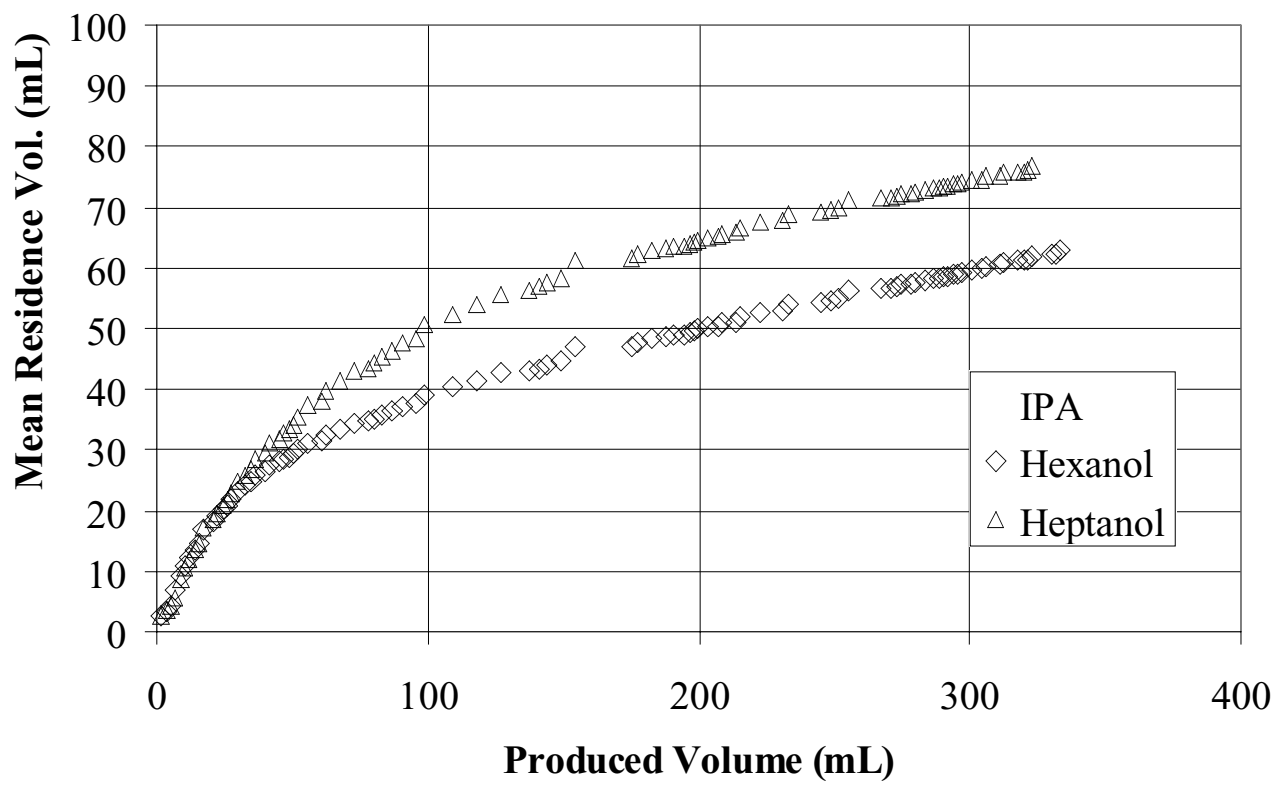

Figure 5.40: MRV data for experiment Frac12.

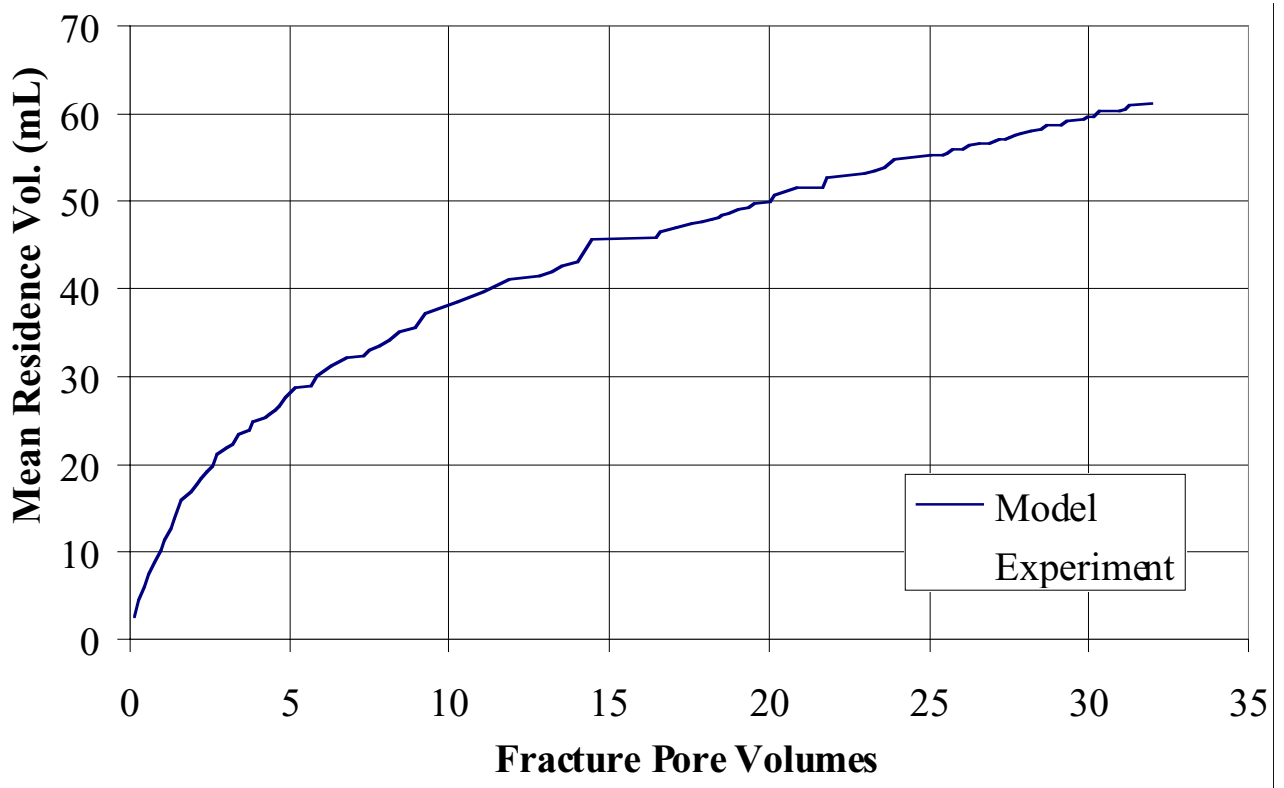

Figure 5.41: MRV data and model fit for IPA, Frac12. 


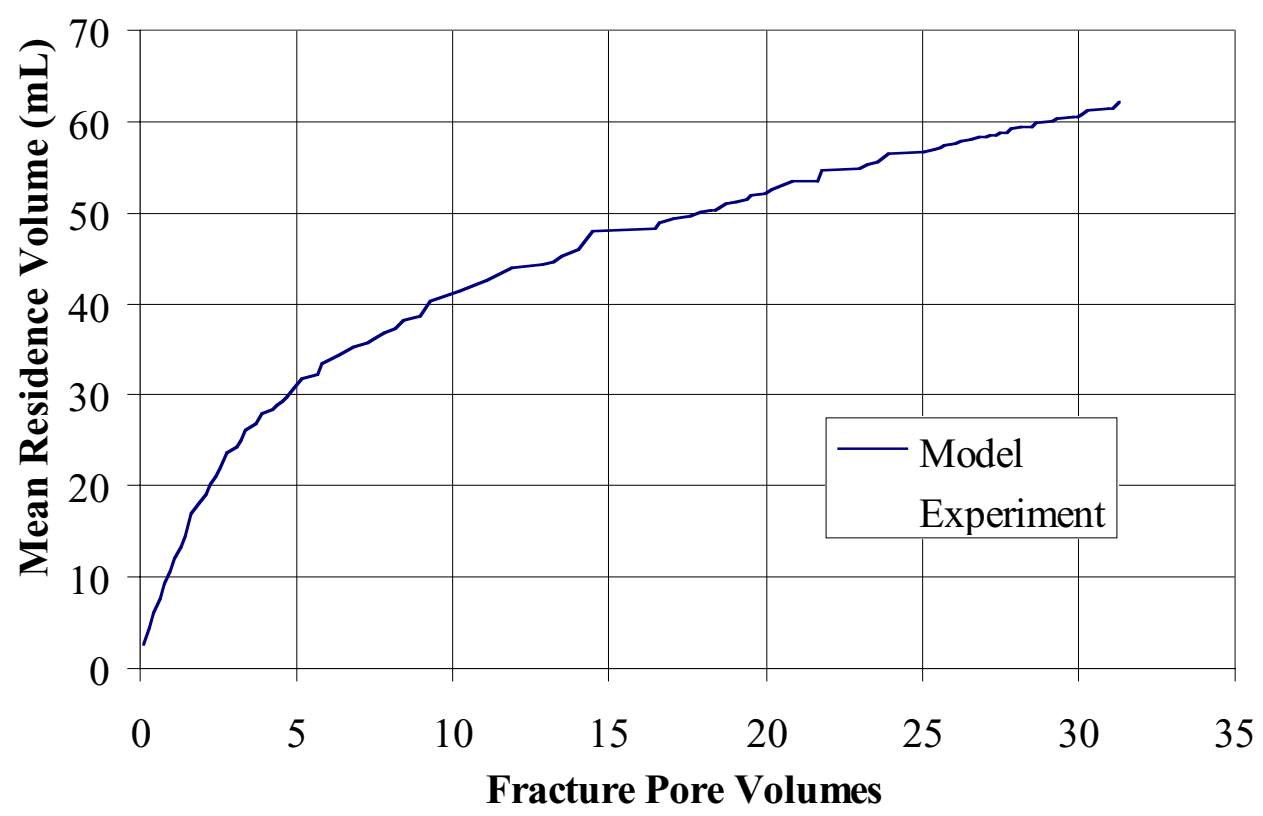

Figure 5.42: MRV data and model fit for hexanol, Frac12.

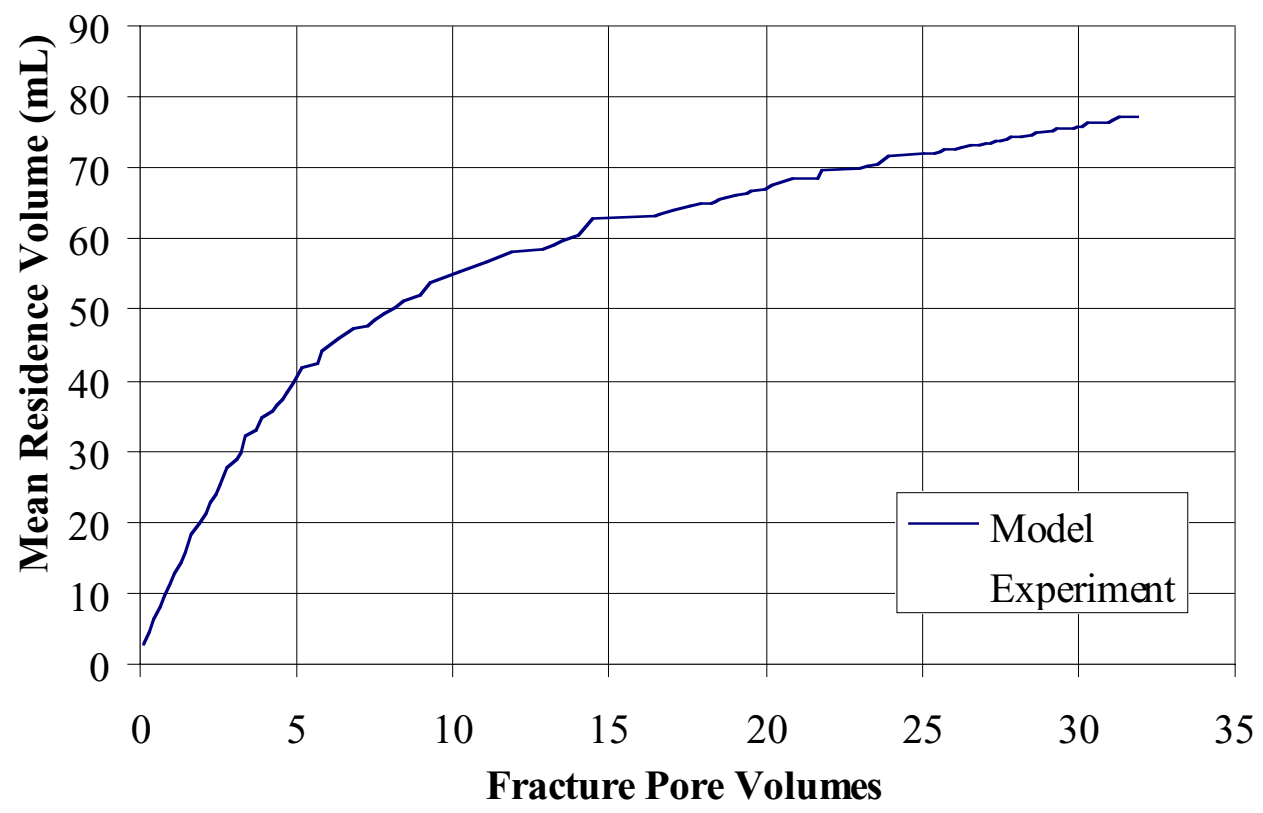

Figure 5.43: MRV data and model fit for heptanol, Frac12. 



\section{Section 6: Three-Dimensional Transport Simulations}

To help evaluate PITT technology at the field scale, UTCHEM (Delshad et al., 1996) was used to model 3-D tracer transport in fractured media. This section contains a general description of how UTCHEM simulates flow in fractured media. In addition, this section contains 3-D UTCHEM simulations of NAPL spills and field scale PITTs in fractured media.

\subsection{The Dual Porosity Model}

UTCHEM simulates tracer flow in fractured media by approximating the fractured rock as a dual porosity system (EPA, 1999; Abdulaziz, 1999). A dual porosity model of fractured media assumes that there are two distinct transport systems: an interconnected fracture system and a disjoint matrix system. This concept is illustrated in Fig. 6.1 using cubic matrix blocks (the socalled sugar cube model.) The approach is analogous to the 1-D parallel fracture model described in Section 4, extended to three dimensions.

The important characteristics of the dual porosity approach are as follows:

1. Advective-dispersive flow and transport are assumed to occur only in the interconnected fractures, which occupy a small fraction of the total volume but are characterized by high permeability.

2. Transport in the matrix blocks is by diffusion only.

3. The two transport systems exchange mass by means of a transfer function.

The equations used in the UTCHEM model can be found in EPA (1999).

\subsection{Subgridding}

The UTCHEM dual-porosity feature adds additional subgridding to the main finite difference grid used for porous media problems. The matrix blocks are divided into smaller sections, so 
that the diffusive transport within the blocks can be modeled accurately. Matrix blocks are divided into parallelepipeds for horizontal diffusion and into slabs for vertical diffusion, as illustrated in Fig. 6.2.

As with any numerical solution, we must determine the number of gridblocks (or in this case subgrids) required to yield a result of acceptable accuracy. EPA (1999) indicates that the solution converges with 8 evenly spaced horizontal subgrids. However, the case presented uses a relatively long test duration (2000 days) and a high matrix diffusion coefficient $\left(4.32 \times 10^{-3}\right.$ $\mathrm{ft}^{3} /$ day, which ensures penetration of the tracer to the center of the matrix blocks. For a case where the test duration is short, or the diffusion coefficient is small, the tracer would penetrate only a fraction of the distance to the center of the matrix block. For the case of incomplete penetration of the tracer into the matrix block, only the outside subgrids will be involved in meaningful calculations. If the subgrids are evenly spaced, then the inner subgrids only serve to increase the CPU time required for the problem.

Ideally the number and location of subgrids used in each simulation should be sufficient for an accurate result, yet minimized to save computer time. The goal of this effort was to devise a rule of thumb for subgridding, applicable for a wide range of input sets. The approach is based on two main observations:

1. For many PITT scenerios in fractured media, the majority of the tracer mass will diffuse only a short distance into the matrix blocks. For these cases, some inner volume of the matrix blocks will contain negligible tracer concentrations. A minimum number of very coarse grids should be used where the tracer concentration is nearly zero in the blocks over the course of the run. 
2. Approximately the same number of subgrids should be adequate for any penetration distance, as long as the fine subgrids are concentrated in the volume of interest.

The approach used here was to first determine the necessary subgrid resolution, then determine what portion of the matrix blocks require the refined subgrids. For example, Fig. 6.3 illustrates a concentration distribution in a matrix block, with the fracture on the left side. The concentration decreases to zero near the center of the block. Figure 6.4 shows how this concentration distribution would appear if only two subgrids were used. For this illustrative case, two subgrids do not appear adequate. Figures 6.5 and 6.6 show the subgrid representations for 4 and 20 subgrids, respectively. Note that for the 20 subgrid case, the concentration distribution is relatively smooth across the entire width of the block. In general, a finer grid resolution yields a more accurate representation of the concentration distribution in the matrix block.

Figure 6.6 also illustrates the problem with using equally spaced subgrids. Ten of the twenty subgrids contain nearly zero tracer concentration, and will have little effect on the calculations, except for increasing CPU time. Figure 6.7 illustrates how the inner fine subgrids can be changed to a single coarse subgrid, without loss of accuracy, since the coarse subgrid has nearly zero tracer concentration.

For Fickian-type diffusion, the concentration distribution of the solute is assumed to be normal, and

$$
\sigma=\sqrt{2 D_{m} t}
$$

This expression for the standard deviation of the concentration distribution is a convenient way to express the tracer penetration distance for a solute with a matrix diffusion coefficient $D_{m}$ at simulation time $t$. One standard deviation should contain about $67 \%$ of the tracer mass, and two 
standard deviations should contain over $95 \%$ of the tracer mass. Note that a typical solution for the Fickian expression is given in the literature as (i.e., Sahimi, 1995)

$$
\text { penetrationdis tan ce }=\sqrt{4 D_{m} t}=1.41 \sqrt{2 D_{m} t}
$$

which should contain approximately $85 \%$ of the tracer mass. Equation 6.2 is derived from a solution to the Fickian diffusion equation with a constant concentration boundary condition (i.e., Fetter, 1993).

Multiple UTCHEM 1-D runs were made to first determine the required subgrid resolution. The resolution of the subgrids is expressed in terms of grids per standard deviation, so that the results can be scaled for any problem. The standard deviation was calculated from equation (6.1), with $t$ being equal to the estimated test duration. The runs were designed so that significant tracer diffusion into the matrix blocks would occur, yet without tracer mass reaching the center of the blocks. The vertical and horizontal subgrids were tested separately, with the diffusion coefficient in the orthogonal direction set to zero for each case.

Figure 6.8 shows the conservative tracer response curves for varying vertical subgrid resolutions. All subgrids were spaced evenly for these initial runs. Figure 6.9 shows the partitioning tracer response curves for the same runs, where $R_{f}$ was 2.1. Both figures show that the response curves converge visually at 50 total subgrids, or 4.5 subgrids $/ \sigma$.

UTCHEM runs were then made with 4.5 subgrids $/ \sigma$ from the surface to a specified depth into the matrix blocks. Figure 6.10 shows that the conservative tracer response curves converged when 4.5 subgrids $/ \sigma$ were used to a depth of $1.5 \sigma$. Figure 6.11 shows a similar result for the partitioning tracer response curves. The final result was a requirement of approximately 7-8 subgrids concentrated in the area between the surface of the matrix block and $1.5 \sigma$, where the standard deviation is defined as equation (6.1). 
A similar process was completed with the horizontal subgrids. Figures $6.12-6.15$ show the tracer response curves for these UTCHEM runs. The results from the analysis of the horizontal subgrids was a similar requirement of approximately 7-8 subgrids concentrated on the outside edge of the matrix block to a distance of $1.5 \sigma$.

If the input parameters for the PITT indicate that the tracer would diffuse to the center of the matrix block over the course of the test, then the 8 subgrids should be evenly spaced over the enter matrix block.

\subsection{Modeling a DNAPL Spill}

\subsubsection{Generating a Saturation Distribution}

Simulation of PITTs in fractured media requires some modeled distribution of NAPL in the test zone. In general, one of three methods could be used to create this distribution:

1. Assume a constant NAPL saturation throughout the test zone. One of the interesting characteristics of fracture flow is preferential transport in zones of high permeability. If the NAPL saturation is spatially constant, then the tracer is sure to encounter at least some NAPL regardless of the flow regime, which is unrealistic.

2. Assume some stochastic saturation field, with a normal or lognormal distribution, mean, variance and correlation length. This approach would be convenient, and probably more realistic than assuming a constant spatial saturation. However, no known data exists in the literature that provides evidence of the typical distribution, variance, or correlation length for NAPL saturations at the laboratory or field scales.

3. Simulate the spill using the multiphase flow and transport capabilities of UTCHEM. This option is best because it will generate a NAPL distribution that is consistent with the 
fractured media characteristics chosen for the ensuing PITT. Also, simulating the spill requires fewer a priori assumptions than either options 1 or 2 .

Note that using the same code to simulate the DNAPL spill and PITT tests could hide possible errors, since any problems in the code could be self-correcting. However, UTCHEM is a very well-established multiphase transport simulator which contains no known errors.

\subsubsection{Capillary Pressure in Fractured Media}

UTCHEM has several options for simulating the capillary pressure during multiphase flow, including imbibition Corey, first drainage Corey, or a modified Parker and Lenhard s model. Ideally, Parker and Lenhard s model would be used, because this model accounts for the effects of hysteresis during the spill and subsequent waterflood. However, stability problems were encountered when using this option. Therefore, the spill and waterflood were treated as two separate events. The first drainage Corey option was used for the spill event, and imbibition Corey was used for the subsequent waterflood.

The fractured medium is assumed to be strongly water-wet. The equations used to model two-phase capillary pressure in UTCHEM with the Corey options are given below. First, for the drainage case, capillary pressure $\left(P_{c}\right)$ is calculated by

$$
\frac{P_{b}}{P_{c}} y^{\lambda_{d}}=S_{d}
$$

where

$$
S_{d}=\frac{S_{w}-S_{w r}}{1-S_{w r}}
$$

and $S_{w r}$ is the residual water saturation, and $\lambda_{d}$ is a constant that is a function of media characteristics that can be estimated from experimental data. 
For the imbibition case,

$$
\frac{P_{b}}{P_{c}} \sqrt[V]{ }^{\lambda_{i}}=1-S_{i}
$$

where

$$
S_{i}=\frac{S_{w}-S_{w r}}{1-S_{w r}-S_{N r}}
$$

and $S_{N r}$ is the residual NAPL saturation. Note that in UTCHEM, the $\lambda$ value for both the drainage and imbibition cases is input as $E P C$, the negative reciprocal, i.e.,

$$
E P C=-\frac{1}{\lambda}
$$

For equations (6.3) and (6.5), the entry pressure $\left(P_{b}\right)$ is calculated in UTCHEM from

$$
P_{b}=C P C \sqrt{\frac{\phi}{k}}
$$

where $C P C$ is a constant that can be determined from experimental data. Equation (6.8) is derived from a capillary tube model for porous media.

In fractured media, the permeability in a fracture is most often assumed to vary as the square of the aperture width (i.e., Kazemi et al., 1995)

$$
k=F_{R} \frac{(2 b)^{2}}{12}
$$

where $F_{R}$ is a roughness factor that is assumed equal to 0.6 for natural fractures. Note that $b$ is the half aperture width, to keep the notation consistent with Sections 4 and 5. The entry pressure for a rough-walled fracture is approximated by (Kueper and McWhorter, 1991):

$$
P_{b}=\frac{\sigma \cos \theta}{b}
$$

where 


$$
\begin{aligned}
& \theta=\text { contact angle } \\
& \sigma=\text { water/NAPL interfacial tension }
\end{aligned}
$$

Substituting equation (6.9) into (6.10) yields

$$
P_{b}=\frac{\sqrt{0.6} \sigma \cos \theta}{\sqrt{3}} \frac{1}{\sqrt{k}}
$$

Equation (6.11) shows that for the assumptions made here, the entry pressure for a fracture should scale as $\frac{1}{\sqrt{k}}$, instead of $\sqrt{\frac{\phi}{k}}$ as given for porous media in equation (6.8). A simple modification was made to UTCHEM to account for this difference in the scaling of $P_{b}$, so that

$$
P_{b}=C P C \frac{1}{\sqrt{k}}
$$

The $\lambda$ parameters in equations (6.3) and (6.5) were determined by fitting these equations to experimental capillary pressure data for a fractured rock from Reitsma and Kueper (1994). The fractured rock described in this paper was a $29.5 \times 26.5 \times 12 \mathrm{~cm}$ dolomitic limestone sample, with the single fracture plane parallel to the $29.5 \times 26.5 \mathrm{~cm}$ face. The fracture surfaces are described as competent and clean, with an obviously rough texture.

Reitsma and Kueper (1994) report estimates for $\lambda_{d}$ and $P_{b}$ for drainage curves in fractures with two different apertures. However, although data for the imbibition case was provided in the paper, no attempt was made by the authors to model the curve. The imbibition data was read from the chart provided, and equation (6.5) was fit using the Excel solver. The scaling constant, $C P C$, for UTCHEM was then calculated for both the drainage and imbibition cases using equation (6.11). Table 6.1 gives a summary of the capillary pressure parameters for the DNAPL spill simulation, as well as the corresponding UTCHEM input parameters. The 
DNAPL used in the simulations had the same interfacial tension (IFT), at $27 \mathrm{dyn} / \mathrm{cm}$, as the NAPL used in Reitsma and Kueper (1994). The density of the simulated DNAPL was $1.3 \mathrm{~g} / \mathrm{mL}$ and the viscosity was $0.6 \mathrm{cP}$. These values of IFT, density, and viscosity are similar to those reported for pure 1,1,1-trichloroethane (TCA) (Jackson and Dwarakanath, 1999). Note that for actual field DNAPL, the IFT is often much lower due to impurities such as grease (Jackson and Dwarakanath, 1999).

\subsubsection{Simulation Volume Characteristics}

The test volume had dimensions of $20 \times 9 \times 10 \mathrm{ft}(6.1 \times 2.7 \times 3.0 \mathrm{~m})$, with $1 \mathrm{ft}(0.3 \mathrm{~m})$ square gridblocks as shown in Fig. 6.17. The DNAPL spill cell was located at the top layer of the grid, centered in the $\mathrm{x}-\mathrm{y}$ plane.

A stochastic fracture porosity distribution was created for the test volume. Direct measurements of fracture aperture values in laboratory core samples (Snow, 1970) have shown that the aperture can be represented by a lognormal distribution. Because the matrix blocks are cubes, the fracture porosity can be calculated from the half aperture width, $b$, and the block edge length, $L_{b}$, using the following equation:

$$
\phi_{f}=1-\frac{\left(L_{b}-2 b\right)^{3}}{L_{b}^{3}}
$$

The fracture permeability can also be calculated from the fracture aperture using equation (6.9).

The lognormal distribution of fracture apertures was generated using FFT (Jennings, 1998), an algorithm which generates a standard normal data set with a specified correlation length. The standard normal data set, $z$, was transformed to a lognormal data set with a mean $\mu_{b}$ and a $\log$ standard deviation $\sigma_{\ln (b)}$ with the following equation: 


$$
b_{i}=\mu_{b} \exp \left(\sigma_{\ln (b)}\left(z_{i}-\frac{\sigma_{\ln (b)}}{2}\right)\right)
$$

The mean fracture aperture was chosen for these simulations was $0.01 \mathrm{~cm}$. The $\log$ standard deviation, $\sigma_{\ln (b)}$, was 1.0 , and the correlation length was $10 \mathrm{ft}(3.0 \mathrm{~m})$ in the x- and ydirections, and $1 \mathrm{ft}(0.3 \mathrm{~m})$ in the z-direction. The 10:1 ratio of horizontal to vertical correlation lengths tends to give horizontal fractures in preference to vertical fractures. The fracture spacing was $1 \mathrm{ft}(0.3 \mathrm{~m})$, so the average fracture porosity (from equation $(6.14)$ ) was $1.0 \times 10^{-3}$. The average permeability (from equation (6.9)) was $1.7 \times 10^{3}$ Darcies, with a range from 0.84 Darcies to $1.03 \times 10^{5}$ Darcies.

Table 6.2 summarizes the mean values and ranges for all of these parameters. Figures $6.18-6.20$ show the orthogonal midpoint slices from the permeability field, to give a visual perspective of the correlation structure in the volume. Note the strong correlation in the $\mathrm{x}$ - and y-directions, and the weak correlation in the z-direction. Appendix D contains the input file used for the spill simulation, which includes all of the stochastic fields.

\subsubsection{Spill Simulation Results}

Table 6.3 summarizes the well parameters for the spill and waterflood simulations. The DNAPL spill rate was $0.02 \mathrm{ft}^{3} / \mathrm{d}\left(6.6 \times 10^{-9} \mathrm{~m}^{3} / \mathrm{s}\right)$ for 1 day, for a total spilled DNAPL volume of $0.02 \mathrm{ft}^{3}$ $\left(5.7 \times 10^{-3} \mathrm{~m}^{3}\right)$, which gives an average DNAPL saturation of 0.01. Figures 6.21-6.24 show the $S_{N}=0.01$ isosurface at various times during the spill event. Although the average permeability in the volume is very high, some trapping and spreading of the DNAPL occurs, due to the highly heterogeneous permeability field. This simulation indicates that in fractured systems, where the fracture permeability is relatively high compared to typical porous media, the DNAPL may still be trapped due to abrupt variations in the permeability. 
After the 1 day spill event, two wells were added--one at each end of the x-axis, centered on the y-axis and screened throughout the thickness. For 1 day, water was injected at a rate of $2.0 \mathrm{ft}^{3} / \mathrm{d}$ (approximately 1 pore volume per day) in the injection well (Fig. 6.17) and extracted at the same rate from the extraction well. Figure 6.25 shows the $S_{N}=0.01$ isosurface after 0.1 days of water flooding. The trapped DNAPL is smeared slightly in the positive x-direction, and some of the DNAPL is mobilized vertically downward. Figure 6.26 shows that very little change occurred after the initial 0.1 days of waterflooding. Figure 6.27 shows a comparison of the DNAPL saturations projected in the $\mathrm{x}-\mathrm{z}$ plane, before and after the waterflood. Before the waterflood, the DNAPL was trapped in some cells at a relatively high saturation. After the waterflood, the DNAPL has been spread to a lower saturation over a larger volume. Figure 6.27 shows that after the waterflood, although the DNAPL migrated vertically down, significant pooling did not occur at the bottom boundary for this case.

Frequency histograms were plotted for the DNAPL saturations before and after the waterflood. Only cells that had a positive saturation were included in the data set. Figure 6.28 shows the saturation histogram before the waterflood. This histogram shows that a significant number of cells had relatively high saturations (i.e., $>0.10)$, and a wide range of saturations $(0-$ 0.65). Figure 6.29 shows the saturation histogram after the waterflood. Note the difference in the saturation scale for this figure. After the waterflood, the range of saturations is much smaller $(0-0.16)$, with the highest frequencies at the ends. These histograms indicate that fitting a typical distribution model to the saturation data would be difficult, especially after the waterflood. 


\subsection{PITT Simulations}

Partitioning interwell tracer test simulations used the results from the DNAPL spill/waterflood of section 6.3. The same permeability and porosity fields were used (as summarized in Table 6.2). The same well locations (summarized in Table 6.3) for the injector and producer pair were used. Table 6.4 gives the partitioning tracers used in the simulations, with their partition coefficients and diffusion coefficients. The tracers were chosen to provide a recommended range of retardation factors $\left(1.2<R_{f}<3.0\right)$ given the known NAPL saturation.

For the PITT in fractured media simulations, the parameters that were varied were the porosity of the matrix and the diffusion coefficients of the tracers in the matrix. The diffusion coefficients in the matrix $\left(D_{m}\right)$ were calculated from

$$
D_{m}=D_{w} \tau
$$

where $\tau$ is the same tortuosity defined in section 5.2.3, and $D_{w}$ is the tracer diffusion coefficient in water (Table 6.4). Experimental data are available that relate the tortuosity of a given consolidated porous medium with the porosity. Donaldson et al. (1976) performed experiments to determine the tortuosity in sandstone cores. Some of the results are presented in Table 6.5. Reported values in Neretnieks (1993) for granite and gneiss with and without fissure coatings vary over several orders of magnitude. A range of tortuosity values for a range of porosities are given for granite and gneiss in Table 6.5. The values for tortuosity used in the simulations were $\tau$ $=0.001$ for $\phi_{m}=0.01$ and $\tau=0.03$ for $\phi_{m}=0.1$.

\subsubsection{Simulation TT035 - No Matrix Diffusion}

In the first PITT simulation (TT035), the effect of matrix diffusion was eliminated by setting all of the diffusion coefficients for the tracers to zero. This simulation was to provide a baseline as 
to the predicted accuracy of the DNAPL volume and saturation estimates calculated from the tracer results. The injection and extraction rate was $2.0 \mathrm{ft}^{3} / \mathrm{d}$ and the tracer slug duration was 0.5 days. The duration of the PITT was one week, a typical duration for field PITTs in porous media. The DNAPL volume and saturation estimates were calculated from the tracer output curves, using equations 2.9 and 2.5 , respectively.

Figure 6.30 shows the tracer response curves for TT035. As expected, the tracer response curves are typical for partitioning tracer tests, with a relative lag of the partitioning tracer with respect to the conservative tracer, and linear tailing on a semi-log plot at late time. Figure 6.31 shows that the mass recovery was nearly $100 \%$ for all of the tracers. Because the tracers were transported in the fractures only, the mass recovery should have been $100 \%$ over the course of the simulation, provided the duration of the PITT was sufficiently long. Figure 6.32 shows the cumulative mean residence volume for each of the tracers. The curves reach a maximum for the 1-propanol and the 2,3-dimethyl-3-pentanol, while the 1-heptanol curve is still increasing when the simulation is terminated. Because the heptanol MRV curve has not reached a maximum, without extrapolation the heptanol data would be expected to underpredict the NAPL volume and saturation.

Figure 6.33 shows that the heptanol tracer does underpredict the NAPL saturation by approximately $20 \%$. If the simulation were carried out for another week, the heptanol curve would converge with the 2,3-dimethyl-3-pentanol, which accurately estimated the NAPL saturation. Figure 6.34 shows the same trends in the NAPL volume prediction. For this case with no matrix diffusion, the NAPL volume and NAPL saturation estimates were of approximately the same accuracy. 


\subsubsection{Simulation TT037 - Minimal Matrix Diffusion}

The input parameters for simulation TT037 were similar to those of TT035, except tracer diffusion into the matrix was included. The matrix porosity for this simulation was 0.01 , and the tortuosity was 0.001 , so the effective diffusion coefficients for the tracers in the matrix were three orders of magnitude less than their bulk diffusion coefficients in water.

Figure 6.35 shows the tracer response curves for TT037. As with TT035, the response curves do not show any significant effect from matrix diffusion. A slight non-linearity exists in the tail of the 1-propanol response curve, but some small numerical instability may also have caused this effect. Figure 6.36 indicates that the mass recoveries were again nearly $100 \%$ for all tracers, so minimal amounts of tracer were retarded in the matrix during the course of the simulation. Figure 6.37 shows the cumulative MRVs for TT037. As with TT035, the 1-heptanol MRV is still increasing at the end of the simulation.

Figure 6.38 shows the DNAPL saturation estimate from the tracer response curves for TT037. In this case, the 2,3-dimethyl-3-pentanol overestimates the NAPL saturation by approximately $3 \%$, while the heptanol underestimates the saturation by approximately $18 \%$. Figure 6.39 shows that the 2,3-dimethyl-3-pentanol data overestimates the NAPL volume by about $8 \%$. This overestimation is puzzling because the opposite effect would be expected if matrix diffusion had some impact on the response curves, as seen in the following section.

\subsubsection{Simulation TT038 - Significant Matrix Diffusion}

Simulation TT038 increases the matrix diffusion effect relative to TT037 by increasing the matrix porosity to 0.1 . A matrix porosity of 0.1 corresponds to a tortuosity of approximately 0.03 . 
Figure 6.40 shows the tracer response curves for TT038. The curves show a significant change from the previous simulations. The effect of matrix diffusion has decreased the separation between the conservative and partitioning tracers, and has increased the tailing of all of the tracer response curves. Figure 6.42 shows trends that are similar to the laboratory results given in Section 5. The MRV of the conservative tracer increases faster than the MRVs of the partitioning tracers at late time, which decreases the NAPL saturation and volume estimates at late time. This trend can be seen in Fig. 6.43, where the saturation estimate increases for 2,3dimethyl-3-pentanol, reaches a maximum, and then decreases at late time. Also, the NAPL saturation estimate is only approximately $35 \%$ of the theoretical value for either partitioning tracer. The NAPL volume estimate is slightly better, at 39\% for 2,3-dimethyl-3-pentanol and $63 \%$ for the heptanol response data.

This simulation indicates that when matrix diffusion occurs, the relative error in the NAPL saturation estimate is higher than the relative error in the NAPL volume estimate. To verify whether this relationship is always true, we write the equations for volume and saturation, with a small positive error associated with each mean residence volume:

$$
\begin{aligned}
S_{N}^{*} & =\frac{\bar{V}_{2}+\Delta \bar{V}_{2}-\bar{V}_{1}-\Delta \bar{V}_{1}}{\left(\bar{V}_{1}+\Delta \bar{V}_{1}\right)\left(K_{N}-1\right)+\bar{V}_{2}+\Delta \bar{V}_{2}} \\
V_{N}^{*} & =\frac{\bar{V}_{2}+\Delta \bar{V}_{2}-\bar{V}_{1}-\Delta \bar{V}_{1}}{K_{N}}
\end{aligned}
$$

Combining (6.17) and (6.18), along with the previous equations for $\mathrm{S}_{N}$ and $V_{N}$ in Section 2 gives

$$
\frac{S_{N}^{*} / S_{N}}{V_{N}^{*} / V_{N}}=\frac{V_{N}}{S_{N}} \frac{S_{N}^{*}}{V_{N}^{*}}=\frac{\bar{V}_{1}\left(K_{N}-1\right)+\bar{V}_{2}}{\bar{V}_{1}\left(K_{N}-1\right)+\bar{V}_{2}+\Delta \bar{V}_{1}\left(K_{N}-1\right)+\Delta \bar{V}_{2}}
$$


The expressions $\bar{V}_{1}\left(K_{N}-1\right)+\bar{V}_{2}$ and $\left(K_{N}-1\right)$ are always positive since the partition coefficients are greater than 1 . We replace these expressions with $A^{+}$and $B^{+}$(indicating positive real numbers) in (6.19) to give

$$
\frac{V_{N}}{S_{N}} \frac{S_{N}^{*}}{V_{N}^{*}}=\frac{A^{+}}{A^{+}+\Delta \bar{V}_{1} B^{+}+\Delta \bar{V}_{2}}
$$

Table 6.6 was constructed using equation (6.20) to show how various positive values of $\Delta \bar{V}_{1}$ and $\Delta \bar{V}_{2}$ affect the relative error in the NAPL saturation estimate compared to the NAPL volume estimate. The conditions are listed in order of decreasing likelihood, assuming that for most cases the partitioning tracer will have a lower diffusion coefficient than the conservative tracer, so $\Delta \bar{V}_{2}$ will be less than $\Delta \bar{V}_{1}$.

\subsubsection{Simulations TT039-TT041 - Increased Flow Rate}

The sensitivity analyses of section 4.3 indicated that with decreasing matrix number, the NAPL saturation estimate improves. The previous simulations in section 6.4 also showed this trend, since increasing the effective matrix diffusion coefficient resulted in poorer NAPL saturation estimates. However, one way to keep the matrix number low when effective matrix diffusion coefficients are high is to increase the velocity of the mobile phase by increasing the flow rate.

Simulation TT040 was identical to TT038 except that the flow rate was increased by a factor of 5 , to $10.0 \mathrm{ft}^{3} / \mathrm{d}\left(3.3 \times 10^{-6} \mathrm{~m}^{3} / \mathrm{s}\right)$. The slug duration was reduced by a factor of 5 so that

the overall normalized injected mass was the same for both simulations. Figures $6.45-6.49$ show the TT040 results compared to the TT038 results. Note that the curves are plotted with respect to fracture pore volumes, rather than days, so that the two simulations can be compared on an equal basis. Figure 6.45 shows the change in the tracer response curves with the increased 
flow rate. The curves peak higher and decrease faster with less tailing, indicating less impact of matrix diffusion. Figure 6.46 shows the cumulative mass recovery curves for 1-propanol and 1heptanol for the two simulations. Note that the mass recovery increases by approximately $20 \%$ for TT040, the simulation with the increased flow rate.

Figure 6.47 shows the cumulative mean residence volume curves for the TT040 and TT038. The MRV curves for propanol for TT040 is much closer to the theoretical MRV of 2.0 $\mathrm{ft}^{3}$, and in general, the TT040 curves show less impact from matrix diffusion. The estimated NAPL saturation for the partitioning tracers for both simulations are shown in Fig. 6.48. The saturation estimate for TT040 from the 2,3-dimethyl-3-pentanol tracer is $80 \%$ of the actual value, compared to only $35 \%$ for TT038. For the 1-heptanol tracer, the saturation estimate was again improved, this time from approximately $35 \%$ to $55 \%$ of the actual value. Figure 6.49 shows the NAPL volume estimates. The NAPL volume estimate for the 2,3-dimethyl-3-pentanol tracer improved significantly for TT040 compared to TT038, with an increase from $39 \%$ to $75 \%$ of the actual value. The estimate of the NAPL volume using the 1-heptanol tracer was only incrementally improved, increasing from approximately $63 \%$ to $65 \%$. Note that the results from the lighter, more diffusive partitioning tracer are improved more significantly with the increased flow rate.

The results from these simulations indicate that, as expected, the NAPL saturation and NAPL volume estimates improved with increasing flow rate (and decreasing matrix number). However, no mass transfer limitations were enabled for these simulations, since the mass transfer correlation implemented in UTCHEM is for porous media, not for fractured media. Therefore, a limit exists as to how much the flow rate could be increased before the partitioning tracers are no longer at equilibrium between the water and NAPL, as shown in section 4.3. Without a mass 
transfer correlation for partitioning tracers in fractured media, it is difficult to determine the reliability of results at higher flow rates. However, if the mean residence time required for equilibrium in fractured media is similar to that for porous media, then that partitioning tracers in the TT040 simulation would be sufficiently close to local equilibrium.

\subsection{Summary and Conclusions}

This section includes the 3-D DNAPL spill and PITT simulations in fractured media. The simulations were completed using UTCHEM with the dual-porosity option. First, the matrix block subgridding in UTCHEM was analyzed to determine the optimum number and placement of subgrids for given simulation parameters. In general, approximately 8 subgrids are required in both directions concentrated in the first 1.5 standard deviations of tracer concentration, calculated using the estimated total PITT duration.

The capillary pressure parameters for the spill were determined from existing experimental data. The DNAPL spill resulted in high trapped saturations (i.e., 0.65) in some zones with very low saturations in other zones. The subsequent waterflood induced a more uniform distribution of the DNAPL with most of the saturations ranging from 0.01 to 0.15 .

The PITTs gave a good estimate of the average DNAPL saturation and DNAPL volume at low matrix porosity and correspondingly low (i.e., 0.01) matrix tortuosity. As the porosity and tortuosity were increased, the DNAPL saturation and volume estimates degraded, with the lighter partitioning tracer response affected more significantly than the heavy partitioning tracer. The NAPL volume estimate from PITT data was shown to be more accurate than the NAPL saturation estimate for typical fractured media conditions.

Increasing the flow rate for the PITT increased the accuracy of both the NAPL saturation and volume estimates. The increased flow rate improved the result for the lighter, more diffusive 
partitioning tracer more significantly than for the heavier partitioning tracer. In general, the flow rate should be increased as much as possible without causing non-equilibrium effects with respect to the partitioning of the tracers between the NAPL and water. Because no mass transfer correlation exists for partitioning tracers in fractured media, it was not possible to directly determine at what flow rate non-equilibrium tracer partitioning would cause significant error in the NAPL volume estimates in fractured media.

\subsection{Tables}

Table 6.1: Capillary Pressure Parameters for DNAPL Spill Simulation

\begin{tabular}{|llll|}
\hline & \multicolumn{1}{c}{ Units } & Drainage & Imbibition \\
\hline$P_{b}$ & cm water & 5.12 & 12.0 \\
$\lambda$ & & 1.97 & -0.40 \\
& & & \\
$2 b$ & $\mu \mathrm{m}$ & 59 & 59 \\
$k$ & Darcies & 174 & 174 \\
$C P C$ & psi(Darcies) $^{0.5}$ & 1.0 & 2.3 \\
$E P C$ & & -0.51 & 2.5 \\
\hline
\end{tabular}

Table 6.2: Summary of Common Input Parameters for UTCHEM Simulations SP031, TT035, TT037, TT038, and TT039

\begin{tabular}{|c|c|c|c|c|}
\hline & $\mathbf{x}$ & $\mathbf{y}$ & $\mathbf{z}$ & \\
\hline Grid (blocks) & 20 & 9 & 10 & \\
\hline Gridblock & 1 & 1 & 1 & \\
\hline \multirow{4}{*}{$\begin{array}{l}\text { Dimensions (ft) } \\
\text { Matrix block } \\
\text { Dimensions (ft) }\end{array}$} & & & & \\
\hline & 1 & 1 & 1 & \\
\hline & & & & \\
\hline & mean & $\max$ & $\min$ & $\sigma_{\ln ()}$ \\
\hline Aperture $(\mathrm{cm})$ & 0.01 & $1.44 \mathrm{E}-01$ & $4.11 \mathrm{E}-04$ & 1.0 \\
\hline Fracture Porosity & 0.001 & $1.41 \mathrm{E}-02$ & 4.04E-05 & 1.0 \\
\hline \multirow{2}{*}{$\begin{array}{l}\text { Permeability } \\
\text { (Darcies) }\end{array}$} & $1.67 \mathrm{E}+03$ & $1.03 \mathrm{E}+05$ & 84.3 & 2.0 \\
\hline & Density & Viscosity & $\begin{array}{l}\text { Interfacial } \\
\text { Tension }\end{array}$ & \\
\hline$\overline{\text { DNAPL }}$ & $1.3 \mathrm{~g} / \mathrm{mL}$ & $0.6 \mathrm{cP}$ & $27 \mathrm{dyn} / \mathrm{cm}$ & \\
\hline
\end{tabular}


Table 6.3: Summary of Well Locations and Rates for SP031, a Simulated DNAPL Spill and Waterflood.

\begin{tabular}{|c|c|c|c|c|}
\hline Well & Location & $\begin{array}{c}\text { Rate } \\
\left(\mathbf{f t}^{3} / \mathbf{d}\right)\end{array}$ & $\begin{array}{c}\text { Duration } \\
\text { (d) }\end{array}$ & $\begin{array}{l}\text { Start Time } \\
\text { (d) }\end{array}$ \\
\hline DNAPL Injector & $(10,5,1)$ & 0.02 & 1.0 & 0.0 \\
\hline Water Injector & $(1,5,1-10)$ & 2.0 & 1.0 & 1.0 \\
\hline Water Extractor & $(20,5,1-10)$ & 2.0 & 1.0 & 1.0 \\
\hline Natural Gradient & $2 \mathrm{E}-04$ & & & \\
\hline NAPL Injected & $0.02 \mathrm{ft}^{3}$ & & & \\
\hline NAPL Produced & $0.00 \mathrm{ft}^{3}$ & & & \\
\hline NAPL in Volume & $0.02 \mathrm{ft}^{3}$ & & & \\
\hline $\begin{array}{ll}\text { Total } & \text { Pore } \\
\text { Volume } & \end{array}$ & $2.0 \mathrm{ft}^{3}$ & & & \\
\hline $\begin{array}{l}\text { Average NAPL } \\
\text { Saturation }\end{array}$ & 0.01 & & & \\
\hline
\end{tabular}

Table 6.4: Summary of Tracers and Relevant Properties

\begin{tabular}{|ccc|}
\hline & Partition Coefficient & Diffusion Coefficient \\
Tracer & between water/TCE & in water \\
& $(\mathrm{mg} / \mathrm{L}) /(\mathrm{mg} / \mathrm{L})$ & $\left(\mathrm{cm}^{2} / \mathrm{s}\right)$ \\
\hline 1-propanol & 0.0 & $1.02 \times 10^{-5}$ \\
2,3-dimethyl-3-pentanol & 29.0 & $6.8 \times 10^{-6}$ \\
1-heptanol & 94.4 & $7.1 \times 10^{-6}$ \\
\hline
\end{tabular}

Table 6.5: Reported Values for Tortuosity in Various Consolidated Porous Media

\begin{tabular}{|l|l|c|c|}
\hline Source & Porous Medium & Porosity & Tortuosity \\
\hline \hline Donaldson et al., 1976 & Bandera sandstone & 0.18 & 0.29 \\
\hline & Berea sandstone & 0.19 & 0.41 \\
\hline Neretnieks, 1993 & granite & $0.006-$ & $0.002-$ \\
& & 0.0007 & 0.0009 \\
\hline & gneiss & $0.004-$ & $0.002-$ \\
& & 0.0006 & 0.0001 \\
\hline
\end{tabular}


Table 6.6: Comparison of NAPL Saturation and NAPL Volume Estimates for Various Positive MRV Errors

\begin{tabular}{|c|c|c|c|c|}
\hline Condition & & & & $\begin{array}{l}\text { Better } \\
\text { relative } \\
\text { estimate }\end{array}$ \\
\hline$\Delta \bar{V}_{1}>\Delta \bar{V}_{2}$ & $V_{N}^{*}<V_{N}$ & $S_{N}^{*}<S_{N}$ & $\frac{V_{N}}{S_{N}} \frac{S_{N}^{*}}{V_{N}^{*}}<1$ & $V_{N}$ \\
\hline$\Delta \bar{V}_{1}=\Delta \bar{V}_{2}$ & $V_{N}^{*}=V_{N}$ & $S_{N}^{*}<S_{N}$ & $\frac{V_{N}}{S_{N}} \frac{S_{N}^{*}}{V_{N}^{*}}<1$ & $V_{N}$ \\
\hline$\frac{\bar{V}_{1}}{\bar{V}_{2}} \Delta \bar{V}_{2}<\Delta \bar{V}_{1}<\Delta \bar{V}_{2}$ & $V_{N}^{*}>V_{N}$ & $S_{N}^{*}<S_{N}$ & $\frac{V_{N}}{S_{N}} \frac{S_{N}^{*}}{V_{N}^{*}}<1$ & indeterminate \\
\hline$\Delta \bar{V}_{1}=\frac{\bar{V}_{1}}{\bar{V}_{2}} \Delta \bar{V}_{2}$ & $V_{N}^{*}>V_{N}$ & $S_{N}^{*}=S_{N}$ & $\frac{V_{N}}{S_{N}} \frac{S_{N}^{*}}{V_{N}^{*}}<1$ & $S_{N}$ \\
\hline$\Delta \bar{V}_{1}<\frac{\bar{V}_{1}}{\bar{V}_{2}} \Delta \bar{V}_{2}$ & $V_{N}^{*}>V_{N}$ & $S_{N}^{*}>S_{N}$ & $\frac{V_{N}}{S_{N}} \frac{S_{N}^{*}}{V_{N}^{*}}<1$ & $S_{N}$ \\
\hline
\end{tabular}




\subsection{Figures}

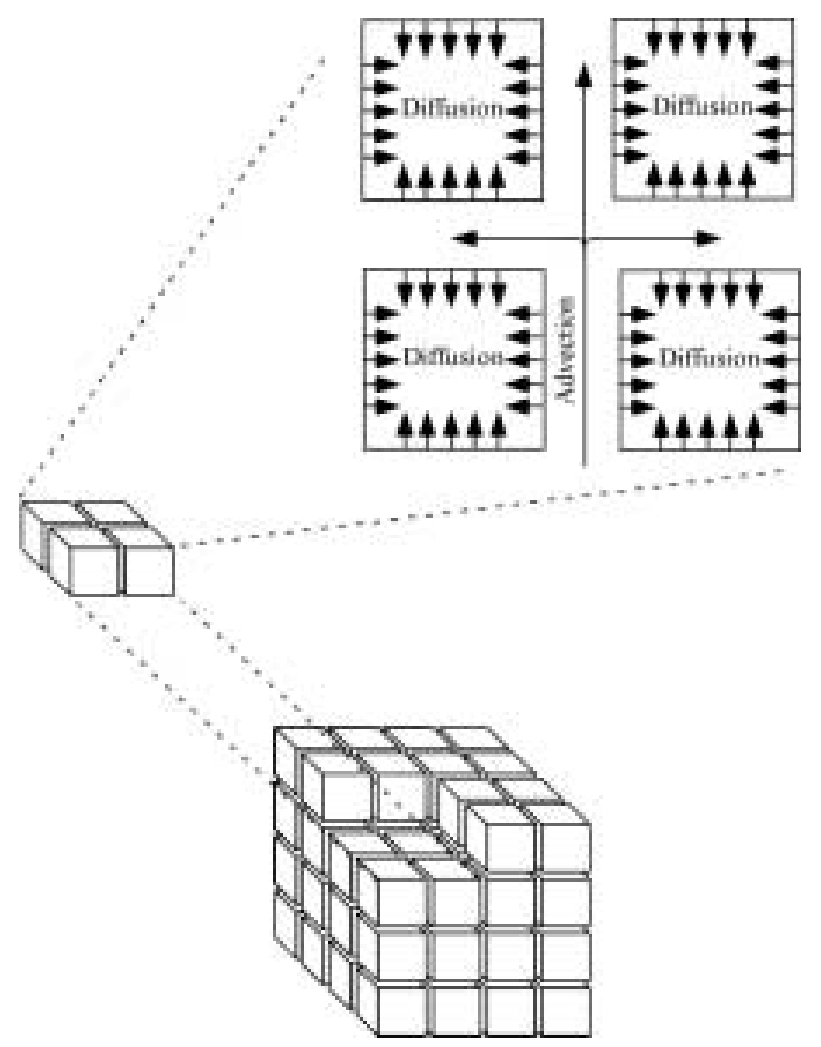

Figure 6.1: Sugarcube dual porosity model.

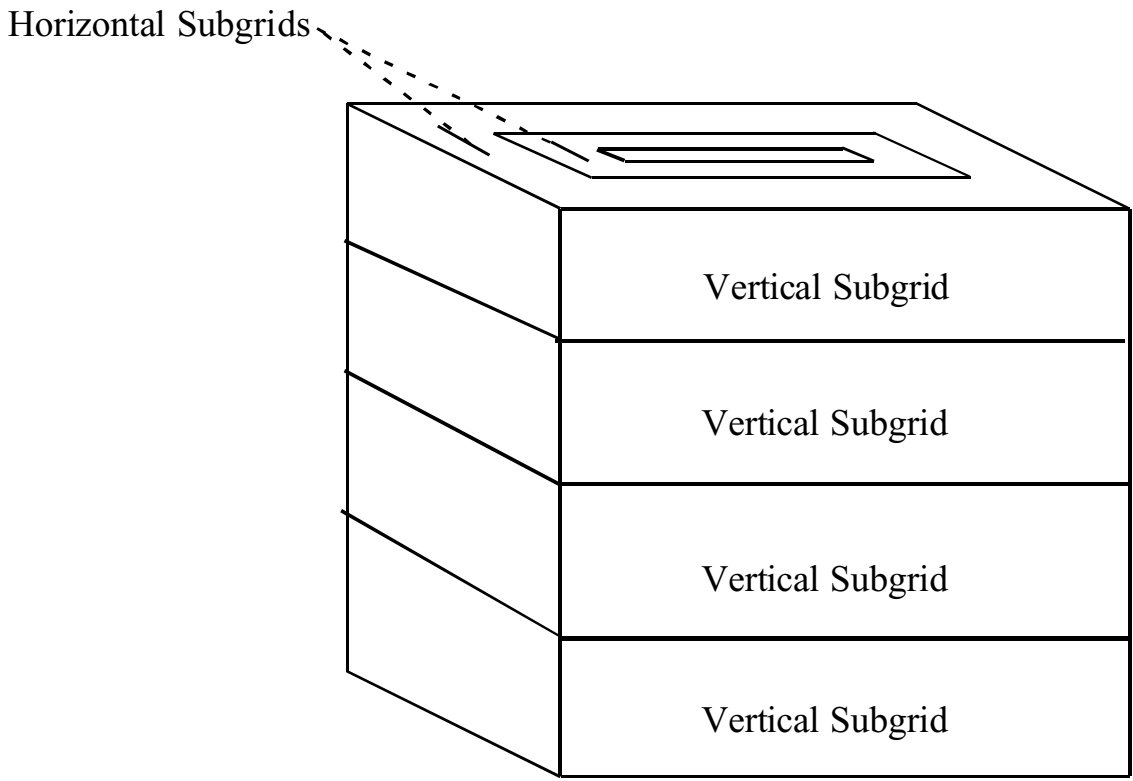

Figure 6.2: Matrix block subgridding in UTCHEM. 

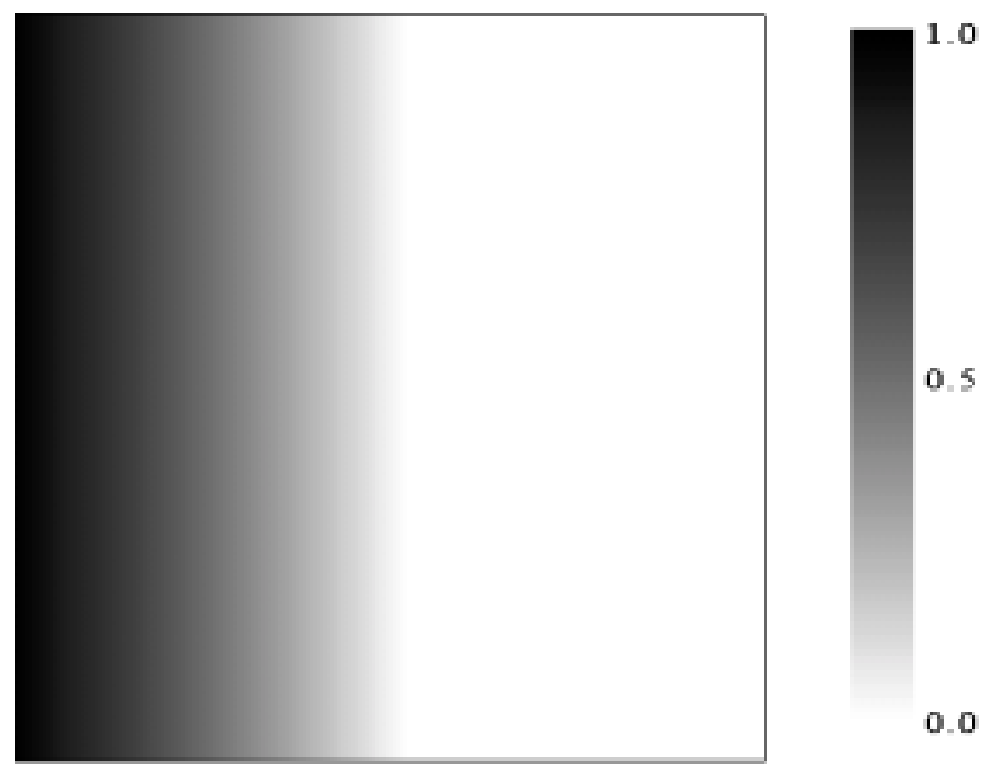

Increasing distance into matrix block --.>

Figure 6.3: Illustration of concentration distribution in matrix block.
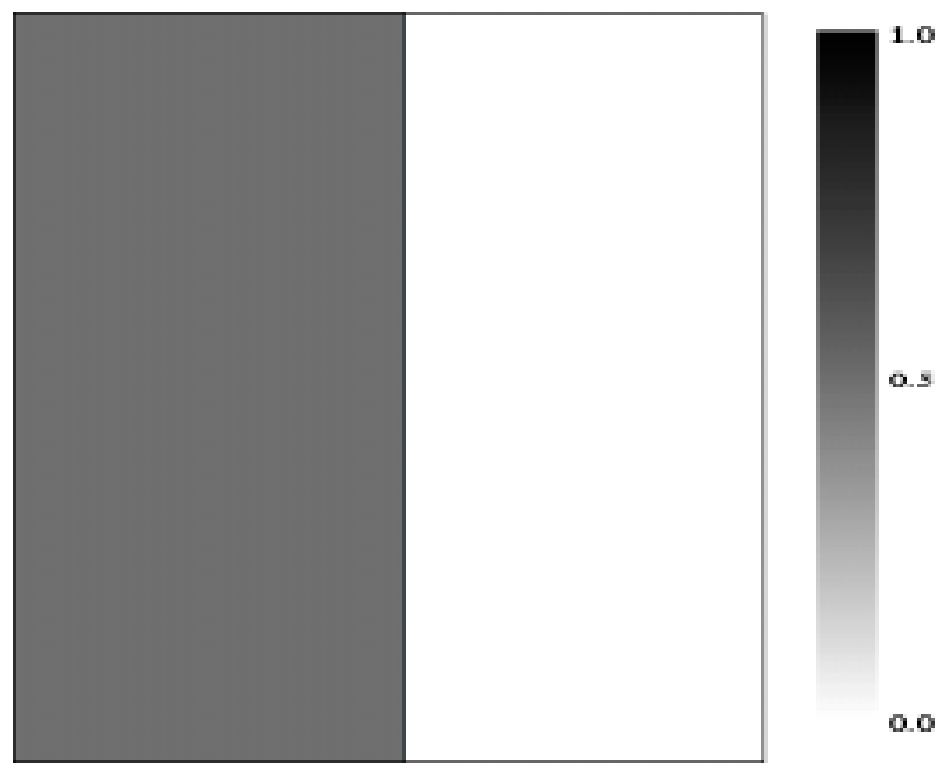

Increasing distance into matrix block --->

Figure 6.4: Illustration of concentration distribution, 2 equal subgrids. 

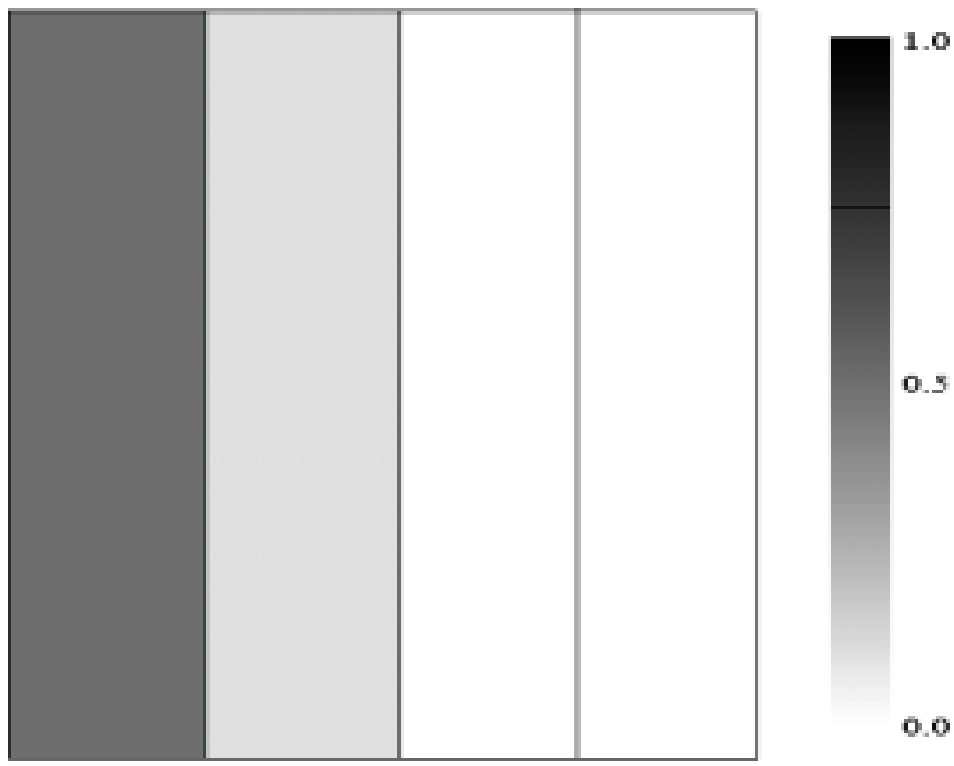

Increasing distance into matrix block -..>

Figure 6.5: Illustration of concentration distribution, 4 equal subgrids.
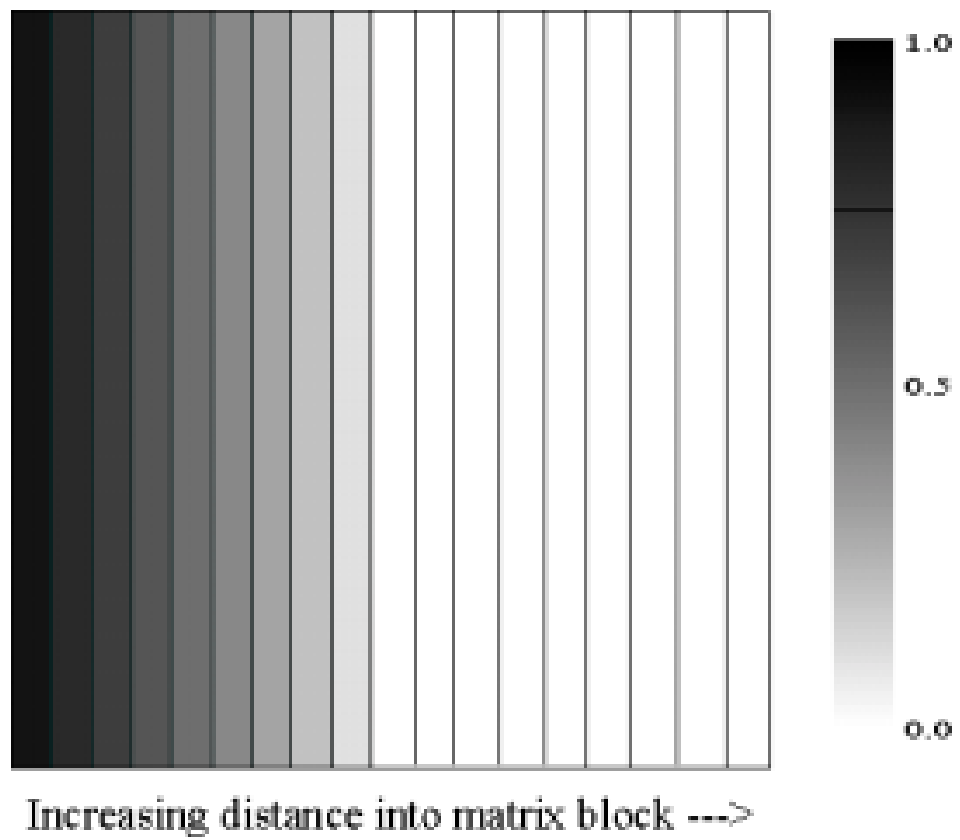

Figure 6.6: Illustration of concentration distribution, 20 equal subgrids. 

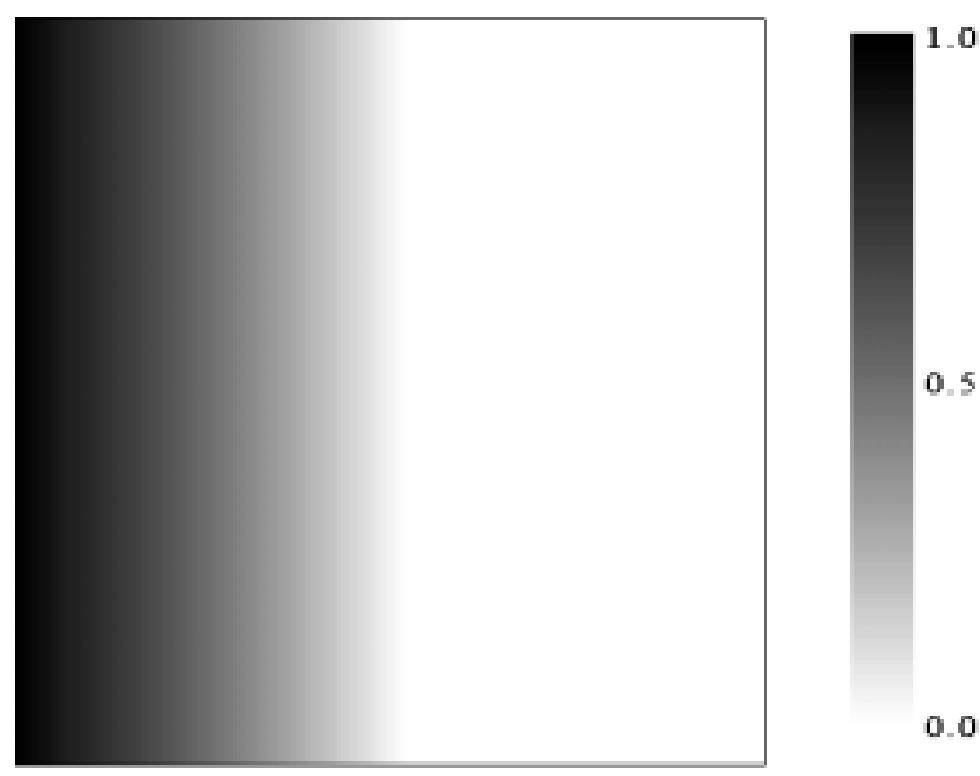

Increasing distance into matrix block -..>
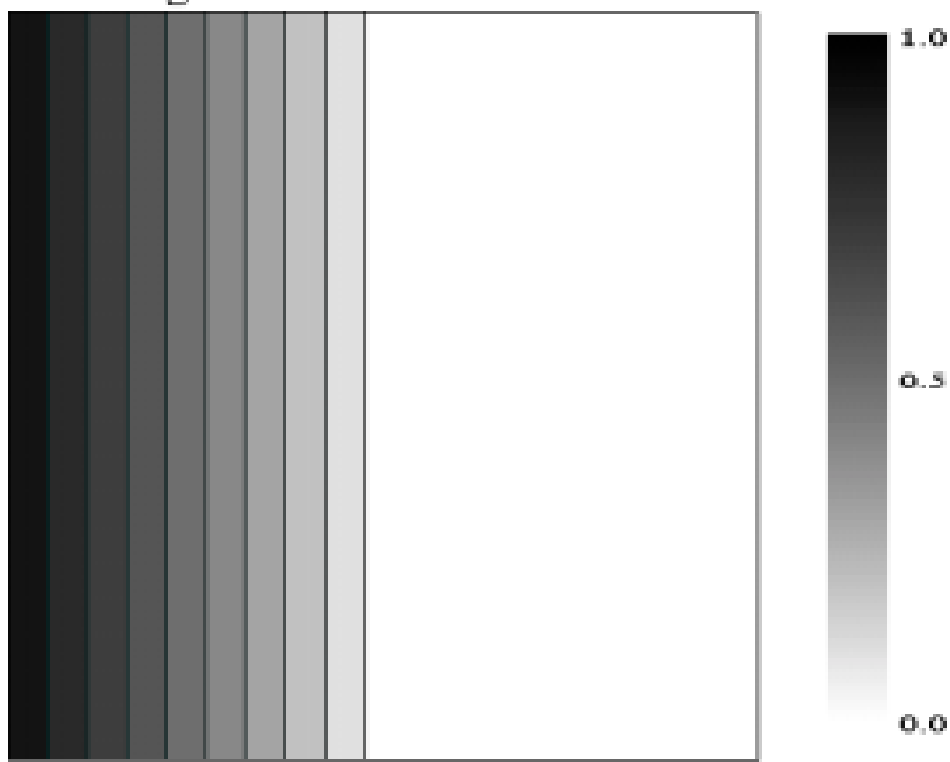

Increasing distance into matrix block ...>

Figure 6.7: Illustrative comparison of 10 unequal subgrid representation of matrix concentrations (bottom) with actual matrix concentrations (top). 


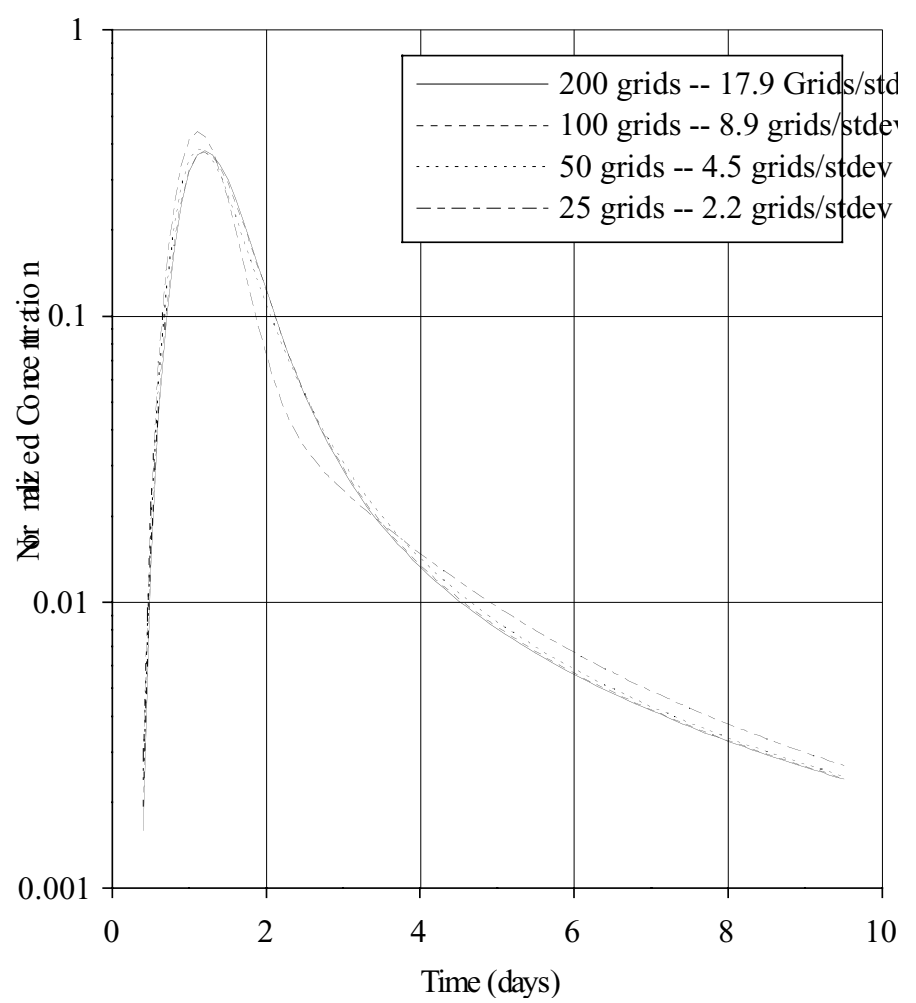

Figure 6.8: Conservative tracer response curves at varying vertical subgrid resolutions.

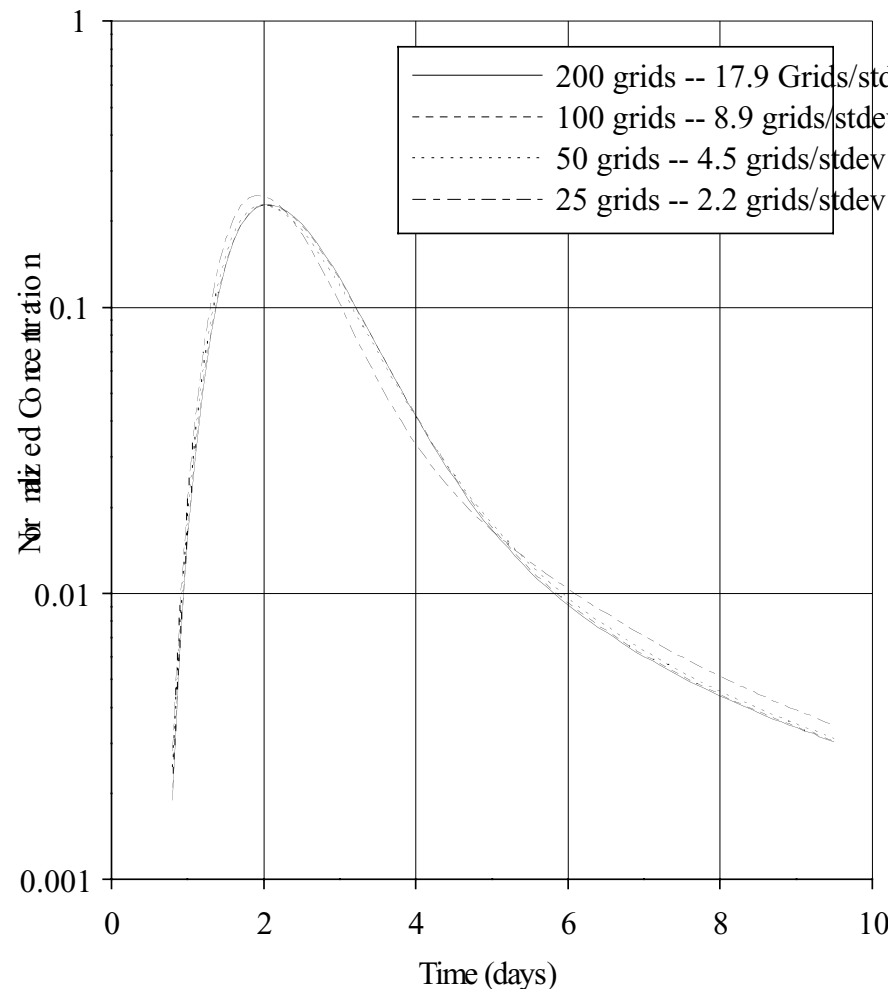

Figure 6.9: Partitioning tracer response curves for varying lengths of vertical subgrid refinement. 


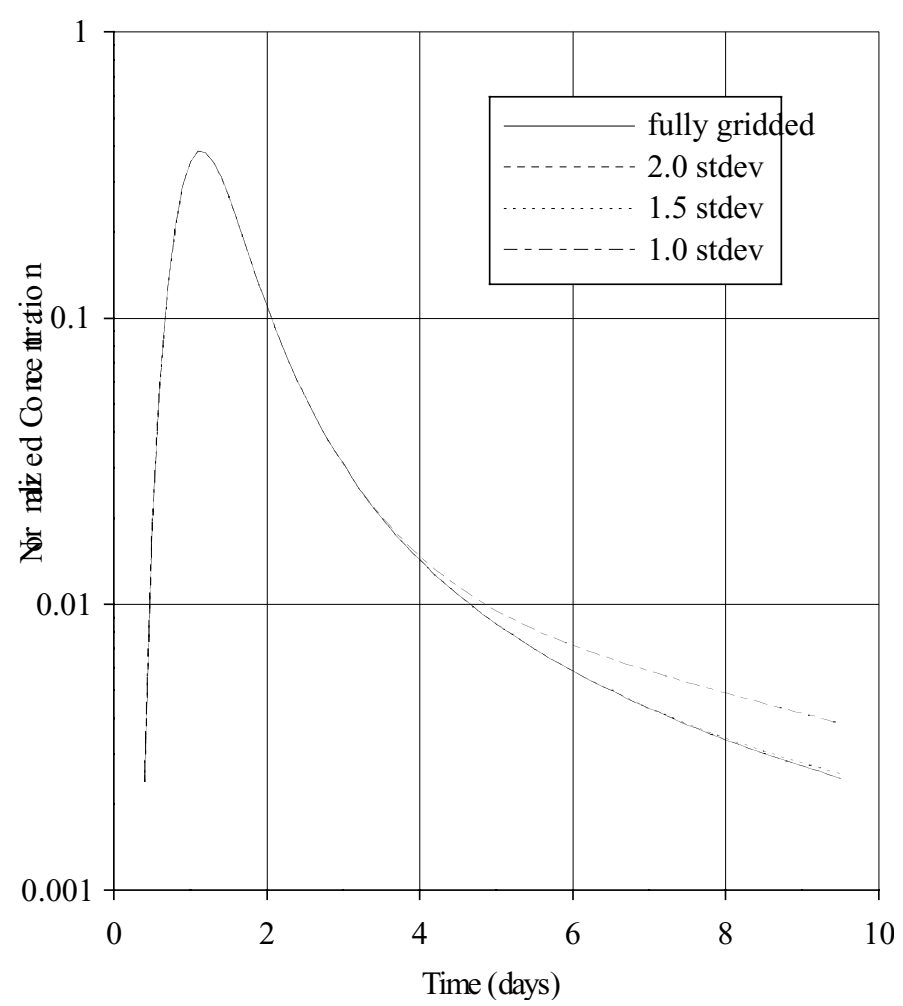

Figure 6.10: Conservative tracer response curves for varying lengths of vertical subgrid refinement.

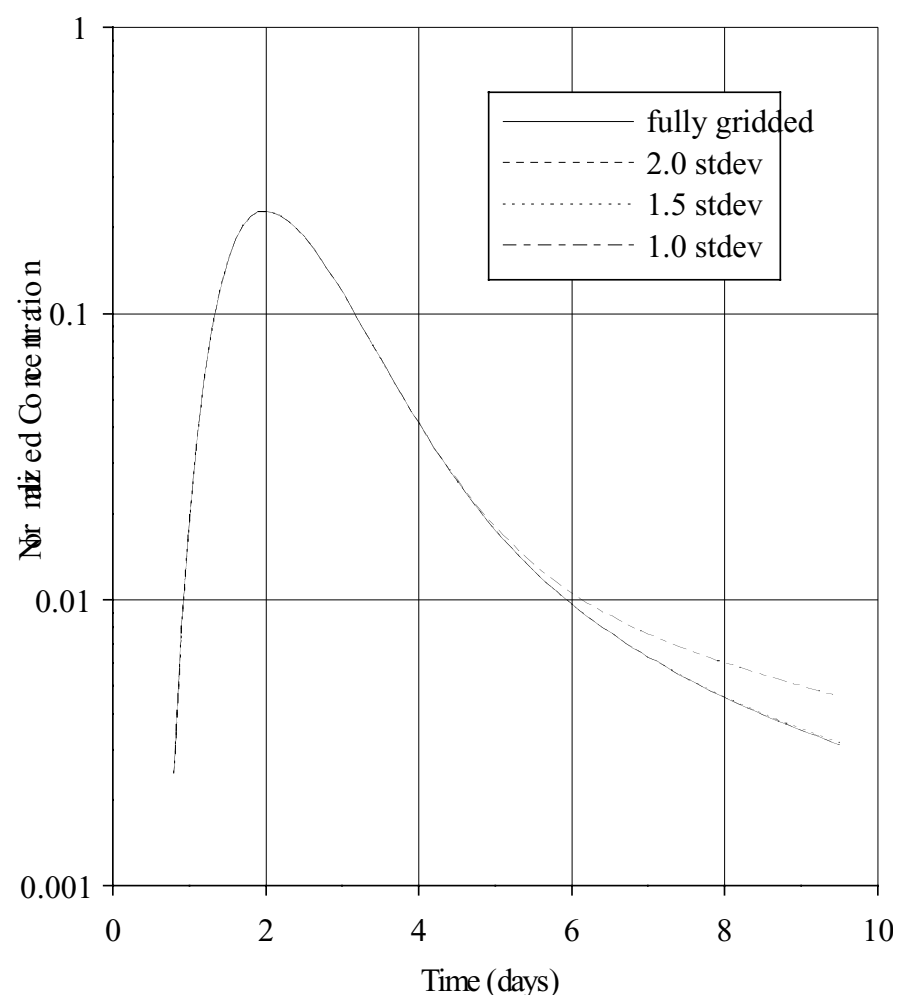

Figure 6.11: Partitioning tracer response curves for refined subgrids at varying vertical distances from the matrix block edge. 


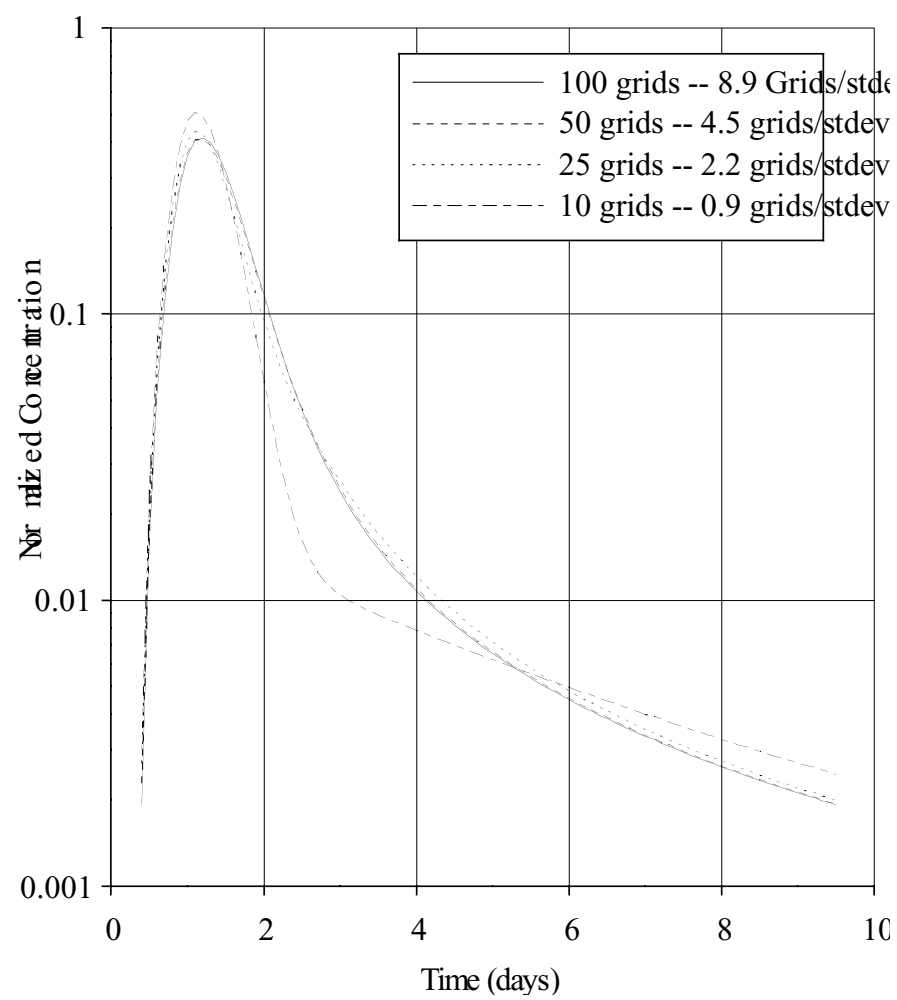

Figure 6.12: Conservative tracer response curves at varying horizontal subgrid resolutions.

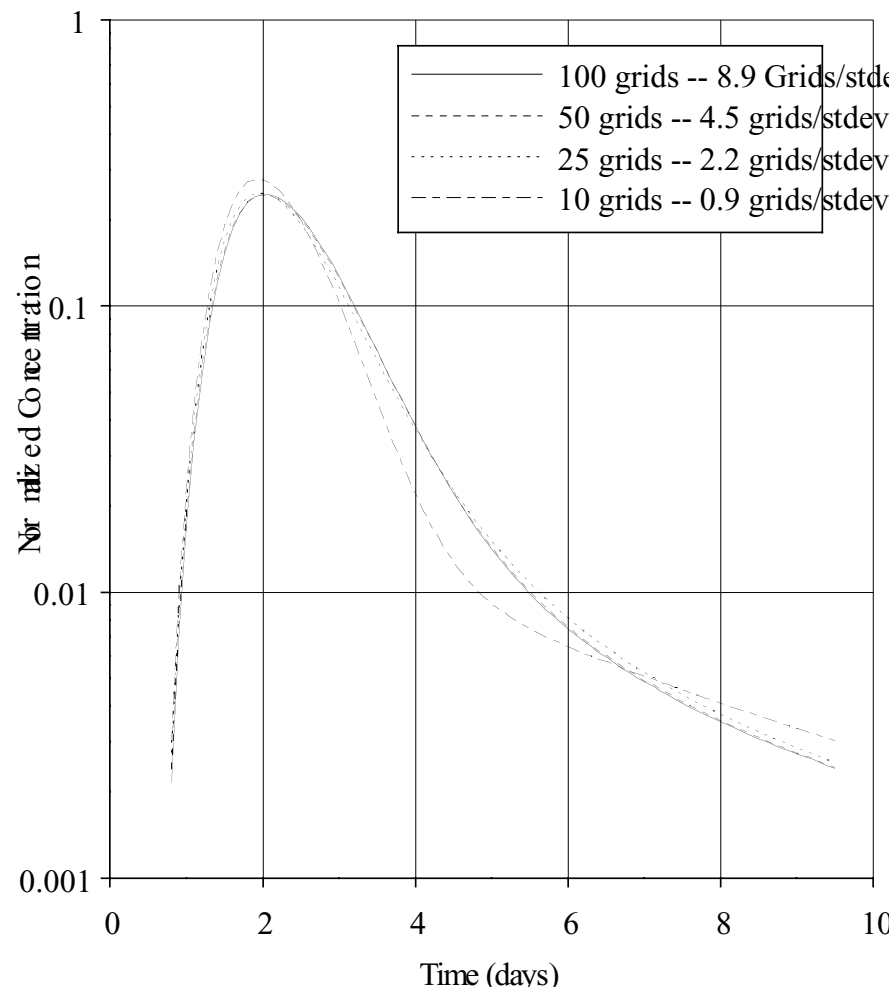

Figure 6.13: Partitioning tracer response curves at varying horizontal subgrid resolutions. 


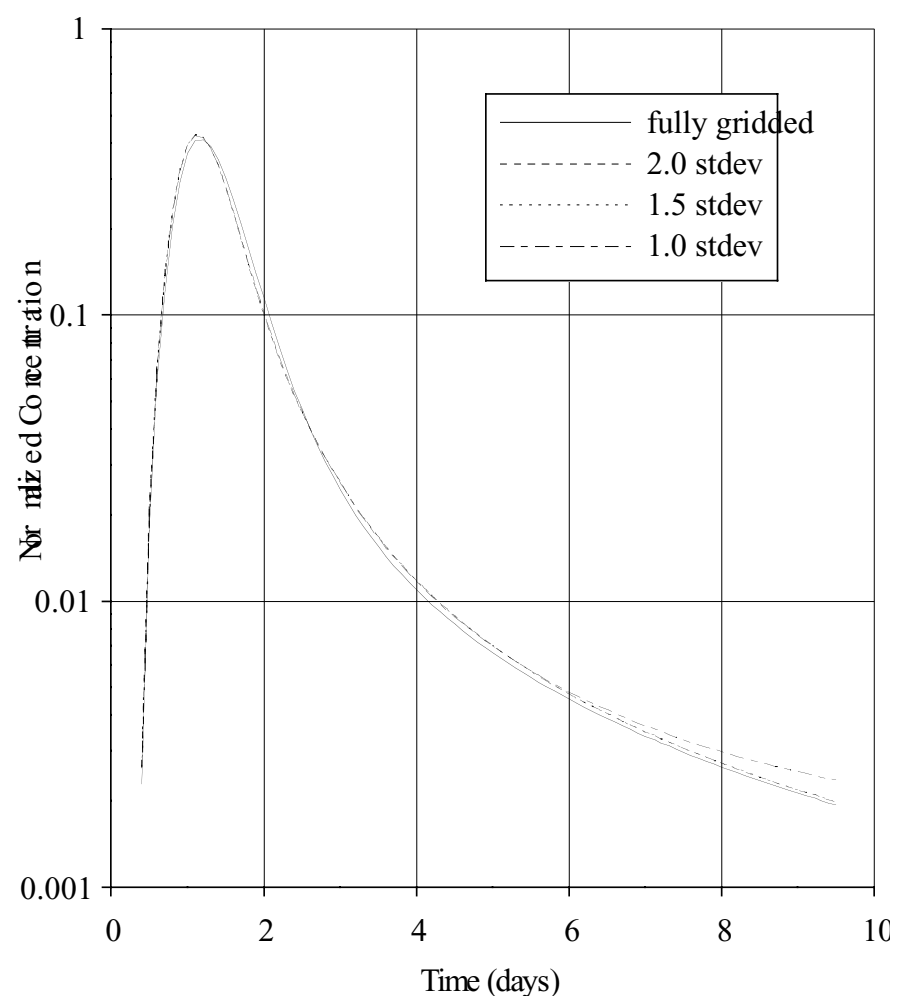

Figure 6.14: Conservative tracer response curves for varying lengths of horizontal subgrid refinement.

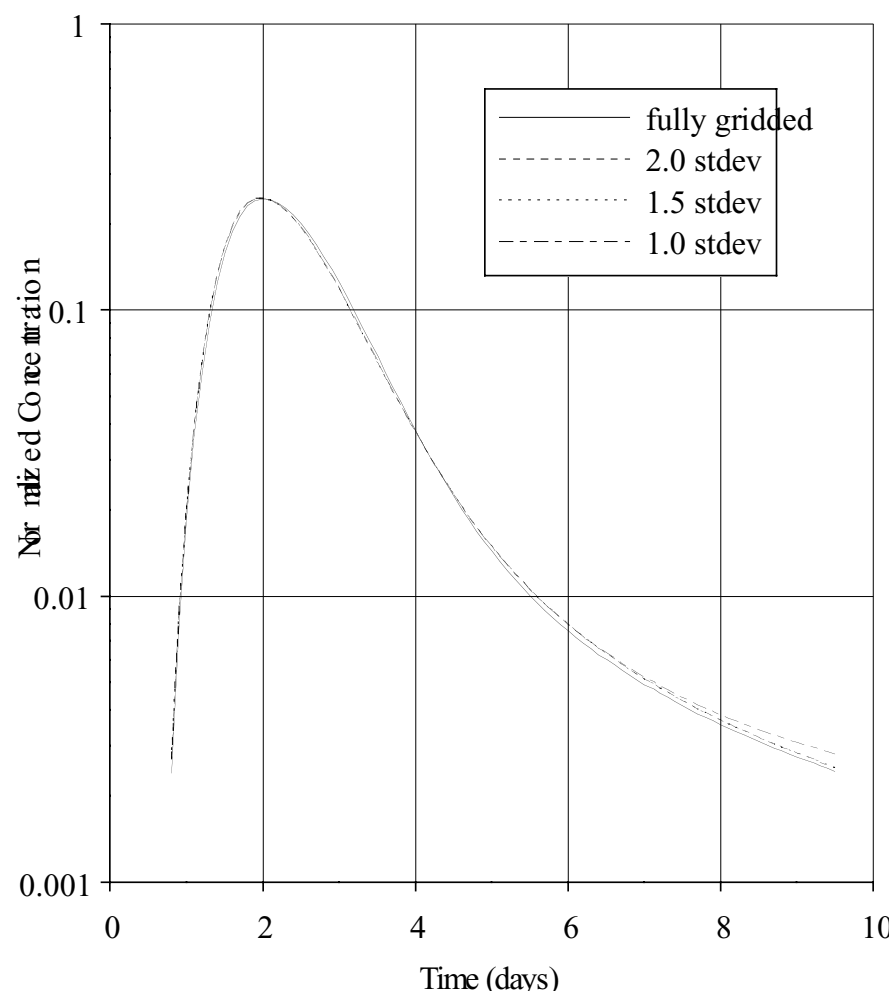

Figure 6.15 Partitioning tracer response curves for varying lengths of horizontal subgrid refinement. 


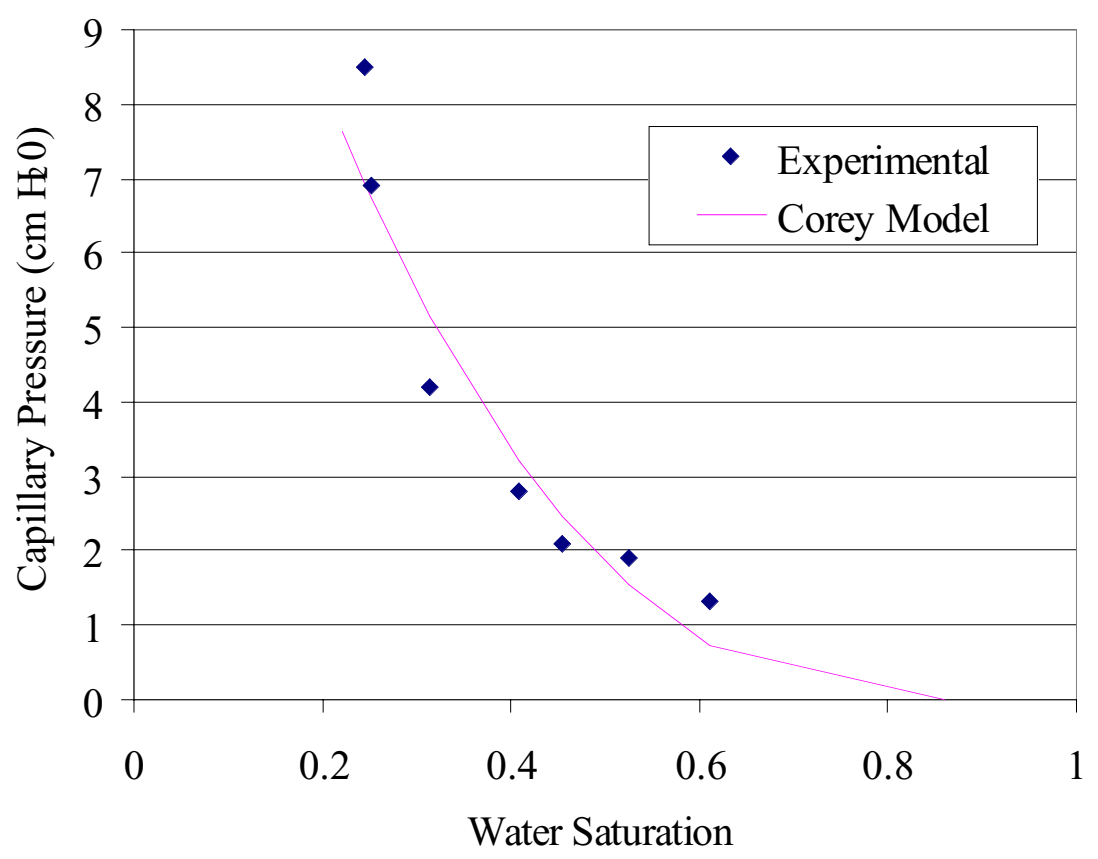

Figure 6.16: Comparison of Corey imbibition model with Reitsma and Kueper (1994) experimental data.

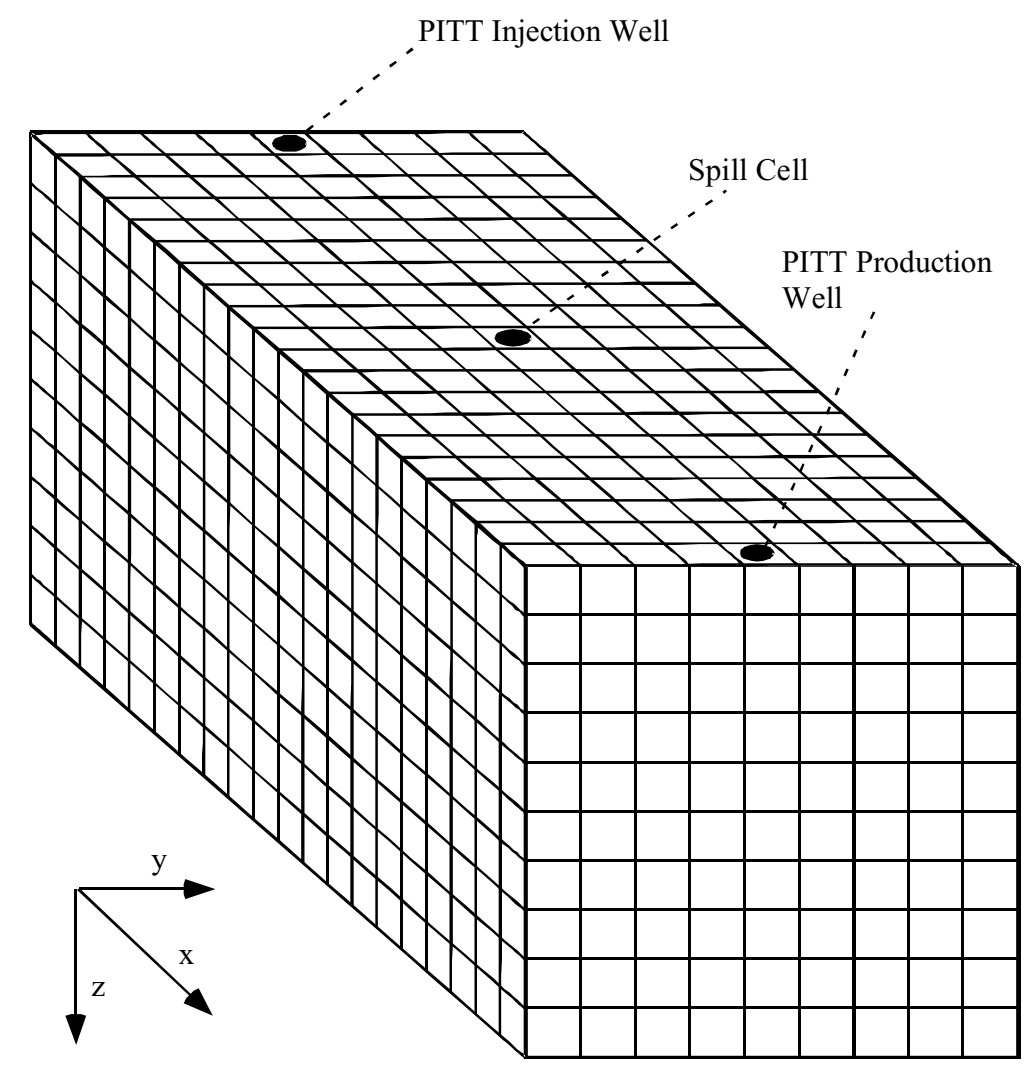

Figure 6.17: xyz grid for DNAPL spill and PITT simulations. 


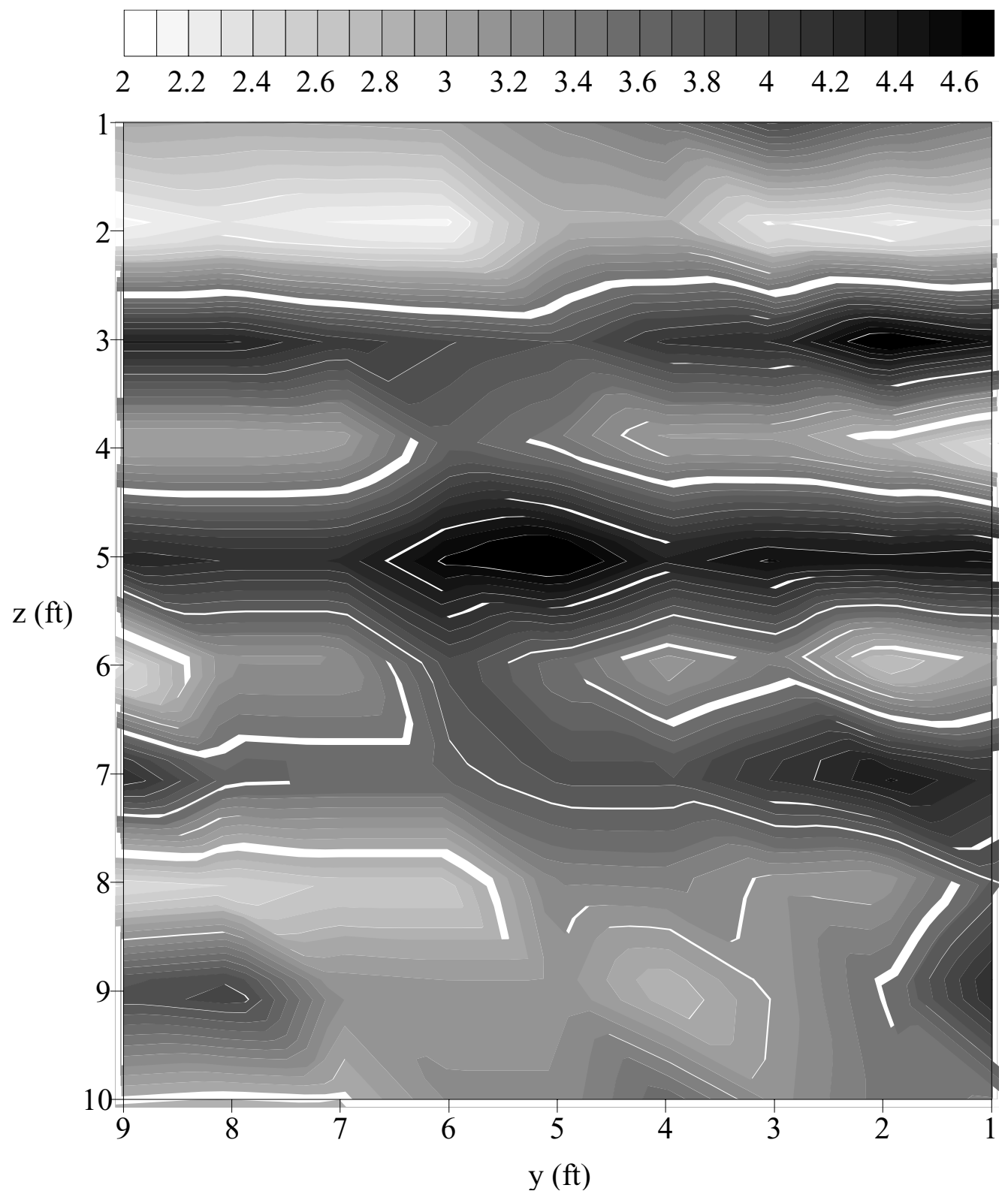

Figure 6.18: $\mathrm{z}-\mathrm{y}$ slice of $\log (\mathrm{k})(\log ($ Darcies $))$ distribution, $\mathrm{x}=10 \mathrm{ft}$. 


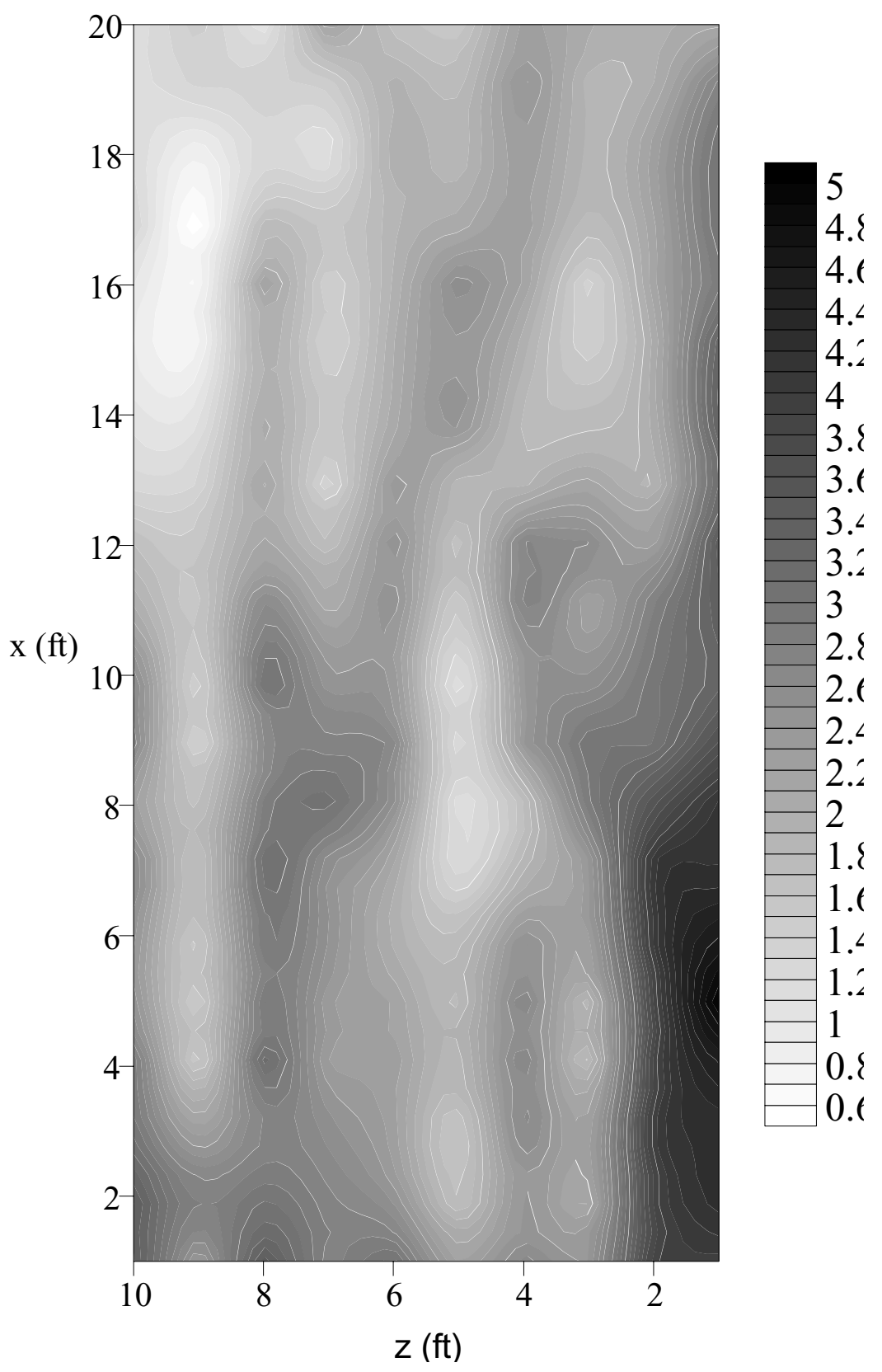

Figure 6.19: $\mathrm{x}-\mathrm{z}$ slice of $\log (\mathrm{k})(\log ($ Darcies $))$ distribution, $\mathrm{y}=5 \mathrm{ft}$. 


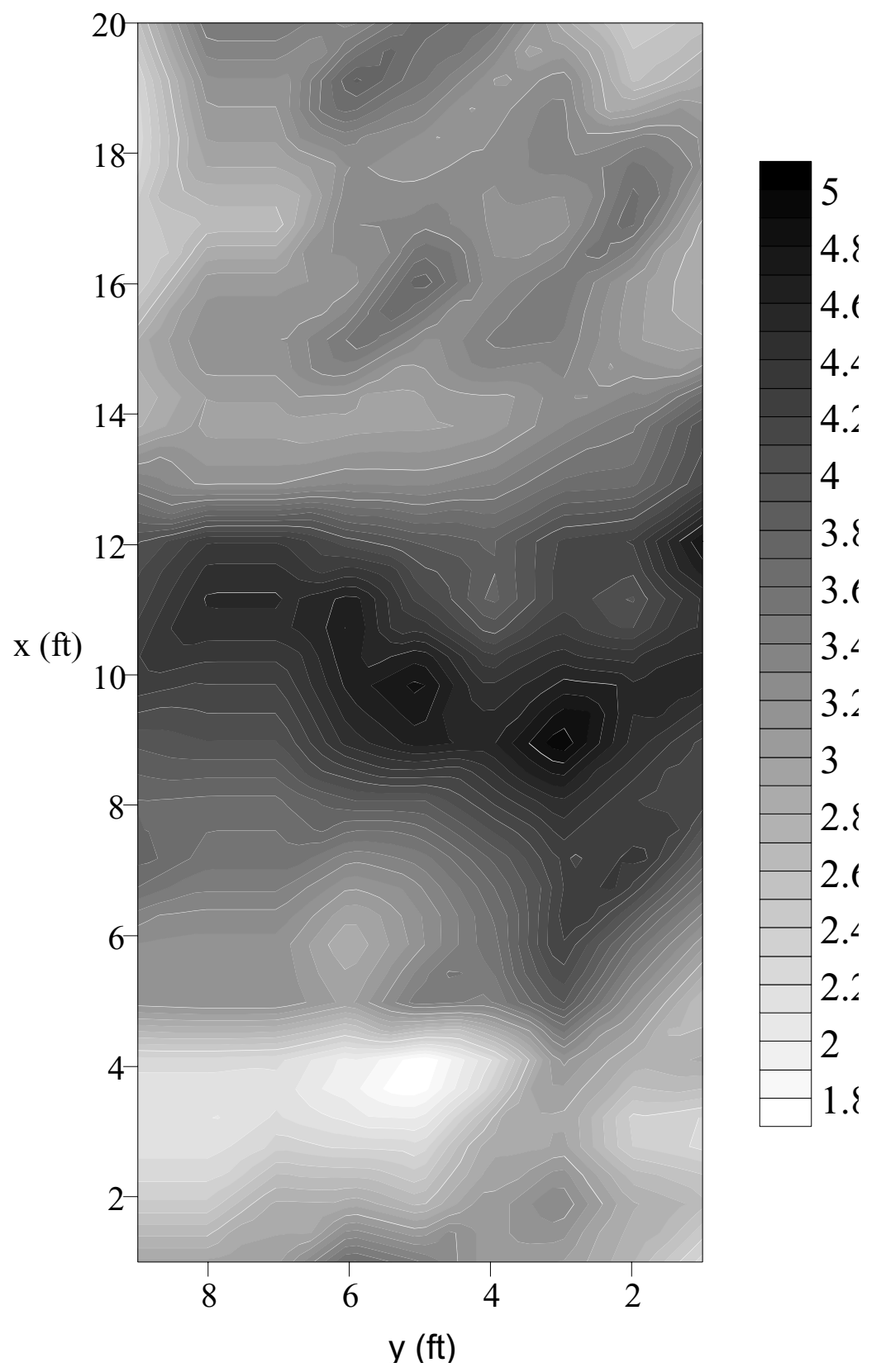

Figure 6.20: $\mathrm{x}-\mathrm{y}$ slice of $\log (\mathrm{k})(\log ($ Darcies $))$ distribution, $\mathrm{z}=5 \mathrm{ft}$. 


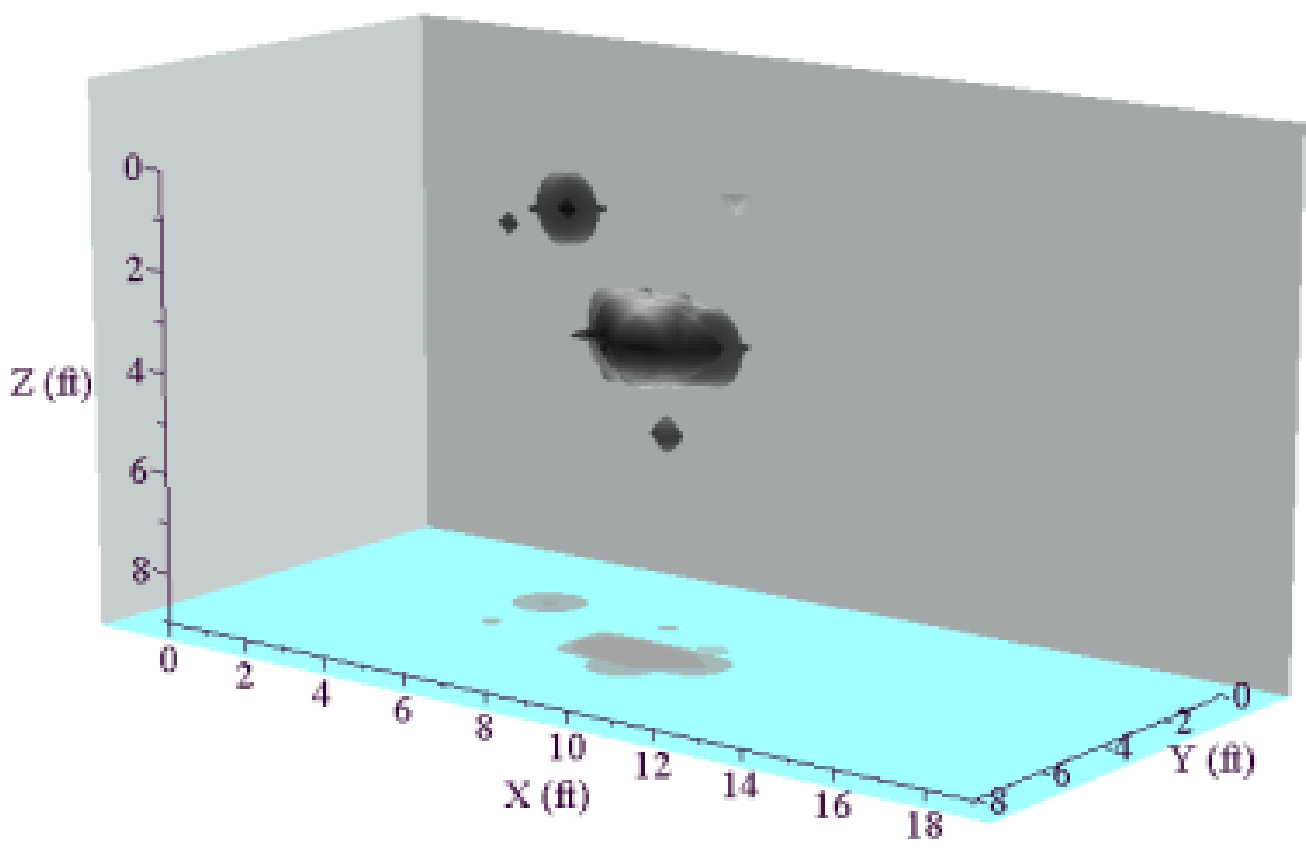

Figure 6.21: $S_{N}=0.01$ isosurface at 0.1 days DNAPL spill.

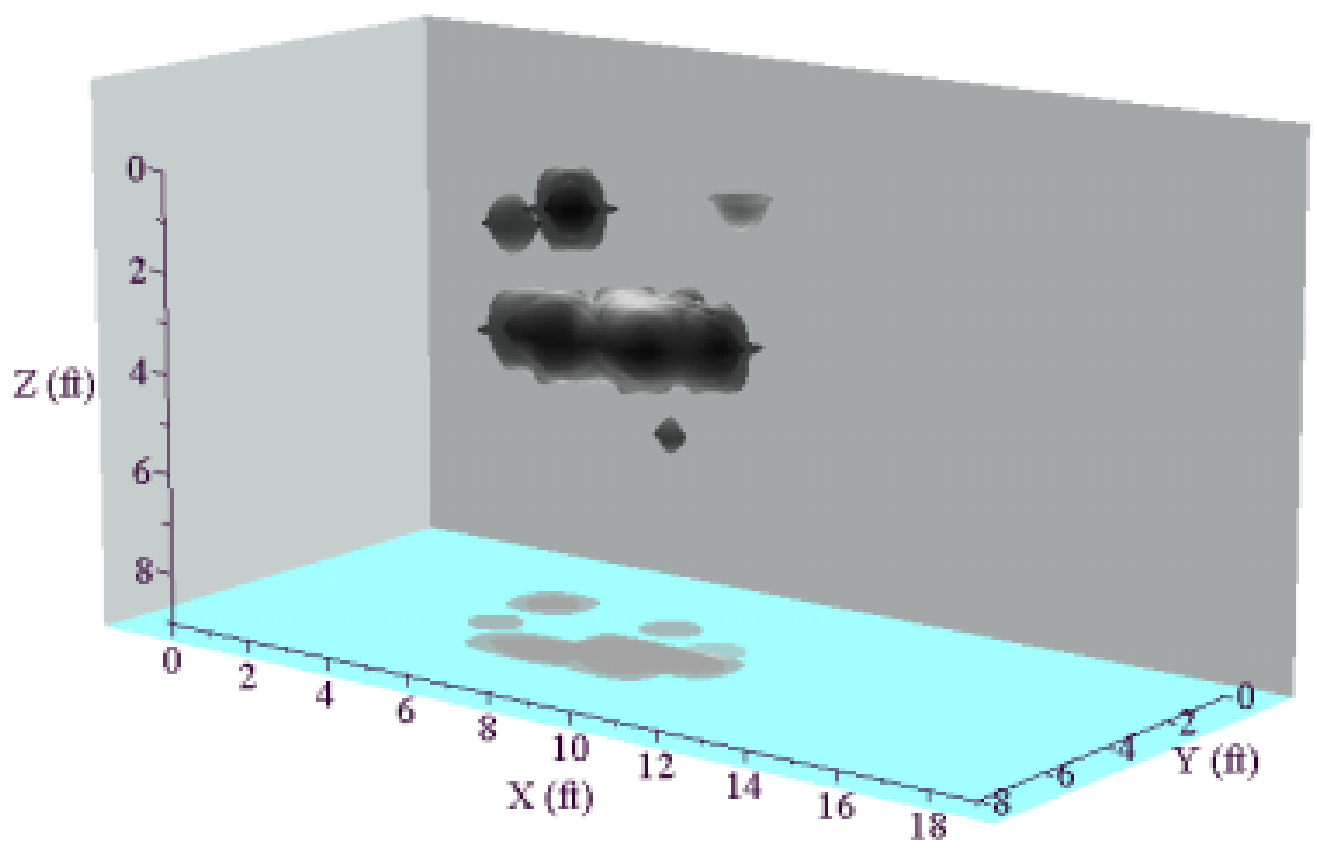

Figure 6.22: $S_{N}=0.01$ isosurface at 0.2 days DNAPL spill. 


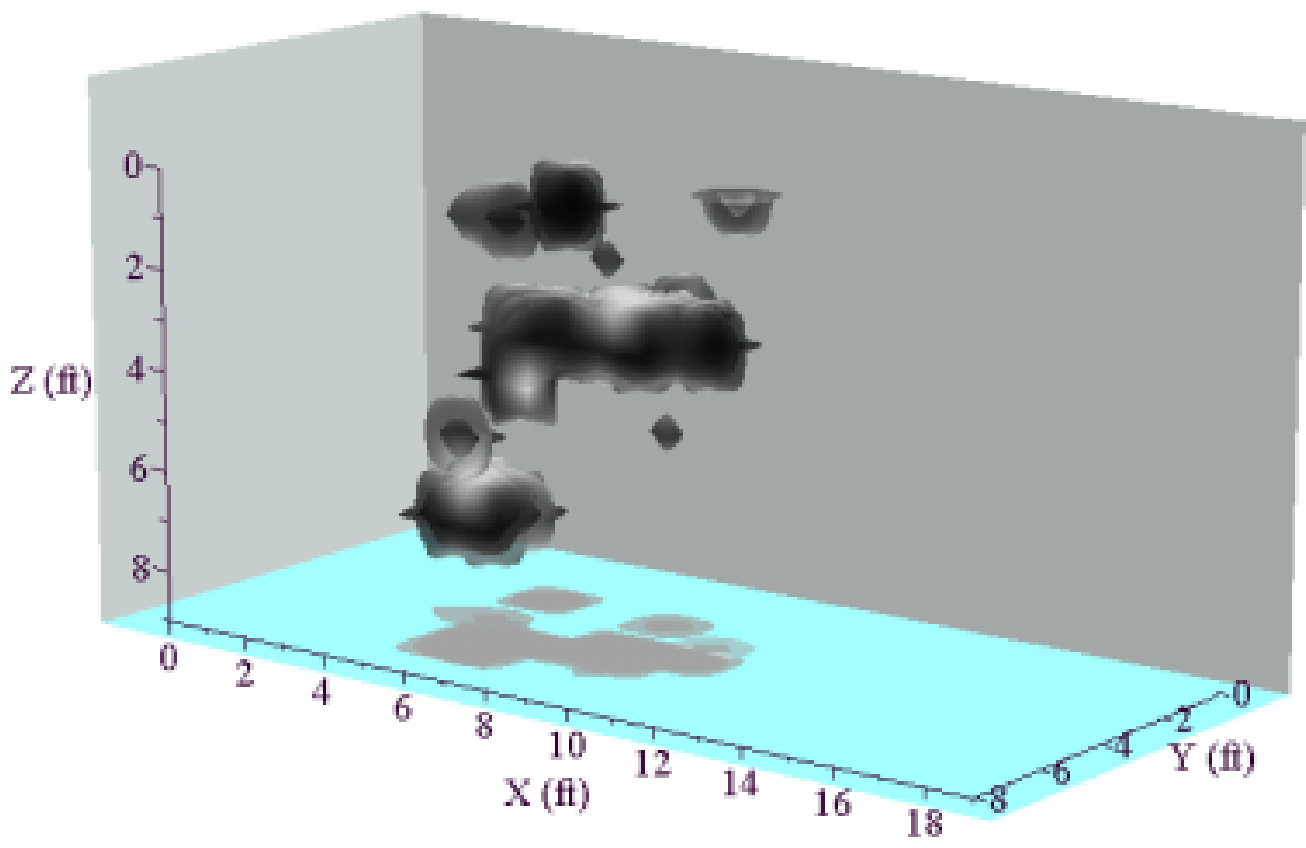

Figure 6.23: $S_{N}=0.01$ isosurface at 0.5 days DNAPL spill.

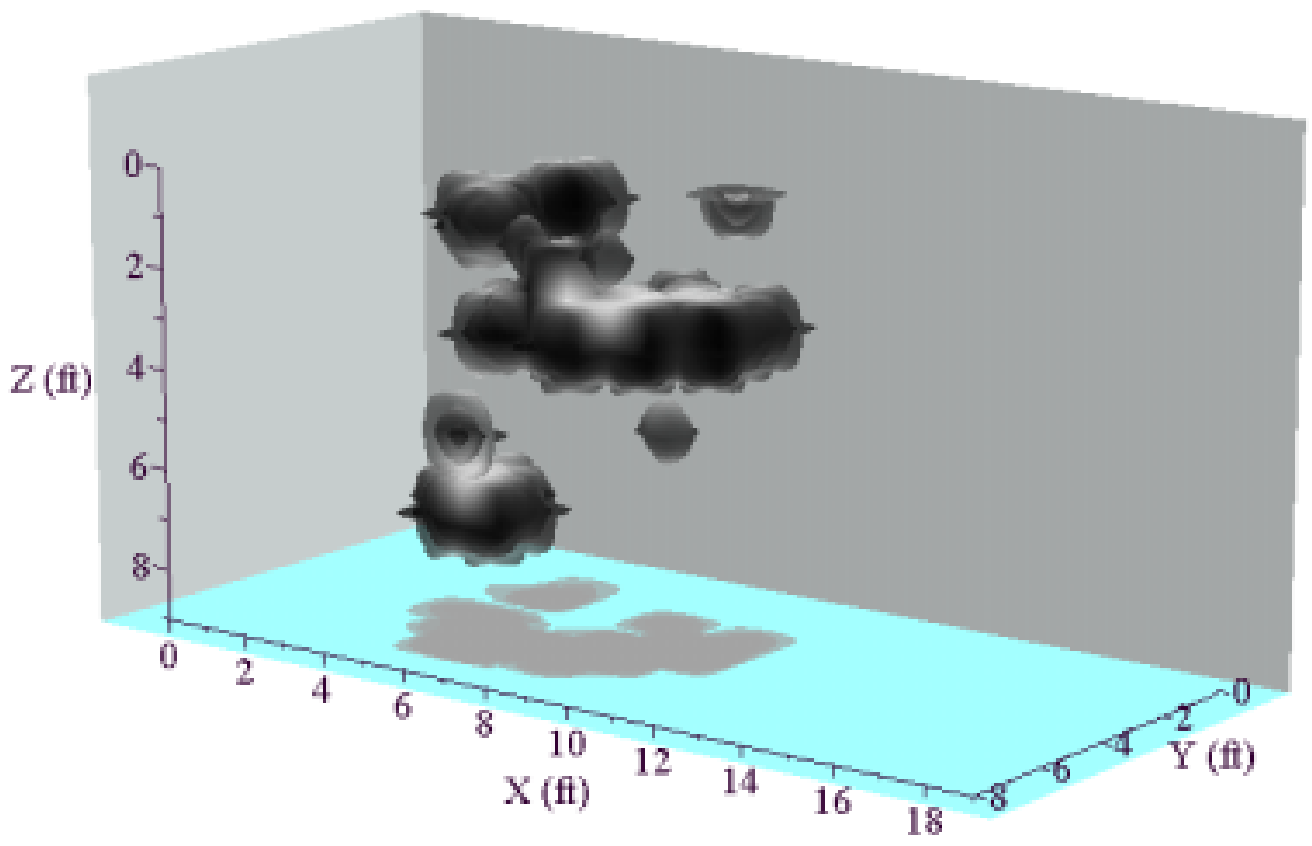

Figure 6.24: $S_{N}=0.01$ isosurface at 1.0 days DNAPL spill. 


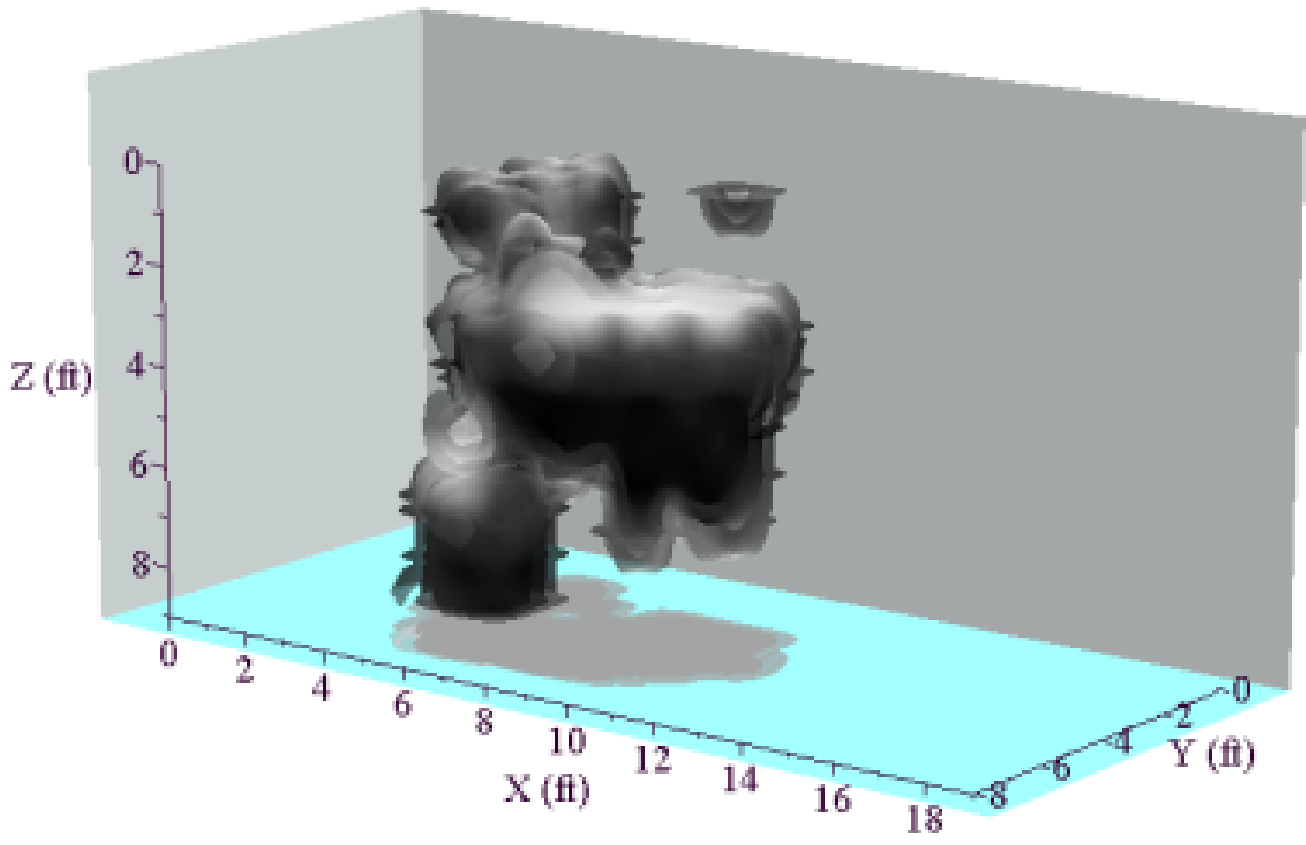

Figure 6.25: $S_{N}=0.01$ isosurface at 0.1 days waterflooding, 1.1 days total.

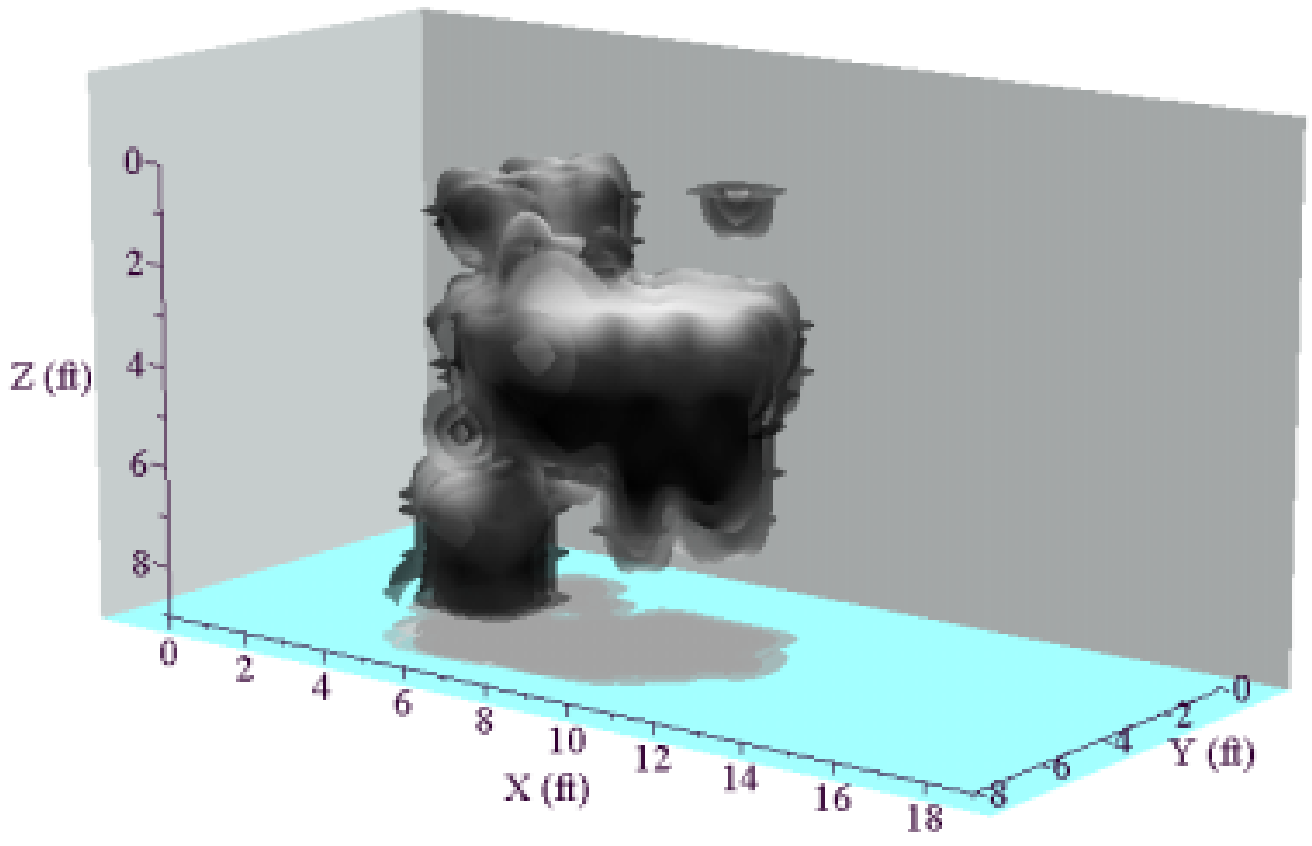

Figure 6.26: $S_{N}=0.01$ isosurface at 1.0 days of waterflooding, 2.0 days total. 

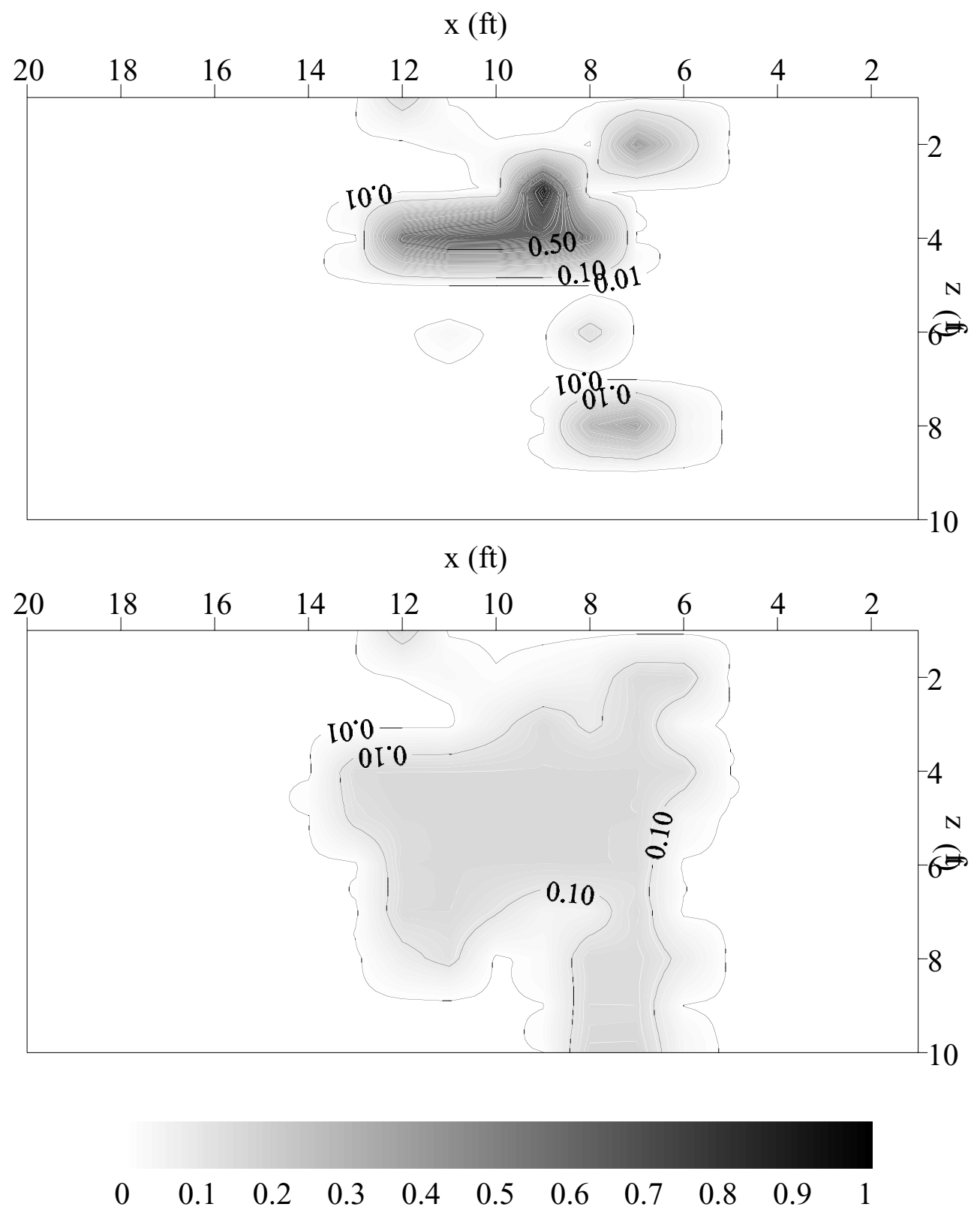

Figure 6.27: Comparison of x-z profile of saturation distribution before (top graph) and after (bottom graph) 1.0 day waterflood. 


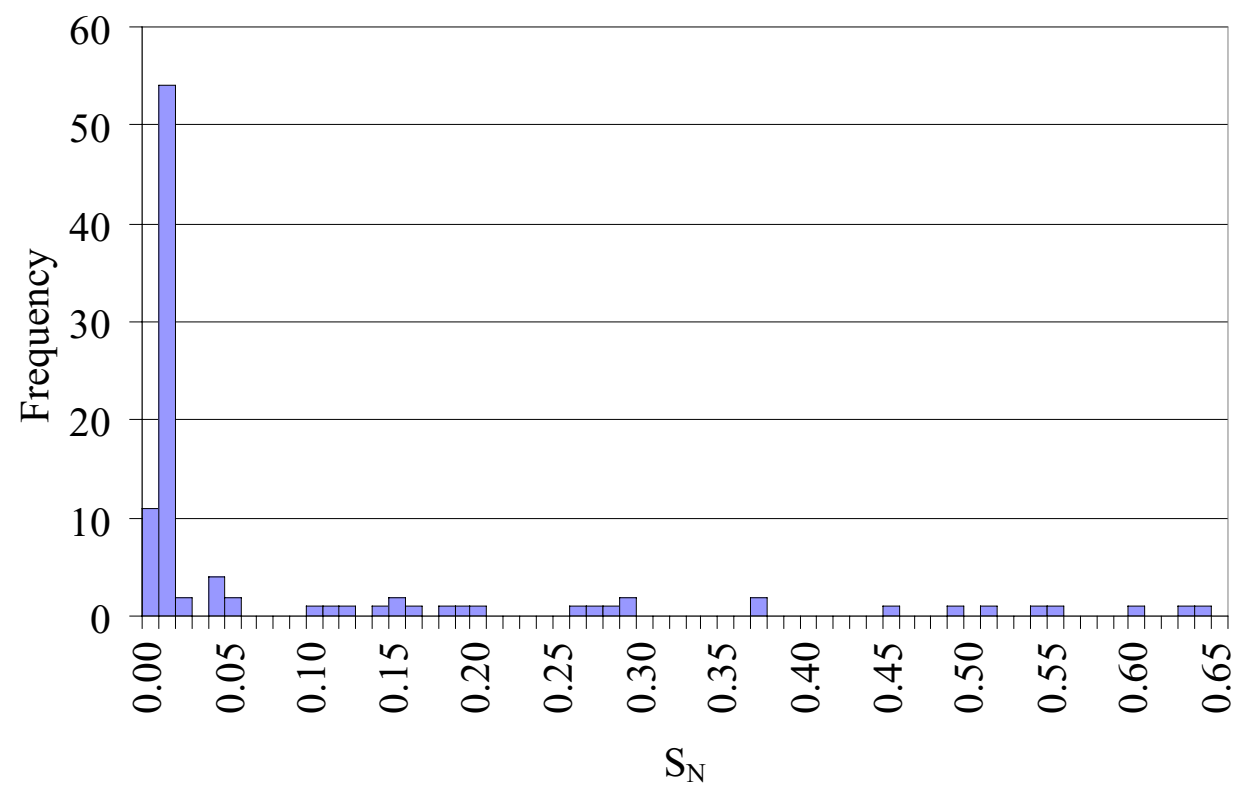

Figure 6.28: Histogram of saturations before waterflood.

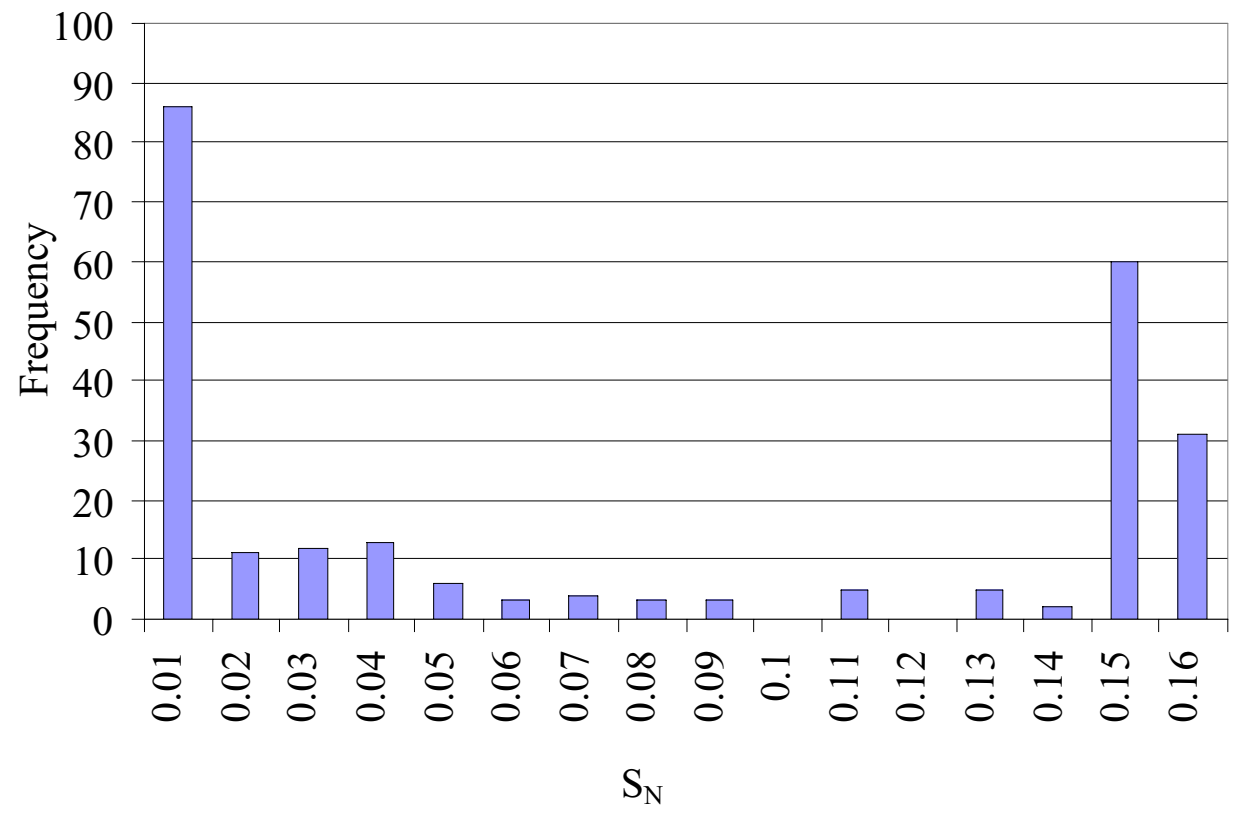

Figure 6.29: Histogram of saturations after 1.0 day waterflood. 


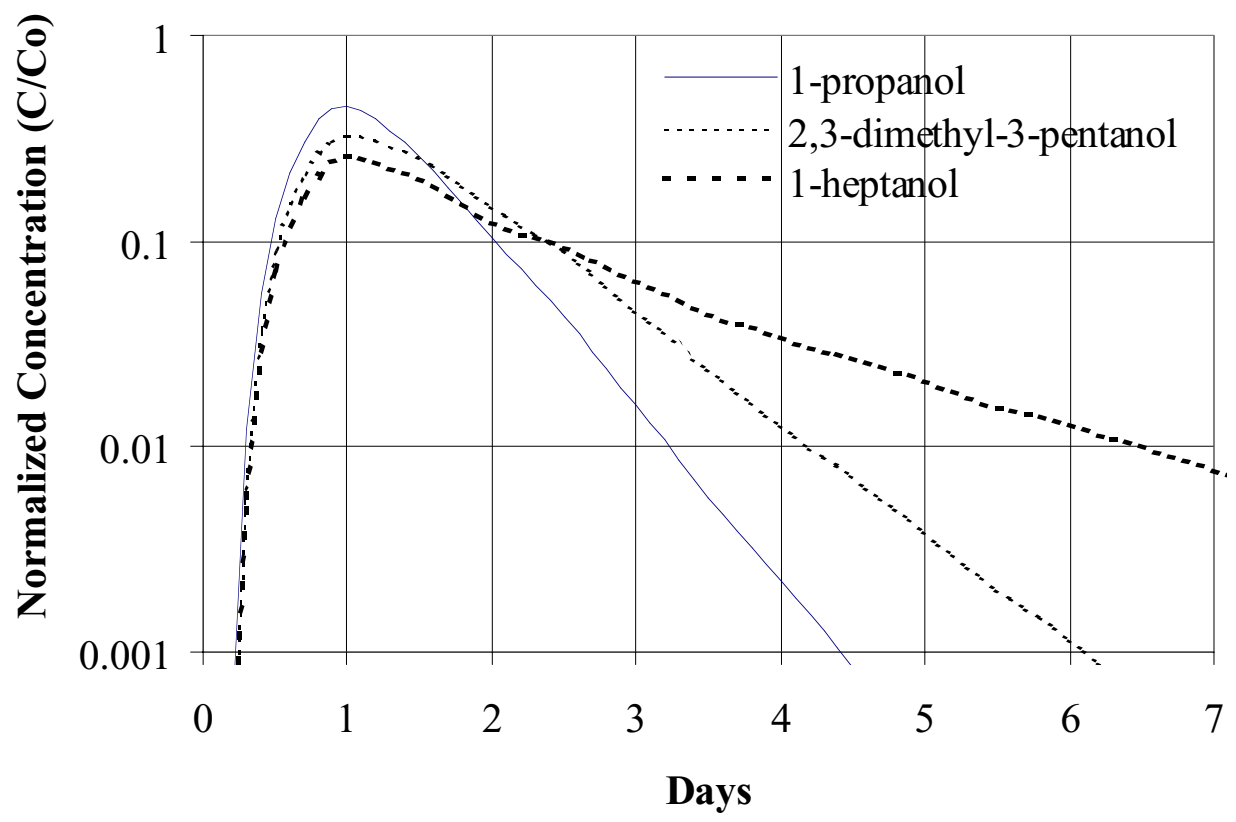

Figure 6.30: Tracer response curves for TT035.

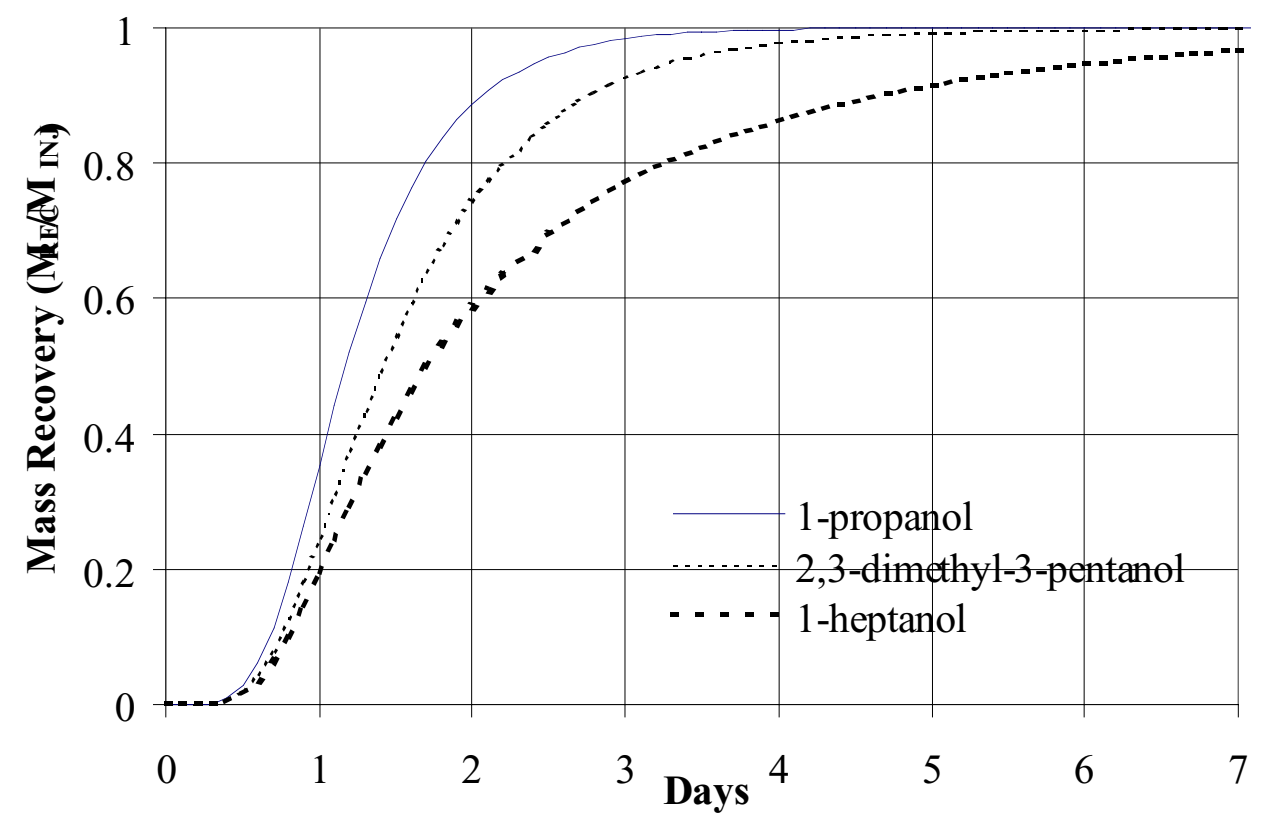

Figure 6.31: Cumulative tracer mass recovery for TT035. 


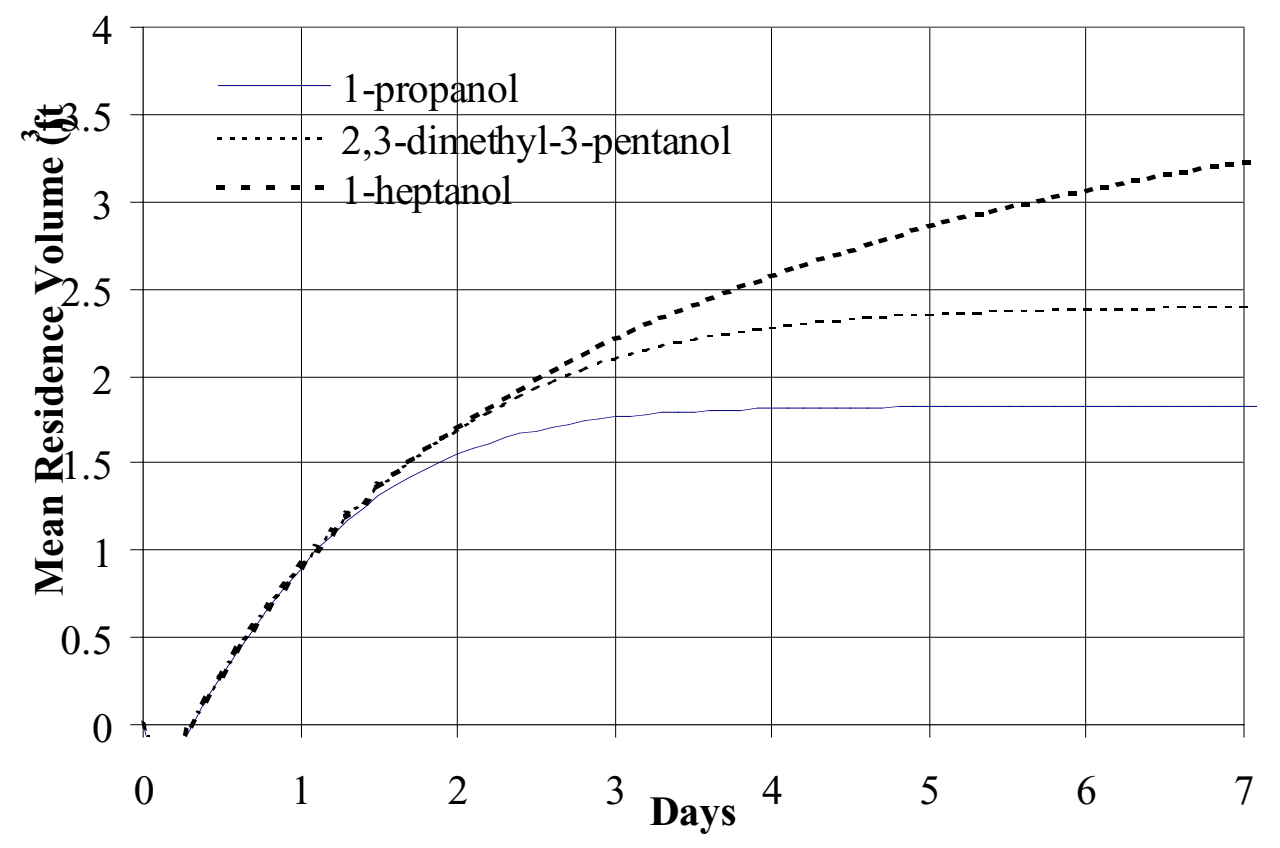

Figure 6.32: Cumulative tracer MRV for TT035.

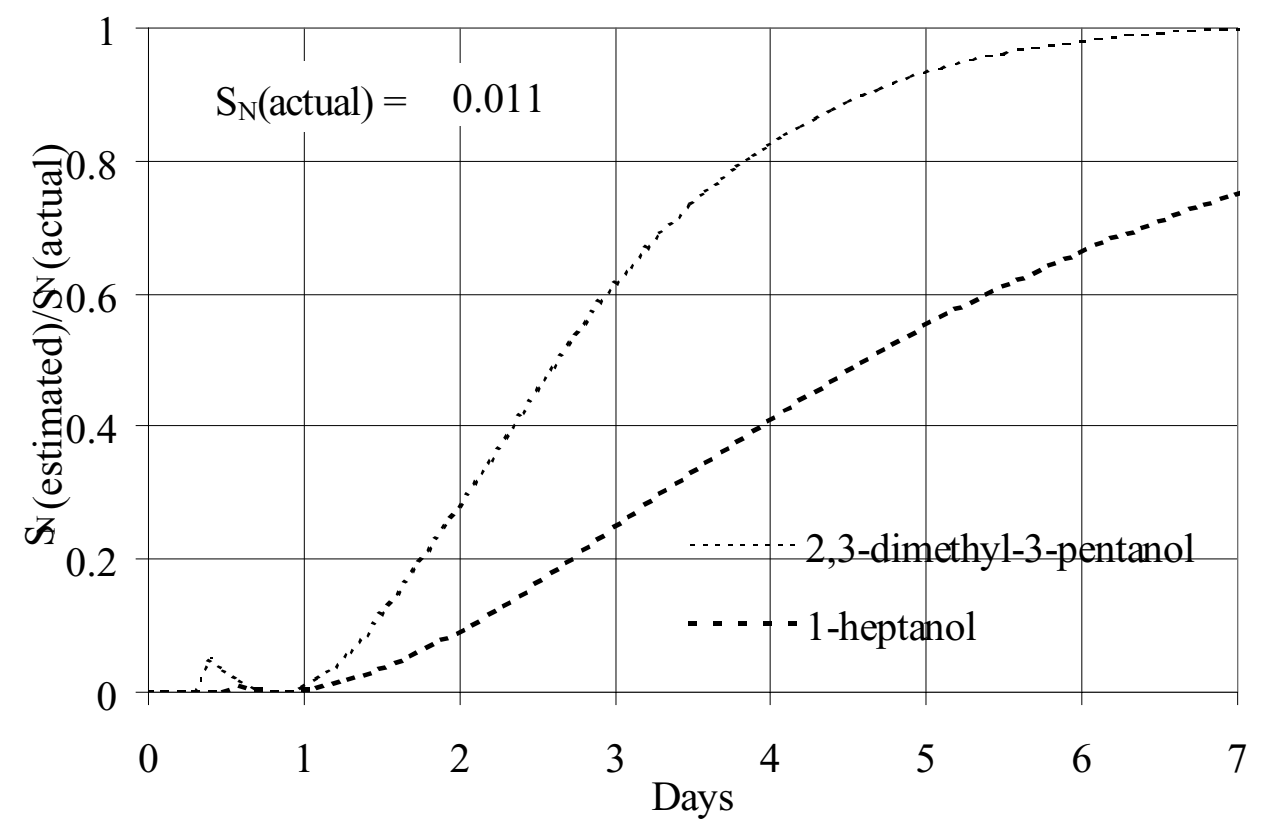

Figure 6.33: Cumulative estimated NAPL saturation for TT035. 


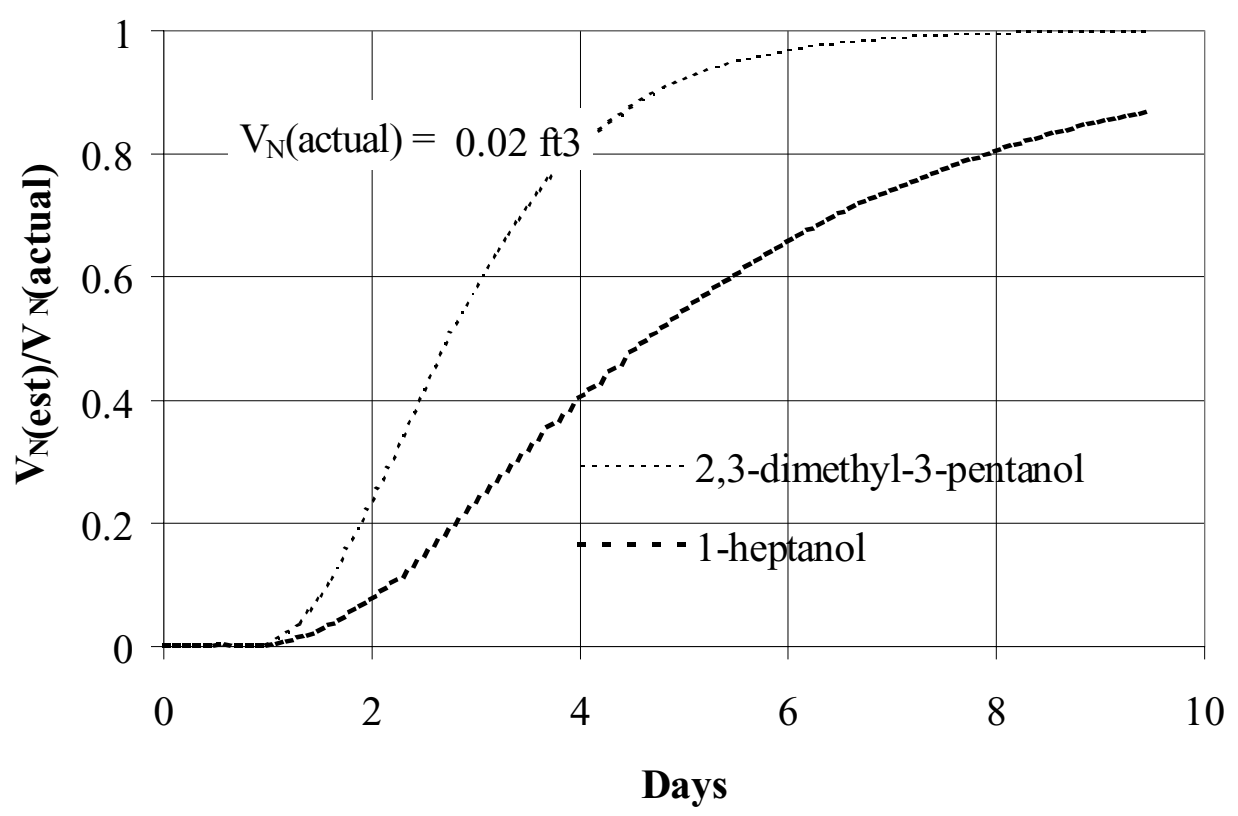

Figure 6.34: Cumulative estimated NAPL volume for TT035.

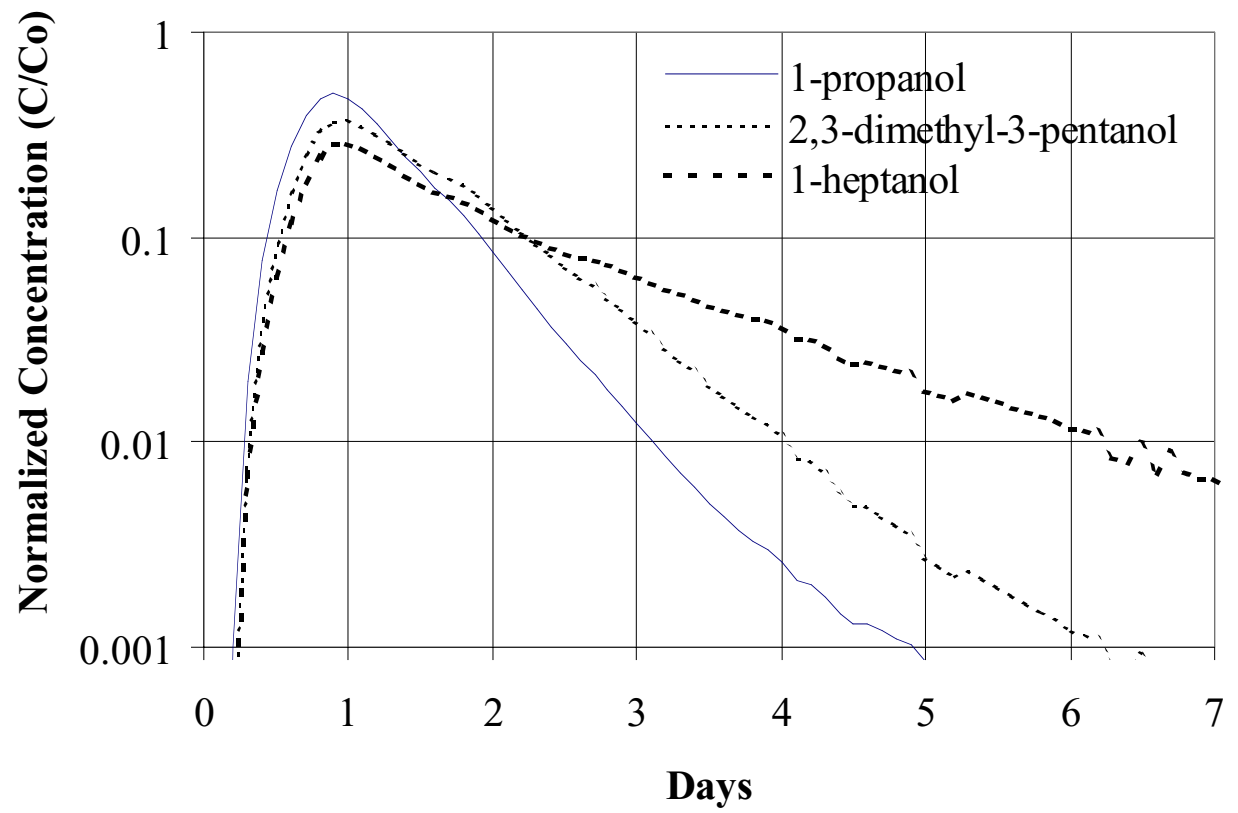

Figure 6.35: Tracer response curves for TT037. 


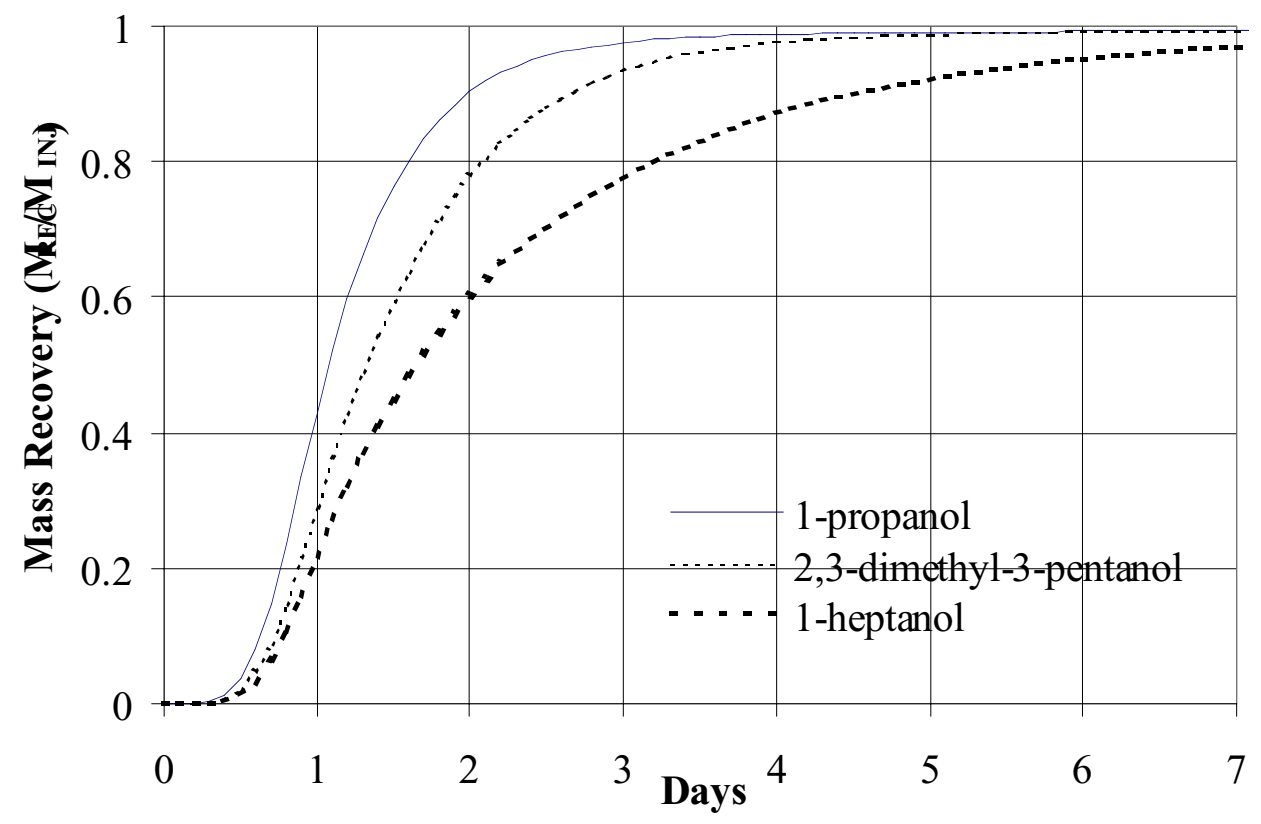

Figure 6.36: Cumulative tracer mass recovery for TT037.

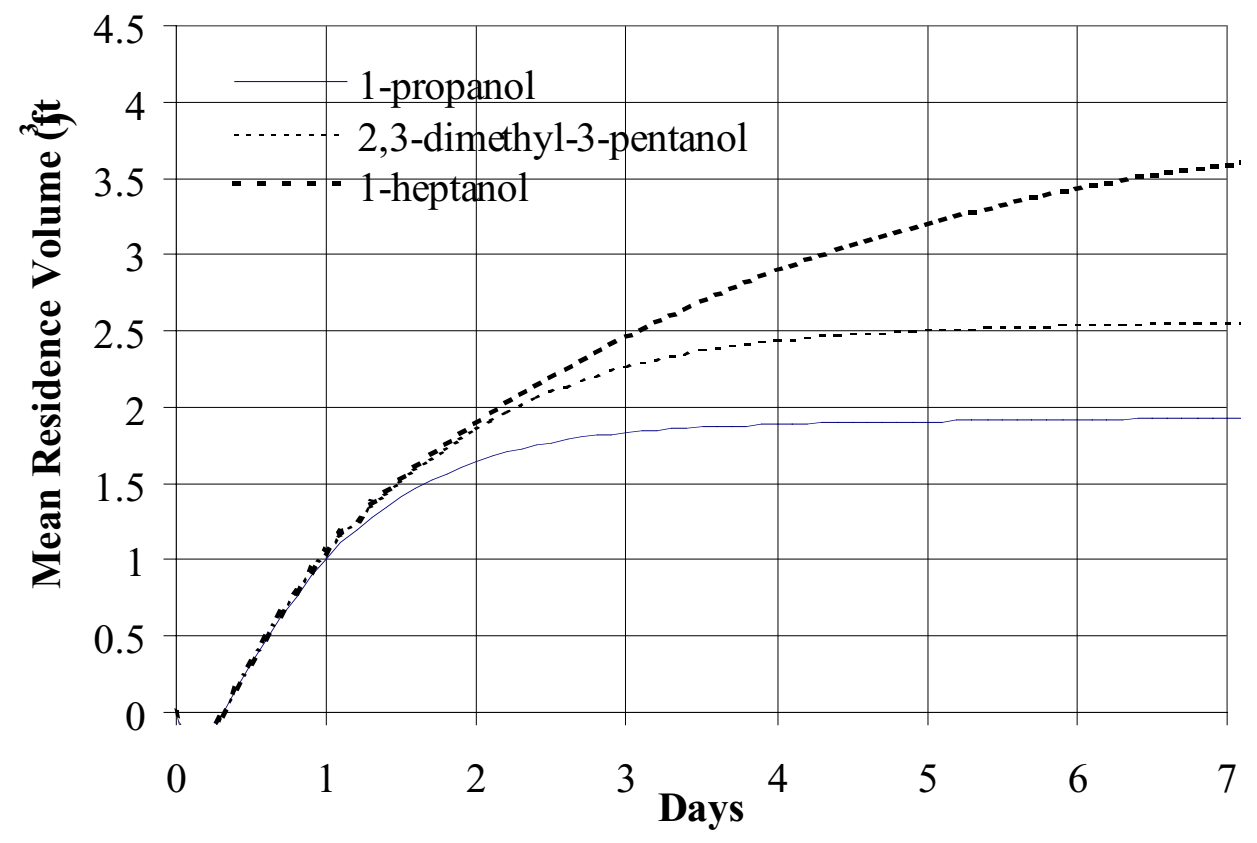

Figure 6.37: Cumulative tracer MRV for TT037. 


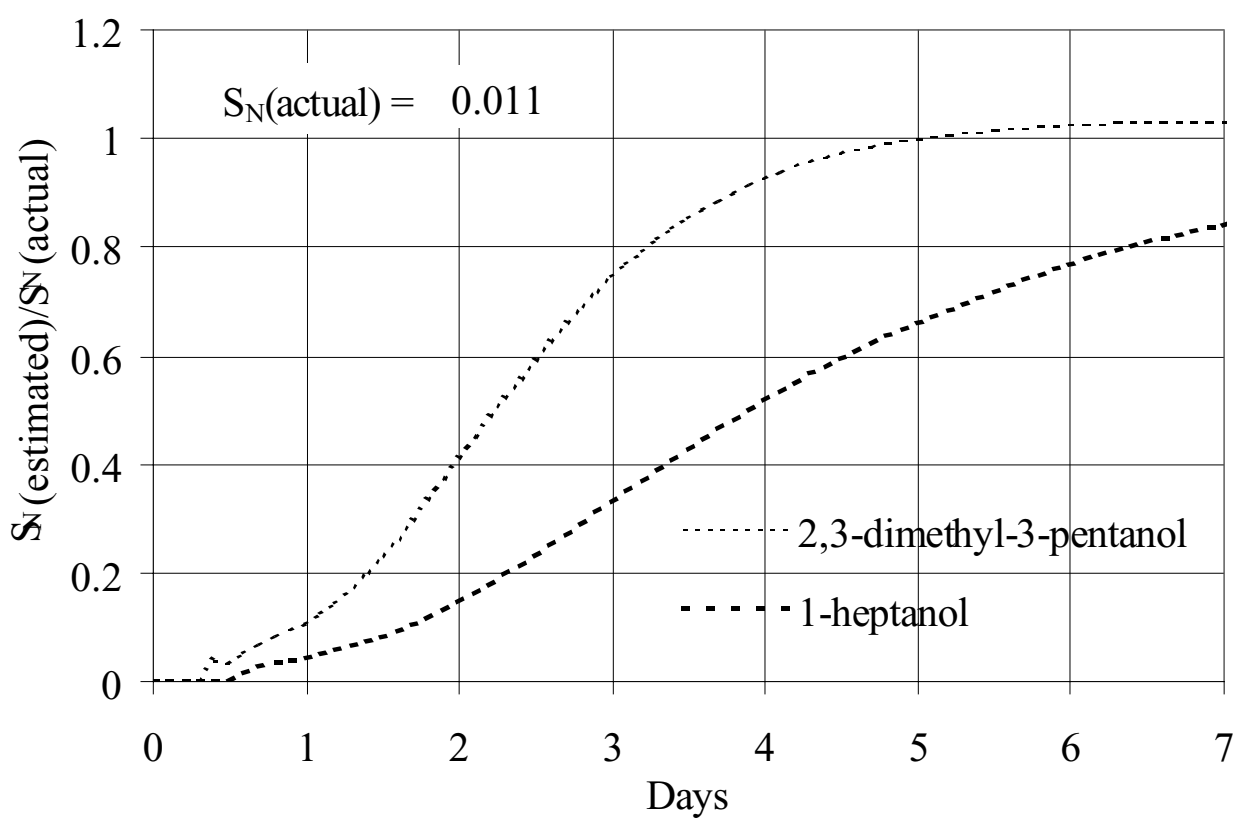

Figure 6.38: Cumulative estimated NAPL saturation for TT037.

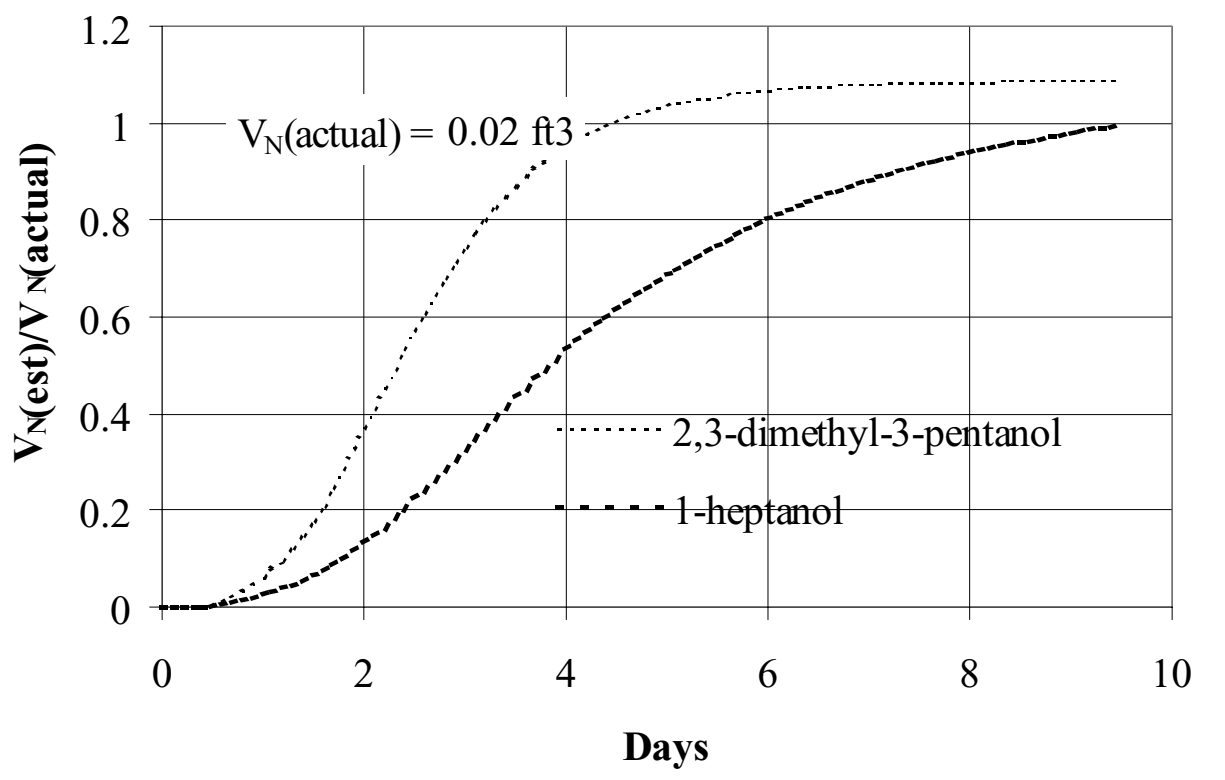

Figure 6.39: Cumulative estimated NAPL volume for TT037. 


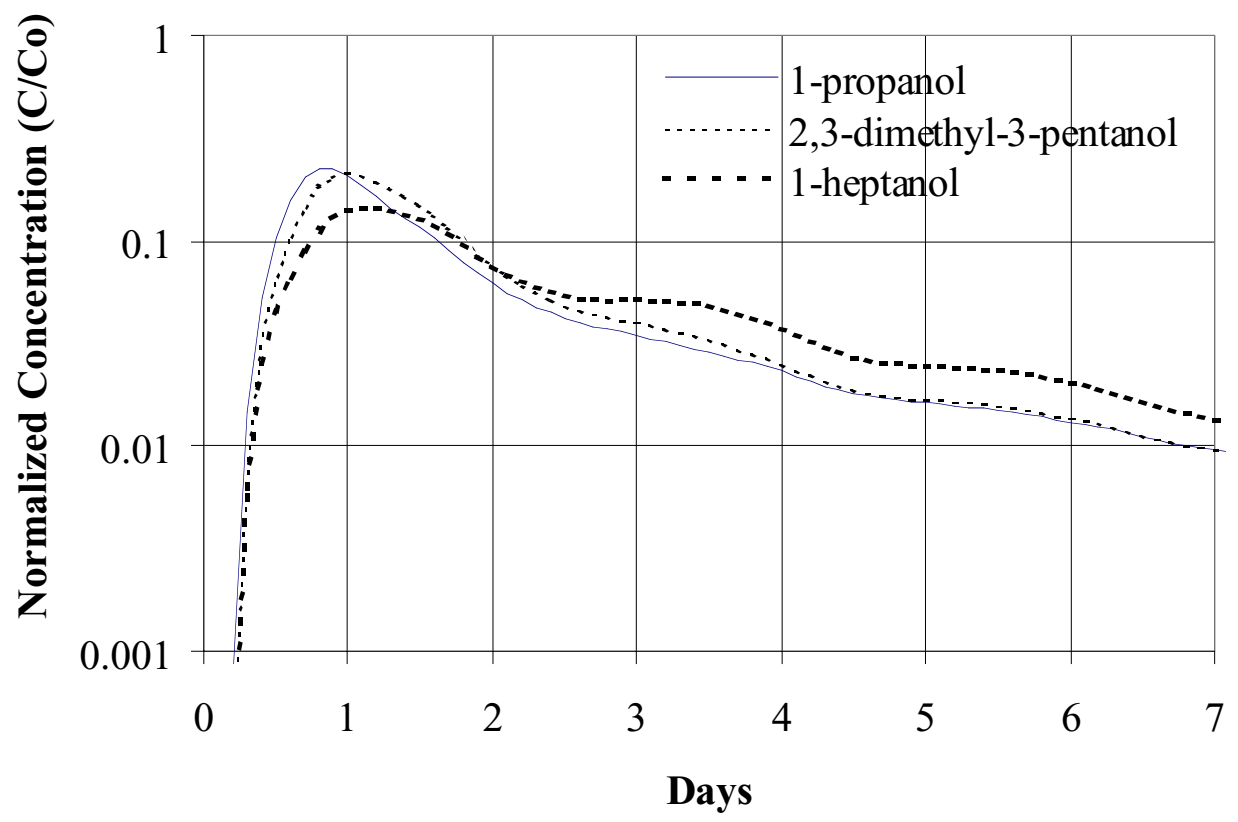

Figure 6.40: Tracer response curves for TT038.

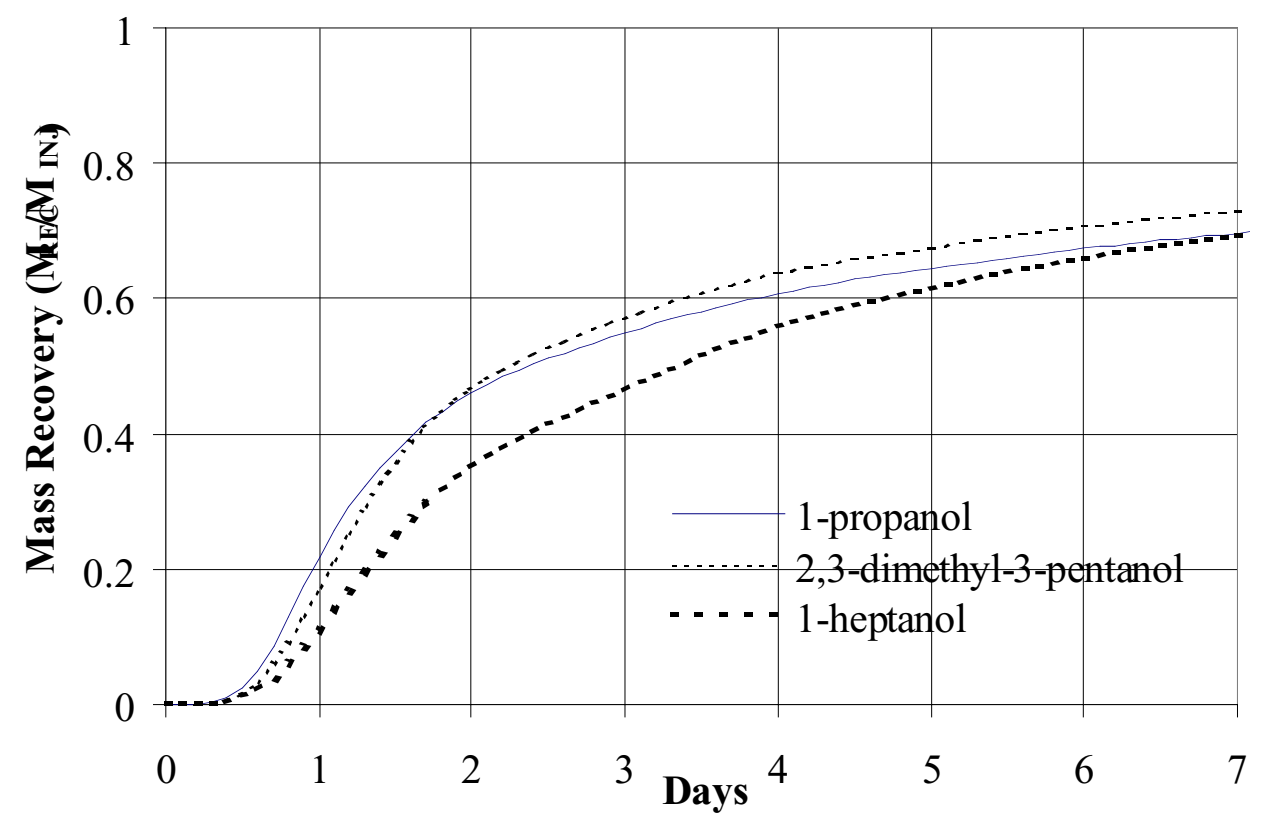

Figure 6.41: Cumulative tracer mass recovery for TT038. 


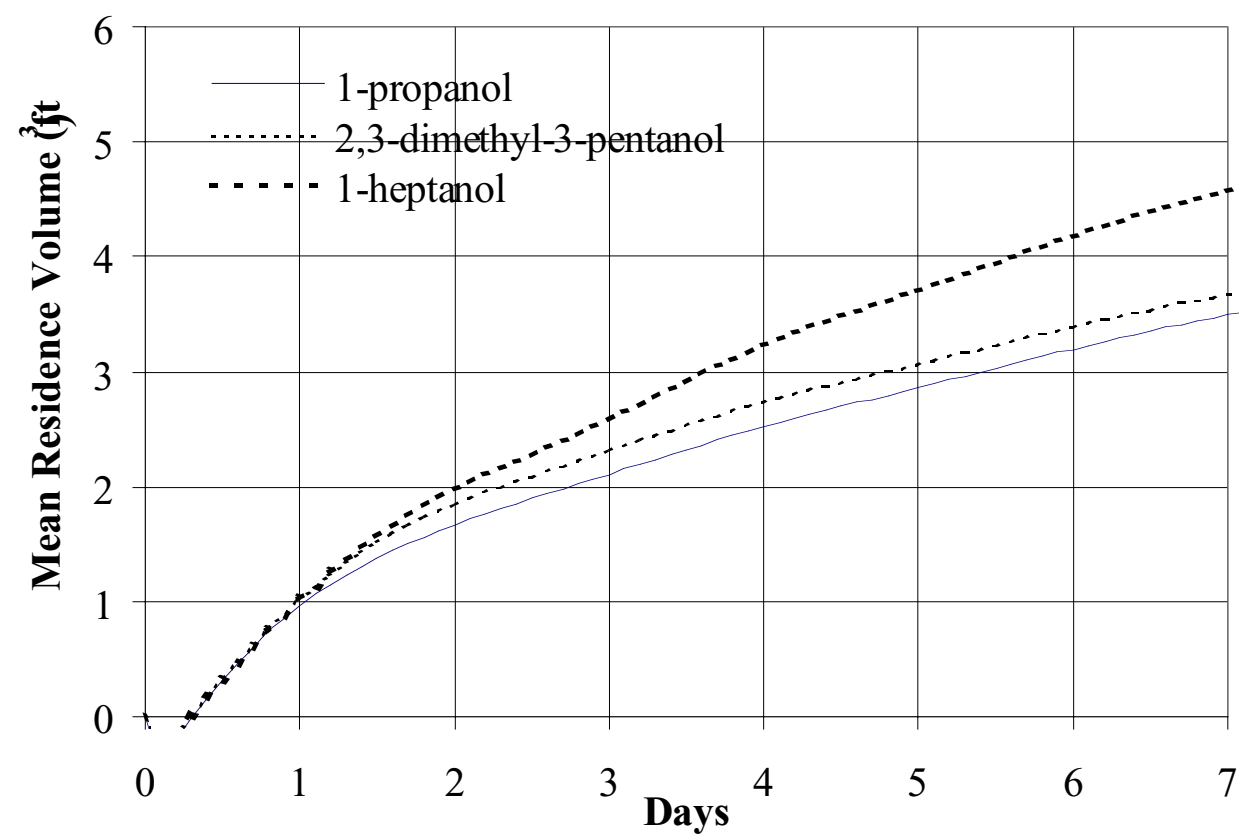

Figure 6.42: Cumulative tracer MRV for TT038.

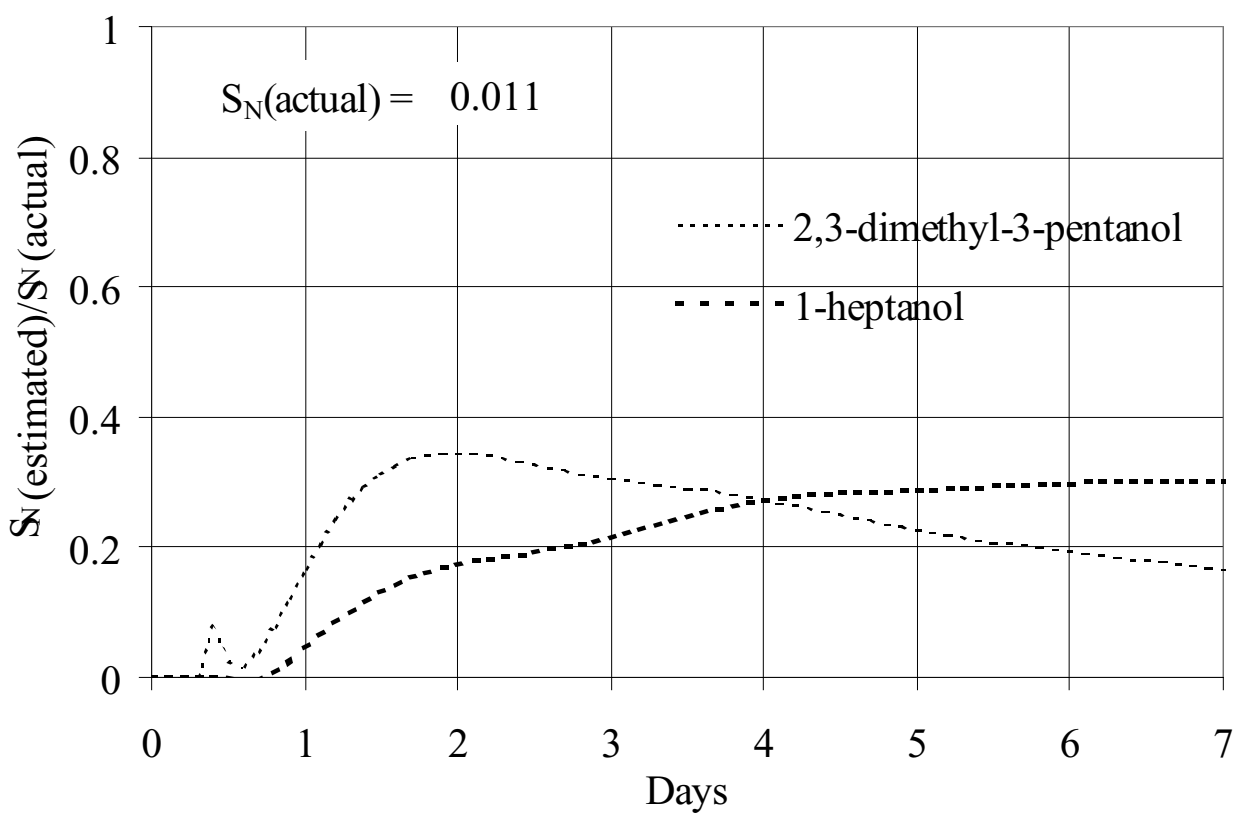

Figure 6.43: Cumulative estimated NAPL saturation for TT038. 


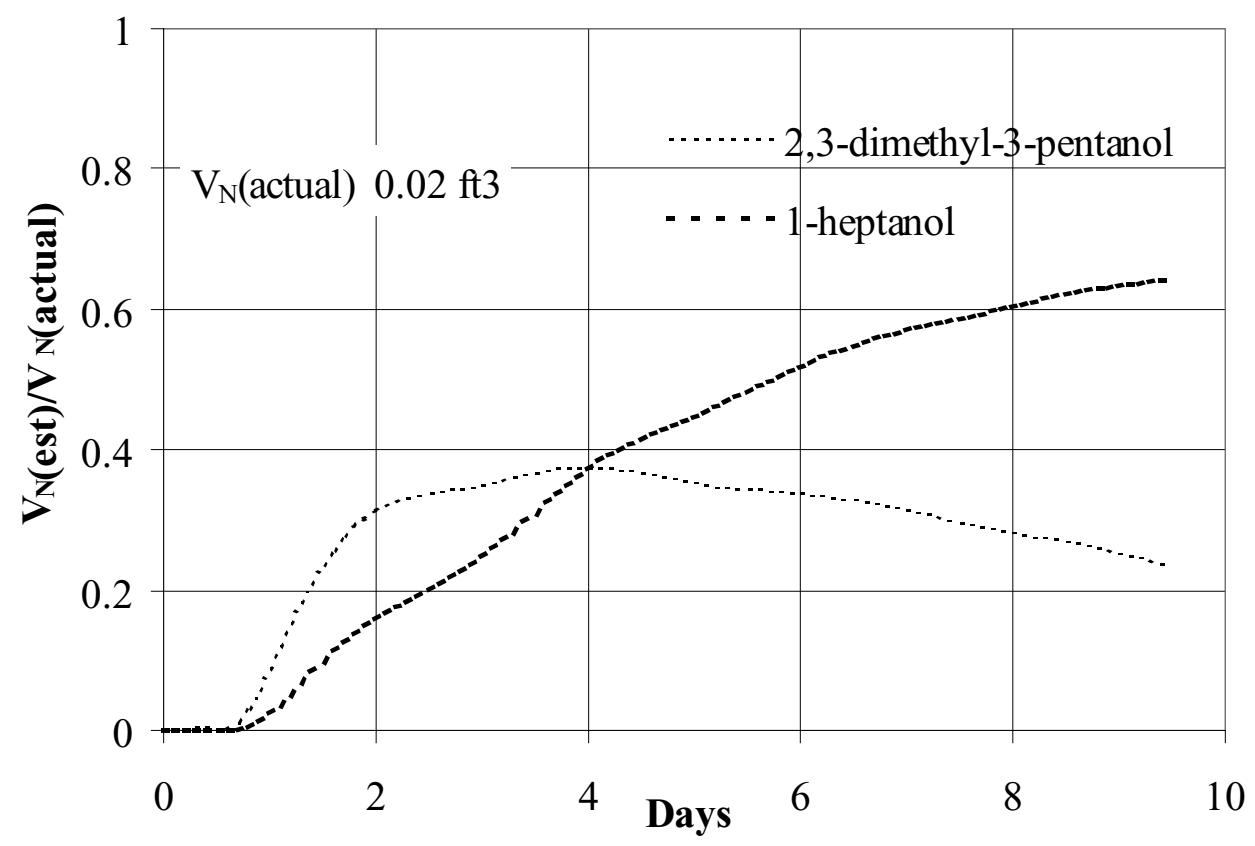

Figure 6.44: Cumulative estimated NAPL volume for TT038.

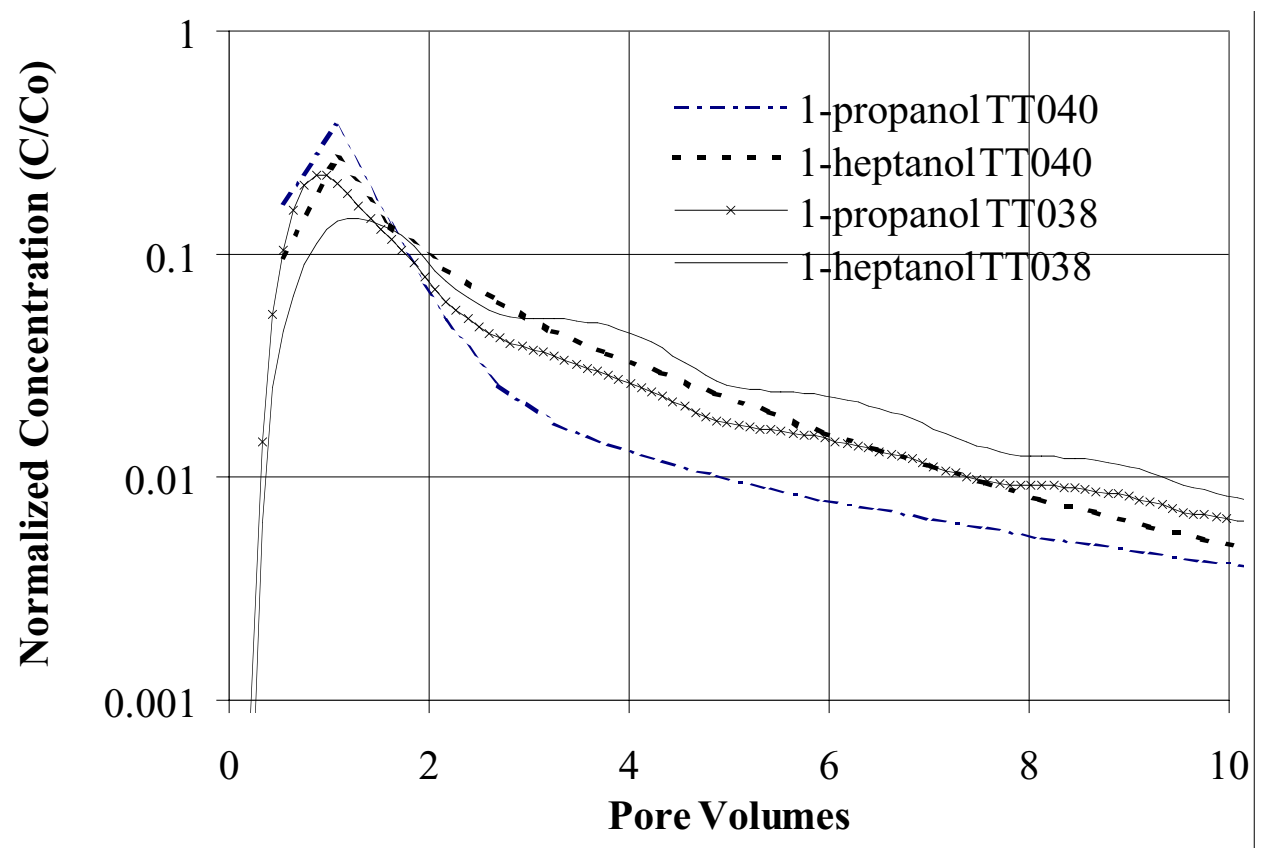

Figure 6.45: Tracer response curves comparing TT040 and TT038. 


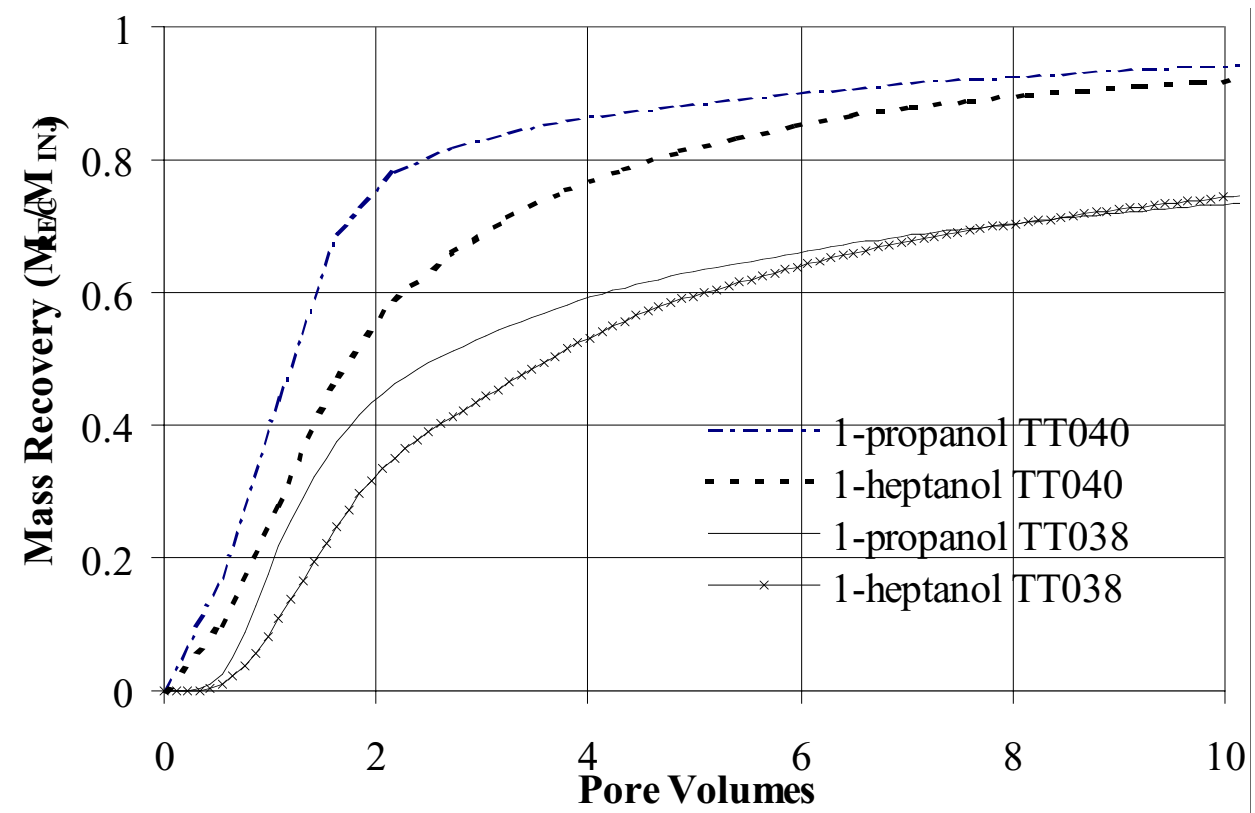

Figure 6.46: Cumulative mass recovery curves for TT040 and TT038.

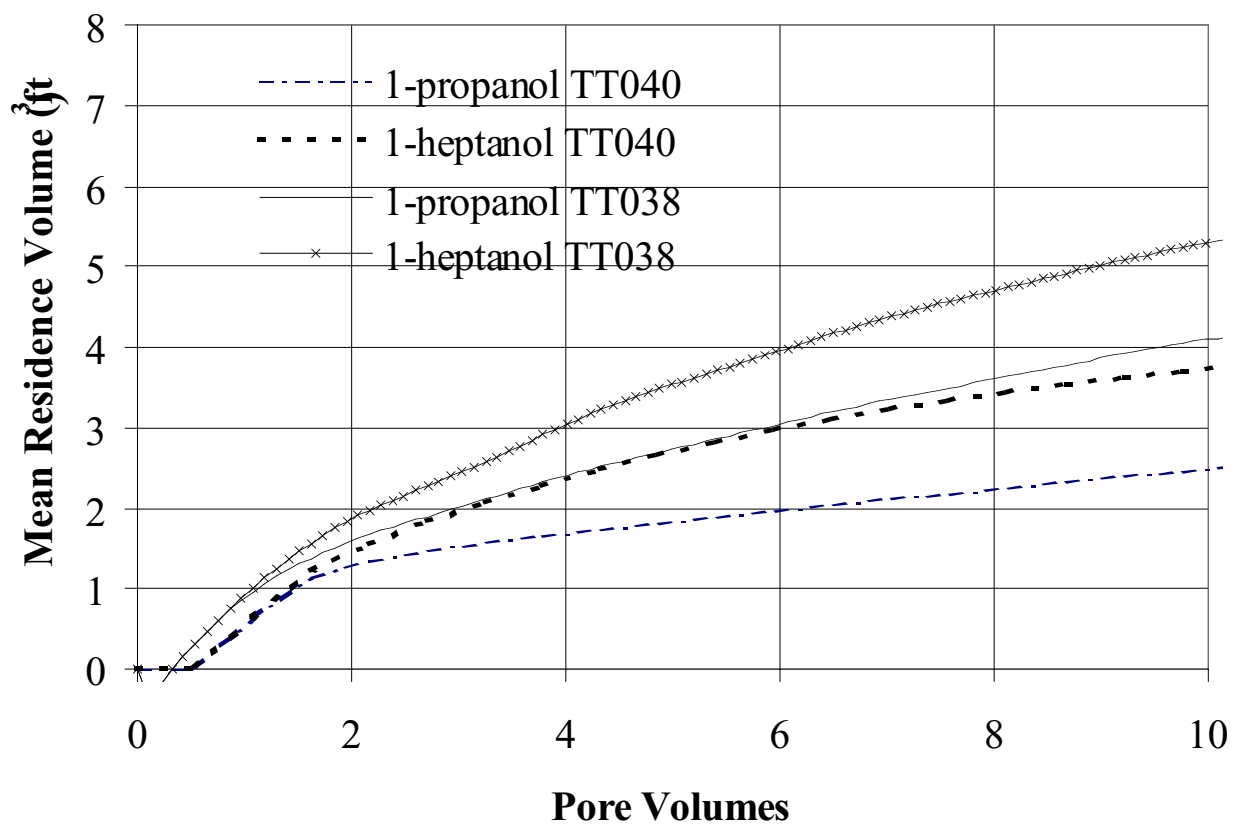

Figure 6.47: Cumulative mean residence volumes for TT040 and TT038. 


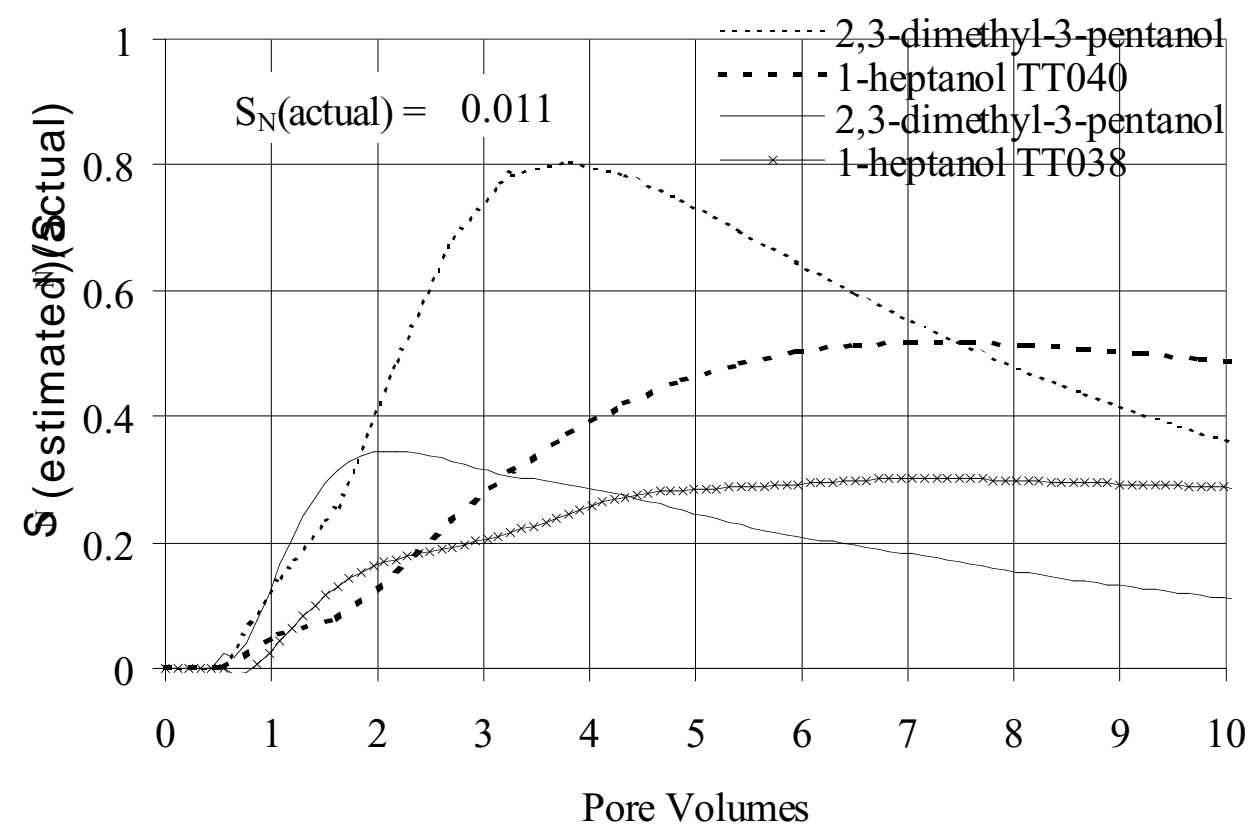

Figure 6.48: Cumulative estimated NAPL saturation for TT040 and TT038.

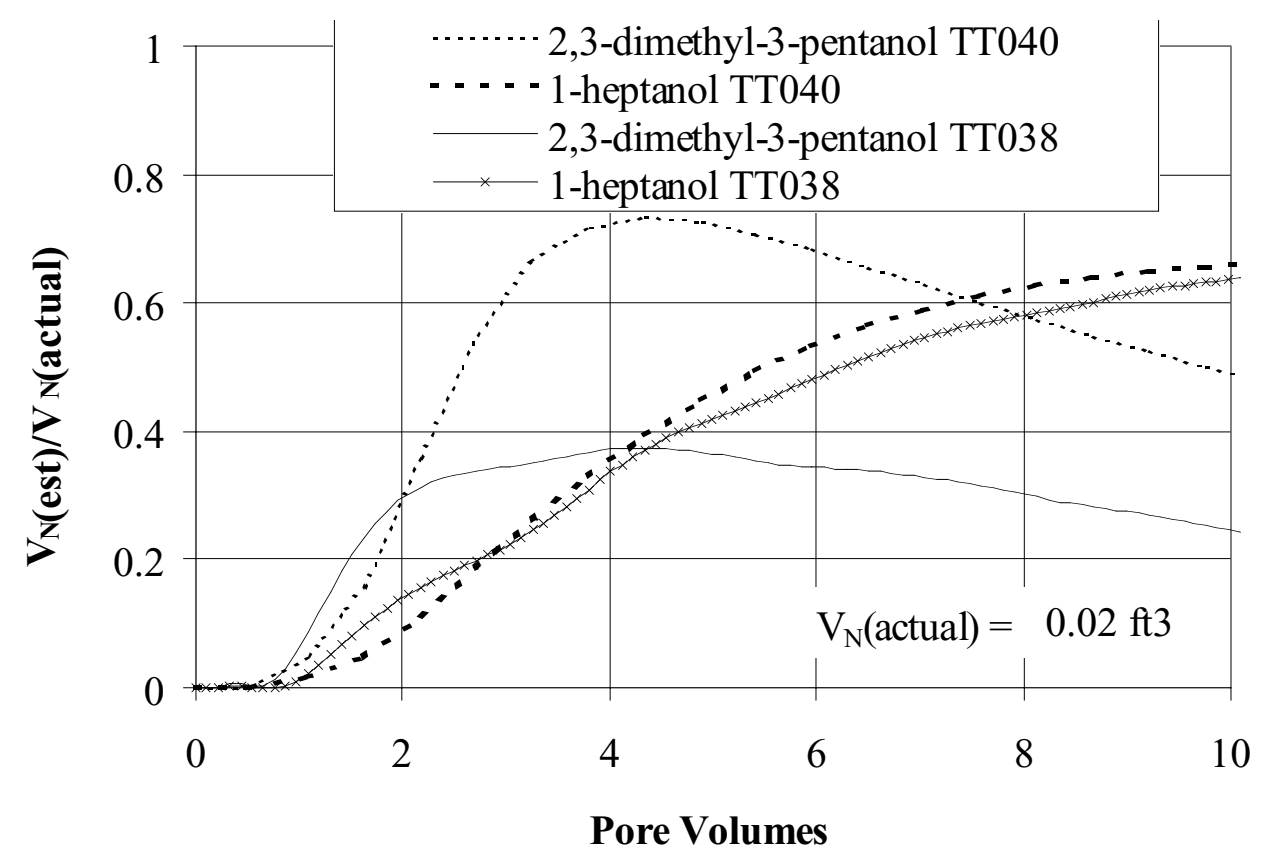

Figure 6.49: Cumulative estimated NAPL volume from TT040 and TT038. 


\section{Section 7: Summary, Conclusions and Recommendations for Future Work}

\subsection{Summary and Conclusions}

The objectives of this research were (1) to evaluate the potential of current PITT technology for characterizing NAPLs in fractured media and (2) to provide new experimental and theoretical research to help adapt the PITT technology for use in fractured media.

An aqueous phase mass transfer coefficient correlation was applied to tracers partitioning from water into NAPLs in porous media. A simple NAPL phase mass transfer coefficient was developed based on the assumption of spherical NAPL blobs in the porous media. A deterministic sensitivity analysis indicated that the NAPL phase resistance would be negligible for a range of input parameters typical for PITTs in porous media. The Damkohler number determined using this correlation was either independent of or very weakly dependent on the NAPL saturation, depending on the reported value of the saturation exponent.

A 1-D tracer transport model in porous media with linear, reversible mass transfer was derived. The solution in Laplace space was analyzed to determine the theoretical mean residence time of a partitioning tracer with mass transfer. The mean residence time was determined to be equal to the equilibrium case, given complete capture of the tracer response curve. The 1-D model was used to determine how mass transfer limitations affected the NAPL volume estimate given realistic time and measurement constraints. Mass transfer had a visual impact on the partitioning tracer response curves at Damkohler numbers of approximately 10 or less. Mass transfer had an impact on the NAPL volume estimate at Damkohler numbers of approximately 1 or less. 
The partitioning tracer mass transfer correlation was implemented in UTCHEM, at 3-D transport simulator. Field scale runs indicated that even at very small mean residence times, i.e., 0.1 day, mass transfer limitations did not effect the partitioning tracer concentration response curves. This result is dependent on the validity of the water phase mass transfer correlation, and on the assumption of negligible contribution by the NAPL phase resistance.

A 1-D parallel fracture model was derived that included partitioning tracer water/NAPL mass transfer. Dimensional analysis of the equations for this model resulted in a new parameter, termed the matrix number, that describes the ratio of theoretical fracture mean residence time to the time required to diffuse to the center of the matrix.

The zero and first moments were calculated for the model solution in Laplace space. The results indicated that the theoretical tracer mean residence time is independent of the matrix and Damkohler numbers. The fracture and matrix contributions to the mean residence time were separated from the overall mean residence time. If the tracer response curve is complete, the matrix contribution to the mean residence time dominates the overall mean residence time, for typical fractured media.

Sensitivity analyses with the model indicate variable accuracy in NAPL saturation estimates made from partitioning tracer data in fractured media, depending on the fracture porosity, matrix porosity, and matrix number. In general, high fracture porosities, low matrix porosities, and low matrix numbers all improve the PITT NAPL saturation estimate, because the overall mean residence time approaches the theoretical fracture mean residence time. Sensitivity analyses also indicated that using a conservative tracer with a higher diffusion coefficient than the partitioning tracers could significantly increase the error in the saturation estimate. 
Laboratory partitioning tracer experiments were completed in single fracture berea cores, both with and without NAPL present. The tracer response data in clean cores showed retardation and late time tailing. Results from an experiment with both inorganic and organic tracers of varying diffusion coefficients provided strong evidence that the retardation and late time tailing were caused by matrix diffusion. The higher the diffusion coefficient of the tracer, the more pronounced was the impact of matrix diffusion. A semi-analytical single fracture model was fit to the concentration response data in order to estimate some experimental parameters, such as average fracture aperture and average flow velocity. In general, the model fit the data well, with the worst fit occurring for the tracers with the lowest diffusion coefficients.

The model was used to simulate the tracer concentration in the matrix for one of the experiments. The simulation results showed that the tracer penetrated approximately 2 millimeters into the matrix, and that a maximum of $30 \%$ of the total tracer mass was in the matrix during the course of the experiment, much of which remained even after the fracture concentration approached zero.

Two experiments with varying flow rates verified the kinetic limitations that characterize matrix diffusion effects. The higher the average velocity of the tracer in the fracture, the less pronounced the effects of diffusion. Results from these experiments were also used to predict the dispersivity and dispersivity exponent for the fracture. The dispersivity exponent, at 2.5 , was higher than expected, but within the range of published values for single fracture experiments.

Two experiments were completed with NAPL in the fracture at two different flow rates. The partitioning tracer response curves showed both the effects of partitioning (retardation and lower peaks with respect to the conservative tracer) and matrix diffusion (more pronounced late time tailing of the lighter conservative tracer). Due to matrix diffusion, the mean residence 
volume of the conservative tracer increased faster than that of the partitioning tracers at late time, which caused a poor estimate of NAPL volume in the fracture compared to the measured value. An analysis of early time data extrapolation showed that the results from this technique were too sensitive to small errors in the estimated slope to yield good results.

A semi-analytical single fracture model fit the conservative tracer concentration data well, but fit the partitioning tracer concentration data poorly. The model was therefore not suitable for predicting concentration histories for partitioning tracers in NAPL saturated fractured cores. However, the model did provide a good fit for the tracer mean residence volume data. The NAPL volume calculated from the fit parameters for the higher flow rate experiment was closer to the measured value than the volume calculated from the lower flow rate experiment. The calculated volume for the higher flow rate experiment was still $38 \%$ lower than the measured value.

Fitting the semi-analytical model to the MRV tracer data was the most accurate method for estimating the NAPL volume in the fracture. Partitioning tracer tests in rocks of high tortuosity (where the diffusion coefficient in the rock is not several orders of magnitude lower than bulk diffusion coefficient in water), will not provide accurate estimates of resident NAPL volumes if interpreted with standard method of moment equations. Inverse modeling of the tracer response data with a simulator that accounts for matrix diffusion may provide a more accurate estimate.

Three dimensional DNAPL spill and PITT simulations were completed using UTCHEM with the dual-porosity option enabled. First, the matrix block subgridding in UTCHEM was analyzed to determine the optimum number and placement of subgrids for given simulation parameters. In general, approximately 8 subgrids are required in both directions concentrated in 
the first 1.5 standard deviations of tracer concentration, calculated using the estimated total PITT duration.

The capillary pressure parameters for the spill were determined from existing experimental data. The DNAPL spill resulted in high trapped saturations (i.e., 0.65) in some zones with very low saturations in other zones. The subsequent waterflood induced a more uniform distribution of the DNAPL with most of the saturations ranging from 0.01 to 0.15 .

The PITTs gave a good estimate of the average DNAPL saturation and DNAPL volume at low matrix porosity and correspondingly low (i.e., 0.01) matrix tortuosity. As the porosity and tortuosity were increased, the DNAPL saturation and volume estimates degraded, with the smaller (more diffusive) partitioning tracer response affected more significantly than the heavier partitioning tracer response. The NAPL volume estimate was shown to be more accurate than the NAPL saturation estimate for typical fractured media conditions.

Increasing the flow rate for the PITT increased the accuracy of both the NAPL saturation and volume estimates. The increased flow rate improved the result for the lighter, more diffusive partitioning tracer more significantly than for the heavier partitioning tracer. In general, the flow rate should be increased as much as possible without causing non-equilibrium effects with respect to the partitioning of the tracers between the NAPL and water. Because no mass transfer correlation exists for partitioning tracers in fractured media, direct determination of a nonequilibrium rate threshold was not possible.

\subsection{PITT Design in Fractured Media}

The findings in this research point to several key factors that should be considered when designing a PITT for fractured media: 
1. Consider the matrix rock type. The tortuosity of the rock matrix can vary several orders of magnitude from a high porosity rock such as Berea sandstone to a low porosity rock such as granite. Increasing tortuosity increases matrix diffusion effects, which in turn increase the error in the NAPL volume estimate.

2. Use tracers with similar molecular weights. Using a non-partitioning tracer that has a significantly higher diffusion coefficient than the partitioning tracers will increase the error in the NAPL volume estimate. In general, a lighter conservative tracer gives results that underestimate the NAPL volume.

3. Design for the highest possible flow rate where local equilibrium can still be achieved. Increasing the average velocity of the mobile phase will decrease the impact of matrix diffusion, improving the NAPL volume estimate. Note that the upper limit for the flow rate is set by the minimum mean residence time required for equilibrium between the partitioning tracers and the NAPL. Experiments are necessary to determine the mean residence time required to achieve this local equilibrium.

4. Analyze the data with a model that accounts for matrix diffusion. Accounting for matrix diffusion during the tracer transport improves the NAPL volume estimate, by helping to separate matrix diffusion effects from partitioning effects.

\subsection{Recommendations for Future Work}

1. Expand the current research to the case where the NAPL resides in the rock matrix.

2. Complete laboratory studies of water-NAPL partitioning tracer mass transfer in porous media to measure the relative contribution of NAPL phase mass transfer resistance.

3. Complete laboratory studies of water-NAPL mass partitioning tracer mass transfer in fractured media. 
4. Model additional scenarios at the field scale to increase the available sensitivity data.

5. Perform an actual field scale PITT in fractured media. 



\section{References}

Annable, M. D., P. S. C. Rao, K. Hatfield, W. D. Graham and A. L. Wood: "Use of Partitioning Tracers for Measuring Residual NAPL Distribution in a Contaminated Aquifer: Preliminary Results From a Field-Scale Test," Proc., 2nd Annual Tracer Workshop, Univ. of Texas at Austin, November 14-15, 1994.

Annable, M.D., P. S. C. Rao, K. Hatfield, W. D. Graham, A. L. Wood and C. G. Enfield: "Partitioning Tracers for Measuring Residual NAPL: Field-Scale Test Results," $J$. Environ. Eng., 124(6), 498-503, 1998.

Bear, J.: Dynamics of Fluids in Porous Media, American Elsevier Pub. Co., New York, 1972.

Becker, M. W.: "Tracer Studies in a Fractured Rock and Their First Passage Time Mathematical Models," Dissertation, University of Texas at Austin, 1996.

Borchers, B., T. Uram and J. M. H. Hendrickx: "Tikhonov Regularization of Electrical Conductivity Depth Profiles in Field Soils," Soil Sci. Soc. Am. J., 61, 1004-1009, 1997.

Brown, C. L., M. Delshad, V. Dwarakanath, D. C. McKinney and G. A. Pope: "Design of a Field-Scale Surfactant Enhanced Remediation of a DNAPL Contaminated Aquifer," Presented at the I\&EC Special Symposium American Chemical Society Birmingham, AL, September 9-12, 1996.

Brown, C. L., M. Delshad, V. Dwarakanath, R. E. Jackson, J. T. Londergan, H. W. Meinardus, D. C. McKinney, T. Oolman, G. A. Pope and W. H. Wade: "Demonstration of Surfactant Flooding of an Alluvial Aquifer Contaminated with DNAPL," in Innovative Subsurface Remediation: Field Testing of Physical, Chemical, and Characterization Technologies, ACS Symposium Series 725, 1999.

Constable, S. C., R. L. Parker and C. G. Constable: "Occam's Inversion: A Practical Algorithm for Generating Smooth Models From Electromagnetic Sounding Data," Geophysics, 52(3), 289-300, 1987.

Datta-Gupta, A. and M. J. King: "A Semianalytical Approach to Tracer Flow Modeling in Heterogeneous Permeable Media," Advances in Water Resources, 18, 9-24, 1995.

Deeds, N. E., G. A. Pope and D. C. McKinney: "Vadose Zone Characterization at a Contaminated Field Site Using Partitioning Interwell Tracer Technology, Environmental Science and Technology, in press, September, 1999.

Delshad, M., G. A. Pope and K. Sepehrnoori: "A Compositional Simulator for Modeling Enhanced Aquifer Remediation," formulation, Journal of Contaminant Hydrology, 23, 303-327, 1996.

Donaldson, E. C., R. F. Kendall and F. S. Manning: "Dispersion and Tortuosity in Sandstones," 51st Annual Fall Technical Conference and Exhibition of the Society of Petroleum Engineers of AIME, held in New Orleans, Oct. 3-6, SPE 6190, 1976.

Dronfield, D. G. and S. E. Silliman: "Velocity Dependence of Dispersion for Transport Through a Single Fracture of Variable Roughness," Water Resour. Res, 29(10), 3477-3483, October 1993. 
Dwarakanath, V.: "Characterization and Remediation of Aquifers Contaminated by Non-aqueous Phase Liquids Using Partitioning Tracers and Surfactants," Dissertation, The University of Texas, Austin, 1997.

Dwarakanath, V. and G. A. Pope: "A New Method for Estimating Alcohol Partition Coefficients Between Non-aqueous Phase Liquids and Water," Environmental Science and Technology, 32, 1662, 1998.

Edgar, J. R.: "Laboratory Evaluation of Partitioning Tracers, Thesis, University of Texas at Austin, December 1997.

EPA: "Three-Dimensional NAPL Fate and Transport Model," EPA/600/R-99/011, Office of Research and Development, February 1999.

Fetter, C.W.: Contaminant Hydrogeology, Macmillian Publishing Company, New York, NY, 1993.

Gelhar, L. W. and M. A. Collins: "General Analysis of Longitudinal Dispersion in Nonuniform Flow," Water Resour. Res., 7, 1511-1521, 1971.

Golub, G. H. and C. F. Van Loan: Matrix Computations, Johns Hopkins University Press, Baltimore, 1989.

Harvey, C. F. and S. M. Gorelick: "Temporal Moment-Generating Equations: Modeling Transport and Mass Transfer in Heterogeneous Aquifers," Water Resources Research, 31(8), 1895-1911, 1995.

Imhoff, P. T., A. Frizzell and C. T. Miller: Environmental Science and Technology, 31, 1997, 1615-1622.

Imhoff, P. T., M. H. Arthur and C. T. Miller: "Complete Dissolution of Trichloroethylene in Saturated Porous Media," Environmental Science and Technology, 32, 1998, 2417-2424.

Imhoff, P. T., P. R. Jaffe and G. F. Pinder: "An Experimental Study of Complete Dissolution of a Non-Aqueous Phase Liquid in Saturated Porous Media," Water Resources Research, 30, 2, 1994, 307-320.

Jackson, R. E. and V. Dwarakanath: "The Physical Chemistry of Chlorinated Degreasing Solvents: A Critical Review of those Properties and Proceses Affecting Aquifer Contamination and Remediation," Ground Water, in press, 1999.

James, A. I., W. D. Graham, K. Hatfield, P. S. C. Rao and M. D. Annable: "Optimal Estimation of Residual Non-Aqueous Phase Liquid Saturations Using Partitioning Tracer Concentration Data," Water Resour. Res., 33(12), 2621-2636, 1997.

Jennings, Jr., J. W., S. C. Ruppel and W. B. Ward: "Geostatistical Analysis of Petrophysical Data and Modeling of Fluid-Flow Effects in Carbonate Outcrops," Society of Petroleum Engineers, SPE 49025, 1998.

Jin, M., M. Delshad, V. Dwarakanath, D. C. McKinney, G. A. Pope, K. Sepehrnoori, C. E. Tilburg and R. E. Jackson: "Partitioning Tracer Test for Detection, Estimation, and Remediation Performance Assessment of Subsurface Non Aqueous Phase Liquids," Water Resour. Res., 31(5), 1201-1211, 1995. 
Kazemi, H. and J. R. Gilman,: "Multiphase Flow in Fractured Petroleum Reservoirs," in Flow and Contaminant Transport in Fractured Rock, Academic Press Inc., London, 1993.

King, M. J. and A. Datta-Gupta: "Streamline Simulation: A Current Perspective," In Situ, 22(1), 91-140, 1998.

Kueper, B. H. and D. B. McWhorter: "The Behavior of Dense, Non-Aqueous Phase Liquids in Fractured Clay and Rock," Ground Water, 29, 716-728, 1991.

Liu, C. and W. P. Ball: "Application of Inverse Methods to Contaminant Source Identification From Aquitard Diffusion Profiles at Dover AFB, Delaware," Water Resour. Res., 35(7), 1975-1985, 1999.

Londergan, J. T., H.W. Meinardus, P. E. Mariner and R. E. Jackson: "DNAPL Removal From a Heterogeneous Alluvial Aquifer by Surfactant-Enhanced Aquifer Remediation," Ground Water, in press, 1999.

Maloszewski, P. and A. Zuber: "Mathematical Modeling of Tracer Behavior in Short-Term Experiments in Fissured Rocks," Water Resourc. Res., 26(7) 1517-1528, 1990.

Maloszewski, P., and A. Zuber, Tracer experiments in fracture rocks: matrix diffusion and the validity of models, Water Resour. Res . 29(8), 2723-2735, 1993.

McLaughlin, D. and L. R. Townley: "A Reassessment of the Groundwater Inverse Problem," Water Resour. Res., 32(5), 1131-1161, 1996.

Menke, W.: Geophysical Data Analysis: Discrete Inverse Theory, Academic Press, San Diego, 1989.

Miller, C. T., M. M. Poirier-McNeill and A. S. Mayer: "Dissolution of Trapped Nonaqueous Phase Liquids: Mass Transfer Characteristics," Water Resources Research, 26, 11, 1990, 2783-2796.

Neretnieks, I.: "A Note on Fracture Flow Dispersion Mechanisms in the Ground," Water Resour. Res., 19(2), 364-370, 1983.

Neretnieks, I.: "Solute Transport in Fractured Rock: Applications to Radionuclide Waste Repositories," in Flow and Contaminant Transport in Fractured Rock, Academic Press Inc., London, 1993.

Paige, C. C. and M. A. Saunders: "LSQR: An Algorithm for Sparse Linear Equations and Sparse Least Squares," ACM Transactions on Mathematical Software, 8(1), 43-71, 1982.

Powers, S. E., L. M. Abriola and W. Weber: "An Experimental Investigation of Nonaqueous Phase Liquid Dissolution in Saturated Subsurface Systems: Transient Mass Transfer Rates," Water Resources Research, 30, 2, 1994, 321-332.

Reid, R. C., J. M. Prausnitz and B. E. Poling: The Properties of Gases \& Liquids, McGraw-Hill, Inc., New York, NY, 1987.

Reitsma, S. and B. H. Kueper: "Laboratory Measurement of Capillary Pressure-Saturation Relationships in a Rock Fracture," Water Resources Research, 30, 4, 1994, 865-878.

Robinson, B. A. and J. W. Tester: "Dispersed Fluid Flow in Fractured Reservoirs: An Analysis of Tracer-Determined Residence Time Distributions," J. of Geophysical Res., 89(B12), 10374-10384, 1984. 
Sahimi, M.: Flow and Contaminant Transport in Porous Media and Fractured Rock, VCH: Weinheim (Federal Republic of Germany), cop. 1995.

Seader, J. D. and E. J. Henley: Separation Process Principles, John Wiley and Sons, New York, 1998.

Snow, D. T.: "The Frequency and Apertures of Fractures in Rock," Journal of Rock Mechanics and Mining Sciences, 7, 23-40, 1970.

Sun, N-Z. and W. W-G. Yeh: Coupled Inverse Problems in Groundwater Modeling,1. Sensitivity Analysis and Parameter Identification," Water Resour. Res., 26, 2507-2525, 1990.

Tang, D. H., E. O. Frind and E. A. Sudicky: "Contaminant Transport in Fractured Porous Media: Analytical Solution for a Single Fracture," Water Resour. Res., 17 (3), 555-564, 1981.

Treybal, R. E.: Liquid Extraction, McGraw-Hill Book Company, New York, 1963.

Vasco, D. W. and A. Datta-Gupta: "Asymptotic Solutions for Solute Transport: A Formalism for Tracer Tomography," Water Resour. Res., 35(1), 1-16, 1999.

Vasco, D. W., S. Yoon and A. Datta-Gupta: "Integrating Dynamic Data into High-Resolution Reservoir Models Using Streamline-Based Analytic Sensitivity Coefficients," to be published in Dec. SPE Journal, 1999.

Vermuelen, T.: "Separation by Adsorption Methods," in Advances in Chemical Engineering, McGraw-Hill, New York, 147-209, 1958.

Whitley, G. A.: "An Investigation of Partitioning Tracer for Characterization of Nonaqueous Phase Liquids In the Vadose Zones," Dissertation, University of Texas at Austin, 1997.

Whitman, W. G.: Chem. and Met. Eng. 29, 147, 1923.

Wilson, R. D. and D. M. Mackay: "Direct Detection of Residual Nonaqueous Phase Liquid in the Saturated Zone Using $\mathrm{SF}_{6}$ as a Partitioning Tracer," Environmental Science \& Technology, 29(5), 1255-1258, 1995.

Yaws, C. L.: Handbook of Transport Property Data: Viscosity, Thermal Conductivity, and Diffusion Coefficients of Liquids and Gases, Gulf Publishing Company, Houston, 1995.

Yeh, W. W-G.: "Review of Parameter Identification Procedures in Groundwater Hydrology: The Inverse Problem," Water Resour. Res., 22(2), 95, 1986.

Yoon, S.: "Production data integration techniques for high-resolution reservoir description using streamline models," Dissertation, Texas A\&M University. (in progress).

Zhou, C., W. Cai, Y. Luo, G. T. Schuster and S. Hassanzadeh: "Acoustic Wave-Equation Traveltime and Waveform Inversion of Crosshole Seismic Data," Geophysics, 60(3), 765-773, 1995. 